\title{
EXAMINING ASSOCIATIONS BETWEEN INTERNALIZING BEHAVIOUR, CORTISOL REACTIVITY, AND PHYSIOLOGICAL COORDINATION IN TWO DIFFERENTIALLY POTENT CHALLENGES
}

\author{
by \\ Jennifer Khoury \\ Bachelor of Science, University of Toronto, 2011 \\ Master of Arts, Ryerson University, 2013
}

\begin{abstract}
A dissertation presented to Ryerson University in partial fulfillment of the requirements for the degree of Doctor of Philosophy in the Program of Psychology
\end{abstract}

Toronto, Ontario, Canada, 2017

(C) Jennifer Khoury 2017 


\section{AUTHOR'S DECLARATION FOR ELECTRONIC SUBMISSION OF A DISSERTATION}

I hereby declare that I am the sole author of this dissertation.

This is a true copy of the dissertation, including any required final revisions, as accepted by my examiners.

I authorize Ryerson University to lend this dissertation to other institutions or individuals for the purpose of scholarly research.

I further authorize Ryerson University to reproduce this dissertation by photocopying or by other means, in total or in part, at the request of other institutions or individuals for the purpose of scholarly research.

I understand that my dissertation may be made electronically available to the public. 


\begin{abstract}
EXAMINING ASSOCIATIONS BETWEEN INTERNALIZING BEHAVIOUR, CORTISOL REACTIVITY, AND PHYSIOLOGICAL COORDINATION, IN TWO DIFFERENTIALLY POTENT CHALLENGES
\end{abstract}

\author{
Doctor of philosophy, 2017 \\ Jennifer Khoury \\ Psychology, Ryerson University
}

Background: Research examining associations between child internalizing behaviours and cortisol reactivity is equivocal, with studies suggesting positive, negative and non-significant associations. The present study assessed three primary confounds that contribute to these inconsistencies: 1) the differential effectiveness of laboratory challenges in eliciting cortisol reactivity; 2) the impact of coordination between the Hypothalamic-Pituitary-Adrenal axis and the Sympathetic-Adrenal-Medullary system (assessed via salivary alpha-amylase (sAA)); and 3) variation in the measurement of internalizing behaviour, specifically, parent versus child ratings. The primary aims of this study were to assess internalizing behaviours in relation to both cortisol reactivity and coordination between cortisol and sAA, measured in two distinct challenges.

Method: A community sample of 8-10 year olds $(\mathrm{N}=52)$ participated in two laboratory challenges, across two study sessions: 1) the Trier Social Stress Test-Child Version (TSST-C), a potent social-evaluative challenge, and 2) a less-potent competition challenge, composed of a puzzle and mirror-tracing task. Saliva was collected at several time points before and after each challenge. Saliva was later assayed to extract cortisol and sAA. Child-reported depressive 
symptoms were assessed using the Child Depression Inventory, and maternal-reported internalizing problems were assessed using the Child Behavior Checklist. Multilevel modelling was conducted using Hierarchical Linear Modeling.

Results: In the TSST-C, child-reported depressive symptoms were significantly associated with a declining cortisol trajectory. Maternal-reported internalizing problems were not associated with cortisol and internalizing behaviour did not moderate the impact of sAA levels on cortisol levels (i.e., coordination) in the TSST-C. With regards to the competition challenge, maternal-reported internalizing behaviours predicted cortisol-sAA coordination, such that children with more internalizing behaviours who experienced higher sAA levels also had higher cortisol reactivity. However, there were no significant associations between child-reported depressive symptoms, cortisol reactivity, or coordination between sAA and cortisol reactivity in the competition challenge.

Conclusions: Results are discussed in the context of allostatic load and in relation to theories of physiological coordination. These findings underscore the importance of differential stressor contexts, physiological coordination, and the informant of internalizing behaviours moderating associations between internalizing behaviour and cortisol reactivity. Future research should integrate these factors in models of physiological stress and developmental psychopathology. 


\section{Acknowledgements}

I would like to thank my supervisor, Dr. Leslie Atkinson, for his mentorship and guidance throughout my graduate career. You have provided me with countless opportunities and supported me in achieving my goals, for which I am deeply grateful. I would also like to extend my gratitude to Dr. Andrea Gonzalez, for teaching me the ins and outs of biological research, patiently trouble-shooting problems, and always being available for help. I would also like to thank Dr. Karen Milligan, for her clinical and research mentorship over the years and her insightful feedback on this dissertation. Finally, I would like to thank Dr. Stéphanie Walsh Matthews and Dr. Rebecca Pillai Riddell for their willingness to contribute their time and for their insightful feedback.

I would also like to thank my fellow graduate students and the research assistants in the Biopsychosocial Lab. This research would not have been possible without the dedicated researcher team who assisted with data collection and processing. I would like to especially thank Brittany Jamieson for her guidance and support in performing statistical analyses. I would also like to thank the mothers and children who participated in this study. Thank you all for your commitment to this research.

Lastly, I would like to thank my family and friends for supporting me through the challenges and celebrating the big and small accomplishments with me. I would like to especially thank my mother. I would not be the person I am today without your unconditional love and unwavering encouragement. I dedicate this dissertation to you. 


\section{Table of Contents}

Abstract..................................................................

Acknowledgements.............................................. $\quad \mathrm{v}$

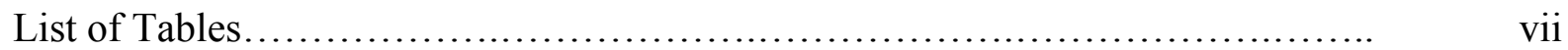

List of Figures..................................................... viii

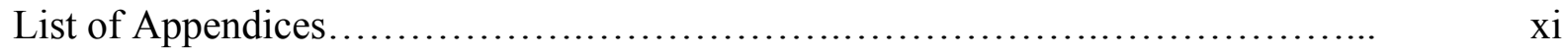

Introduction. ........................................................ 1

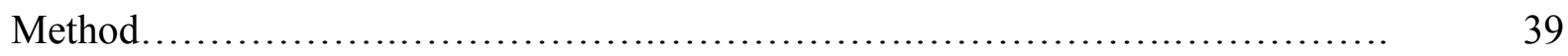

Results........................................................... 49

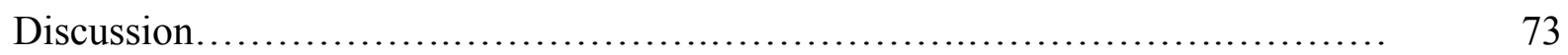

Appendices.......................................................... 99

References............................................................ 265 


\section{List of Tables}

Table 1: Summary of prior research that assesses the stressor paradigm, physiological coordination, and the rater of internalizing problems as confounds to the association between cortisol and internalizing problems................. 4

Table 2: HPA and SAM profiles of the four responsivity patterns in the ACM.... 24

Table 3: Descriptive statistics...................................... 50

Table 4: Correlations between salivary cortisol, salivary alpha amylase, and

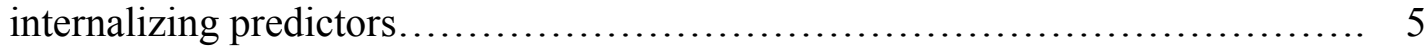

Table 5: Correlations between potential covariates and salivary cortisol......... 53

Table 6: Fixed effect estimates from the final 2-level multilevel model with child-reported depressive symptoms predicting log transformed cortisol levels in

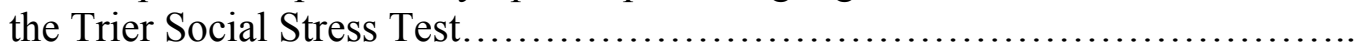

Table 7: Fixed effect estimates from the final 2-level multilevel model with maternal-reported internalizing problems predicting log transformed cortisol

Table 8: Fixed effect estimates from the final 2-level multilevel model with child-reported depressive symptoms predicting log transformed cortisol levels in the Competition challenge.

Table 9: Fixed effect estimates from the final 2-level multilevel model with maternal-reported internalizing problems predicting log transformed cortisol levels in the Competition challenge.... 


\section{List of Figures}

Figure 1: Hypothalamic-Pituitary-Adrenal Axis negative feedback loop ......... 8

Figure 2: Overlapping neurocircuitry of the Hypothalamic-Pituitary-Adrenal (HPA) Axis and Sympathetic-Adrenal-Medullary (SAM) system............... 10

Figure 3: Area under the curve with respect to ground (Panel A) and area under the curve with respect to increase (Panel B).

Figure 4: Area under the curve with respect to increase $\left(\mathrm{AUC}_{\mathrm{I}}\right)$, representing a stress response to a single challenge.

Figure 5: Salivary cortisol and salivary alpha-amylase trajectories across the Trier Social Stress Test and puzzle/mirror challenge.

Figure 6: Interaction between self-reported child depressive symptom and saliva sampling time (linear) predicting log transformed cortisol levels in the Trier Social Stress Test...

Figure 7: Interaction between self-reported child depressive problems and saliva sampling time (quadratic) predicting log transformed cortisol levels in the Trier Social Stress Test........................................................ 61

Figure 8: Interaction between maternal-reported internalizing problems and log transformed salivary alpha-amylase predicting log transformed cortisol levels in

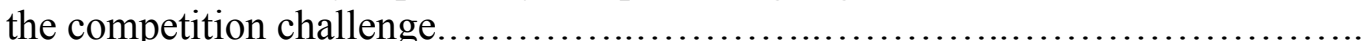

Figure 9: Visual depiction of cortisol trajectories in the Trier Social Stress Test and Competition challenge, for those with high and low self-reported depressive symptoms 


\section{List of Appendices}

Appendix 1: Trier Social Stress Test -Child Version: Story............... 99

Appendix 2: Competition Challenge - Star shape for tracing \& mirror

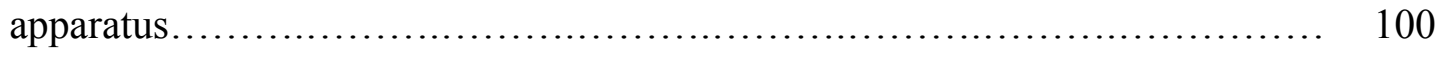

Appendix 3: Competition Challenge - Puzzle.......................... 102

Appendix 4.1: Model Equations and Results: Self-Reported Depression in the

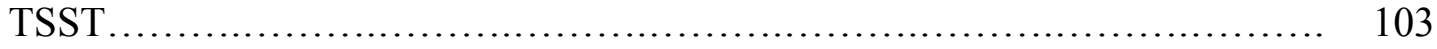

Appendix 4.2: Model Equations and Results: Maternal-Reported Internalizing in the TSST ......................................................

Appendix 4.3: Model Equations and Results: Child-Reported Depression in the Competition Challenge................................................

Appendix 4.4: Model Equations and Results: Maternal-Reported Internalizing in the Competition Challenge .............................................. 


\section{Examining associations between internalizing behaviour, cortisol reactivity, and physiological coordination, in two differentially potent challenges}

Stress physiology plays an essential role in nearly all human physical and mental health processes (Gallagher, Reid, \& Ferrier, 2009; Reynolds, 2013; Turner-Cobb, 2005) across the lifespan (Lesage et al., 2006; Levine, 2005; Meaney \& Szyf, 2005). Over the past several decades, researchers have explored the Hypothalamic-Pituitary-Adrenal (HPA) axis as a primary biological system mediating stress responses in humans. HPA activation leads to the production of the hormone cortisol, which is secreted in response to stress (Chrousos, 2009). Importantly, activation of the HPA axis can be adaptive, enabling organisms to respond efficiently and effectively to perceived threats, however, inflexible and chronic activation is neurobiologically and psychologically damaging (Chrousos, 2009; McEwen \& Seeman, 1999; Miller, Chen, \& Zhou, 2007). In particular, extensive research demonstrates that atypical cortisol responsivity is associated with internalizing behaviours during childhood and adolescence (Gunnar \& Quevedo, 2007; O'Keane, Frodl, \& Dinan, 2012). However, research associating cortisol responsivity to child internalizing behaviours is equivocal, with studies suggesting positive (Booij et al., 2013), negative (Harkness et al., 2011) and non-significant (Attwood et al., 2011) associations.

There are several confounds that potentially contribute to the inconsistencies within the cortisol literature. First, it is difficult to interpret associations between internalizing behaviours and cortisol reactivity across (and, at times, within) studies because stressor paradigms vary in potency and utility of eliciting a stress response (e.g., Gunnar, Talge \& Herrera, 2009). Second, the HPA axis is activated in coordination with the Sympathetic-Adrenal-Medullary (SAM) system, and the nature of associations between internalizing behaviours and cortisol reactivity is impacted by this physiological coordination (e.g., El-Sheikh, Erath, Buckhalt, Granger, \& Mize, 
2008). Third, associations between internalizing behaviours and cortisol reactivity vary depending on the reporter (children or parents) of internalizing behaviours (e.g., Hartman, Hermanns, de Jong, \& Ormel, 2013).

Given these confounds, the primary aim of this study is to elucidate the potential deleterious effects of internalizing behaviours on cortisol activity, controlling for the differential potency of stressor paradigms, HPA and SAM coordination, and the rater of internalizing behaviours, both methodologically and statistically.

With regards to the stressor paradigm as a confound, I will examine how internalizing behaviours are associated with cortisol reactivity in two distinct stressor paradigms, which were specifically designed to differentially elicit cortisol responsivity. For reasons discussed below, I did not include reactivity to both challenges in a single equation, but the assessment of reactivity in two challenges, albeit independently, does permit examination of generalizability of findings. In addition, I address coordination between the HPA and SAM systems.

There are several ways of measuring SAM activation, including levels of catecholamines in blood (e.g., noradrenaline, norepinephrine), as well as physiological measures (e.g., skin conductance, heart rate) (e.g., Schumacher, Kirschbaum, Fydrich, \& Ströhle, 2013). Research has shown that salivary alpha-amylase (sAA), an enzyme found in saliva, is a good marker of SAM activity and is associated with other SAM indices (Chatterton et al., 1996; Rohleder et al., 2004). In the present study I assess HPA and SAM coordination by statistically assessing sAA as a moderator of cortisol reactivity in each of these differentially potent laboratory challenges.

Lastly, I conduct separate statistical analyses to examine whether parent-reported or child-reported internalizing behaviours are differentially associated with cortisol reactivity, in both of the laboratory stressors. Although prior research has independently assessed how the 
stressor paradigm, physiological coordination, and the rater of internalizing problems may confound associations between internalizing behaviour and cortisol reactivity, rarely do studies account for a combination of confounds (see Table 1), and this is the first study to simultaneously account for all three of these factors. 
Table 1: Summary of prior research that assesses the stressor paradigm, physiological coordination, and the rater of internalizing problems as confounds to the association between cortisol and internalizing problems.

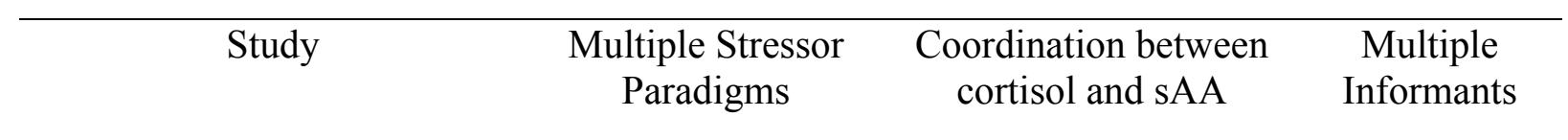

Attwood et al. (2011)

Bae et al. (2015)

$\mathrm{X}$

$\mathrm{X}$

Booij et al. (2013)

Chen et al. (2015)

El-Sheikh et al. (2008)

$\mathrm{X}$

Granger et al. (1994)

Harkness et al. (2011)

$\mathrm{X}$

Hartman et al. (2013)

X

Hill-Soderlund et al. (2015)

$\mathrm{X}$

Janssens et al. (2012)

Keenan et al. (2013)

$\mathrm{X}$

Koss et al. (2014)

X

Laurent et al. (2015)

Laurent et al. (2016)

X

$\mathrm{X}$

LeMoult et al. (2015)

Spies et al. (2011)

Spinrad et al. (2009)

X

Steeger et al. (2017)

Suzuki et al. (2013)

Vigil et al. (2010)

$\mathrm{X}$

Note: $\mathrm{X}=$ confound assessed in the study; sAA = salivary alpha amylase. 
In order to understand how the stressor paradigm, physiological coordination, and the rater of internalizing behaviours potentially confound associations between internalizing behaviours and cortisol reactivity, I will first provide an overview of the cortisol literature more broadly, discuss associations between HPA and SAM physiological systems, and then discuss internalizing behaviours in relation to HPA and SAM activity. Within these broader areas of literature review, I will highlight the importance of potential confounds, namely, the stressor paradigm, coordination between HPA and SAM systems, and the rater of internalizing behaviours.

\section{Hypothalamic-Pituitary-Adrenal (HPA) Axis}

As organisms interact with the environment, stress systems are constantly titrated in response to internal and external inputs. Following this activation, a cascade of neurological, physiological and behavioural changes ensue, with the ultimate goal of maintaining a dynamic equilibrium, known as homeostasis (Chrousos, 2009). The HPA axis is one of the most important, and heavily researched, stress regulatory systems in the human body (Kaltsas \& Chrousos, 2007). The HPA system, including its end product, cortisol, is central to physiological regulation under stressful circumstances, as well as adaptive coping on a day-to-day basis (Habib et al., 2000). The HPA axis is constantly receiving input from the external environment and responding in a dynamic way, in order to achieve neurochemical stability (Danese \& McEwen, 2012). However, for some individuals, internal and external stressors can lead to atypical activation of the HPA axis and connected neurobiological systems (e.g., SAM system), which can have devastating metabolic, immunologic, and psychological implications (Chrousos, 2009; Kaltsas \& Chrousos, 2007). 


\section{Associations between the HPA axis and the Sympathetic-Adrenal-Medullary (SAM)}

System. In order to adequately capture the stress response, it is important to understand HPA activity in the context and function of other stress networks. Despite the vast interest that cortisol has received in past decades, the HPA axis is connected to other stress networks, for example, the SAM system. The HPA and SAM stress systems are organized in a hierarchical fashion, with one or both systems being sequentially and preferentially activated depending on the nature, intensity and duration of the stressor (Del Giudice et al., 2011). For example, in many instances, strong activation of the HPA axis is not required, because the threat is managed sufficiently by the faster acting SAM system (Del Giudice et al., 2011).

SAM activity can be marked by salivary alpha-amylase. Salivary alpha-amylase (sAA) is a digestive enzyme produced by salivary glands in the oral cavity (Granger et al., 2006). Salivary alpha-amylase is a known marker of stress reactivity (Granger et al., 2006), and correlates highly with other stress indices (e.g., norepinephrine) associated with activation of the SAM system (Chatterton et al., 1996). The SAM system produces rapid and short-lasting effects. Although the SAM system is one of the initial stress systems to be activated, its effects are preparatory and can have a long-lasting impact on bodily responses (e.g., activation of cardiovascular system, maintenance of blood pressure) to stress (Ulrich-Lai \& Herman, 2009). In fact, sAA increases relatively quickly (5 to 10 minutes) after the onset of a stressor (Granger, Kivlighan, El-Sheikh, Gordis, \& Stroud, 2008; Nater et al., 2005, 2006). In comparison, cortisol peaks approximately 20 to 40 minutes after the onset of a stressor (Dickerson \& Kemeny, 2004). Importantly, activation of each of these systems is integrated and co-regulated through overlapping neurobiological circuitry. 
Neurocircuitry of the HPA axis. The HPA response is initialized by paraventricular nuclei (PVN) activation in the hypothalamus (see Figure 1). PVN trigger the release of two hormones, corticotrophin-releasing hormone (CRH) and arginine vasopressin (AVP) (Ulrich-Lai \& Herman, 2009). CRH then generates the release of adrenocorticotropic hormone (ACTH), which is transported to the adrenal cortex of the adrenal gland, where it produces glucocorticoid hormones, one of which is cortisol (Gunnar \& Quevedo, 2007). Importantly, the HPA axis includes a negative feedback loop to initiate stress recovery and prevent prolonged activation (Guilliams \& Edwards, 2010). When cortisol reaches an optimal level it binds to glucocorticoid receptors (GRs), which activate pathways back to the PVN and stop the production of CRH and ACTH (Sapolsky, Romero, \& Munck, 2000). Thus, GRs are particularly important for stress recovery and the minimization of cortisol overproduction, which could potentially have deleterious long-term effects (Guilliams \& Edwards, 2010). 


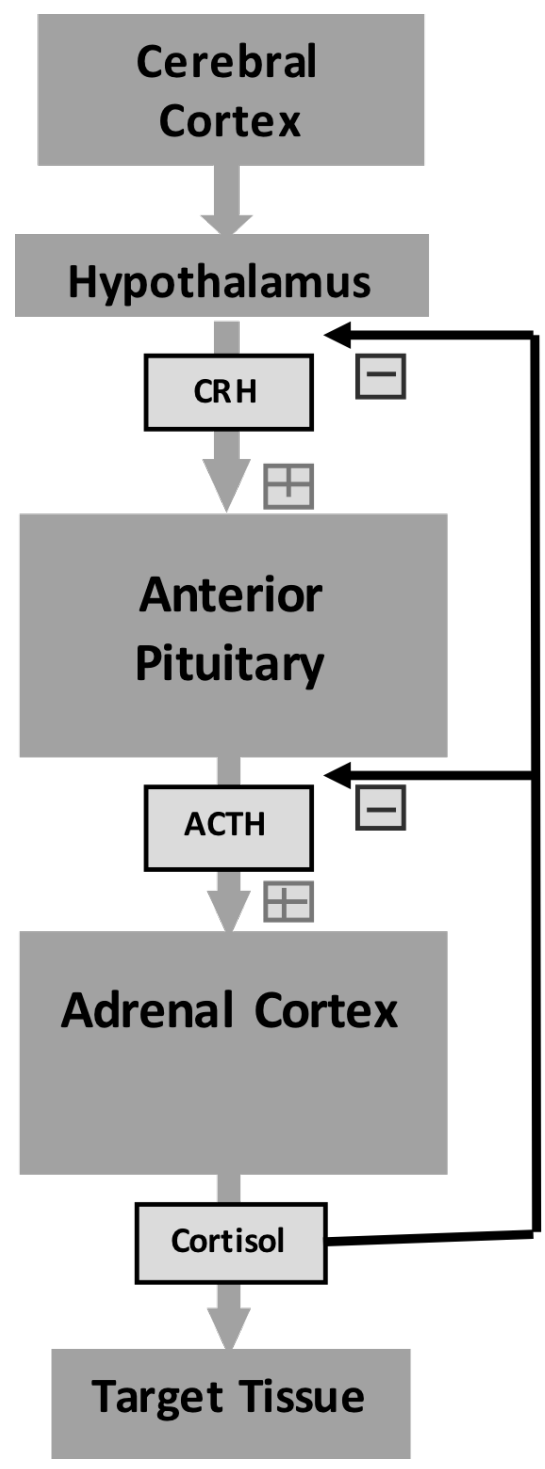

Figure 1. Hypothalamic-Pituitary-Adrenal Axis negative feedback loop. 
Overlapping neurocircuitry of HPA and SAM systems. The sympathetic response is coordinated by the locus coeruleus (LC). There are two main routes of sympathetic activation. The first involves direct activation of noradrenergic organs, and the second involves activation of the adrenal medulla, which triggers the release of catecholamines including epinephrine (adrenaline) and norepinephrine into the bloodstream (Goldstein \& Kopin, 2008; Gunnar \& Vazquez, 2006). It is the activation of the adrenal medulla and the resulting hormonal pathway that comprises the SAM system (Del Giudice, Ellis, \& Shirtcliff, 2011).

Although the HPA and SAM systems are comprised of distinct neurocircuitry (as discussed above), the activation of both systems is coordinated by shared structures and overlapping hormonal products (Figure 2). In terms of anatomy, the amygdala initiates and controls both HPA and SAM responses (Ganzel et al., 2010; Gold \& Chrousos, 2002; Herman et al., 2003). In response to perceived stress, the amygdala stimulates PVN in the hypothalamus as well as the LC, thereby initializing the HPA responses (Kaltsas \& Chrousos, 2007). In addition, the medial prefrontal cortex (mPFC) acts as a top-down coordinator of both HPA and SAM responses, with some components of the MPFC stimulating HPA and SAM responses, and other components inhibiting these responses (e.g., reducing the HPA response by regulating the duration of glucocorticoid secretion) (Ulrich-Lai \& Herman, 2009). Given the vast interconnections of the PFC with other brain structures (e.g., amygdala, hippocampus), the PFC appears to act as the principal coordinator of HPA and SAM neurobiological stress reactivity (Ulrich-Lai \& Herman, 2009). 


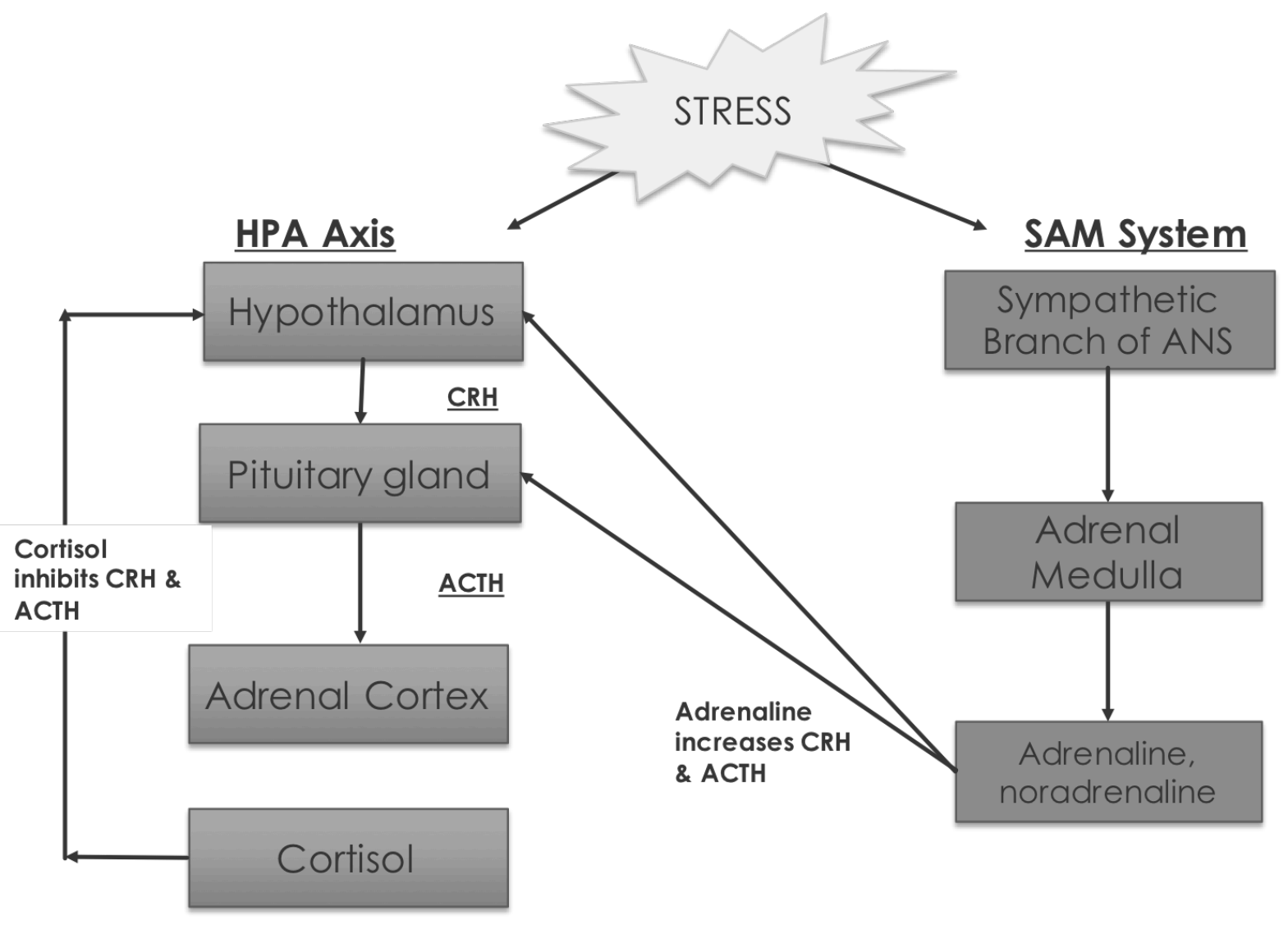

Figure 2. Overlapping neurocircuitry of the Hypothalamic-Pituitary-Adrenal (HPA) Axis and Sympathetic-Adrenal-Medullary (SAM) system. 
In addition, $\mathrm{CRH}$ neurons in the hypothalamus and noradrenergic neurons are activated and inhibited by shared neurotransmitters (Stratakis \& Chrousos, 1995). These neurons also have reciprocal connections, permitting modulation across HPA and SAM systems (Stratakis \& Chrousos, 1995). For instance, noradrenergic neurons in the LC can stimulate the release of $\mathrm{CRH}$ in the PVN, but circulating cortisol inhibits noradrenergic neuronal activity in the LC and reduces CRH production (Habib et al., 2001; Valentino et al., 1998; Sapolsky et al., 2000). It is this overlapping neurocircuitry as well as the complementary activation that suggests that these two stress systems operate in a coordinated fashion.

Cortisol indices. Cortisol is commonly measured in terms of acute responsivity to laboratory and naturalistic stressors. Over decades of research, a plethora of cortisol indices have been used to represent baseline levels, total output, reactivity and recovery (Khoury et al., 2015). Many previous studies include fewer than four saliva collection points, thus limiting the ability examine cortisol trajectories and increasing the reliance on point estimates. Of the numerous point estimates used to characterize cortisol responses to acute challenge, total cortisol output and cortisol reactivity, indexed by area under the curve with respect to ground and increase $\left(A U C_{G}\right.$ and $\mathrm{AUC}_{\mathrm{I}}$, respectively), appear to be the best indices of cortisol output and cortisol change (Khoury et al., 2015). Area under the curve with respect to ground $\left(\mathrm{AUC}_{\mathrm{G}}\right)$ represents total cortisol output; it assesses both the difference between single measurement points and the distance of measurement points from the ground, thereby taking into account the absolute baseline level (Fekedulegn et al., 2007; see Figure 3). Area under the curve with respect to increase $\left(\mathrm{AUC}_{\mathrm{I}}\right)$, measures cortisol change from baseline over subsequent time points, and does not take into account baseline levels (Fekedulegn et al., 2007; Figure 3). $\mathrm{AUC}_{\mathrm{I}}$ is a reliable indicator of cortisol change and thus captures the stress system's response to 
environmental challenge (Atkinson et al., 2016; Fekedulegn et al., 2007; Khoury et al., 2015). However, measurement issues arise when using $\mathrm{AUC}_{\mathrm{I}}$ and other point estimates. For instance, the same $\mathrm{AUC}_{\mathrm{I}}$ value can indicate multiple types of cortisol trajectories (Atkinson, Jamieson, Khoury, Ludmer, \& Gonzalez, 2016; Fekedulegn et al., 2007). As shown in Figure 4, an $\mathrm{AUC}_{\mathrm{I}}$ value of 3.35 can represent two very distinct patterns of cortisol reactivity, one indicating a rapid increase followed by an intense decline back to baseline, while the other representing a more blunted increase without a return to baseline. This ambiguity makes it difficult to determine the meaning of $\mathrm{AUC}_{\mathrm{I}}$ levels within a single laboratory challenge and limits comparisons of cortisol reactivity findings across studies. 


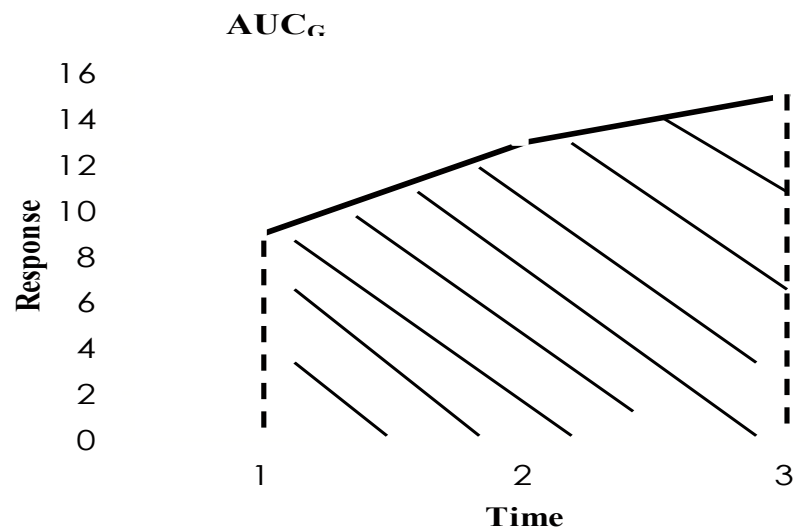

A

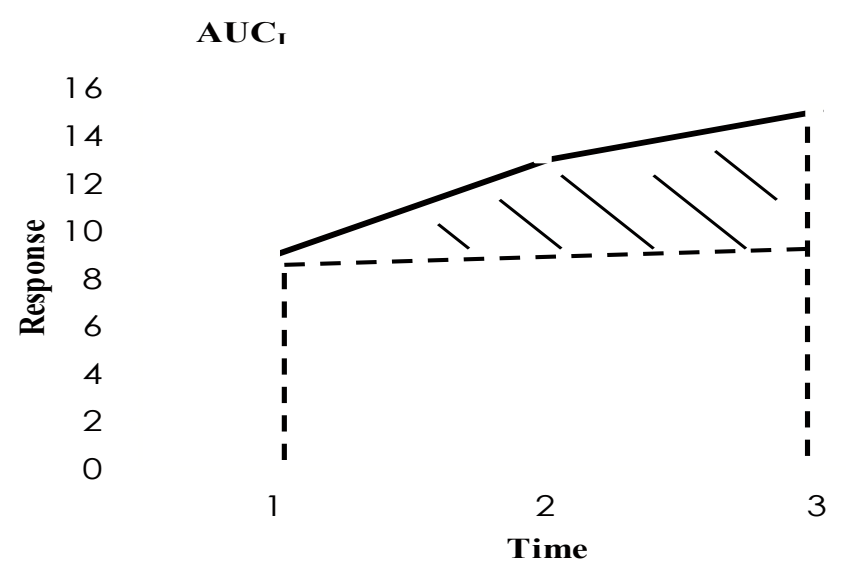

B

Figure 3. Area under the curve with respect to ground (Panel A) and area under the curve with respect to increase (Panel B). 


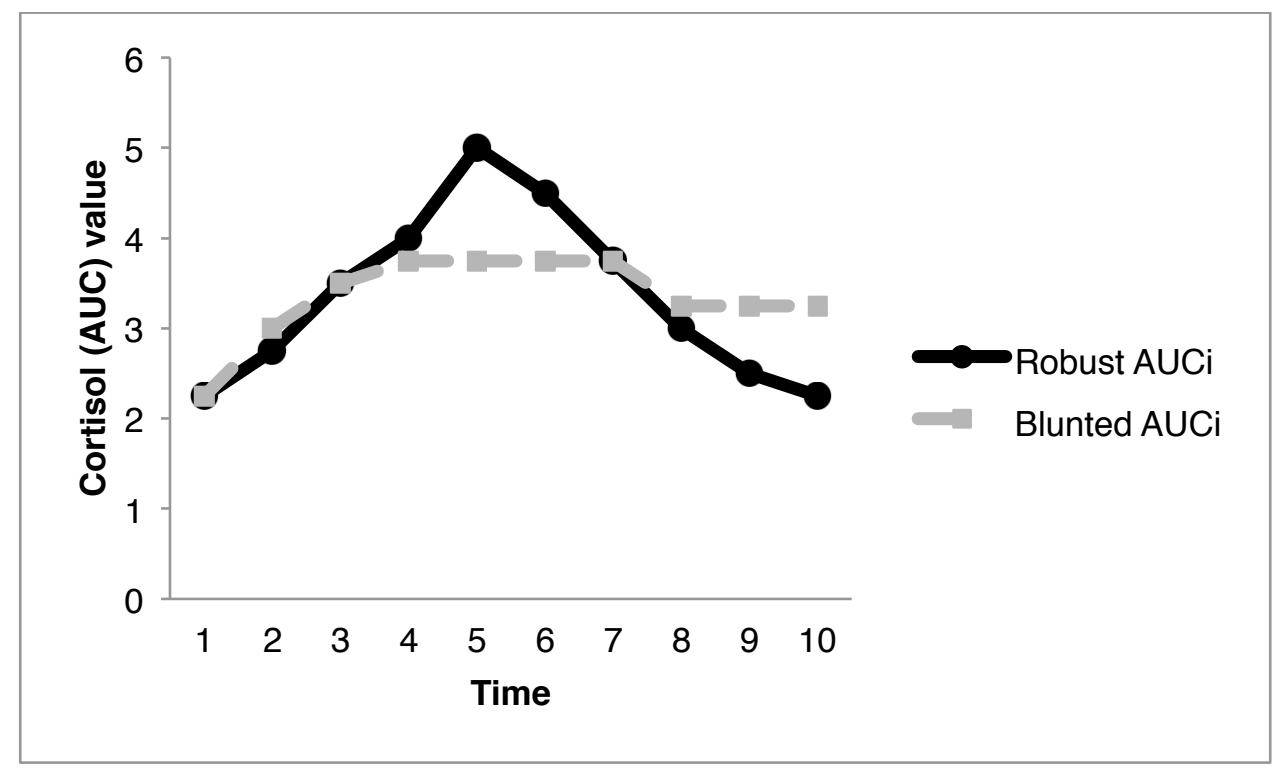

Figure 4. Area under the curve with respect to increase $\left(\mathrm{AUC}_{\mathrm{I}}\right)$, representing a stress response to a single challenge. Robust $\mathrm{AUC}_{\mathrm{I}}$ with return to baseline and blunted $\mathrm{AUC}_{\mathrm{I}}$ without return to baseline, both $\mathrm{AUC}_{\mathrm{I}}$ values are equivalent (3.35). 
Taken together, it is difficult to interpret what constitutes an adaptive cortisol response, especially when the majority of studies measuring cortisol responses use different point estimates, which have ambiguous interpretative value. More recently, cortisol has been indexed by trajectories across different time points and stressor paradigms (e.g., Laurent, Ablow, \& Measelle, 2012), providing a more refined picture of the dynamic stress response process that encompasses acute cortisol reactivity and recovery (as discussed below). In this study, I adopt a trajectory approach. But in addition to using more refined indices of cortisol, a more refined analysis of the HPA homeostasis process is also required to understand adaptive stress reactivity.

\section{Achieving Allostasis: Flexibility in Stress Responsivity}

Stress responsivity involves a complex set of dynamics, which when functioning optimally can lead to regulation of the stress system and maintenance of other bodily functions (metabolism, immune system function etc.), but when functioning atypically can lead to a plethora of adverse neurobiological, immunological, and psychological effects (Danese \& McEwen, 2012). Allostasis refers to the biological changes that function to maintain neurobiological stability (Sterling \& Eyer, 1988; Goldstein \& McEwen, 2002). Allostatic load refers to the 'wear and tear' caused by too much stress or ineffective management of allostasis (i.e., the stress response persists when stress is over, and/or the stress response is not turned on adequately when needed) (McEwen, 2007). McEwen (1998) outlined different stress responses to frequent and repeated stressors, some of which are adaptive while others are associated with allostatic load. An adaptive response to a single stressor includes abrupt reactivity, followed by recovery (return to baseline levels). An adaptive response to repeated stressors involves an initial activation of the stress response followed by recovery, and with repeated stressors (or "hits") the magnitude of this response attenuates. In contrast, lack of adaptation is seen either as a) 
a prolonged (i.e., sustained) response (including a lack of recovery), which results from an inability to shut off the allostatic response after stress is terminated, or b) an inadequate or blunted response, which results in increased activation of compensatory or other stress mediators (McEwen, 1998). Thus a 'healthy' stress response depends on elasticity; in the case of the HPA axis, the ability to a) produce a pronounced cortisol reaction when threatened and b) down-regulate this response, that is, maintain a stable level of cortisol when no longer under acute threat (McEwen \& Seeman, 1999).

To date, very few studies have measured cortisol reactivity across multiple challenges, and thus do not assess allostatic load as it pertains to repeated stress, across similar or distinct contexts (Atkinson et al., 2016). It is important to measure stress reactivity across distinct contexts because a) laboratory paradigms differ in their ability to elicit HPA and SAM responses, and thus potentially influence HPA and SAM coordination; and b) individual differences (such as internalizing behaviour) are associated with stress reactivity differently across contexts. Below I present three lines of research that underscore the importance of assessing cortisol reactivity in distinct challenges. The first type of evidence is offered through literature reviews, which suggest that stressor paradigms used across different studies vary in potency and capability to evoke cortisol responses (i.e., Dickerson \& Kemeny, 2004; Gunnar et al., 2009; Jansen, Beijers, Riksen-Walraven, \& de Weerth, 2010). The second line of research is in the form of single studies that directly compare the effectiveness of multiple stressor paradigms to elicit cortisol responses, allowing for the assessment of intra-individual variability in stress responsivity (e.g., Laurent, Ablow, \& Measelle, 2012). This second line of research examines overall group differences in cortisol reactivity, assessed across multiple, and differentially potent laboratory paradigms. Although this body of research examines cortisol reactivity within the same subjects, these studies do not directly assess how individual differences might impact one's ability to adjust their physiological stress reaction, in response to the potency of each specific challenge. The 
third line of research directly examines cortisol flexibility, that is, how individual differences (e.g., on a psychological variable) moderate one's ability to titrate their physiological stress response in accordance with the potency of divergent stressors (e.g., Atkinson et al., 2016). These three lines of research are discussed below.

Differentially potent laboratory challenges. Extensive research has been conducted using a variety of different laboratory challenges, all aimed to elicit stress reactivity. Interestingly, over time, this research has revealed that these laboratory challenges diverge in their effectiveness to evoke HPA responsivity. A systematic review (Gunnar et al., 2009) and a meta-analysis (Jansen et al., 2010) outlined the differential effectiveness of common stressor paradigms to elicit cortisol responses across development. Although effect sizes were not directly compared, results from Jansen et al. suggest that parent-child separation challenges may elicit a stronger cortisol response $(d=.34)$ for infants compared to frustration challenges $(d=.13)$. In addition, social-evaluative threat challenges are the most potent for older children (Gunnar et al., 2009) and adults (Dickerson \& Kemeny, 2004). Similarly, in adults, emotion-induction paradigms are less potent, eliciting a negative cortisol response (Dickerson \& Kemeny, 2004). Taken together, this research suggests that across development, stronger cortisol responses are elicited by threatening challenges, whereas weaker responses are elicited by emotionbased challenges.

In addition to these systematic reviews, researchers have begun to utilize multiple stressors within a single study to examine whether some paradigms evoke stronger cortisol responses. To examine whether the paradigms had a differential effectiveness to elicit a cortisol response, Stroud and colleagues (2009) employed a between-subject design wherein adolescents were assigned to complete one of two different laboratory challenges. By examining inter-individual, between-stressor variability, Stroud et al. (2009) showed that adolescents who participated in a Trier Social Stress Test (TSST; 
Kirschbaum, Pirke, \& Hellhammer, 1993) had higher cortisol responses, compared to the adolescents who participated in a peer rejection stressor. However, given that this study examined between-subject variability, the interpretation of these results are limited by the numerous individual factors that vary across participants and potentially contributed to distinct responses within each stressor.

In addition, researchers have begun to employ designs wherein differentially potent stressor paradigms are used to examine intra-individual, between challenge variability. These studies use within-subject designs to demonstrate that stressor paradigms elicit distinct cortisol responses, depending on the potency of each stressor. However, these studies do not examine whether individual differences might impact participants' ability to titrate their stress response across challenges. Instead this line of research simply shows that the same group of individuals who participate in multiple challenges, demonstrate variant cortisol responses. In this line of research, allostasis is operationalized as a variable response across challenges, i.e., strong response to potent challenge and diminished response to less potent challenge. For example, Maruyama et al. (2012) showed that healthy adults, who participated in both the TSST and an electrical stimulation stressor, demonstrated higher cortisol responses in the TSST compared to electrical stimulation stressor. In a sample of mother-infant dyads, Laurent, Ablow, and Measelle (2012) also examined intra-individual, between challenge variability. Laurent et al. (2012) demonstrated that infants had a strong cortisol response to a separation stressor and a less pronounced response to clean up task. These studies further advance the literature by using within-subject designs to examine intra-individual variability in stress responses. However, this research does not directly examine psychological variables that could potentially impact an individual's ability to titrate their cortisol responses across differentially evocative stressor paradigms.

Examining individual differences as predictors of flexibility in cortisol responses, assessed across differentially potent laboratory paradigms. In addition to considering stressor potency 
differences, it is important to consider whether intra-individual factors impact flexibility of stress responses across distinct stressors, on the assumption that some individuals are better able to titrate response better than others. As outlined by Atkinson and colleagues (2016), a "flexible response is [one that is] titrated in an ecologically appropriate manner to the degree/type of challenge" (p. 4). In accordance with this definition, several criteria must be met to adequately capture flexible stress responsivity. First, multiple and differentially potent challenges must be used with the same sample of individuals to draw conclusions regarding intra-individual variability in stress reactivity between challenges. Second, differential stress reactivity across challenges must be predicted (i.e., moderated) by a relevant feature of the individual. Further to this second requirement, the studies discussed below demonstrate flexible stress responsivity using statistical comparisons to show that individuals differentially titrate cortisol reactivity to the nature of the challenge, depending on some relevant feature pertaining to themselves or their environment.

Atkinson et al. (2013) showed that maternal sensitivity predicted infants' cortisol flexibility across challenges. That is, infants of more sensitive mothers show adaptive cortisol flexibility - a robust increase in cortisol in response to a potent and threatening separation challenge and a robust decline in cortisol in response to a less potent frustration challenge. In contrast, infants of less sensitive mothers showed less dramatic down- and up-regulation, respectively. Atkinson et al. (2013) argued that flexible cortisol reactivity across variant challenges is central to adaptive responses in infants, and maternal factors (i.e., sensitivity) influence this flexibility (Atkinson et al., 2013). Utilizing the same sample, Ludmer et al. (2015) replicated these results by examining the interaction between maternal depressive symptoms and infant genetics across these two differentially potent challenges. Findings indicated that infants whose mothers endorsed more maternal depressive symptoms show reduced cortisol flexibility across challenges, but only if the infants were also genetically vulnerable (i.e., had 
high-susceptibility dopaminergic genotypes). Additional research is needed to understand cortisol flexibility across different stressors in samples of children and adults. Given the fact that distinct challenges evoke differential response, this study explored the impact of child internalizing problems on cortisol reactivity in response to differentially potent stressors.

\section{Coordination between HPA and SAM Systems}

As discussed above, overlapping neurocircuitry as well as complementary neurobiological activation suggests that the HPA and SAM stress systems operate in a coordinated fashion. As stated by Bauer and colleagues (2002), "the SAM and HPA systems are anatomically and physiologically connected in the central nervous system, although much remains to be determined about the nature of the systems' coordination at the physiological response level as well as the behavioral consequences of this coordination or its disruption" (p. 105). More recently, researchers have emphasized the need for concurrent assessment of HPA and SAM systems in order to decipher the nature of coordination and its' association with behavior and psychopathology (Bauer et al., 2002; Granger \& Fortunato, 2008). Concurrent assessment of cortisol and sAA might improve our understanding of HPA dysregulation, allostatic overload, and associated adverse health outcomes. Preliminary evidence suggests that the ratio of cortisol to sAA is a better indicator of stress dysregulation compared to either index alone (Ali \& Pruessner, 2012). However, the combined effects of the HPA and SAM circuits are quite complex, and multiple theories have been proposed to explain the nature of the coordination between these two systems.

For decades, research has examined how atypical HPA activity impacts behavioural, emotional and social experiences across development. Bauer and colleagues (2002) were among the first to propose that associations between HPA activity and social-emotional outcomes are likely impacted by coordination between HPA and SAM systems. Bauer and colleagues (2002) proposed a comprehensive 
outline of how HPA and SAM are coordinated, suggesting that cross-system activation can be coordinated in either an additive or interactive model. The additive model suggests that HPA and SAM activation work in conjunction in an inverted "U" shape fashion, such that a moderate level of arousal in both systems is optimal. Further, low activation in one system paired with high activation in another (i.e., asynchronous activity) is also adaptive, whereas high or low activation in both systems (i.e., synchronous activity) is maladaptive (Bauer et al., 2002). However, HPA and SAM activity are nonredundant and often complementary. As such, the interactive model proposes that optimal functioning occurs when a balance between systems is achieved. The interactive model suggests that coupled low, moderate, or high activation in both systems is most adaptive, whereas a mismatch (high activation in one system and low activation in another) is maladaptive (Bauer et al., 2002). Chen and colleagues elaborated on the additive and interaction models, stipulating statistical techniques for specifying interaction models (Chen, Raine, Soyfer, \& Granger, 2015). That is, interactive models can be measured using ratio or interactive terms, where the ratio approach assumes a unidirectional association between the two systems, in contrast the interaction model, which does not specify the strength or direction of associations. The interaction model allows for a unidirectional or bidirectional association between systems (Chen et al., 2015). Chen et al. suggest that the ratio approach should be used when the two systems have opposite/suppressive effects and the interaction approach should be used when the strength and direction of one system's effect on an outcome is dependent on the level of the other system. Reeves et al. (2016) elaborated on this theory to suggest that the ratio approach is best used when HPA and SAM coordination is the dependent variable of interest (because the HPA/SAM ratio can be computed as a single variable), whereas the interaction approach is best used when examining coordination as a predictor (Reeves, Fisher, Newman, \& Granger, 2016). In line with this latter 
approach, I used an interaction method in the present study to assess the effect of sAA on cortisol as an outcome.

The Adaptive Calibration Model (ACM; Del Giudice et al., 2011) is one model that intricately connects environmental experiences across development and stress system coordination. The ACM explains individual differences in stress responsively by drawing extensively on evolutionary and developmental biology. At its core, ACM postulates that individual differences in stress responsivity result from conditional adaptation, which is an evolutionarily-based ability of an organism to modify its phenotypic developmental trajectory to match environmental conditions. ACM proposes four resulting patterns of coordination: sensitive, buffered, vigilant, and unemotional (see Table 2, adapted from Del Giudice et al., 2011). The sensitive phenotype involves high levels of HPA reactivity and relatively moderate levels of SAM reactivity, which allows the individual to receive environmental feedback and adjust system activation accordingly. It is predicted that the sensitive phenotype evolves from a safe, protected, and supportive environment. This is partially supported by research suggesting that infants of more sensitive mothers have higher cortisol reactivity (Blair et al., 2006). However, theoretical and empirical findings suggest that "high cortisol reactivity" depends on the type of challenge (e.g., Gunnar et al., 2009; Jansen et al., 2010), and is most accurately indexed by cortisol flexibility, measured across differentially potent challenges (Atkinson et al., 2016). To this end, more comprehensive support of the sensitive phenotype comes from research showing that sensitive caregiving predicts high cortisol reactivity in a potent separation challenge and steeper cortisol declines in a less potent frustration challenge (Atkinson et al., 2013). The buffered phenotype is characterized by moderate HPA activity and low/moderate SAM activity. This phenotype is hypothesized to evolve in moderately stressful environments, wherein repeated exposure to stress early in life results in downregulation of the HPA and SAM systems. The vigilant pattern involves high reactivity of both the HPA 
and SAM systems. This phenotype is proposed to evolve from highly stressful environments that consist of repeated physical and social threats. The vigilant phenotype differs from the sensitive phenotype in that it is marked by slower HPA recovery to threat (Gunnar \& Vazquez, 2006; Pruessner et al., 1997), and hypocortisolism in response to long-term stress. Lastly, the unemotional profile is marked by blunted HPA and SAM activity. This profile is associated with severe and chronic stress early in life (Gunnar and Vazquez, 2006; Gustafsson et al., 2010; Tarullo and Gunnar, 2006). Overall, the literature presented thus far suggests that 1) HPA reactivity, in particular cortisol flexibility (measured across dissimilar laboratory challenges), is an important index of allostasis, and 2) HPA activation is intricately associated with SAM activation. The next section will explore whether HPA and SAM coordination may differ depending on the type of stressor paradigm used to elicit these responses. 
Table 2. HPA and SAM profiles of the four responsivity patterns in the ACM

\section{Responsivity Patterns}

\begin{tabular}{lcccc}
\hline Physiological profile & Sensitive & Buffered & Vigilant & Unemotional \\
SNS & High/moderate & Low/moderate & High & Low \\
HPA & High & Moderate & High & Low
\end{tabular}

Note: $\mathrm{ACM}=$ Adaptive Calibration Model; SNS = Sympathetic Nervous System; HPA = Hypothalamic-Pituitary-Adrenal axis; table adapted from Del Giudice et al. (2011) 
Differential situational triggers of HPA and SAM activation. The relative differential activation of the HPA and SAM systems is likely related to the nature of the challenge (Laurent, Powers \& Granger, 2013; Lundberg \& Frankenhaeuser, 1980). It has been theorized that the HPA axis responds intensely to threatening situations, whereas the SAM system responds more strongly to general challenge (Frankenhauser, 1982; Nigg, 2006). The meaning of the response within each of these systems also differs; HPA activation is highly related to imminent threat or direct distress, whereas the SAM system is not associated with distress or negative affect, and is more so associated with general arousal implied during effortful challenge (Laurent et al., 2013; Lundberg \& Frankenhaeuser, 1980). In addition, HPA activation is prominent when the stressor has a socialevaluative component and is perceived as unpredictable and uncontrollable, whereas SAM activation is believed to be associated with controllable challenges (Flinn, 2006; Frankenhaeuser, Lundberg, \& Forsman, 1980; Lundberg \& Frankenhaeuser, 1980; Peters, Godaert, Ballieux et al., 1998). In general, laboratory stressors differ in the degree to which they elicit stress responses. More importantly, the degree to which a stressor elicits a stress response may differ depending on the type of stress system activated (i.e., HPA vs. SAM); that is, some stressors may be highly potent for eliciting HPA, but not SAM, activation, and vice versa. Therefore, when investigating HPA activity across multiple, and differentially potent challenges, it is important to concomitantly examine how coordination with the SAM system may influence this HPA flexibility.

Although theory suggests that HPA and SAM systems are activated by different stressors, empirical studies that examine the differential potency of stressors to evoke cortisol and sAA responses show mixed findings. An initial study conducted by Lundberg and Frankenhauser (1980) found that a self-paced (i.e., controllable) reaction-time task elicited strong noradrenaline, but not cortisol, reactivity, whereas a vigilance task elicited strong noradrenaline and cortisol reactivity. Based on these 
preliminary findings, Lundberg and Frankenhauser (1980) suggested that the SAM system is more strongly activated by controllable and effortful tasks, whereas the HPA axis is activated by distressing and effort-demanding tasks. Additional studies have employed different stressors, often resulting in equivocal findings in regard to the differential activation of the HPA and SAM systems. For example, research utilizing the TSST (Kirschbaum, Pirke, \& Hellhammer, 1993) produced equivocal findings; some studies indicated that this paradigm elicited a stronger cortisol compared to sAA response (Giles et al., 2014), while others demonstrated that this paradigm produced non-significant differences in cortisol and sAA responses (e.g., Kramer et al., 2012). A recent study of healthy men examined cortisol and sAA responses to four stressors, the TSST, the Stroop challenge (Stroop, 1935), the Cold Pressor Task, and a physical exercise, bicycle ergometer test (Skoluda et al., 2015). This study demonstrated that the highest cortisol response was evoked by the TSST, whereas the highest sAA response was evoked by the physical exercise ergometer test. Similarly, Laurent et al. (2012) found that infants demonstrated a strong cortisol response to a separation stressor and a less pronounced response to clean up task; in contrast, infants demonstrated a strong sAA response to the clean-up task and a less pronounced sAA response to the separation stressor.

This preliminary research suggests that cortisol and sAA responses may be sensitive to different types of stressors. Some of the aforementioned research on cortisol and sAA reactivity suggests that some socially threatening stressors evoke a stronger cortisol (relative to sAA) response, whereas other (i.e., effortful challenge) stressors evoke a stronger sAA response. However this research is in its infancy and should be interpreted cautiously. Nonetheless, this literature suggests the possibility that coordination between cortisol and sAA may differ across these two categories (i.e., socially threatening vs. competition) of stressor paradigms. 
Thus far, I have argued that the HPA axis is a primary stress network with widespread health implications. In association, I presented preliminary theoretical and empirical evidence suggesting that a key component of allostasis and allostatic load is inflexible cortisol reactivity, as assessed across differentially potent stressors. Further, given the associations between HPA activity and SAM activity, which likely differ across distinct challenges, I proposed that sAA levels might confound cortisol flexibility findings.

In addition to the factors discussed above, features of the individual (child, in the case of the present study) influence stress reactivity. In prior research, although the overall sample may not show a robust cortisol increase in response to a given challenge, some individuals (e.g., those with a particular temperament) demonstrated a strong cortisol response (Gunnar, Brodersen, Krueger, \& Rigatuso, 1996; Nachmias, Gunnar, Mangelsdorf, Parritz, \& Buss, 1996). Beginning in childhood and continuing through adolescence and adulthood, individual psychological factors can "impact the internal homeostatic state of an individual", and thus have widespread impact on stress reactivity (Tofoli et al., 2011, p. 229). As such, the following section will focus on how internalizing difficulties, a prevalent set of mental health problems, are associated with HPA activity.

\section{Childhood Internalizing Behaviours}

Internalizing behaviours are a constellation of difficulties, including depression, withdrawal, anxiety, and somatic symptoms, which lead to significant distress and have implications for social and psychological adjustment throughout development (Caspi, Bem, \& Elder, 1989). Internalizing behaviours are a set of common mental health problems that occur across the lifespan, including childhood and adolescence (Chavira, Stein, Bailey, \& Stein, 2004; Costello, Mustillo, Erkanli, Keeler, \& Angold, 2003). Early internalizing behaviours increase risk for the development of more severe disorders in adolescence and adulthood (Barber \& Olson, 2004; Graber \& Sontag, 2009; Lopez, Turner, 
\& Saavedra, 2005; Twenge \& Nolen-Hoeksema, 2002). Furthermore, subcomponents of internalizing behaviours, such as anxiety and depression, often co-occur throughout childhood and adolescence (Axelson \& Birmaher, 2001; Essau, 2008; Garber \& Weersing, 2010), with comorbidity estimates reaching up to 75\% (Angold, Costello, \& Erkanli, 1999; Avenevoli, Stolar, Dierker, \& Merikangas, 2001; Yorbik, Birmaher, Axelson, Williamson, \& Ryan, 2004). Importantly, individual differences in internalizing behaviours are known to be associated with stress physiology, namely HPA activity. Before examining the specific associations between internalizing behaviours and cortisol reactivity, it is important to consider moderators of this association. Below I highlight that, similar to the broader HPA literature, the literature examining internalizing behaviours in relation to cortisol reactivity has important confounds, namely the stressor paradigm used to evoke cortisol reactivity, the informant of internalizing behaviours (because parent and child ratings are often discrepant), and coordination between cortisol and sAA.

Child internalizing behaviours and HPA activity. Extensive research suggests that children who experience more internalizing behaviours are more likely to evince atypical HPA reactivity (ElSheikh et al., 2008; Halligan et al., 2007). The association between internalizing behaviours and atypical HPA activity has been shown concurrently (e.g., Harkness et al., 2011; Ulrike, Reinhold, \& Dirk, 2013) and longitudinal research demonstrates that atypical cortisol puts children at higher risk to later develop depressive problems (Booij, Bouma, de Jonge, Ormel, \& Oldehinkel, 2013; Hailligan et al., 2007; LeMoult et al., 2015). Research examining diurnal cortisol levels, demonstrates higher levels of diurnal cortisol in samples with higher self-reported (Van den Bergh \& Claste, 2009) and parentreported (LeMoult et al., 2015) internalizing behaviours. However, the direction of the effect of internalizing behaviours and HPA activity in laboratory experiments is equivocal. In adults, it is well accepted that depression is associated with cortisol hyperactivity (Young et al., 2000), however, studies 
with children and adolescents indicate that higher self- and parent-reported internalizing behaviours are associated with both elevated (e.g., Booij et al., 2013) and blunted (e.g., Harkness et al., 2011) cortisol levels. The distinct stressor paradigms utilized, coordination between cortisol and sAA, as well as the reporter (parent or child) of internalizing behaviours, might explain these inconsistent findings.

Internalizing behaviours in relation to cortisol reactivity in the TSST-C. The association between child internalizing behaviours and cortisol reactivity likely depends on the challenge used to elicit stress, given that laboratory paradigms differ substantially in their effectiveness in eliciting cortisol reactivity. Here I discuss how internalizing behaviours are associated with cortisol reactivity across differentially potent laboratory challenges. When examining child and adolescent cortisol reactivity, the majority of studies utilize the child-version of the TSST (TSST-C; Buske-Kirschbaum et al., 1997), which is a developmentally appropriate social (telling a story in front of judges) and cognitive (arithmetic calculations, also before judges) performance task. The TSST-C typically elicits a strong cortisol response followed by a return to baseline (Gunnar et al., 2009). Given the socialcognitive threat involved in this challenge, a strong cortisol response followed by a decline is believed to be typical and adaptive. Although the TSST-C is known to elicit cortisol reactivity, individuals with higher internalizing behaviours show distinct cortisol responses compared to those with lower levels of internalizing behaviours. For example, adolescents with high self-reported depressive symptoms demonstrated a blunted cortisol response during the TSST-C (Harkness et al., 2011). Interestingly, the chronicity of depressive symptoms had a curvilinear association with cortisol reactivity in a similar social-evaluative stressor, such that adolescents with chronic self-reported depressive symptoms demonstrated blunted cortisol reactivity (Booij et al., 2013). Importantly, the majority of the studies to date that assess internalizing behaviours in relation to cortisol reactivity in the TSST-C, focus on samples of adolescents. Fewer studies examine these associations in middle-childhood samples. 
Overall, this literature suggests that self-reported depressive symptoms are associated with blunted cortisol reactivity in the TSST-C.

\section{Internalizing behaviours in relation to cortisol reactivity in other (non-TSST-C) stressors.}

Similar to studies using the TSST-C, children with clinically elevated and sub-clinical depressive symptoms had blunted cortisol reactivity during challenges deemed to be mildly stressful (Suzuki et al., 2013). In addition, in response to the Cold Pressor Task (CPT), female adolescents with higher depressive symptoms (based on semi-structured clinical interviews of both children and mothers) demonstrated blunted reactivity compared to the rest of the sample (Keenan et al., 2013). In this study, only female adolescents were included to avoid potential sex differences, which typically become present after the onset of puberty (Stroud, Papandonatos, Williamson, \& Dahl, 2011). Other studies have implemented a conflict discussion task to assess cortisol reactivity. Spies et al. (2011) found that adolescents who reported higher levels of internalizing behaviours, demonstrated blunted cortisol responses during a conflict discussion task. In contrast, Steeger et al. (2017) did not find a significant association between maternal-reported internalizing behaviours and adolescents' cortisol response during a conflict discussion task. Similarly, Granger, Weisz and Kauneckis (1994) found that childreported depressive symptoms were not significantly associated with cortisol reactivity during a conflict discussion task. Taken together, these findings suggest somewhat equivocal associations between internalizing difficulties and cortisol reactivity in various non-TSST stressors.

The existing literature suggests that adolescents with internalizing behaviours appear to have blunted cortisol reactivity in response to the potent TSST-C. However, there are more contradictory findings when examining internalizing behaviours in the context of less potent stressors (e.g., problem solving task). Importantly, the majority of this research has been conducted with samples of adolescents, and thus it remains unclear how the age of the sample might impact associations between 
internalizing behaviour and cortisol reactivity, measured across different stressor contexts. Thus, based on the existing literature, it is premature to draw conclusions about the association between internalizing behaviours and cortisol reactivity (assessed using multiple stressors).

Internalizing behaviours in relation to cortisol reactivity across multiple stressors. Laurent and colleagues (2015) conducted the first study to assess the association between internalizing behaviours, specifically, maternal-reported anxiety symptoms, and cortisol reactivity measured across multiple stressors. In addition to expanding the literature by employing two distinct stressor paradigms, this study included a middle-childhood sample. Children participated in both the TSST-C and a problem-solving stressor when they were between 9 and 10 years old. Generalized anxiety was associated with elevated cortisol in both challenges, specific phobia was associated with elevated cortisol levels in the TSST-C, and OCD was associated with prolonged cortisol (i.e., limited recovery) following a problem-solving stressor. Although this study examined how anxiety symptoms moderated cortisol reactivity across two, differentially potent, stressor paradigms, the findings do not indicate that children with lower anxiety symptoms demonstrate a more flexible cortisol response (i.e., higher cortisol levels in the more-potent challenge and lower levels in the less-potent challenge). This may be related to the nature of the stressors (i.e., the conflict discussion task may have been similarly potent as the TSST-C) or the specific internalizing moderator (anxiety) chosen. Nonetheless, Laurent et al. (2015) demonstrate the nuances in the associations between anxiety and cortisol reactivity, emphasizing the importance of using multiple stressor paradigms to assess these associations.

Laurent and colleagues (2016) conducted a second study to assess the differential relations between internalizing and externalizing behaviour problems and cortisol reactivity in response to two different stressors (Laurent, Vergara-Lopez, \& Stroud, 2016). Youth (age 8 to 17) participated in both a performance (including public speaking, mental arithmetic, and mirror tracing components) and 
interpersonal (peer rejection) stressor paradigm. Findings suggest the overall maternal-reported internalizing behaviours were not associated with cortisol reactivity in the performance challenge; however internalizing behaviours predicted an earlier cortisol peak (more negative linear term) and a less dynamic (rapidly changing) overall response curve (more positive quadratic term) in the interpersonal stressor. Further exploration of the components of internalizing behaviour suggests that anxious and withdrawal components of internalizing behaviour were driving the effect on cortisol reactivity in the interpersonal stressor. In addition, although global internalizing behaviours were not predictive of cortisol reactivity in the performance challenge, anxiety-specific symptoms were related to high cortisol levels during this challenge. Further, for females only, anxiety was associated with a later cortisol peak and more dynamic response curve during the performance challenge (in direct contrast to associations in the interpersonal stressor). These findings suggest that the associations between the specific type of internalizing behaviour measured and cortisol reactivity differ depending on gender and depending on the nature of the stressor paradigm. Further research is required to examine associations between internalizing behaviours and cortisol reactivity in multiple, and distinct, challenges, as well as to assess other potential moderators (e.g., gender) of these associations.

Differences in parent-reported and child-reported internalizing behaviours. In addition to the stressor paradigm used, another important factor that convolutes the association between internalizing behaviours and cortisol levels is the rater (i.e., parent or self) of internalizing behaviours. The low correlation (typically falling in the $.20 \mathrm{~s}$ ) between parent and child/adolescent reports of psychopathology is noted as one of the most robust findings in the developmental psychopathology literature (Achenbach \& Rescorla, 2001; De Los Reyes \& Kazdin, 2005). Overall, correlations between parents and children are low to moderate in magnitude, with lower correlations for internalizing problems compared to externalizing problems (Kazdin, 1994). Internalizing difficulties, specifically, 
are endorsed more by youth than their parents (Carlson, Kashani, Thomas, Vaidya, \& Daniel, 1987; Zahn-Waxler, Klimes-Dougan, \& Slattery, 2000), likely because of the internal and private nature of these difficulties. Despite the discrepancy between parent and child reports, both are thought to represent a valid, though unique, perspective on child difficulties. Taken together, this research suggests that given the unique predictive utility, reports from children and parents should be collected when examining internalizing behaviours.

Specifically, in relation to cortisol reactivity, studies that use adolescent self-reported depression or internalizing scales have shown that these symptoms are often associated with blunted cortisol reactivity (e.g., Janssens et al., 2012; Spies et al., 2011). Studies that use parent-reports to assess internalizing behaviours (i.e., using the CBCL; Achenbach, 2001) in samples of children and adolescents, have not found significant associations with cortisol reactivity (Attwood et al., 2011; Koss et al., 2014; Spinrad et al., 2009). To date, one study has separately assessed parent- and child-reported difficulties in relation to cortisol reactivity. Hartman, Hermanns, de Jong, and Ormel (2013) examined parent and adolescent reported internalizing behaviour in association with cortisol reactivity in a socialevaluative stressor (very similar to the TSST-C). Adolescent-reported, but not parent-reported, internalizing behaviour predicted cortisol responses. These findings suggest that the relation between cortisol reactivity and internalizing problems is likely impacted by the rater of such difficulties. However, given that only one study has directly examined this possibility, future research is needed to understand the potential impact of different raters on these associations.

Taken together, the literature to date demonstrates that internalizing problems are associated with cortisol levels, though these associations differ depending on the stressor paradigm and reporter of internalizing behaviours. Given the paucity of this literature and the methodological differences across studies (see Table 1), it is difficult to directly compare findings while taking into account these 
potential moderators. For example, only one study to date (Hartman et al., 2013) has included multiple informants of internalizing behaviours. In addition, only two studies to date (Laurent et al., 2015; Laurent et al., 2016) have examined associations between internalizing behaviours and intra-individual cortisol flexibility, assessed across multiple stressors. In addition to these potential conceptual and methodological factors related to the associations between internalizing behaviours and cortisol reactivity, there is physiological evidence that suggests that SAM activity, marked by sAA, may also be linked to these associations.

Internalizing behaviours and HPA and SAM coordination. Recent research suggests that sAA is associated with child and adolescent internalizing behaviours (e.g., Allwood et al., 2011; HillSoderlund et al., 2015). Given that activation of the HPA axis and SAM system are coordinated, the relations amongst cortisol, sAA, and internalizing behaviours are important to understanding atypical stress responsivity. Research has begun to examine associations among cortisol, sAA, and internalizing behaviours; however results are highly ambiguous and studies vary in terms of the type of stressor paradigm used, the rater (parent or child) of internalizing behaviours, and the specific outcome of interest (i.e., cortisol or internalizing behaviour).

Using an amalgamation of several different stressors (including cognitive, interpersonal conflict, and frustration activities), El-Sheikh et al. (2008) found that sAA moderated the impact of cortisol in predicting child internalizing behaviours, such that children who had both higher cortisol levels and higher sAA levels experienced greater internalizing behaviours. These findings are in line with Baurer et al.’s (2002) synchronous additive model of coordination and Del Giudice et al.'s (2011) vigilant pattern of coordination, both indicative of a maladaptive coordination pattern. Koss et al. (2015) examined how cortisol and sAA reactivity in response to a conflict vignette jointly predict child internalizing behaviours in the context of high/low family conflict. The results of this study suggest that 
children in a high conflict environment who evince low cortisol and high sAA levels experience the highest degree of internalizing behaviours. Allwood et al. (2011) also demonstrated that cortisol and sAA responses, to either a performance or peer-rejection stressor, interacted to predict child/adolescent behavior, but the nature of this interaction differed from other findings. Allwood et al. (2011) showed that individuals with high cortisol reactivity and low sAA reactivity had the highest behaviour problems (indicated by highest total score on the $\mathrm{CBCL}$ ). Further, it is important to highlight that these findings may differ because these studies examined different outcomes, that is, El-Sheikh et al. and Koss et al. assessed internalizing-specific outcomes, whereas, Allwood et al. assessed a combination of internalizing and externalizing difficulties. The mismatch in cortisol and sAA coordination found by these three studies is consistent with the asynchronous interactive model of coordination (Baurer et al., 2002).

In the three aforementioned studies, internalizing behaviour was the outcome of interest. In contrast, Bae et al. (2015) was interested in how internalizing behaviours interacted with sAA to predict cortisol reactivity during the TSST-C, in a sample of 8-14 year olds. Bae et al. found that higher internalizing behaviours coupled with a steeper sAA decline predicted lower cortisol during the TSSTC. In contrast to the aforementioned studies, additional studies have not found significant associations between cortisol, sAA, and internalizing behaviours (Chen et al., 2015; Vigil et al., 2010). As discussed above, challenges vary in their potency to elicit cortisol and sAA responses, a particularly important potential confound in the context of the present study, which addresses cortisol-sAA coordination. In addition, of course, the informant of internalizing behaviours in this research is also likely an important factor. However, the few aforementioned studies that examine the association between physiological coordination and internalizing problems all used parent-reported internalizing behaviours. Thus, it remains unclear whether the informant of internalizing behaviours impacts 
associations with physiological coordination. The specific associations between the child-reported and parent-reported internalizing problems on physiological coordination, will be directly assessed in the present study.

It is important to note that all of the aforementioned research is correlational in nature, and thus causal associations between internalizing behaviours, sAA, and cortisol have not been established. I highlight this point because the present study, similar to that of Bae et al. (2015), explores cortisol reactivity as an outcome, given our aim is to better understand factors that predict cortisol reactivity. As demonstrated by Bae et al., one system (e.g., SAM) can interact with psychological variables (i.e., internalizing behaviour) to predict functioning in another system (e.g., HPA). To this end, the interaction between other psychological variables (i.e., maltreatment status) and sAA has been shown to predict cortisol levels (Gordis et al., 2008). As such, I will explore the interaction between internalizing behaviours and sAA in the prediction of cortisol reactivity.

Taken together, the HPA literature demonstrates that 1) it is advantageous to assess cortisol reactivity across multiple contexts, given that situational stressors differ in the degree to which they elicit cortisol reactivity and flexibility across these challenges is an important metric in itself; 2) adaptive cortisol reactivity depends on coordination with the SAM system; and 3) individual differences in internalizing behaviours are likely related the aforementioned associations. However, research that simultaneously examines the associations between cortisol, sAA, and internalizing behaviours is limited and highly ambiguous. Findings may vary based on type of paradigm used to elicit stress as well as the reporter of internalizing behaviours. Given that the features of these studies vary so widely, it is difficult to determine consistent patterns across studies. In addition, much of this research is conducted with samples of adolescents; there is a dearth of literature examining these associations in middle-childhood. Given the impact of puberty on HPA activity (Stroud et al., 2011), it 
is important to examine these associations in middle childhood. Based on the above literature review, it is apparent that cortisol findings are confounded by a number of factors, including the stressor paradigm used to elicit a given stress response, coordination between cortisol and sAA activity, and the rater (parent or child) of internalizing behaviours.

\section{The Current Study}

Overall, this study aims to improve on the extant literature by 1) elucidating associations between internalizing behaviours and cortisol reactivity, and 2) assessing associations between internalizing behaviours and cortisol-sAA coordination, in a sample of 8-10 year-old children. I do so by employing several methodological controls, namely 1) utilizing two challenges, specifically designed to differentially elicit cortisol and sAA reactivity; 2) assessing sAA levels in coordination with cortisol levels in both of these challenges; and 3) using parent and child reports of internalizing behaviours. As such, this study is specifically designed to improve upon the methodological shortcomings in the extant literature. In doing so, this will be the first study to assess internalizing behaviours as they relate to both cortisol reactivity as well as coordination between cortisol and sAA, in two differentially potent challenges. In accordance with these study aims, I propose two hypotheses:

Hypothesis 1: I expect that internalizing behaviours will have distinct associations with cortisol reactivity in the two, differentially potent challenges. In particular, children with lower levels of internalizing behaviours will mount high cortisol reactivity in the potent TSST-C and minimal reactivity in the non-potent challenge. In contrast, children with higher levels of internalizing behaviours will demonstrate blunted cortisol reactivity in the TSST-C and either heightened or blunted reactivity in the non-potent challenge. To be clear, I will not statistically test whether internalizing problems are differentially predictive of cortisol responses across these two challenges (i.e., flexibility) for reasons discussed below, however, our methodological design 
permits the examination of associations amongst internalizing behaviours and cortisol reactivity, within each of these respective challenges.

Hypothesis 2: Internalizing behaviours are associated with coordination between salivary alpha-amylase and cortisol reactivity in both challenges. Given prior research (e.g., Bae et al., 2015), I expect that in the potent stressor, higher internalizing behaviour problems will be associated with lower cortisol and lower sAA, and in the non-potent challenge, higher internalizing behaviour problems will be associated with higher cortisol and higher sAA. However, given prior inconsistent findings (i.e., coordination in the positive and negative direction) the specific directions of these hypotheses are tentative. 


\section{Methods}

\section{Participants}

A total of 52 community-dwelling mother-child dyads, who are part of our laboratory's longitudinal study $(\mathrm{N}=297$ ) (Atkinson et al., 2013), participated in the current study. Families whose children ranged in age from 8.5 to 10 years old were contacted to participate in the present study. During the recruitment period, a total of 114 families had children between the ages of 8.5 and 10 years old, and thus were contacted to participate. Of these 114 families, I was unable to contact 42 families (because of change in contact information), 5 moved out of the Greater Toronto Area (GTA), 11 were interested but their schedules did not permit participation during the study period, and 4 were not interested in participating. The current sample did not differ from the larger longitudinal sample by sex $(t(330)=0.57, p=.57)$, ethnicity $(t(322)=0.96, p=.34)$, maternal education $(t(330)=-.31, p=.76)$, or income $(t(237)=-1.15, p=.25)$. Therefore, to my knowledge the individuals who participated in the present study did not differ on important demographic variables compared to the larger study sample. The larger study recruited mother-child dyads from postings and in-person visits at community centers as well as baby show conventions in the GTA. Inclusion criteria at the onset of this longitudinal study were that infants were healthy with no major developmental disorder, pregnancy was over 32 weeks; mothers were 18 years or older at childbirth, had no known hormonal disorders, and were fluent in English.

Children in this sample ranged from 8.5 to 10 years of age $(M=8.88, S D=0.70)$. The samples included 29 male children (55.7\%). The majority of the sample was Caucasian (76.5\%), with a smaller proportion of Asian (7.8\%), East-Indian (2.0\%), Afro-Canadian (2.0\%) and 'mixed' ethnicities (11.8\%). The sample was primarily from middle to high socio-economic backgrounds (69.5\% family income $>\$ 100,000)$. All demographic information was collected during the current study sessions in 
order to account of possible changes over time, given that this longitudinal research has spanned almost one decade. Of the 52 children who participated in the study, 46 completed both study sessions; five families could not be reached to schedule the second session, and one mother declined participation in the second session, expressing concern that study procedures were too stressful for the child. In addition, one child was excluded from the sample due to a diagnosis of Autism Spectrum Disorder (ASD), which prevented standard implementation of study procedures. Two other children had a reported 'high-functioning' ASD diagnosis but were able to complete standard study procedures ${ }^{1}$. In total, the final sample included 51 children, 45 of whom completed both study sessions. All 51 children were included in the analyses, given that multi-level modeling allows for missing data (explained further below).

\section{Procedure}

Mother-child dyads participated in two laboratory-based study sessions, approximately three weeks apart (Median $=2.00$ weeks, Interquartile Range $=2.00$ weeks, range 1 day-12 weeks), at Ryerson University. Each study session lasted two hours. At the beginning of each session, the experimenter explained study procedures to children and mothers and obtained assent and consent, respectively. After the consent process, children and mothers relaxed for 15 minutes, during this time two baseline saliva samples were collected (-15 min and $0 \mathrm{~min})$. During this baseline period, dyads completed initial measures of positive and negative affect as well as a visual analog rating of momentary stress (baseline self-reported stress are commonly captured at the same time as physiological measures; e.g., Doom, Doyle, \& Gunnar, 2015). Immediately after the second saliva sample was taken (0 min), children participated in a 20-minute challenge. During one visit, children participated in a performance stressor, the child-appropriate version of the Trier Social Stress Test

\footnotetext{
${ }^{1}$ Results did not differ whether these two individuals were included in the sample or excluded. Thus, these individuals were retained in the sample and included in the subsequent analyses.
} 
(TSST-C; Buske-Kirschbaum et al., 1997). During the other visit, the children participated in a competition challenge, which included a timed puzzle task and an adapted mirror-tracing activity (Bostock, Hamer, Wawrzyniak, Mitchell, \& Steptoe, 2011; Hammer et al., 2006). The duration of each challenge was equivalent (20 minutes), in order to minimize differential timing effects of cortisol/sAA trajectory. After each challenge, the experimenter gave children positive feedback about their performance. The order of the challenges was randomly determined in order to achieve counterbalancing ( $49 \%$ of the sample participated in the TSST-C during the first session). Following these challenges, mothers and children completed questionnaires (including a demographic questionnaire, the Child Depression Inventory (CDI), and the Child Behavior Checklist (CBCL)). In total, saliva was sampled before ( -15 and $0 \mathrm{~min})$ and after $(+5,+10,+20,+40$, and $+60 \mathrm{~min})$ each challenge. During the first study session, children were weighed and their height was measured to later calculate their Body Mass Index (BMI) (calculated by the weight in kg divided by the square of the height in metres (i.e., $\mathrm{kg} / \mathrm{m}^{2}$ ); World Health Organization, 2017) as a covariate of stress reactivity. Procedures occurred between $1300 \mathrm{~h}$ and $1800 \mathrm{~h}$ to control for circadian effects on cortisol and sAA. This time frame is based on my review of the typical start and end times used in studies that examine cortisol in response to laboratory stress (e.g., Bostock, Hamer, Wawrzyniak Mitchell, \& Steptoe, 2011; Chopra et al., 2009).

\section{Measures}

The Trier Social Stress Test- Child Version (TSST-C). The TSST (Kirschbaum, Pirke, \& Hellhammer, 1993) is an uncontrollable, social-evaluative, performance challenge, which has been adapted for children 7 years and older (TSST-C; Buske-Kirschbaum et al., 1997). The TSST-C consisted of a 5-minute instructional period, 5-minute preparation period, 5-minute speech, and 5minute arithmetic task (20 minutes total). The TSST-C began with the experimenter reading the child 
the beginning of a story (see Appendix 1) in front of two expert judges, the child was then given time to prepare the rest of the story in a separate room, thereafter the child finished telling the story as excitingly as possible in front of two expert judges. If a child finished their story before five minutes elapsed or did not speak for 20 seconds, one of the judges prompted them that there was time remaining and asked them to continue. After completing the story portion of the task, the judge asked the child to serially subtract the number 7 from 758 as quickly as possible, without making mistakes. During the arithmetic task, if the child made a mistake the judge instructed them to stop and start the task over again. Children were informed that the task would be video-recorded and later reviewed by the expert judges. Two confederates in laboratory coats acted as expert judges and displayed a neutral expression throughout the challenge. The TSST has been shown to reliably elicit cortisol responses in both children and adults (Buske-Kirschbaum et al., 1997; Dickerson \& Kemeny, 2004; Kirschbaum et al., 1993; Kirschbaum \& Hellhammer, 1994; Yim, Quas, Cahill, \& Hayakawa, 2010).

Competition Challenge. Children completed a competition challenge, which consisted of a 5minute instruction period, 5-minute mirror tracing task (Bostock et al., 2011; Hammer et al., 2006) and a 10-minute puzzle activity. At the beginning of this challenge, children were told they are going to participate in a competition and that their performance will be compared to that of the other children in the study, so they should try to perform their best by doing the tasks as quickly as possible without making mistakes. First, children completed the adapted (described below) 5-minute mirror tracing activity (Bostock et al., 2011; Hammer et al., 2006). During this activity, a piece of paper with the outline of a star shape was placed underneath a mirror tracing apparatus (see Appendix 2), such that children could only see the reflection of the star through a mirror. Children were asked to trace the outline of a star by looking at the reflection in a mirror. They were told to try their best to trace the star as quickly as they can, without making mistakes (i.e., tracing over the lines of the star). Children were 
told they should trace another star when they are finished tracing the first. Unlike the original mirrortracing task (Bostock et al., 2011; Hammer et al., 2006), this task was adapted so that the apparatus did not buzz when children made a mistake. This feedback was not included in this task to prevent interference with the amount of effort put forth in the challenge. The second part of the competition was a 100-piece puzzle to be completed within 10-minute time limit (see Appendix 3). The puzzle was designed to be too complex to complete within this time frame. Children were asked to work as quickly as they could to complete the puzzle. They were instructed that if they finished the puzzle they would be given another one to complete. Children were told that the child who finishes the most puzzles wins the competition. None of the children completed the puzzle in the time provided.

Ethical considerations. The aforementioned challenges are deemed to be no more stressful than stressors encountered in everyday life (Buske-Kirschbaum et al., 1997; Kirschbaum et al., 1993; Zilioli \& Watson, 2012). If the child became noticeably upset during either of the challenges, the confederate judges or experimenter asked them if they were okay to continue. The experimenters emphasized that the children were free to stop participation at any time, without penalty. Mothers observed all procedures and were also free to terminate at any time should they wish. In no case did mothers terminate the challenge prematurely. A total of ten and four children prematurely terminated the TSST$\mathrm{C}$ and competition challenge, respectively (only one child terminated both challenges prematurely) ${ }^{2}$. Immediately after all challenges, the experimenter provided positive performance feedback to the child. In addition, after the TSST-C, the experimenter explained that the judge's flat affect was not a reflection of their performance, but rather was a tactic to make the challenge more stressful. Further, at the end of this session (i.e., after the last saliva sample is taken) children were offered an opportunity to

\footnotetext{
${ }^{2}$ Children who terminated the TSST-C or competition challenge prematurely did not differ in their cortisol levels from those who completed the challenges $[\mathrm{t}(267)=-1.43, p=.15$ and $\mathrm{t}(304)=1.26, p=.21$, for TSST-C and competition challenge, respectively].
} 
meet the expert judges so that they could receive positive verbal and non-verbal feedback. Complete disclosure was provided at the end of each study session. That is, during the session with the TSST-C, children were told that the judges are actually university student volunteers and during the session with the competition, children were told that their performance on the competition would not actually be compared to that of other children in the study.

Saliva collection and assays. Acute HPA and SAM activity were measured by salivary cortisol and alpha amylase, respectively. To avoid contamination, participants were asked to refrain from drinking, eating, or brushing their teeth for one hour before saliva sampling (Kirschbaum \& Hellhammer, 1994, 1989). In response to an intake inventory, all participants reported having complied with these requests, however, one child sipped water during the session due to hiccups. Saliva was collected from children, using sorbettes (Salimetrics, State Collee PA). Saliva was collected seven time points throughout both study sessions $(-15,0,+5,+10,+20,+40$, and $+60 \mathrm{~min})$, to sufficiently measure baseline, reactivity and recovery (Dickerson \& Kemeny, 2004; Yim et al., 2010). Cortisol was assayed using the $-15,0,+10,+20,+40$, and +60 min samples, whereas sAA was assayed using the $-15,0,+5$, +10 , and +20 min samples, given that cortisol peaks between +20 and +40 min and sAA peaks between +5 and +10 min (Dickerson \& Kemeny, 2004; Nater \& Rohleder, 2009; Villani, in prep). After collection, sorbettes were placed in a $2-\mathrm{mL}$ cryovial, sealed, and stored at $-70^{\circ} \mathrm{C}$. Samples were thawed overnight in the refrigerator before they were assayed. Saliva samples were centrifuged for $20 \mathrm{~min}$ at $3000 \mathrm{rpm}$ at $4{ }^{\circ} \mathrm{C}$ for cortisol assays and for $15 \mathrm{~min}$ at $3000 \mathrm{rpm}$ at $4{ }^{\circ} \mathrm{C}$ for alpha-amylase assays. Saliva was assayed using cortisol and alpha-amylase immunoassay kit (Salimetrics, State College, PA). Samples from each child across both sessions were assayed together. All samples were assayed in duplicate and average values were used in analyses. For cortisol, the inter-assay variation 
was $7.96 \%$ and the intra-assay variation was $8.45 \%$. For sAA, the inter-assay variation was $9.43 \%$ and the intra-assay variation was below 5\% for low, medium, and high samples.

Questionnaires. Children and their mothers completed several questionnaires following the challenge in the first study session.

Child-reported depressive symptoms. Child self-reported depressive symptoms were assessed using the 27-item Child Depression Inventory, Second Edition (CDI-2; Kovacs, 1992). The CDI-2 is a comprehensive measure of depressive symptoms for children and adolescents 7 to 17 years old. The CDI-2 yields a total score of depressive symptoms within the past two-weeks, as well as four subscales: negative mood, negative self-esteem, ineffectiveness, and interpersonal problems. The CDI is a psychometrically sound measure, with high reliability and predictive validity in both community and clinical samples of children and adolescents (Masip, Amador-Campos, Gómez-Benito, \& Gándara, 2010).

Maternal reported internalizing behaviours. Given that parent and child-reported internalizing difficulties have been shown to be differentially associated with cortisol outcomes (e.g., Attwood et al., 2011; Spies et al., 2011) and that using multiple informants is considered best practice when assessing child and youth psychopathology (Achenbach, 2009; Jensen et al., 1999; Lahey et al., 1996), mothers completed the parent-version of the Child Behavior Check List for school-aged children, 6-18 years old (CBCL 6-18; Achenbach, 2001). The CBCL is one of the most frequently used and empirically validated measures of child psychopathology. The CBCL has been validated in numerous clinical and community samples, worldwide (e.g., Breland-Noble \& Weller, 2012; Malik, 2008). The instrument produces eight syndrome scales (Withdrawn, Somatic Complaints, Anxious/Depressed, Social Problems, Thought Problems, Attention Problems, Delinquent Behavior and Aggressive Behavior) as well as a Total Problem Score and two broad subscales, the Internalizing and Externalizing scales. Of 
importance for the current study, the Internalizing scale consists of the Withdrawn, Somatic Complaints and Anxious/Depressed syndrome subscales.

Demographics and covariates. Demographic information, including the age, sex, BMI, and ethnicity of children, as well as family income were examined as potential covariates. In addition, the time gap between sessions, and sleep and waking times were assessed as covariates, as these variables can potentially influence HPA and sAA trajectories (e.g., Goldberg et al., 2003; Ruttle, Javaras, Klein, Armstrong, Burk, \& Essex, 2013; Stadler et al., 2012).

\section{Data Preparation and Analytic Approach}

Data were analyzed using multilevel modelling (MLM) with maximum likelihood (Hierarchical Linear Modeling, Version 7, Raudenbush, Bryk, Cheong, Congdon, du Toit, 2011) to account for interdependence of cortisol and sAA samples across time and within individual participants. MLM is ideal for physiological (i.e., cortisol) analyses because it accounts for different initial values of the dependent variable (i.e., intercept) and different rates of change within and across individuals (growth trajectories, i.e., slopes) and it permits the examination of how predictors of interest influence these associations (Hruschka, Kohrt, \& Wrothman, 2005; Singer \& Willet, 2003; Willett \& Sayer, 1994). Rather than employing several linear regression analyses to assess these associations, MLM permits refined examinations of trajectories in a single analysis. The use of trajectories are advantageous in comparison to summary physiological indices, such as area under the curve with respect to change $\left(\mathrm{AUC}_{\mathrm{I}}\right)$, which can be considered crude measurements due to the loss of intra-individual variability across physiological assessment time points (Hruschka et al., 2005). In addition, MLM derives parameter estimates by employing a full information maximum likelihood (FIML) technique, thus allowing for data to be missing at Level 1 (in this case, cortisol and sAA) (Hruschka et al., 2005; Hibel et al., 2009; Laurent et al., 2015). Further, MLM does not require data 
points to be evenly spaced in time, thus permitting analyses of cortisol and sAA collected at different time points (Atkinson et al., 2013).

A two-level multi-level model was used to assess child sAA and cortisol trajectories across collection time (i.e., -15, 0, 5, 10, 20, 30, 40, and 60 min; Level 1) nested within individual children (Level 2). Four separate multi-level models were conducted to assess associations in both challenges (i.e., TSST-C and puzzle/mirror-tracing competition) and both psychological predictors (i.e., childreported depressive symptoms and maternal reported internalizing behaviours). A total of four models was tested (1. TSST-C and child-reported depressive symptoms, 2. TSST-C and maternal reported internalizing behaviours; 3 . Competition challenge and child-reported depressive symptoms; 4 . Competition challenge and maternal reported internalizing behaviours). Intra-class correlations (ICC) depicting the variance accounted for between levels support the use of nesting procedures. For the null models the ICCs were .56 and .64 for the TSST-C and competition challenge, respectively. The ICC represents the percentage of variance between units at different levels; thus, $36 \%$ to $44 \%$ of the variance in cortisol was between time and individual.

For all models, cortisol was inputted as the outcome variable. Level 1 predictors included sAA (group mean centered), linear time (coded as -1, 0, 1, 2, 3, 4, corresponding to the $-15,0,10,20,40$, and $60 \mathrm{~min}$; uncentered) and quadratic time (coded as 3.3, -0.6, -2.6, -2.6, -0.6, 3.3, corresponding to the $-15,0,10,20,40$, and $60 \mathrm{~min}$; uncentered). In order to minimize multi-colinearity between linear time and quadratic time, orthogonal polynomial contrasts were used to represent quadratic time (Little, 2013; Raudenbush et al., 1995). Given its relevance for understanding the structure of the data, regardless of significance, linear time was retained in all models. In addition to sAA, linear time, and quadratic time as main effects, interaction terms between sAA and each time variable (i.e., sAA x linear time, sAA x quadratic time) were also entered as Level 1 predictors. At Level 2, the relevant 
internalizing predictor (either child-reported or maternal-reported) was entered (grand mean centered). In addition, at Level 2, several two-way and three-way interactions between the Level 2 internalizing predictor and Level 1 predictors were entered (i.e., linear time x predictor, quadratic time x predictor, sAA x predictor, linear time $\mathrm{x}$ sAA $\mathrm{x}$ predictor, quadratic time $\mathrm{x}$ sAA $\mathrm{x}$ predictor).

A multi-step, bottom-up approach, using the full maximum likelihood estimation procedure was used to derive the final model. Theoretical considerations, significance testing, a comparison of model fit using the log-likelihood ratio test, and an estimate of variance explained (for fixed effects) were used to determine final model components, including fixed and random effects (Raudenbush \& Byrk, 2002). The random effects (slopes) for linear time, quadratic time, and sAA were tested at Level 1. Final estimation without robust standard errors (given the small sample size) was used to interpret fixed effects. Once a parsimonious model was established, the influence of potential covariates was assessed and if significant, the covariates were retained in the final model. All final models were reestimated using restricted maximum likelihood for a conservative estimation of variance-covariance structure. The equations and results corresponding to each step of this bottom up model-building approach are included in Appendix 4. The equations and results from the final models are reported below. 


\section{Results}

\section{Descriptive Results and Exploring Covariates}

Descriptive statistics (i.e., means, standard deviations, and ranges) for untransformed and logtransformed cortisol and sAA, child-reported depressive symptoms, and maternal-reported internalizing problems are presented in Table 3. Cortisol and sAA values were log transformed to address positive skew. Child self-reported depressive symptoms scores ranged from 0 to $30(M=7.57, S D=6.15)$. In total, $12 \%$ of the sample reported CDI scores in the Clinical range, $8 \%$ reported scores in the Borderline Clinical range, $80 \%$ reported scores in the Non-Clinical range. Maternal-reported internalizing scores ranged from 0 to $22(M=8.00, S D=5.81)$. In total, $23 \%$ of the sample reported internalizing scores in the Clinical range, $12 \%$ reported scores in the Borderline Clinical range, $65 \%$ reported scores in the Non-Clinical range. Correlations between cortisol, sAA, child-reported depressive symptoms, and maternal reported internalizing problems are shown in Table 4. 
Table 3. Descriptive statistics.

$\begin{array}{lll}\text { M } & \text { SD } & \text { Range }\end{array}$

Cortisol TSST-C (untransformed) $-15 \mathrm{~min}$

4.2703

3.59971

$0.7-19.07$

$0 \mathrm{~min}$

4.2937

4.15839

1.04-24.49

$10 \mathrm{~min}$

9.3536

16.48566

$1.14-86.25$

$20 \mathrm{~min}$

8.2945

13.63331

$1.36-73.58$

$40 \mathrm{~min}$

5.5243

6.70677

$1.46-35.27$

$60 \mathrm{~min}$

4.3026

3.19565

$1.41-18.98$

sAA TSST-C (untransformed)

$\begin{array}{rrrr}-15 \mathrm{~min} & 68.8185 & 43.61579 & 9.84-205.66 \\ 0 \mathrm{~min} & 67.066 & 42.4926 & 8.12-190.90 \\ 5 \mathrm{~min} & 76.7814 & 49.9943 & 10.82-198.44 \\ 10 \mathrm{~min} & 65.292 & 44.00245 & 8.69-238.46 \\ 20 \mathrm{~min} & 60.09 & 32.82355 & 10.33-147.93\end{array}$

Cortisol COMP (untransformed)

$\begin{array}{rrrr}-15 \mathrm{~min} & 3.4412 & 2.1291 & 0.43-12.55 \\ 0 \mathrm{~min} & 3.1376 & 2.07347 & 0.68-14.46 \\ 10 \mathrm{~min} & 2.9078 & 1.92033 & 0.39-11.76 \\ 20 \mathrm{~min} & 2.8236 & 1.90579 & 0.37-10.93 \\ 40 \mathrm{~min} & 2.9998 & 1.78156 & 0.62-12.3 \\ 60 \mathrm{~min} & 3.658 & 2.79261 & 0.52-17.53\end{array}$

sAA COMP (untransformed)

$\begin{array}{rrrr}-15 \mathrm{~min} & 71.9889 & 45.04542 & 16.56-239.44 \\ 0 \mathrm{~min} & 70.3159 & 38.27412 & 10-167.77 \\ 5 \mathrm{~min} & 64.6422 & 29.9924 & 8.69-136.78 \\ 10 \mathrm{~min} & 63.8885 & 36.60485 & 9.02-158.42 \\ 20 \mathrm{~min} & 64.9998 & 36.8897 & 9.59-152.36\end{array}$

CDI self-reported depression

7.57

6.146

0-30

CBCL maternal-reported

8.00

5.811

$0-22$

internalizing symptoms

Note: TSST $=$ Trier Social Stress Test- Child Version; COMP $=$ Competition challenge (Puzzle/mirror tracing activities); $\mathrm{sAA}=$ salivary alpha amylase $\mathrm{CDI}=$ Child Depression Inventory; $\mathrm{CBCL}=\mathrm{Child}$ Behaviour Checklist. 
Table 4. Correlations between salivary cortisol, salivary alpha amylase, and internalizing predictors.

\begin{tabular}{lllllll}
\hline & 1. & 2. & 3. & 4 & 5. & 6. \\
\hline 1. $\log$ Cortisol TSST & --- & & & & & \\
2. log Cortisol COMP & $.40^{* *}$ & --- & & & & \\
3. sAA TSST & -.10 & -.10 & --- & & & \\
4. sAA COMP & -.09 & $.20^{* *}$ & $.55^{* *}$ & --- & & \\
5. CDI & -.08 & -.06 & $.26^{* *}$ & $.19^{* *}$ & --- & \\
6. CBCL Internalizing & $-.16^{*}$ & -.04 & $.30^{* *}$ & .04 & .18 & ---
\end{tabular}

Note $:$ TSST $=$ Trier Social Stress Test; COMP $=$ Competition challenge (puzzle and mirror tracing activities); sAA = salivary alpha amylase (log transformed); CDI = Child Depression Inventory total score; $\mathrm{CBCL}=$ Child Behavior Checklist Internalizing subscale.

${ }^{*} p<.05, * * p<.01$ 
Several socio-demographic factors (i.e., child age, sex, BMI, ethnicity and family income), as well as wake and breakfast time, were assessed as potential covariates with child salivary cortisol (Table 4). In regard to child sex, females showed higher mean cortisol levels in the TSST-C compared to males $(t(249)=-2.62, p<.01)$, however, there were not significant sex-specific cortisol differences in the competition challenge $(t(297)=-0.62, p=.54)$. Child sex and wake and breakfast time were significantly correlated with salivary cortisol and thus were examined as potential covariates in subsequent MLM models. Potential covariates were included in the most parsimonious MLM model, however none of the potential covariates were significant, thus they were removed from the final MLM model. 
Table 5. Correlations between potential covariates and salivary cortisol.

\begin{tabular}{|c|c|c|c|c|c|c|c|c|c|}
\hline & 1. & 2. & 3. & 4. & 5. & 6. & 7. & 8. & 9. \\
\hline $\begin{array}{l}\text { 1. Log salivary } \\
\text { cortisol }\end{array}$ & --- & & & & & & & & \\
\hline 2. BMI & .01 & --- & & & & & & & \\
\hline 3. Sex & $.09 *$ & $.17^{* *}$ & --- & & & & & & \\
\hline 4. Age & -.03 & .04 & $-.23 * *$ & --- & & & & & \\
\hline 5. Ethnicity & $.09 *$ & -.08 & -.01 & $-.09 *$ & --- & & & & \\
\hline 6. Family income & .08 & .02 & $-.17 * *$ & -.002 & -.05 & --- & & & \\
\hline 7. Wake time & $.10^{*}$ & -.03 & .12 & .06 & -.07 & .18 & --- & & \\
\hline 8. Breakfast time & $.15 * *$ & .02 & .07 & .01 & -.004 & $.55 * *$ & .08 & --- & \\
\hline 9. Time (days) & $-.16 * *$ & -.11 & .09 & $-.29 * *$ & $-.24 * *$ & -.06 & -.09 & $-.11 * *$ & --- \\
\hline between lab visits & & & & & & & & & \\
\hline
\end{tabular}

Note: $\mathrm{BMI}=$ Body Mass Index

${ }^{*} p<.05, * * p<.01$ 


\section{Visual Inspection of Cortisol and Alpha Amylase Trajectories Across Challenges}

Salivary cortisol and sAA trajectories of all participants across time and challenge are depicted in Figure 5 Panel A and B, respectively. As shown in Panel A of Figure 5, in the TSST-C paradigm (grey dashed line), cortisol levels peak at approximately 20 minutes and then decline and returns to baseline by 60 minutes; in contrast, in the puzzle/mirror tracing competition (black solid line), cortisol levels decline from baseline to 20 minutes and then begin to increase. It is possible that the post- 20 minute increase is associated with the completion of study questionnaires (completed between 10 and 30 minutes post-challenge) or the post-challenge uncontrolled free time. Overall, this demonstrates that the TSST-C elicits a relatively strong cortisol response, whereas the competition challenge does not. As shown in Panel B, sAA appears to peak at 5 minutes and return to baseline by 20 minutes post-TSSTC; whereas, sAA declines continuously from baseline to 20 minutes post-competition challenge. Taken together, this visual inspection of cortisol and sAA values suggests that across the entire sample as a whole, the TSST-C elicits both cortisol and sAA reactivity, whereas cortisol and sAA decline across time in the competition challenge. 


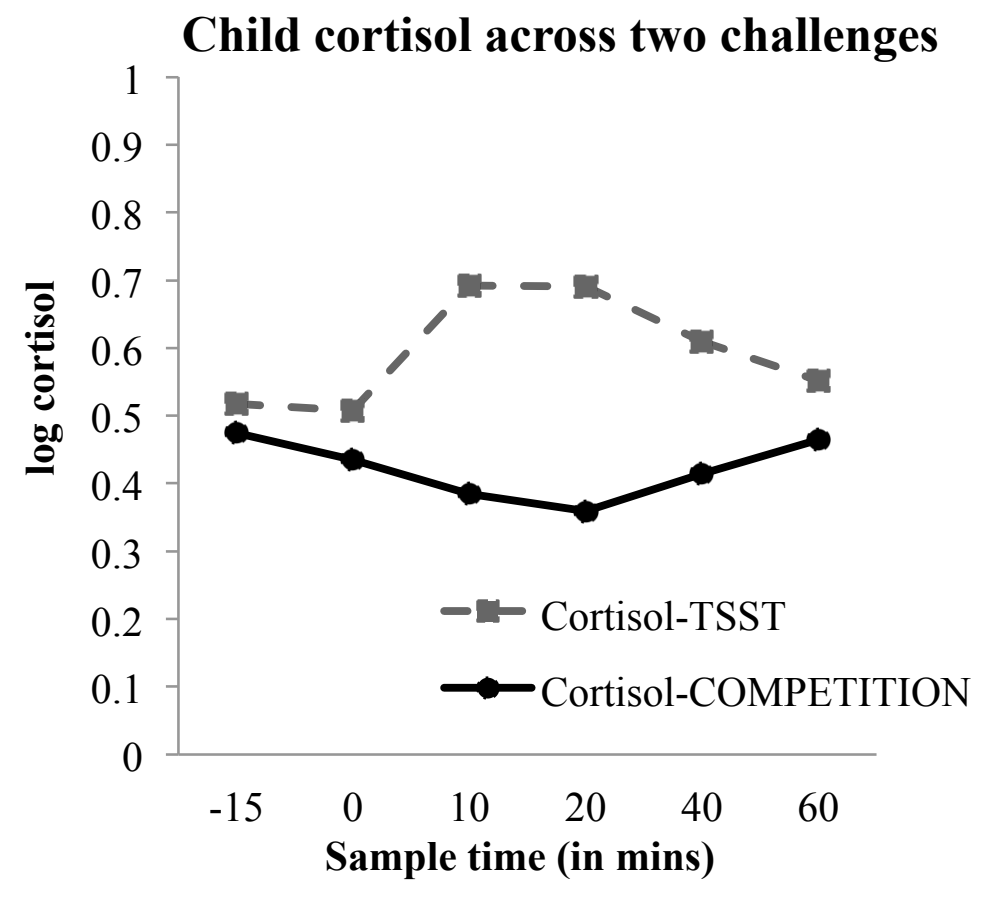

A

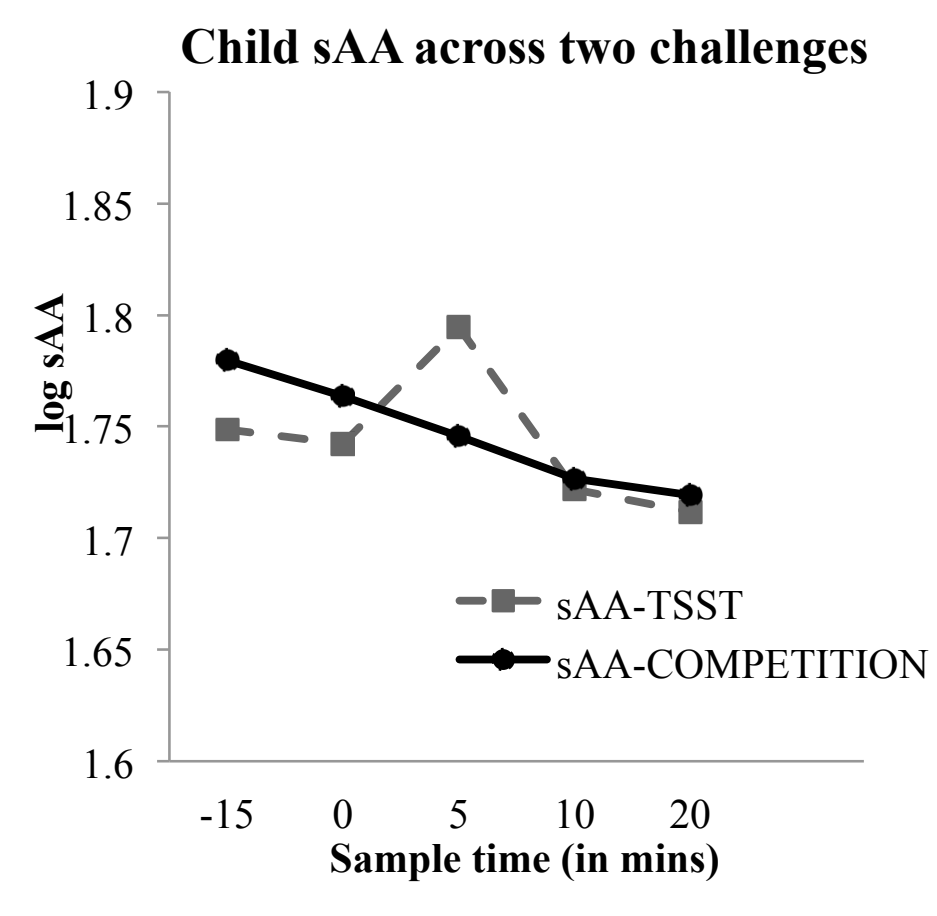

B

Figure 5. Panel A depicts salivary cortisol trajectories across the Trier Social Stress Test (TSST) and puzzle/mirror challenge (COMPETITION). Panel B depicts salivary alpha amylase (sAA) trajectories across the Trier Social Stress Test-Child Version (TSST-C) and puzzle/mirror challenge (COMPETITION). 


\section{Main Analyses}

Trier Social Stress Test-Child Version (TSST-C). Two separate models were used to examine the effect of child self-reported depressive symptoms and maternal-reported internalizing problems on cortisol trajectories in the TSST-C.

Child-reported depressive symptoms predicting cortisol in the TSST-C. The final model fit the data significantly better than the null model $\left(\chi^{2}(6)=52.95, p<.001\right)$. The final model (shown in equation 1) accounted for $61 \%$ of the variance between individuals. Model coefficients and standard errors of fixed effects are shown in Table 6. At Level 1, the fixed effect for time as a quadratic function significantly improved model fit. The random effect (slopes) for quadratic time was significant $\left(\chi^{2}(38)\right.$ $=98.37, p<.001)$ and significantly improved model fit, and thus slopes were allowed to vary across quadratic time in the model. The fixed effect of linear time was retained in the model, although it was not significant. The fixed effect of sAA as well as the interactions between sAA and linear and quadratic time were entered at Level 1, however they were not significant, and thus were ultimately removed after testing for potential higher level interactions (see Appendix 4.1). In addition, the random effects of linear time and sAA were tested, but because they were unreliable estimates, were not significant, and did not improve model fit, they were not retained in the model.

At Level 2, child-reported depressive symptoms (CDI), as well as the interactions between CDI and Level 1 predictors (i.e., CDI x sAA, CDI x linear time, CDI x quadratic time, CDI x linear time $\mathrm{x}$ sAA, CDI x quadratic time $\mathrm{x}$ sAA) were entered as fixed effects. The CDI $\mathrm{x}$ linear time and CDI $\mathrm{x}$ quadratic time interactions were significantly different from zero $(p<.05)$, indicating that the regression of cortisol on child depressive symptoms varies across time (see Figures 6 and 7). The addition of the CDI $x$ linear time and CDI x quadratic time interactions led to significantly improved model fit, $\chi^{2}(1)=13.02, p<.01$, and accounted for $31 \%$ additional variance. All other Level 2 
interactions were not significant (Appendix 4.1) and thus were removed from the final model.

Covariates (i.e., sex, ethnicity, wake and eating time) were also added at Level 2, but were not significant and did not significantly improve model fit, and thus were removed from the final model (Table 6).

\section{Equation 1:}

Level-1 Model

Log Cortisol TSST $-\mathrm{C}=\mathrm{B} 0+\mathrm{B} 1 *$ Linear Time $+\mathrm{B} 2 *$ Quadratic Time $+\mathrm{r}$

Level-2 Model

$$
\begin{aligned}
& \mathrm{B} 0=\mathrm{Y} 00+\mathrm{Y} 01 * \text { Child Depression }(\mathrm{CDI})+\mathrm{u} 0 \\
& \mathrm{~B} 1=\mathrm{Y} 10+\mathrm{Y} 11 * \mathrm{CDI} \\
& \mathrm{B} 2=\mathrm{Y} 20+\mathrm{Y} 21 * \mathrm{CDI}+\mathrm{u} 2
\end{aligned}
$$

Mixed Model

Log Cortisol TSST-C $=$ Y00 + Y01*CDI + Y10*Linear Time + Y11*CDI *Linear Time + Y20* Quadratic Time Y21*CDI* Quadratic Time $+\mathrm{u} 0+\mathrm{u} 2 *$ Quadratic Time $+\mathrm{r}$ 
Table 6. Fixed effect estimates from the final 2-level multilevel model with child-reported depression predicting log transformed cortisol levels in the Trier Social Stress Test.

\begin{tabular}{lccc}
\hline Fixed Effect & $\gamma$ & $S E$ & $t$ \\
\hline Intercept & .57 & .04 & $13.63^{* * *}$ \\
CDI & -.005 & .007 & -0.76 \\
Linear time & .008 & .007 & 1.20 \\
Quadratic time & -.02 & .007 & $-2.44^{*}$ \\
Linear time*CDI & -.003 & .001 & $-2.52^{*}$ \\
Quadratic time*CDI & .003 & .001 & $2.63^{*}$ \\
\hline Random Effect & Variance & Standard Deviation & Chi-Square (df) \\
& & & $493.87(38)^{* * *}$ \\
Intercept & .06 & .25 & $98.37(38)^{* * *}$ \\
Quadratic Time slope & .01 & .04 & \\
Level-1 & .03 & .17 & \\
\hline Note. CDI Child Depression & &
\end{tabular}

Note. CDI $=$ Child Depression Inventory.

${ }^{*} p<.05,{ }^{* *} p<.01,{ }^{* * *} p<.001$ 
In order to better understand the CDI x linear time interaction, a multilevel model interaction computation tool was used (Preacher, Curran, \& Bauer, 2006). Conditional values of the mean, as well as 1 standard deviation (SD) above and below the mean were selected for the child-reported depressive symptom predictor. All time points were included for linear sampling time $(-1,0,1,2,3,4)$. Simple slope analyses for the significant two-way interaction between linear time and CDI predicting salivary cortisol, demonstrated a marginally significant association between salivary cortisol and time at high levels of depressive symptoms $(\beta=-0.03, \mathrm{SE}=.02, z=1.82, p=.069)$, but not at moderate $(\beta=-0.01$, $\mathrm{SE}=.01, z=1.21, p=.23)$ or low levels of depressive symptoms $(\beta=0.004, \mathrm{SE}=.01, z=0.62, p=$ .53) (Figure 6). Similarly, the two-way interaction between CDI and quadratic time was plotted using the same conditional values of mean, as well as $1 \mathrm{SD}$ above and below the mean of the moderator variable (Aiken \& West, 1991; Dawson, 2014). This representation shows a similar effect, such that individuals with lower levels of depressive symptoms show an increase followed by a decline in cortisol over time, whereas individuals with higher levels of depressive symptoms show an initial decline followed by a slight increase in cortisol across time (Figure 7). 
Child Depressive Symptoms by Linear Time Interaction in the TSST

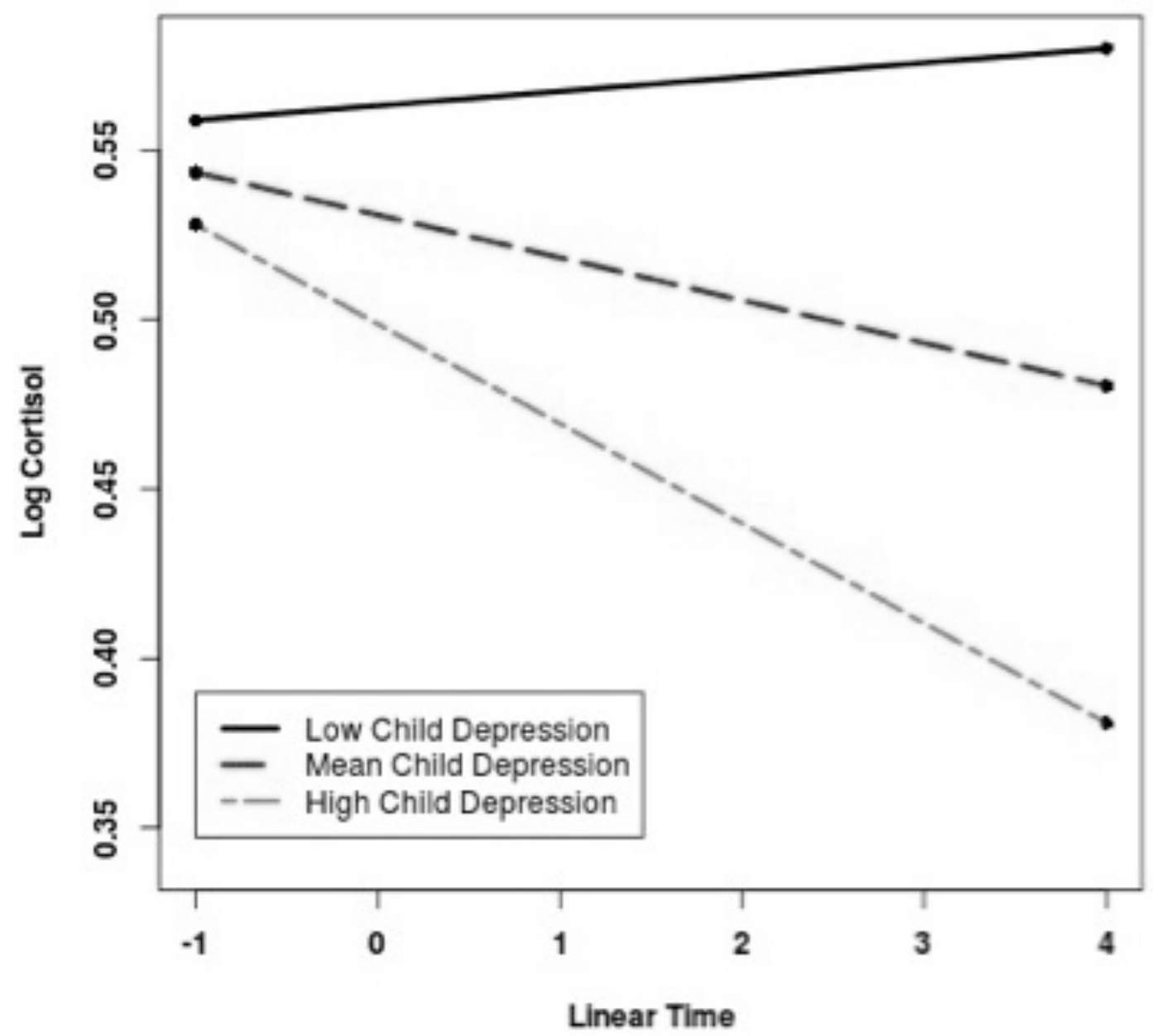

Figure 6. Interaction between self-reported child depressive symptoms and saliva sampling time (linear) predicting log transformed cortisol levels in the Trier Social Stress Test. 


\section{Child Depressive Symptoms by Quadratic Time Interaction TSST}

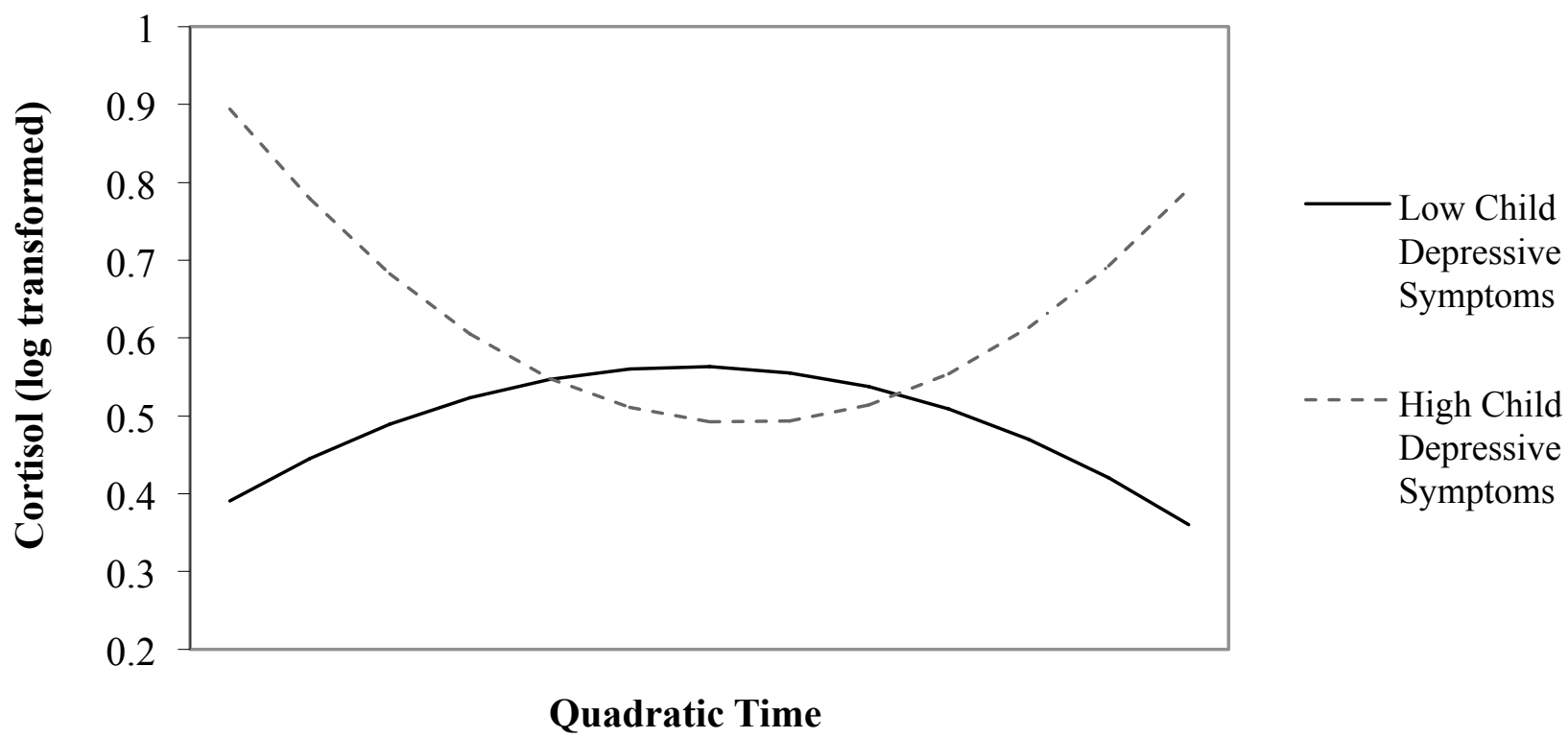

Figure 7. Interaction between self-reported child depressive symptoms and saliva sampling time (quadratic) predicting log transformed cortisol levels in the Trier Social Stress Test. 
Maternal-reported internalizing behaviours predicting cortisol in the TSST-C. The final model fit the data significantly better than the null model $\left(\chi^{2}(3)=22.71, p<.001\right)$. The final model (shown in equation 2) accounted for $66 \%$ of the variance between individuals. The predictor of interest, maternal-reported internalizing problems $(\mathrm{CBCL})$, was not significant as a main effect or interaction (e.g., CBCL x time or CBCL x sAA). At Level 1, the fixed effect for time as a quadratic function significantly improved model fit. The random effect (slopes) for quadratic time was significant $\left(\chi^{2}(39)\right.$ $=111.93, p<.001)$ and significantly improved model fit, and thus slopes were allowed to vary across quadratic time in the model. The fixed effect of linear time was retained in the model, although it was not significant. The fixed effect of sAA as well as the interactions between sAA and linear and quadratic time were also entered at Level 1, however they were not significant, and thus were ultimately removed after testing for potential higher level interactions (see Appendix 4.2). At level 2, maternal-reported internalizing problems (CBCL), as well as the interaction between CBCL and Level 1 predictors (i.e., CBCL x sAA, CBCL x linear time, CBCL x quadratic time, CBCL x linear time $\mathrm{x}$ sAA, CBCL x quadratic time x sAA) were entered as fixed effects (see Appendix 4.2). The maternal reported internalizing behaviours as well as all Level 2 interactions were not significantly different from zero and did not significantly improve model fit. All non-significant Level 1 and Level 2 effects were removed from the model. The final model (Equation 2) included linear and quadratic time as fixed effects, and quadratic time as a random effect (Table 7).

\section{Equation 2:}

Level-1 Model Log Cortisol TSST-C $=$ B0 + B1*Linear Time + B2*Quadratic Time $+r$

Level-2 Model

$$
\begin{aligned}
& \mathrm{B} 0=\mathrm{Y} 00+\mathrm{u} 0 \\
& \mathrm{~B} 1=\mathrm{Y} 10 \\
& \mathrm{~B} 2=\mathrm{Y} 20+\mathrm{u} 2
\end{aligned}
$$




\section{Mixed Model}

Log Cortisol TSST $-\mathrm{C}=\mathrm{Y} 00+\mathrm{Y} 10^{*}$ Linear Time + Y20* Quadratic Time $+\mathrm{u} 0+\mathrm{u} 2 *$ Quadratic Time $+\mathrm{r}$ 
Table 7. Fixed effect estimates from the final 2-level multilevel model with maternal-reported internalizing problems predicting log transformed cortisol levels in the Trier Social Stress Test.

\begin{tabular}{lccc}
\hline Fixed Effect & $\gamma$ & $S E$ & $t$ \\
\hline Intercept & .57 & .04 & $13.46^{* * *}$ \\
Linear time & .008 & .007 & 1.18 \\
Quadratic time & -.02 & .008 & $-2.27^{*}$ \\
\hline Random Effect & Variance & Standard & Chi-Square (df) \\
& & Deviation & \\
\hline Intercept & .06 & .25 & $502.64(39)^{* * *}$ \\
Quadratic Time slope & .001 & .04 & $111.93(39)^{* * *}$ \\
Level-1 & .03 & .17 & \\
\hline
\end{tabular}

${ }^{*} p<.05,{ }^{* *} p<.01, * * * p<.001$ 
Competition Challenge. Two separate models were used to examine the effect of child selfreported depressive symptoms and maternal-reported internalizing behaviours on child cortisol trajectories in the competition challenge.

Child-reported depressive symptoms predicting cortisol in the competition challenge. The final model fit the data significantly better than the null model $\left(\chi^{2}(3)=18.69, p<.001\right)$. The final model (shown in equation 3) accounted for $67 \%$ of the variance between individuals. At Level 1 , the fixed effect for time as a quadratic function significantly improved model fit. The random effect (slopes) for quadratic time was significant $\left(\chi^{2}(45)=61.53, p=.05\right)$ and significantly improved model fit, and thus slopes were allowed to vary across quadratic time in the model. The fixed effect of linear time was retained in the model, although it was not significant. The fixed effect of sAA as well as the interactions between sAA and linear and quadratic time were also entered at Level 1, and although they were not significant they were retained to test for potential higher level interactions (see Appendix 4.3). At level 2, CDI as well as the interaction between CDI and Level 1 predictors (i.e., CDI x sAA, CDI $\mathrm{x}$ linear time, CDI x quadratic time, CDI x linear time x sAA, CDI x quadratic time x sAA) were entered as fixed effects (see Appendix 4.3). The three-way interaction between CDI x linear time x sAA approached significance (efficient estimate $=.02, \mathrm{SE}=.01, p=.07$ ). Improvement in model fit approached significance, $\chi^{2}(1)=3.27, p=.07$, and $1.5 \%$ additional variance was accounted for (Appendix 4.3). Given that this interaction was not significant or did not significantly improve model fit, it was removed from the model. No other Level 2 main effects or interactions were significant or significantly improved model fit. All non-significant Level 1 and Level 2 effects were removed from the model (Appendix 4.3). The final model (Equation 3) included linear and quadratic time as fixed effects, and quadratic time as a random effect (Table 8). All of the tested covariates were nonsignificant, and thus were removed from the final model. 


\section{Equation 3:}

Level-1 Model

Log Cortisol COMP $=$ B0 + B1*Linear Time + B2*Quadratic Time $+r$

Level-2 Model

$\mathrm{B} 0=\mathrm{Y} 00+\mathrm{u} 0$

$\mathrm{B} 1=\mathrm{Y} 10$

$\mathrm{B} 2=\mathrm{Y} 20+\mathrm{u} 2$

Mixed Model

Log Cortisol COMP $=$ Y00 + Y10*Linear Time + Y20* Quadratic Time $+\mathrm{u} 0+$ $\mathrm{u} 2 *$ Quadratic Time $+\mathrm{r}$ 
Table 8. Fixed effect estimates from the final 2-level multilevel model with child-reported depression predicting log transformed cortisol levels in the Competition challenge.

\begin{tabular}{lccc}
\hline Fixed Effect & $\gamma$ & $S E$ & $t$ \\
\hline Intercept & .43 & .03 & $12.51^{* * *}$ \\
Linear time & -.0001 & .01 & -0.04 \\
Quadratic time & .01 & .004 & $3.27^{* *}$ \\
\hline Random Effect & Variance & Standard & Chi-Square (df) \\
& & Deviation & \\
\hline Intercept & .05 & .22 & $611.45(45)^{* * *}$ \\
Quadratic Time slope & .0002 & .02 & $61.54(45)^{*}$ \\
Level-1 & .02 & .15 & \\
\hline
\end{tabular}

Note. $\mathrm{CDI}=$ Child Depression Inventory; sAA = salivary alpha amylase.

$\mathfrak{t}=.075, * p<.05, * * p<.01, * * * p<.001$ 


\section{Maternal-reported internalizing behaviours predicting cortisol in the competition challenge.}

The final model fit the data significantly better than the null model $\left(\chi^{2}(1)=38.68, p<.001\right)$. The final model (shown in equation 4 ) accounted for $63 \%$ of the variance between individuals. Model coefficients and standard errors of fixed effects are shown in Table 8. At Level 1, the fixed effect for time as a quadratic function significantly improved model fit. The random effect (slopes) for quadratic time was significant $\left(\chi^{2}(41)=81.81, p<.001\right)$ and significantly improved model fit, and thus slopes were allowed to vary across quadratic time in the model. The fixed effect of linear time was retained in the model, although it was not significant. At Level 1, the fixed effects of sAA as well as the interactions between sAA and linear and quadratic time were not significant, however they were retained to test for potential higher level interactions (see Appendix 4.4).

At Level 2, maternal-reported internalizing behaviours (CBCL), as well as the interactions between CBCL and Level 1 predictors were entered as fixed effects. The CBCL x sAA interaction was significantly different from zero $(p<.05)$, indicating that coordination between cortisol and sAA varies across levels of internalizing behaviours. The addition of the CBCL x sAA interaction led to significantly improved model fit, $\chi^{2}(1)=5.05, p<.05$. All other Level 2 interactions were not significant (Appendix 4.1) and thus were removed from the final model. Covariates were also added at Level 2, but were not significant, and thus were removed from the final model (Table 9).

\section{Equation 4:}

Level-1 Model

Log Cortisol TSST-C = B0 + B1*Linear Time + B2*Quadratic Time + $\mathrm{B} 3{ }^{*} \mathrm{sAA}+\mathrm{r}$

Level-2 Model

$\mathrm{B} 0=\mathrm{Y} 00+\mathrm{Y} 01 *$ Maternal-reported internalizing $(\mathrm{CBCL})+\mathrm{u} 0$

$\mathrm{B} 1=\mathrm{Y} 10$

$\mathrm{B} 2=\mathrm{Y} 20+\mathrm{u} 2$

$\mathrm{B} 3=\mathrm{Y} 30+\mathrm{Y} 31 * \mathrm{CBCL}$ 


\section{Mixed Model}

Log Cortisol TSST $-\mathrm{C}=\mathrm{Y} 00+\mathrm{Y} 01 * \mathrm{CBCL}+\mathrm{Y} 10 *$ Linear Time + Y20*Quadratic Time + Y30*sAA + Y31*CBCL x sAA $+\mathrm{u} 0+$ $\mathrm{u} 2 *$ Quadratic Time $+\mathrm{r}$ 
Table 9. Fixed effect estimates from the final 2-level multilevel model with maternal-reported internalizing problems predicting log transformed cortisol levels in the Competition challenge.

\begin{tabular}{lccc}
\hline Fixed Effect & $\gamma$ & $S E$ & $t$ \\
\hline Intercept & .454 & .03 & $14.70^{* * *}$ \\
Internalizing & -.002 & .005 & -0.30 \\
Linear time & -.001 & .01 & -0.13 \\
Quadratic time & .01 & .007 & 1.84 \\
sAA & .01 & .10 & 0.08 \\
sAA* Internalizing & .05 & .02 & $2.24^{*}$ \\
& & & \\
\hline Random Effect & Variance & Standard & Chi-Square (df) \\
& & Deviation & \\
\hline Intercept & .03 & .18 & $351.81(40)^{* * *}$ \\
Quadratic Time slope & .001 & .03 & $81.81(41)^{* * *}$ \\
Level-1 & .02 & .14 & \\
\hline
\end{tabular}

Note. $\mathrm{sAA}=$ salivary alpha amylase.

${ }^{*} p<.05,{ }^{* *} p<.01, * * * p<.001$ 
In order to better understand the CBCL x sAA interaction, a multilevel model interaction computation tool was used (Preacher, Curran, \& Bauer, 2006). Conditional values of the mean, as well as 1 standard deviation (SD) above and below the mean were selected for the maternal-reported internalizing behaviour predictor. Simple slope analyses for the significant two-way interaction between sAA and internalizing behaviours predicting salivary cortisol, demonstrated a significant association between salivary cortisol and sAA (i.e., coordination) at high levels of internalizing behaviours $(\beta=0.63, \mathrm{SE}=.29, z=2.18, p<.05)$ and at moderate levels of internalizing behaviours $(\beta$ $=0.36, \mathrm{SE}=.18, z=1.99, p<.05)$, but not at low levels of internalizing behaviours $(\beta=0.10, \mathrm{SE}=$ $.11, z=0.91, p=.36$ ) (Figure 8). As shown in Figure 8, for individuals with high or moderate levels of internalizing behaviours, high levels of sAA are associated with high cortisol levels, whereas low levels of sAA are associated with low cortisol levels. In contrast, for individuals with lower levels of internalizing behaviours, the level of sAA (high or low) does not impact the level of cortisol (black line). 


\section{Internalizing by sAA interaction in the Competition Challenge}

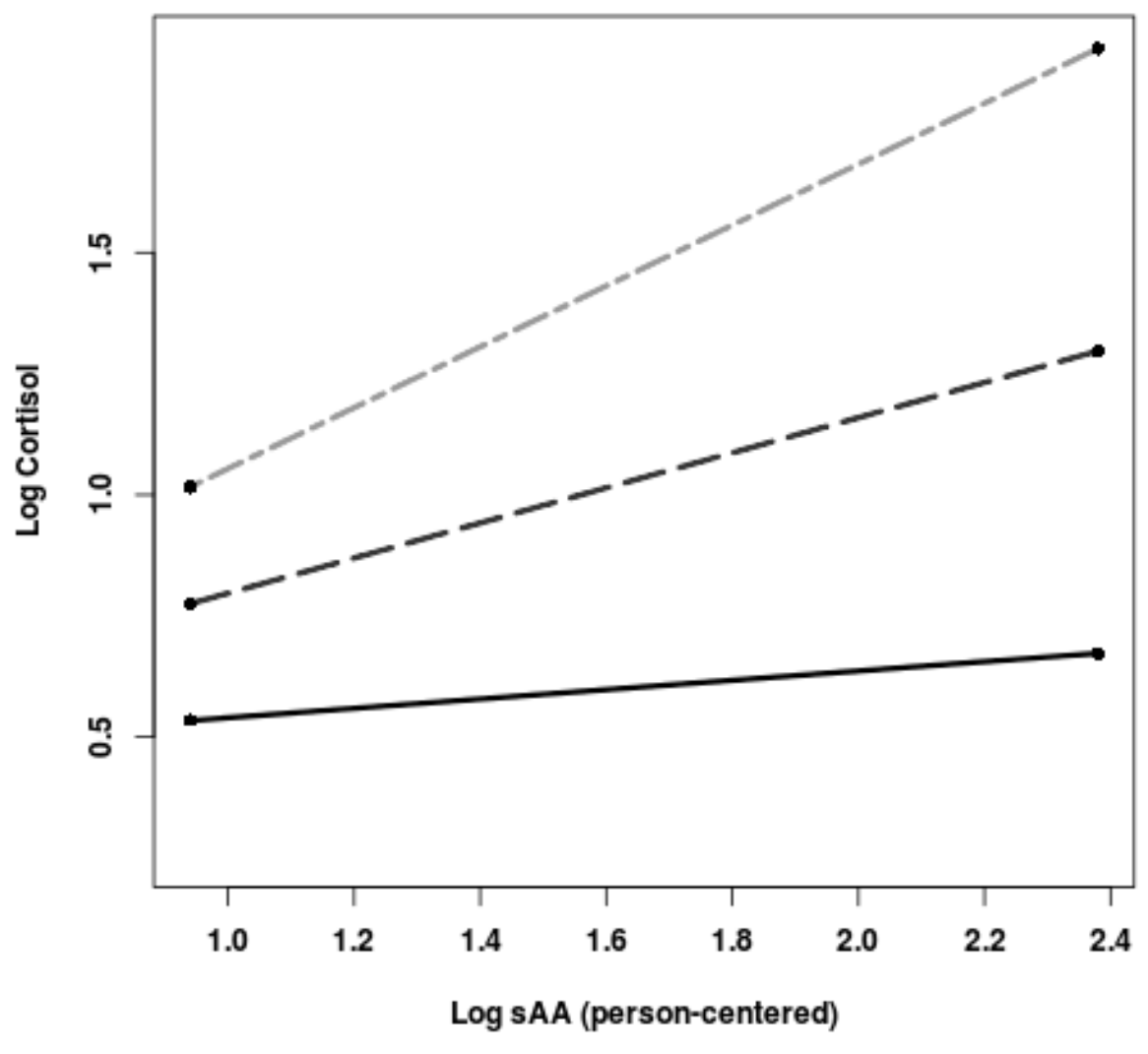

Figure 8. Interaction between maternal-reported internalizing problems and log transformed salivary alpha-amylase predicting log transformed cortisol levels in the competition challenge. 


\section{Discussion}

The present study assessed cortisol secretion patterns in the context of child internalizing problems while accounting for three primary factors that confound associations between cortisol and internalizing behaviours: 1) the differential effectiveness of stressor paradigms to elicit cortisol reactivity, 2) coordination between cortisol and sAA, and 3) the reporter (self or parent) of internalizing behaviour. This study assessed cortisol reactivity as it is related to internalizing behaviours as well as coordination with sAA in two stressor paradigms, which were specifically designed to differentially activate cortisol and sAA responses. The TSST-C is a frequently used, uncontrollable, social-evaluative stressor, which elicits strong cortisol responses in healthy children and adults (e.g., Dickerson \& Kemeny, 2004; Gunnar et al., 2009). In contrast, I developed a less-potent competition-based challenge, composed of a puzzle and mirror-tracing task. This competition challenge was selected in an attempt to elicit a strong SAA response, given that the SAM system (and sAA as a result) is theorized to be more strongly activated by controllable, effort-based challenges (Laurent, Powers \& Granger, 2013; Lundberg \& Frankenhaeuser, 1980). In addition, given prior inconsistencies in parent and child reports (e.g., Hartman et al., 2013), I examined internalizing behaviours using both the child-reported CDI and the maternal-reported Internalizing subscale of the CBCL. This is the first study to simultaneously address the three aforementioned confounds in the cortisol literature. Findings partially support the hypothesis that internalizing behaviours are associated with cortisol reactivity and cortisol—sAA coordination, however, findings differ based on the challenge and the reporter (mother or child) of internalizing behaviours.

\section{Summary of Findings}

In order to appreciate the findings as they specifically relate to internalizing behaviours, it is important to examine the general pattern of findings within the sample as a whole. Overall, mean 
cortisol and sAA levels across the entire sample suggest that the TSST-C is a potent stressor that elicits both cortisol and sAA reactivity (Figure $5 \mathrm{~A} \& \mathrm{~B}$, grey dashed line). In contrast, in the competition challenge, cortisol declines from baseline to 20 minutes and then returns to baseline levels at 60 minutes, whereas sAA declines steadily throughout the challenge (Figure 5 A \& B, black solid line). Taken together, it appears that cortisol and sAA increased in response to the TSST-C, whereas, cortisol and sAA declined in response to the competition challenge.

Consistent with our findings, many prior studies suggest that the TSST-C results in a strong cortisol and sAA response (e.g., Granger et al., 2006; Rohleder et al., 2004; Nater et al., 2006; as detailed below). In contrast, given that the competition challenge was designed to activate the SAM system, I did not expect that sAA would decline in this challenge. Prior research suggests that the SAM system is activated by controllable, self-paced, and effortful challenges (Lundberg \& Frankenhaeuser, 1980). In line with this initial research, Laurent et al. (2012) found that infants demonstrated a strong sAA response to the clean-up task and a less pronounced sAA response to the separation stressor. Although the competition challenge in the present study was designed to be effort-based and controllable, for the majority of the sample, it did not elicit a strong sAA response. Given that the competition challenge is a new procedure, it is possible that the level of effort (or lack there of) elicited by this particular challenge contributed to the declining sAA response.

Several other studies show mixed findings in terms of which types of challenges elicit sAA reactivity. For example, studies that employ uncontrollable and distressing challenges, such as a noise burst paired with an arm restraint (Shea et al., 2006), and viewing negative affect-inducing pictures (van Stegeren et al., 2008), result in increased sAA reactivity, however, other studies that employ similar procedures, such as noise (Morrison et al., 2003) or response to conflict vignettes (Koss et al., 2014) did not result in increased sAA reactivity. In addition, individual differences, such as age, might 
impact sAA reactivity across challenges. For example, Stroud et al. (2009) found that school-aged children did not mount strong sAA reactivity in response to either a performance-based or interpersonal challenge. In contrast, adolescents mounted a strong sAA response to the interpersonal challenge but not the performance challenge. This suggests that age might impact which types of challenges activate sAA responsivity. Perhaps the nature of the competition challenge in the present study was not potent enough to elicit a sAA response in our sample of school-age children. Given that this is the first study to use the mirror tracing and puzzle tasks to compose the competition challenge, it remains unclear if the declining sAA response is related to the nature of the stressor or sample characteristics (e.g., age). Overall, it remains unclear which types of challenges elicit a strong vs. weak sAA response, given the limited number of studies that assess sAA across multiple challenges. Additional research is required to understand the types of challenges that elicit relative differences in cortisol and sAA responsivity.

Interestingly, although the competition challenge is not effective in eliciting a strong sAA response across the entire sample, there is a significant correlation between sAA and cortisol $(r=.20)$ in the competition challenge, suggesting weak but significant coordination in this challenge. In comparison, there was not a significant correlation between cortisol and SAA $(r=-.10)$ in the TSST-C. The significant correlation between cortisol and sAA in the competition challenge but not the TSST-C may reflect the similar trajectories of these two analytes in the competition challenge. That is, both cortisol and sAA decline across the first four sample points in the competition challenge, whereas cortisol and sAA show different patterns of change in the TSST-C. Further this is in line with the proposed hierarchical activation of the HPA and SAM systems, that is, very potent and distressing challenges (e.g., TSST-C) elicit an initial SAM response followed by a strong HPA response, whereas less potent challenges may not require strong HPA or SAM activation, and may be sufficiently managed by the parasympathetic nervous system (Del Giudice et al., 2011). Although these 
correlations tell us something about the overall associations between cortisol and sAA, more refined analyses are required to adequately understand the nature of physiological coordination.

To investigate whether internalizing behaviours predicted cortisol reactivity and cortisol-sAA coordination, MLM analyses were conducted. In total, this study utilized four separate MLM analyses to examine cortisol reactivity in two challenges (TSST-C and competition) and using two types of internalizing behaviours (maternal-reported CBCL internalizing behaviour and child-reported CDI difficulties).

Trier Social Stress Test- Child Version. In the TSST-C stressor, I found a significant CDI $\mathrm{x}$ time interaction, such that children with higher self-reported depressive symptoms, compared to those with lower self-reported depressive symptoms, evinced a decline in cortisol over time throughout the TSST-C (Figure 6 and 7). This finding will be discussed in relation to prior research that examines associations between blunted cortisol reactivity and depression, as well as theoretical models of hypocortisolism. Unlike child-reported depressive symptoms, maternal-reported internalizing behaviours did not predict cortisol reactivity in the TSST-C. In addition, I did not find significant coordination between sAA and cortisol in the TSST-C.

Competition challenge. In the competition challenge, child-reported depressive symptoms did not significantly predict cortisol reactivity. However, maternal-reported internalizing behaviours significantly predicted coordination between SAA and cortisol, such that higher sAA predicted higher cortisol levels, but only for children with high or moderate levels of internalizing behaviours (Figure 8). These findings will be discussed in more detail as they relate to prior empirical studies as well as theoretical models of HPA and SAM coordination.

Associations between Child Depressive Symptoms and Cortisol Reactivity in the TSST-C 
Results indicate that cortisol trajectories declined across time for those with higher self-reported depressive symptoms, compared to those with lower depressive symptoms. More specifically, as shown in Figure 7, individuals with higher self-reported depressive symptoms showed an initial decline in cortisol, followed by a return to baseline levels, whereas, individuals with lower depressive symptoms showed an initial increase in cortisol, followed by return to baseline. This finding is consistent with prior research that demonstrates that adolescents who had higher depressive symptoms showed blunted cortisol reactivity in the TSST-C (Booij et al., 2013; Harkness et al., 2011). More specifically, Harkness et al. (2011) examined the combined role of clinical levels of depression and maltreatment history in predicting cortisol reactivity in a sample of adolescents. Adolescents with more severe depression exhibited a blunted cortisol response during the TSST-C, regardless of their maltreatment history; whereas, adolescents with mild/moderate depressive symptoms and a maltreatment history produced more prolonged cortisol reactivity. In addition, Booij et al. (2013), using a community sample of adolescents, examined how the chronicity of depressive symptoms (measured using self- and parentreported questionnaires) was associated with cortisol reactivity in the TSST. The findings suggested a curvilinear association between depression and cortisol reactivity, such that recent-onset depressive problems predicted an increased cortisol response, and more chronic depressive problems predicted a blunted cortisol response. Further, the present findings in combination with these aforementioned studies, suggest that depressive symptoms are associated with blunted cortisol reactivity in both community and clinical samples. As outlined above, most prior research that examines associations between internalizing behaviours and cortisol reactivity in the TSST-C, is conducted with adolescent samples. Therefore, less is known about how internalizing behaviours predict cortisol reactivity in the TSST-C in school-age children. 
The present findings extend prior research by demonstrating that higher depressive symptoms are associated with blunted cortisol reactivity in the TSST-C, in a sample of 8-10 year-old children. To my knowledge, this is the first study to demonstrate the specific association between depressive symptoms (not broader internalizing behaviours) and cortisol reactivity to the TSST-C in a sample of 8-10 year olds. Given that research shows a marked increase in depression after the onset of puberty and throughout adolescence (Patton et al., 2008; Stroud et al., 2011), the current findings suggest that blunted cortisol reactivity may also be associated with depressive symptoms earlier in childhood (prior to puberty), which potentially has important implications for future basic and applied research (discussed in detail below). Future longitudinal research is needed to determine whether associations between internalizing behaviour and cortisol reactivity in middle childhood is predictive of subsequent difficulties.

The current findings that children with higher self-reported depressive symptoms show blunted cortisol reactivity in the TSST-C can be understood in the context of allostatic load. Allostasis is the process by which diverse physiological and neurobiological systems adjust and adapt in response to environmental changes, to achieve homeostasis (McEwen, 1998; McEwen \& Seeman, 1999). However, over time, repeated stress may result in the "wear and tear" of the stress response system (allostatic load). As outlined by McEwan (1998), both prolonged stress responses (increased cortisol secretion and failure to recover) or blunted/inadequate responses are considered to be maladaptive markers of allostatic overload. McEwan also outlines that blunted HPA activity may result in altered activity of adjoining stress systems (e.g., SAM system, immune system), which can lead to adverse health outcomes. It is important to note that although allostatic load has often been conceptualized within the context of repeated life stress (and often trauma), I did not measure these variables directly in the present study. In fact, the current sample is relatively low-risk. Here, I am using the allostatic 
load framework simply to interpret the blunting cortisol reactivity findings as they relate to internalizing behaviours, and to understand how these associations are potentially related to life stress.

Prior research suggests that hypocortisolism (i.e., blunted cortisol response) is associated with a number of physical illnesses and mental health problems in adults, including depression and PostTraumatic Stress Disorder (Fries, Hesse, Hellhammer, \& Hellmammer, 2005; Heim, Ehlert \& Hellhammer, 2000). It is hypothesized that hypocortisolism occurs in response to repeated exposure to stress (discussed in more detail below). It has been proposed that blunted cortisol could result from previous periods of high cortisol earlier in development, including in the prenatal period and early childhood (Charmandari, Kino, Souvatzoglou, \& Chrousos, 2003). For example, pre-pubescent children demonstrated blunted cortisol responses in face of stressful family circumstances (Hankin, Badanes, Abela, \& Watamura, 2010). It was concluded that this attenuation prevented children from having to repeatedly mount a cortisol reaction in response to repeated and uncontrollable daily stressors.

In addition to being associated with hypocortisolism, exposure to stressful life events, both major and minor, is one of the most strongly associated risk factors for depression across the lifespan. With regard to developmental psychopathology, extensive research has highlighted the importance of psychosocial stress in the development and maintenance of internalizing problems (e.g., Cicchetti \& Toth, 1991; Haggerty, Sherrod, Garmezy, \& Rutter, 1994). For example, extreme stressful life events, such as the death of a parent or severe maltreatment, are associated with higher levels of depression in youth (Kendler, Kuhn, \& Prescott, 2004; Williamson, Birmaher, Dahl, \& Ryan, 2005). In addition, less severe stressful life events, such as daily hassles (O'Sullivan, 2004), are also associated with increased depressive problems. It is important to note that stressful life events do not induce depressive symptoms in all individuals (e.g., Bonanno, 2004). Rather, diathesis stress models of depression 
(Hammen, 2005) posit that preexisting vulnerability factors potentiate risk for depression during stressful life events. Extensive research has examined atypical HPA activity as a key biological risk factor that potentiates risk in the face of stressful life events (Colich, Kircanski, Foland-Ross, \& Gotlib, 2015; Foland-Ross et al., 2013). Taken together, independent research suggests that stressful life events, major and minor, are associated with hypocortisolism as well as the etiology and maintenance of internalizing behaviours. Again, although allostatic load is proposed as a potential mechanism that may account for the associations between life stress, depression, and blunted cortisol activity, this study did not directly assess life stress or trauma, and thus it is unclear whether allostatic load actually accounts for the present findings.

Although research shows that hypoactive HPA activity as a result of allostatic overload is maladaptive, additional theory and research outlines how repeated stress may result in blunted cortisol responsivity. Heim and colleagues (2000) propose a model that consists of several potential biological mechanisms and psychological and environmental determinants of hypocortisolism. In particular, potential mechanisms that underlie the development and maintenance of hypocortisolism include: 1) reduction in the synthesis of different agents of the HPA axis (i.e., cortiscortropin releasing factor (CRF), ACTH, cortisol); 2) initial hypersecretion which results in adaptive down regulation of CRF receptors; 3) increased feedback sensitivity of the HPA axis, due to initial increased levels of circulating cortisol; and 4) morphological changes to the adrenal gland (Heim et al., 2000).

In addition to these biological mechanisms, Heim et al. noted several determinants that impact hypocortisolism. Of particular importance to the present findings, the characteristics of the stressor (including the nature, frequency, intensity, controllability and predictability) and the individual's coping style likely impact hypocortisolism. A detailed discussion of these determinants, as well as the supporting research, is beyond the scope of this discussion (refer to Heim et al., 2000). However, 
through the design of this study, I have directly addressed how the characteristics of the stressor impact cortisol reactivity. In particular, the TSST-C is designed to be an intense social-evaluative stressor that is deemed to be uncontrollable and unpredictable, and evokes a strong HPA response (e.g., Dickerson \& Kemeny, 2004). This compares to the competition challenge, which is designed to be more effortbased and controllable.

In addition, I will briefly elaborate on how one's coping style can impact hypocortisolism, as this determinant is particularly relevant to the association between blunted cortisol and depression. Environmental stress at some point in time and to some degree is inevitable. A person's coping style may mediate the development of a maladaptive stress response, in particular, hypocortisolism. Research suggests that passive coping, denial, and withdrawal are specifically associated with hypocortisolism (Katz, Weiner, Gallagher, \& Hellman, 1970; Wolff, Hofer, \& Mason, 1964). Interestingly, the same symptoms that are associated with hypocortisolism also characterize depression. For example, trait rejection sensitivity was associated with both depressed mood and hypocortisolism in young women (Tops, Riese, Oldehinkel, Rijsdijk, \& Ormel, 2008). Therefore, our finding that children with higher depressive symptoms experience blunted cortisol reactivity can be understood in the context of hypocortisolism, a marker of allostatic overload, which can result from an interchange between stressful life events and ineffective coping.

Flexibility of cortisol responsivity. This study was designed to assess whether internalizing behaviours have distinct associations with cortisol reactivity in two differentially potent challenges. Although I was unable to statistically compare results across studies, given the nature of the study design and for the sake of completeness, these findings will be discussed as they relate to flexibility. Prior research from our group (e.g., Atkinson et al., 2016) has highlighted the importance of assessing cortisol flexibility, given that distinct stressors differentially elicit cortisol responses, and individual 
psychological variables predict adaptive cortisol responsivity (i.e., high response to potent challenge, low response to non-potent challenge). Ideally, the present study would have directly examined cortisol flexibility by statistically comparing cortisol trajectories across the two challenges. However, this would have resulted in 5 main effects and 11 interactions in the MLM model, which was not feasible with our sample size. Unfortunately, there is not a rule of thumb for the number of individuals needed to have sufficient power to detect effects in MLM models. The sample size at the highest level (i.e., Level 2, individuals in the present study) is the main limiting factor of the design (Snijders, 2005). In addition, larger clusters of Level 2 variables are required when testing random effects (slopes and intercepts) in addition to fixed effects (Snijders, 2005). To gain a rough estimate, I look to power recommendations in multiple regression analyses. In multiple regressions, a minimum of 20 subjects per variable is recommended (i.e., over 300 subjects needed to test the current model). However, given that interaction terms only account for residual variance and that more power is required when testing random effects, this is likely a very low estimate. Although the small study sample prevented me from statistically assessing whether internalizing behaviours differentially predicted cortisol reactivity across these two challenges (i.e., flexibility), I experimentally controlled for the potency of the challenge, thereby allowing for more methodological clarity when interpreting my findings.

Findings indicate that child-reported depressive symptoms were associated with blunted cortisol reactivity in the TSST-C, however I did not find significant associations between child-reported depressive symptoms and cortisol levels in the competition challenge. It is possible that I did not find a significant association between depressive symptoms and cortisol in the competition challenge because of limited power to detect effects. Alternatively, it is possible that the nature of this challenge simply did not elicit a differential cortisol response between those with high or low depressive symptoms. In relation to the above discussion of hypocortisolism and allostatic load, it is conceivable that children 
with higher self-reported depressive symptoms demonstrate blunted cortisol responses across both challenges, and I did not find a statistical difference in cortisol trajectories in the competition challenge because children with low levels of depression also demonstrated a decline in cortisol in the competition challenge (see Figure 9). In line with this interpretation, based on the visual inspection of these graphs, it appears that children with higher levels of self-reported depression evince declining cortisol responses in both challenges. These are intriguing findings that are consistent with prior work and potentially support the notion of cortisol flexibility. In our study, an adaptive cortisol response, would be indicated by a cortisol increase (followed by a return to baseline) in the potent TSST-C and a steady decline in cortisol in the non-potent competition challenge, which is shown in the overall sample trajectories (Figure 5). 


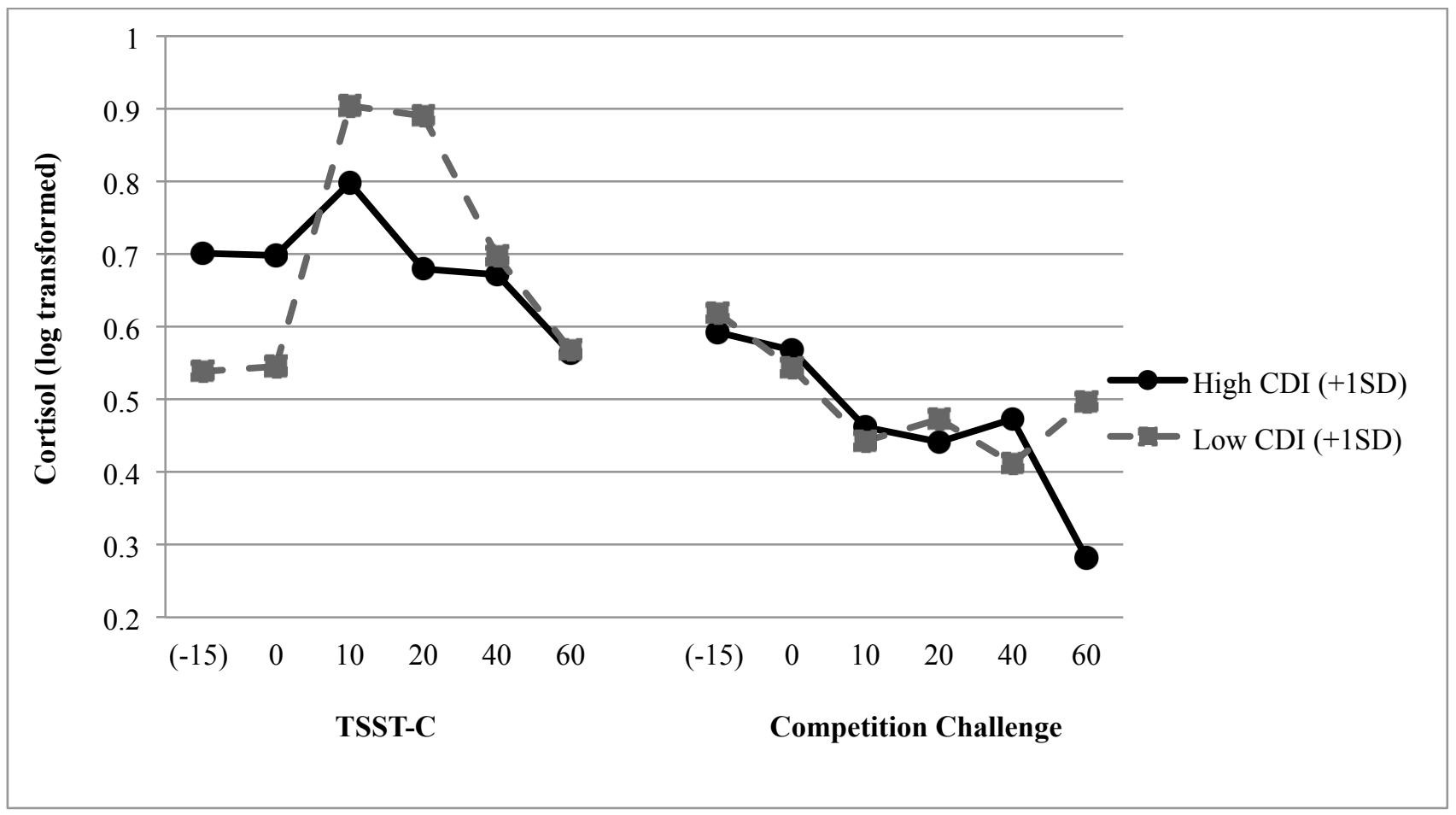

Figure 9. Visual depiction of cortisol trajectories in the Trier Social Stress Test-Child Version (TSSTC) and Competition challenge, for those with high (+1 SD) and low (-1 SD) self-reported depressive symptoms (measured using the Child Depression Inventory; CDI). 
Only two prior studies (Laurent et al., 2015, 2016) have examined the association between internalizing problems and cortisol reactivity across two distinct challenges. Laurent et al. (2015) demonstrated that specific child anxiety problems had unique associations with cortisol reactivity in the TSST-C and a problem solving challenge. More specifically, generalized anxiety was associated with elevated cortisol in both challenges, specific phobia was associated with elevated cortisol levels in the TSST-C, and OCD was associated with limited cortisol recovery after the problem-solving challenge. In addition, Laurent et al. (2016) examined broader maternal-reported internalizing problems as they related to cortisol reactivity in a sample of 8-16 year olds who participated in a performance (similar to the TSST-C) and an interpersonal rejection challenge. Interestingly, Laurent et al. found that overall maternal-reported internalizing symptoms predicted an earlier cortisol peak in the interpersonal stressor, however in contrast to the present study findings, internalizing behaviours were not associated with cortisol reactivity in the performance challenge. Although the total scale score of internalizing symptoms was not predictive of cortisol reactivity in the performance challenge, anxiety-specific symptoms were related to high cortisol levels during this challenge. These findings were also moderated by sex, such that for females only, anxiety was associated with a later cortisol peak during the performance challenge. Taken together, the findings of the current study, along with these two prior studies, suggest that the associations between internalizing behaviours and cortisol reactivity differ depending on the nature of the stressor paradigm, and perhaps the type of internalizing behaviour (e.g., depression or anxiety). Nonetheless, future work with a larger sample is needed to directly assess whether internalizing problems differentially predict cortisol reactivity across two distinct challenges.

\section{Coordination of Cortisol and sAA}

In addition to examining cortisol reactivity, the current study aimed to assess coordination between cortisol and sAA, within two distinct challenges. It is well established that the HPA and SAM 
systems operate in parallel to produce a coordinated physiological response to stress; however, as stated by El-Sheikh et al. (2008), "the optimal type of co-action between these systems is not wellunderstood" (p. 603). The current findings indicate that children with higher maternal-reported internalizing behaviours who experienced higher sAA also experienced higher cortisol in the competition challenge. It is important to note that unlike our cortisol reactivity findings (i.e., depressive symptoms predicted a blunted cortisol trajectory in the TSST-C), these coordination findings are based on mean levels of cortisol and sAA, not trajectories. Given the three-way interaction between time, sAA and internalizing behaviours was not a significant predictor of cortisol levels, I cannot say that coordination between sAA-cortisol trajectories varied by internalizing behaviours, I can only discuss coordination between overall (average) cortisol-sAA levels. In addition, in contrast to the significant physiological coordination in the competition challenge, I did not find significant cortisol—sAA coordination in the TSST-C. It is unclear why the competition challenge and not the TSST-C elicited strong coordination. However, I attempt to explain these findings in the context of prior theory and research.

These results can be understood in the context of the additive model of HPA and SAM activation proposed by Bauer et al. (2002). The additive model suggests that the HPA and SAM systems operate in conjunction, with optimal functioning occurring when each system is activated at a moderate level or one system is highly activated while the other is not (i.e., asynchronous activity), and thus synchronously high (or low) activation in both systems is maladaptive (Bauer et al., 2002). In the present case, in the competition challenge, high activation in both systems is associated with higher levels of internalizing behaviour problems. Although somewhat counter-intuitive given the literature associating internalizing behaviour problems with blunted cortisol reactivity (including our current findings that higher self-reported depressive symptoms are associated with blunted cortisol reactivity in 
the TSST-C), the nature of the competition challenge may explain why internalizing behaviour problems are associated with higher coordination (high cortisol and high sAA) in this challenge (discussed below).

In addition, these findings can be understood within the context of the ACM, and partially support the vigilant phenotype from the ACM (Del Giudice et al., 2011). The ACM proposes that individual differences in stress responsivity result from adaptation to environmental conditions. The vigilant phenotype is characterized by high reactivity of both the HPA and SAM systems, and evolves from a life history of being exposed to highly stressful environments that consist of repeated external threats. Therefore, individuals with the vigilant pattern of responsivity adapt to their stressful environments by mounting high cortisol and sAA activity, indiscriminately, across contexts (whether these contexts are in fact, threatening and dangerous). Our present findings only partially support the vigilant stress-response profile, because internalizing behaviours were only associated with high cortisol and high sAA reactivity in the competition challenge, not the TSST-C. Therefore, rather than internalizing behaviours being associated with an indiscriminant hyper-active response across contexts, it appears that coordination between systems might be context-specific.

This coordination finding is particularly interesting given that, for the overall sample, both cortisol and sAA tended to decline in the competition challenge (see Figure 5, black solid lines). The competition challenge used in the current study was specifically designed to be a non-potent, controllable and effort-based challenge. Although I expected this challenge to elicit a sAA response (given that it is effort-based), this challenge was not effective in evoking an increase in cortisol or sAA, in the overall sample. This suggests that although the majority of the sample experienced a decline in cortisol and sAA in this non-potent challenge, those with higher internalizing behaviour problems experienced synchronous higher activation of both the HPA and SAM systems. In order to understand 
how these findings relate to previous studies on coordination, it is important to consider the type of challenge used to elicit stress responses. In the context of our competition challenge, a typical response appears to be one of low cortisol and sAA responsivity (Figure 5), however, in more potent challenges (as has been used in previous studies, discussed below) a typical/adaptive response might be higher cortisol and sAA responsivity.

It is difficult to compare the present study results to other coordination findings in the literature, because other studies use different types of challenges, and no previous study has examined coordination across multiple challenges. Similar to my results, El-Sheikh et al. (2008) found that that synchronously high levels of cortisol and sAA coordination were associated with higher internalizing problems. However, El-Sheikh et al. used a composition of challenges to elicit stress, including a cognitive assessment, listening to a recording of two adults arguing, and a star-tracing activity. Given a composition of challenges were used (some of which overlap with the TSST-C and competition challenge used in the present study), it is unclear what aspect of the challenge effectively elicited coordination.

Interestingly, Bae et al. (2015) used the potent TSST-C challenge and found that higher levels of internalizing behaviours were associated with lower cortisol and lower sAA. Although I did not find significant coordination in the TSST-C, these findings may be related to the present study results for the competition challenge. At the surface, the current findings that internalizing behaviours were associated with higher cortisol and higher sAA in the competition challenge seem contradictory to Bae et al.'s findings, because they found that internalizing behaviours were associated with lower cortisol and lower sAA. Although I recognize that, unlike Bae et al., I did not find coordination in the TSST-C, the present study findings for the competition challenge may be in line with this prior research. Our less-potent challenge elicited coordination at the high level (i.e., high cortisol and high sAA) for 
children with higher internalizing behaviour problems, which is interpreted as an atypical response in comparison to overall sample trajectory (see Figure 5). Bae et al. used the potent TSST-C challenge to elicit coordination at the low level (i.e., low cortisol and low sAA for children with higher internalizing behaviour problems), a pattern of coordination that was similarly interpreted as a non-adaptive/atypical response. Therefore, when taking into account the difference between the TSST-C and the competition challenges, our findings may be partially consistent with those of Bae and colleagues. Of course, our non-significant coordination findings in the TSST-C directly contradict Bae et al.'s work. The present inconsistent findings highlight the importance of assessing how internalizing behaviour problems impact coordination across variant stressors.

The current results only indicate significant cortisol—sAA coordination in the competition challenge, and not in the TSST-C. It is unclear why the competition challenge and not the TSST-C would elicit strong coordination. In particular, Del Giudice et al. (2011) proposed that potent stressors result in the activation of both stress systems, so one might predict that the potent TSST-C would elicit high coordination. In line with this theory, Bae et al. demonstrated significant cortisol—sAA coordination in the TSST-C. It is important to note, Bae et al. had a much larger sample $(\mathrm{N}=169)$ and thus significantly more power to detect a coordination effect in the TSST-C. Therefore, it is possible that this study's low sample size (and resulting low power), contributed to my inability to detect differences in coordination between those with varying levels of internalizing behaviours in the TSSTC. However, it is premature to speculate about the nature of cortisol and sAA coordination as it relates to internalizing behaviours, particularly as it is assessed in different stressor contexts, because this literature is in its infancy and is ambiguous to date.

\section{The Reporter of Internalizing Behaviours Impacts Associations with Cortisol Responsivity}


In addition to controlling for the stressor paradigm and cortisol-sAA coordination as confounds, this study was also designed to control for different raters of internalizing behaviours. The results indicate that child-reported depressive symptoms predicted lower cortisol reactivity in the TSST-C, whereas maternal-reported internalizing behaviours predicted coordination between cortisol and sAA in the competition challenge. Within the overarching developmental psychopathology literature, it is well known that parent and child reports of internalizing behaviours often do not align (Achenbach \& Rescorla, 2001; De Los Reyes \& Kazdin, 2005). In the current study, child-reported depressive symptoms were not significantly correlated with maternal-reported internalizing behaviours $(r=.18, p$ $=.24)$. Given the low correlation, it understandable that parent and child reports act as unique predictors of cortisol reactivity. This inconsistency in parent-reported and child-reported associations is in line with previous research. For example, using the TSST-C, Hartman et al. (2013) found that child-reported internalizing behaviours were significantly associated with cortisol, whereas maternalreported internalizing behaviours were not. In contrast, other research using a conflict discussion task, found that maternal-reported internalizing behaviours interacted with family stress to predict cortisol reactivity (Steeger et al., 2017). This suggests that a combination of the informant of internalizing behaviours and the type of stressor used to evoke a cortisol response might be associated with these inconsistent findings. That is, child-reported difficulties may be more strongly associated with cortisol responsivity in certain types of stressors, whereas maternal-reported problems may be associated with stress reactivity in other kinds of stressors. Given that this is the first study to concurrently examine multiple informants of internalizing behaviours as they relate to cortisol reactivity in two distinct challenges (see Table 1), it is difficult to speculate on reasons and future work is needed to further investigate these associations. 
In addition, inconsistent findings may also be associated with the type of measure used, rather than the rater, per se. That is, in this study children completed a depression inventory, whereas mother completed a broader internalizing inventory. The CDI consists of items, which assess negative mood, low self-esteem, ineffectiveness and interpersonal problems, whereas the CBCL internalizing subscale includes items related to depressed mood, withdrawal, anxiety, and somatic symptoms. Various aspects of internalizing symptomatology, such as anxiety and somatic symptoms (e.g., Janssens et al., 2012; Laurent et al., 2015), are uniquely associated with cortisol responsivity. Thus, it is possible the different constructs captured with the CBCL and CDI account, at least it part, for the differing results. Taken together, these inconsistencies underscore the need to use multiple reporters of analogous internalizing problems (i.e., child and parent reports of depressive symptoms (CDI), or youth and parent reports of broader internalizing behaviour (YSR and CBCL)) when assessing the association between internalizing symptoms and child cortisol responsivity.

\section{Additional Considerations: Age and Sex}

The present findings should be discussed in relation to two important sample characteristics: age and sex. As outlined above, this study extends prior research by demonstrating that higher selfreported depressive symptoms are associated with blunted cortisol reactivity in the TSST-C and higher maternal-reported internalizing problems are associated with increased physiological coordination (higher sAA and higher cortisol) in a competition challenge, in a sample of 8-10 year-old children. This age group is unique in that it is between early childhood and the onset of puberty and adolescence. Although extensive research suggests that internalizing problems increase after the onset of puberty (e.g., Stroud et al., 2011), the present findings suggest that the risk for internalizing problems, at least at a physiological level, might begin prior to puberty. Ultimately, longitudinal research is required to 
determine if atypical cortisol reactivity in middle childhood is predictive of the chronicity and severity of internalizing problems in later childhood and adolescence.

Del Giudice, Angeleri, and Manera (2009) apply an evolutionary-based developmental psychobiological theory to suggest that the developmental transition from early to middle childhood (labeled as the juvenile transition) is a critical period of behavioural, psychological, and biological development. Although a comprehensive exploration of this theory is beyond the scope of this discussion, it is important to outline the unique physiological changes that occur during middle childhood, which underscore the need to study HPA and SAM activity in this age group. The transition to middle childhood often marks the onset of adrenarche, defined as a maturational phase that is characterized by hormonal and structural changes, which typically occurs at 7 years in western countries (although the age ranges between 5 and 10, depending on the country; Del Giudice et al., 2009). During this time period, the adrenal glands begin to secrete increased androgens (Dorn \& Rotenstein, 2004; Rainey, Carr, Sasano, Suzuki, \& Mason, 2002). In addition, the adrenal cortex increases in size and weight (Auchus \& Rainey, 2004; Dhom, 1973), with particular increases in size of areas of the adrenal cortex that produce dehydroepiandrosterone (DHEA), a sex hormone associated with HPA activity.

For example, cortisol affects the activity of the sex-hormones system and sex hormones (e.g., DHEA) regulate the activity of stress pathways (e.g., Tilbrook, Turner, \& Clarke, 2002). In addition, androgens and estrogens affect the stress system by directly influencing cortisol synthesis, as well as CRH synthesis in the hypothalamus (Viau, 2002; Viau, Bingham, Davis, Lee, \& Wong, 2005).

Related, this developmental period marks a transition in social buffering of cortisol responses. In contrast to infancy and early childhood, where parental figures act as the primary social buffer of a child's stress response (reviewed by Hostinar \& Gunnar, 2013), beginning in middle-childhood, 
children are able to utilize friends and parents equally as buffers for HPA activity (e.g., Doom, Doyle, \& Gunnar, 2016). For example, 7- to 12-year-old girls who had a phone conversation with their mothers a after the TSST-C demonstrated lower salivary cortisol levels post-stressor (Seltzer, Ziegler, \& Pollak, 2010). In addition, in a study with 10- to 11-year-olds, daily stressful events were associated with lower cortisol levels if children had a friend at the time of the stressful event (Adams, Santo, \& Bukowski, 2011). This suggests the emergence of a unique association between stress regulation and social relationships in middle childhood.

In addition, middle childhood marks a drastic change in children's cognitive and behavioural development. For example, between 5 and 7 years old, there is a marked increase in attention control, planning, reflection, and self-regulation abilities (Collins, 1984; Sameroff \& Haith, 1996; Weisner, 1996). Therefore, given the unique biological, social, and cognitive changes associated with middle childhood, this is an important sample to study, particularly when examining associations between stress physiology and the onset of mental health difficulties. Although most research focuses on infancy and adolescence, there has been a recent push to begun to assess cortisol reactivity within middle childhood (de Veld, Riksen-Walraven, de Weerth, 2012; 2014; Jaffe et al., 2015). Future research is needed to examine associations between stress physiology (including both HPA and SAM activity) and internalizing symptoms in middle childhood.

In addition, sex is an important sample characteristic associated with stress reactivity. In the present study, although females had higher mean cortisol levels in the TSST-C compared to males, there were no significant sex differences in cortisol in the competition challenge, and sex was not a significant covariate in any of the MLM models. In addition, neither self-reported depressive symptoms nor maternal-reported internalizing problems differed by sex. These null-findings related to sex might be associated with the age of the present sample. Some research suggests that prior to puberty there are 
few differences between males and females in HPA activity, and that sex differences become apparent during adolescence (Booth, Granger, \& Shirtcliff, 2008) and persist through adulthood (Kudielka \& Kirschbaum, 2005). In contrast, a recent meta-analysis suggests that sex differences are present earlier in childhood, with cortisol levels higher in boys than girls before the age of 8 and lower in boys compared to girls after the age of 8 (der Voorn et al., 2017). In line with this meta-analysis, individual research studies suggest that sex differences become present as early as middle childhood (Leppert, Kushner, Smith, Lemay \& Dougherty, 2016; Lopez-Duran, Hajal, Olson, Felt, \& Vazquez, 2009). Similarly, Laurent et al. (2016) found specific sex differences in cortisol reactivity as they relate to internalizing and externalizing behaviour problems in a sample of individuals aged 8 to 16 years old. It is possible that sex-specific differences in cortisol reactivity are associated with age, however it was also suggested by Laurent and colleagues (2016) that sex-specific stress differences are associated with the nature of the challenge, such that females respond more strongly to interpersonal stressors compared to performance stressors. This is a relatively novel area of study that requires much further investigation across age groups and distinct challenges.

\section{Study Strengths}

To date, research examining the association between cortisol reactivity and childhood internalizing behaviours is equivocal. This literature is complicated by several methodological downfalls and inconsistencies, including single stressor designs, absence of the examination of physiological coordination, and different raters of internalizing behaviours. The current study aimed to address these issues. To begin, this study assessed cortisol reactivity within two distinct stressors. The cortisol literature is plagued by inconsistent findings, which in many cases can be understood in relation to differentially stressful paradigms used across studies. This is only the third study to examine how internalizing behaviours are associated with cortisol responses in two different stressor paradigms, 
and this is the first to specifically examine these associations in a middle-childhood sample. Although I was unable to directly examine cortisol flexibility, because our low sample size limited statistical comparisons of cortisol reactivity across stressors, the methodological design of this study enables the exploration of how internalizing behaviours relate to cortisol reactivity in each of these two distinct challenges.

Second, given that the HPA axis does not operate in isolation, this study examined cortisol and sAA coordination. Research shows that the combination of cortisol to sAA is a better indicator of stress dysregulation compared to either index alone (Ali \& Pruessner, 2012). Although stress research is moving towards concurrent cortisol and sAA measurement, this is the first study to examine HPASAM coordination in two different stressors. This furthers our understanding of how the characteristics of the stressor may influence physiological coordination.

Third, given the low concordance between parent and child reports of psychopathology as well as the inconsistent findings between parent and child reported internalizing behaviour problems and cortisol reactivity, this study used both mother and child reports of internalizing behaviours. The use of multiple raters proved fruitful, as findings differed based on the reporter of symptoms.

In addition to examining these three main confounds, this study advances the literature by using a refined statistical approach to assess cortisol trajectories. Instead of using point estimates such as $\mathrm{AUC}_{\mathrm{G}}$ and $\mathrm{AUC}_{\mathrm{I}}$, this study used MLM to measure cortisol sampled across multiple time points. The use of cortisol trajectories enables the examination of the potential nuances in cortisol patterns (e.g., declining slopes). In addition, using MLM accounts for and examines intra-individual differences in intercepts and slopes of cortisol.

\section{Limitations and Future Directions}


This study is not without limitations that should be addressed in future work. First and foremost, this was a small sample to test complex multilevel models, which increases the likelihood of Type 1 and Type 2 errors. It is possible that our inconsistent findings (e.g., coordination in one stressor but not the other) were associated with limited power. Further, reduced power might explain why I only found mean level coordination (i.e., sAA $\mathrm{x}$ internalizing behaviour) and were not able to find coordination across cortisol and sAA trajectories (i.e., time $\mathrm{x}$ sAA $\mathrm{x}$ internalizing behaviour). It is a limitation of the study that the coordination findings are restricted to overall (mean level) associations between sAA and cortisol. Future work with a larger sample may be able to detect more refined coordination findings that relate to sAA and cortisol trajectories across time.

Related, given the small sample, I was not able to make statistical comparisons to examine cortisol flexibility. That is, I could not statistically assess how internalizing behaviour problems predicted intra-individual cortisol reactivity across the two study challenges. Future research that includes a larger sample and permits more complex statistical investigations, should examine how internalizing behaviour problems relate to cortisol flexibility, assessed using differentially potent challenges. This study should also be replicated with a larger sample size to determine the reliability and generalizability of findings.

Related to generalizability, this study used a community sample of children who were relatively low risk in terms of their socio-demographic profile (e.g., SES) as well as the severity of their internalizing behaviour problems (the majority of children did not have symptoms in the clinical range). Replication with more at-risk and clinical samples is necessary in order to generalize findings. In addition, replication with more sample variability (i.e., including low risk, at at-risk, and clinical samples) may help elucidate these associations, given the potential for stronger effects in samples with greater variability. 
In addition, I was unable to contact 42 families from the original study sample, and thus the sample for the current study may not be representative of the original sample or the population it represents (Atkinson et al., 2013). However, the present subsample did not significantly differ on any demographic variables.

An additional limitation of this study is that a total of 10 children terminated the stressor paradigm prematurely. Although I did not find significant differences in cortisol responsivity between those who completed the challenges and those who asked to terminate early, this may nonetheless confound the study design and findings. Interesting, no other studies using children mention whether participants requested discontinuation of the stressor prematurely. In order to ensure the study was ethical, all children in the present study were told they had the right to discontinue at any time. Given these ethical considerations are ubiquitous, it is unclear whether other studies did not report having children who choose to terminate the stressor early.

Finally, although a strength of this study was the use of both parent and child-reported internalizing behaviours, the specific type of measures used may have complicated the interpretation of findings. That is, the parent-reported CBCL Internalizing scale is a broadband measure of internalizing problems that includes depression, withdrawal, anxiety and somatic symptoms, whereas the childreported depression inventory is an exclusive measure of depressive symptoms. Future research should compare effects of self- and parent-reported internalizing behaviour problems, as well as specific subcomponents, including anxiety, depression, somatic symptoms, in order to completely disentangle these associations. In future work, it will be fruitful to have both parents and children report on broadband measures (e.g., YSR and CBCL) as well as specific symptom measures (e.g., parent and child versions of CDI) to tease apart whether differences are related to the reporter or the type of symptom.

\section{Conclusion}


The present findings indicate that internalizing behaviours are associated with cortisol reactivity and the coordination of cortisol and sAA, however findings differ depending on the stressor paradigm and the reporter (mother or child) of internalizing behaviour problems. In the potent TSST-C, children with higher self-reported depressive symptoms experienced a decline in cortisol across time, however those with lower depressive symptoms showed a robust cortisol increase and return to baseline. In contrast, self-reported depressive symptoms were not associated with cortisol reactivity in the less potent competition challenge. In addition, maternal-reported internalizing behaviours were associated with cortisol-sAA coordination in the competition challenge, such that those with higher internalizing behaviour problems who experienced higher sAA also experienced higher cortisol in this non-potent challenge. No significant associations between maternal-reported internalizing behaviours and cortisol or cortisol-sAA coordination were found in the TSST-C. Taken together, these results suggest that internalizing behaviours are associated with child cortisol reactivity, but this association may be dependent on the stressor paradigm used, coordination with sAA, and the reporter of internalizing behaviour. These findings point to the complexity of the association between internalizing behaviour problems and stress physiology, indicating that future work is needed to truly understand these nuanced associations in order to decipher precisely how stress physiology is associated with internalizing problems. Further, given that these relations are present in middle-childhood, this work suggests that early stress regulation may have the potential to influence future risk for adverse biological and psychological development. 


\section{Appendix 1}

\section{Trier Social Stress Test -Child Version: Story}

Yesterday my best friend Robert and I went home from school. Suddenly, we had the idea to visit Mr. Greg who lived in the big old house located in the dark forest near our town. Mr. Greg was a crazy old man and our parents didn't like the idea that we sometimes went visiting him. There was a rumor in town that there was a mystery about the old house. When we arrived at the house we were surprised that the door was open. Suddenly we heard a strange noise and cautiously, we entered the dark hall. ... 
Appendix 2

Competition Challenge - Star shape for tracing

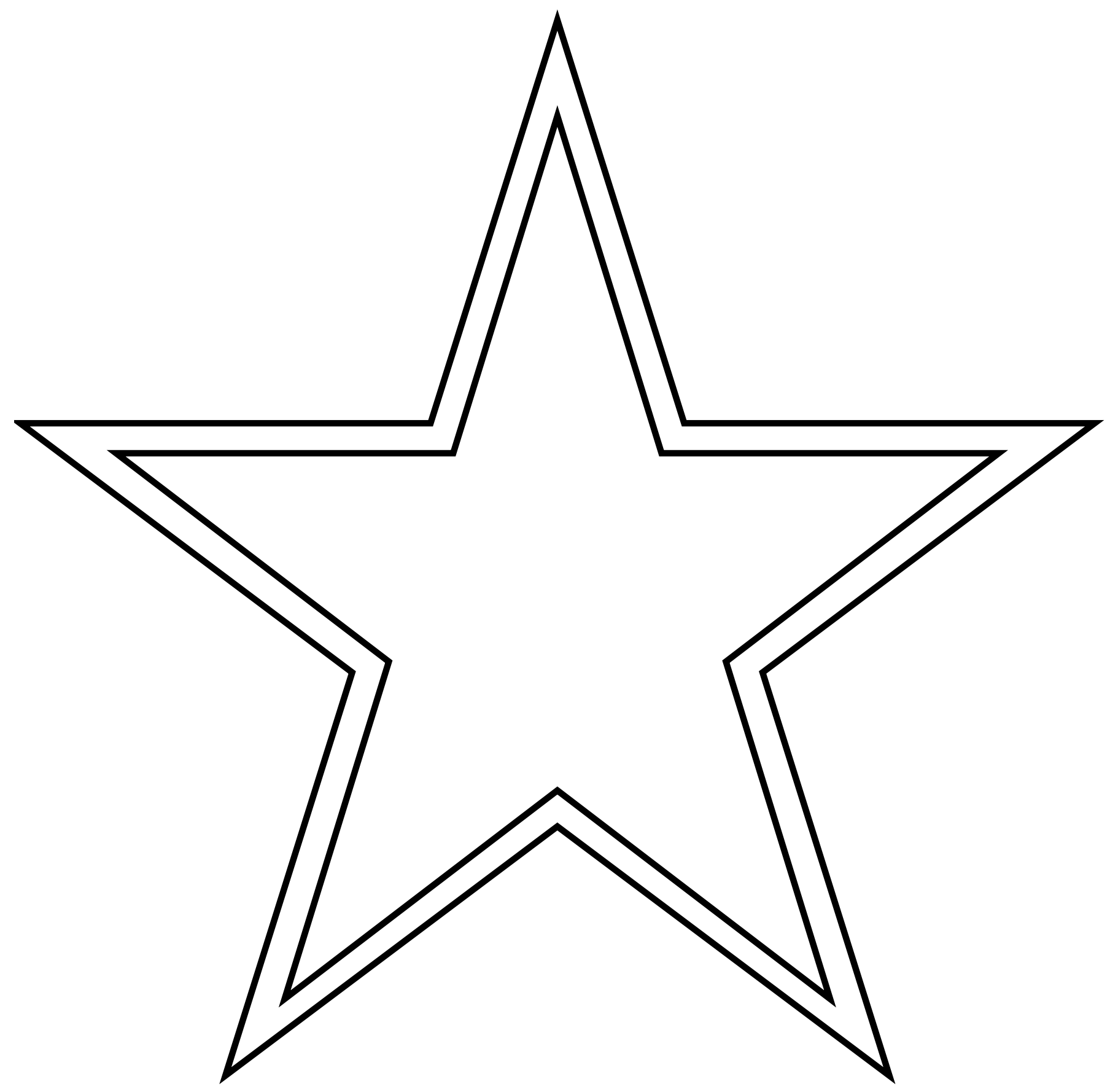


Competition Challenge - Mirror tracing apparatus

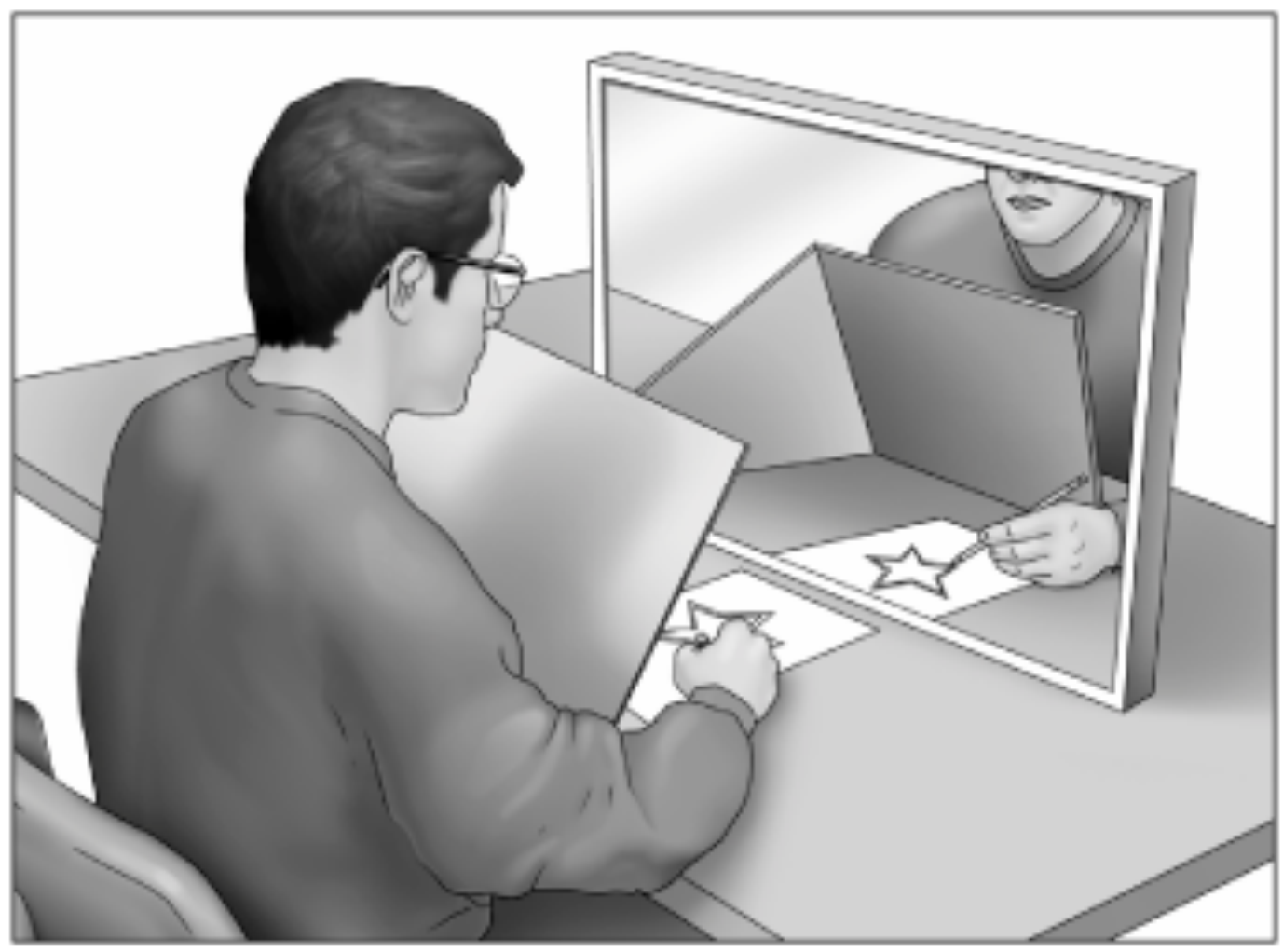


Appendix 3

\section{Competition Challenge - Puzzle}

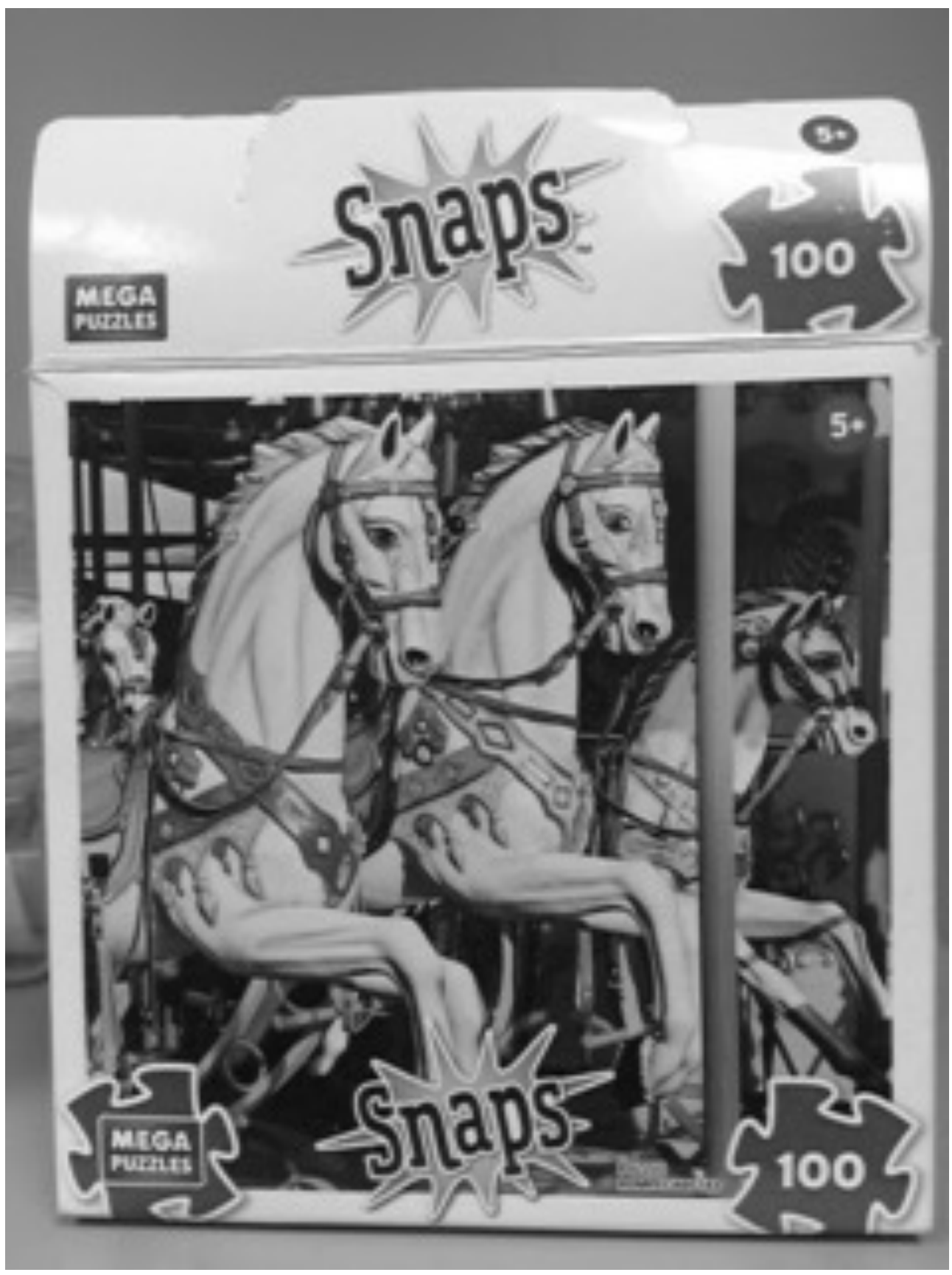




\section{Appendix 4.1}

\section{Model 1:}

\section{Model Equations and Results: Self-Reported Depression in the TSST}

Level-1 Model

$$
\text { LG_CORT }=\mathrm{B} 0+\mathrm{r}
$$

Level-2 Model

$$
\mathrm{B} 0=\mathrm{G} 00+\mathrm{u} 0
$$

Mixed Model

$$
\text { LG_CORT }=\mathrm{G} 00+\mathrm{u} 0+\mathrm{r}
$$

$\operatorname{sigma}^{\wedge} 2=0.04527$

Standard error of sigma^ ${ }^{\wedge}=0.00454$

tau

INTRCPT1,B0 0.05815

Standard error of tau

INTRCPT1,B0 0.01472

Random level-1 coefficient Reliability estimate

INTRCPT1, G0 $\quad 0.885$

The value of the log-likelihood function at iteration $4=-1.154789 \mathrm{E}+001$

\begin{tabular}{|c|c|c|c|c|}
\hline Fixed Effect & $\begin{array}{c}\text { Standard } \\
\text { Coefficient Erro }\end{array}$ & $\begin{array}{r}\text { Approx. } \\
\text { T-ratio }\end{array}$ & d.f. & P-value \\
\hline $\begin{array}{l}\text { For INTRC } \\
\text { INTRCPT2 }\end{array}$ & $\begin{array}{l}\text { 1, B0 } \\
0.581962\end{array}$ & .040537 & 14.356 & 39 \\
\hline
\end{tabular}

The outcome variable is LG_CORT

Final estimation of fixed effects:

The outcome variable is LG_CORT 
Final estimation of variance components:

Random Effect Standard Variance df Chi-square P-value Deviation Component

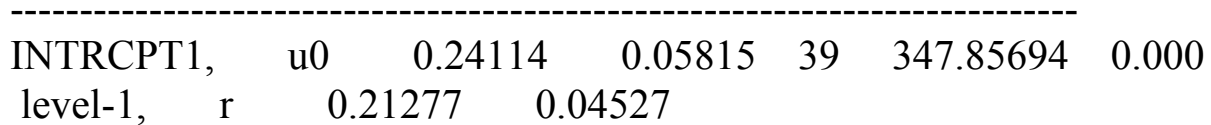

Statistics for the current model

Deviance $\quad=23.095770$

Number of estimated parameters $=3$

\section{Model 2}

Level-1 Model

$$
\text { LG_CORT }=\mathrm{B} 0+\mathrm{B} 1 *(\mathrm{TIME})+\mathrm{r}
$$

Level-2 Model

$$
\begin{aligned}
& \mathrm{B} 0=\mathrm{G} 00+\mathrm{u} 0 \\
& \mathrm{~B} 1=\mathrm{G} 10
\end{aligned}
$$

Mixed Model

LG_CORT $=$ G00

$+\overline{\mathrm{G}} 10 * \mathrm{TIME}+\mathrm{u} 0+\mathrm{r}$

sigma $^{\wedge} 2=0.04505$

Standard error of $\operatorname{sigma}^{\wedge} 2=0.00452$

tau

INTRCPT1,B0 0.05819

Standard error of tau

INTRCPT1,B0 0.01472

Random level-1 coefficient Reliability estimate

INTRCPT1, G0 $\quad 0.885$ 
The value of the log-likelihood function at iteration $4=-1.105267 \mathrm{E}+001$

The outcome variable is LG_CORT

Final estimation of fixed effects:

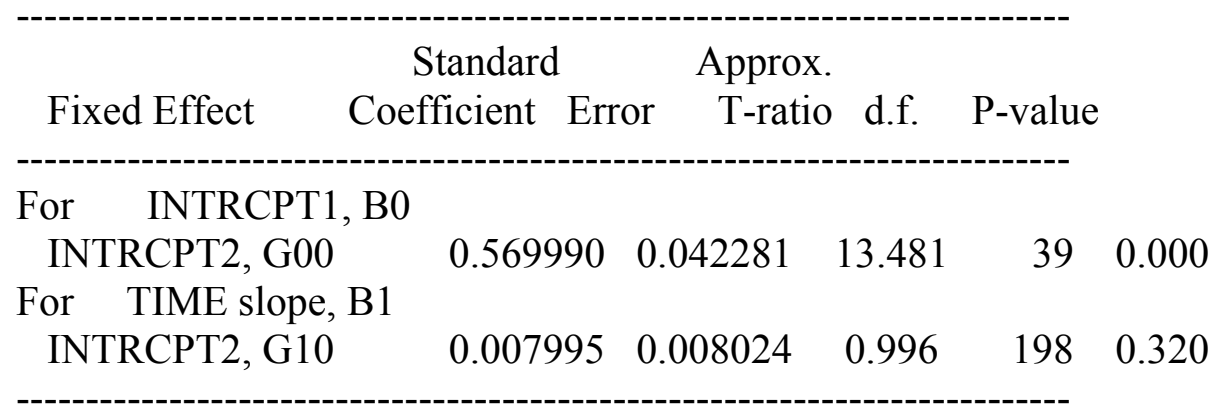

The outcome variable is LG_CORT

Final estimation of variance components:

Random Effect Standard Variance df Chi-square P-value Deviation Component

$\begin{array}{llllll}\begin{array}{l}\text { INTRCPT1, } \\ \text { level-1, ro }\end{array} \quad \text { r } 0.24122 & 0.05819 & 39 & 349.61705 & 0.000\end{array}$

Statistics for the current model

Deviance $\quad=22.105337$

Number of estimated parameters $=4$

Model comparison test

Chi-square statistic $=0.99043$
Degrees of freedom $=1$
P-value $=>.500$

\section{Model 3}

Level-1 Model

$$
\text { LG_CORT }=\mathrm{B} 0+\mathrm{B} 1 *(\mathrm{TIME})+\mathrm{B} 2 *\left(\mathrm{QUAD} \_\mathrm{TIM}\right)+\mathrm{r}
$$


Level-2 Model

$$
\begin{aligned}
& \mathrm{B} 0=\mathrm{G} 00+\mathrm{u} 0 \\
& \mathrm{~B} 1=\mathrm{G} 10 \\
& \mathrm{~B} 2=\mathrm{G} 20
\end{aligned}
$$

$$
\begin{aligned}
& \text { Mixed Model } \\
& \text { LG_CORT }=\text { G00 } \\
& + \text { G10*TIME } \\
& + \text { G20*QUAD_TIM }+\mathrm{u} 0+\mathrm{r}
\end{aligned}
$$

$\operatorname{sigma}^{\wedge} 2=0.04258$

Standard error of sigma^ $2=0.00427$

tau

INTRCPT1,B0 0.05865

Standard error of tau

INTRCPT1,B0 0.01473

Random level-1 coefficient Reliability estimate

INTRCPT1, G0 $\quad 0.892$

The value of the log-likelihood function at iteration $4=-5.464535 \mathrm{E}+000$

The outcome variable is LG_CORT

Final estimation of fixed effects:

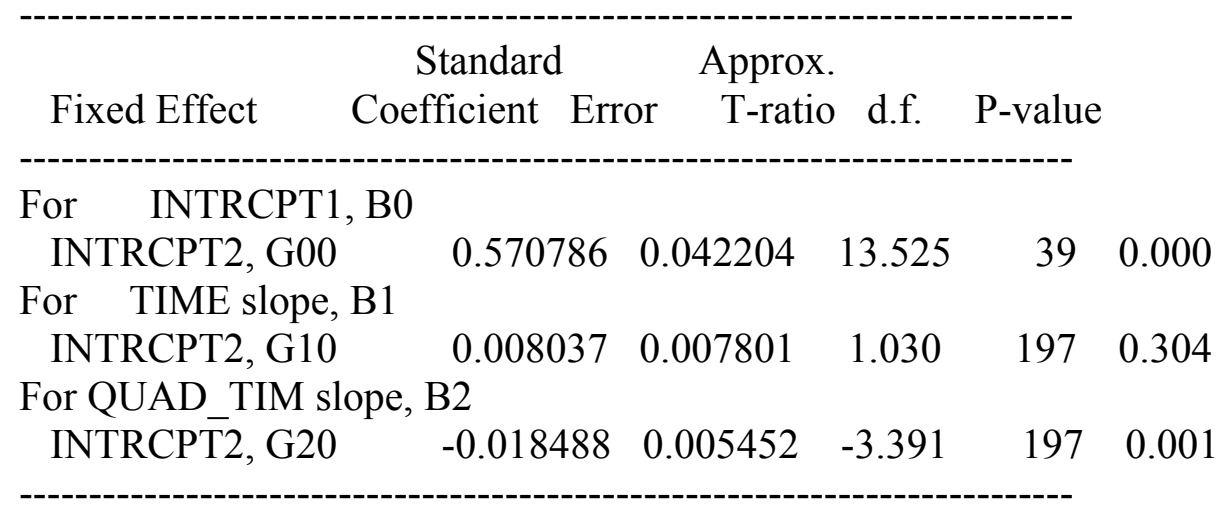

The outcome variable is LG_CORT 
Final estimation of variance components:

Random Effect Standard Variance df Chi-square P-value
Deviation Component

$\begin{array}{lllllll}\text { INTRCPT1, u0 } & 0.24219 & 0.05865 & 39 & 370.14487 & 0.000\end{array}$

$\begin{array}{llll}\text { level-1, } & \mathrm{r} & 0.20635 & 0.04258\end{array}$

Statistics for the current model

Deviance $\quad=10.929069$

Number of estimated parameters $=5$

Model comparison test

Chi-square statistic $=11.17627$

Degrees of freedom $=1$

$\mathrm{P}$-value $\quad=0.001$

\section{Model 4}

Level-1 Model

$$
\text { LG_CORT }=\text { B0 + B1 } *(\text { TIME })+\text { B2 } *\left(Q U A D \_T I M\right)+B 3 *\left(L G \_S A A\right)+r
$$

Level-2 Model

$$
\begin{aligned}
& \mathrm{B} 0=\mathrm{G} 00+\mathrm{u} 0 \\
& \mathrm{~B} 1=\mathrm{G} 10 \\
& \mathrm{~B} 2=\mathrm{G} 20 \\
& \mathrm{~B} 3=\mathrm{G} 30
\end{aligned}
$$

LG_SAA has been centered around the group mean.

$$
\begin{aligned}
& \text { Mixed Model } \\
& \text { LG_CORT }=\text { G00 } \\
& + \text { G10*TIME } \\
& + \text { G20*QUAD_TIM } \\
& + \text { G30*LG_SAA } \\
& +\mathrm{u} 0+\mathrm{r}
\end{aligned}
$$

$\operatorname{sigma}^{\wedge} 2=0.04378$ 
Standard error of sigma^ ${ }^{\wedge}=0.00520$

tau

INTRCPT1,B0 0.06191

Standard error of tau

INTRCPT1,B0 0.01672

Random level-1 coefficient Reliability estimate

INTRCPT1, G0 $\quad 0.875$

The value of the log-likelihood function at iteration $5=-1.152421 \mathrm{E}+001$

The outcome variable is LG_CORT

Final estimation of fixed effects:

\begin{tabular}{|c|c|c|c|c|c|}
\hline Fixed Effect & $\begin{array}{c}\text { Standard } \\
\text { Coefficient Err }\end{array}$ & $\begin{array}{r}\text { Approx } \\
\text { or } \quad \text { T-ratio }\end{array}$ & d.f. & P-value & \\
\hline \multicolumn{6}{|c|}{ For INTRCPT1, B0 } \\
\hline INTRCPT2, G00 & 0.574991 & 0.045890 & 12.530 & 35 & 0.000 \\
\hline \multicolumn{6}{|c|}{ For TIME slope, B1 } \\
\hline INTRCPT2, G10 & 0.021358 & 0.014592 & 1.464 & 139 & 0.146 \\
\hline \multicolumn{6}{|c|}{ For QUAD_TIM slope, B2 } \\
\hline INTRCPT2, G20 & -0.014418 & 0.009612 & -1.500 & 139 & 0.13 \\
\hline \multicolumn{6}{|c|}{ For LG_SAA slope, B3 } \\
\hline INTRCPT2, G30 & 0.253683 & 0.129530 & 1.958 & 139 & 0.052 \\
\hline
\end{tabular}

The outcome variable is LG_CORT

Final estimation of variance components:

Random Effect Standard Variance df Chi-square P-value Deviation Component

$\begin{array}{lllllll}\text { INTRCPT1, } \quad \mathrm{u} 0 & 0.24882 & 0.06191 & 35 & 289.72802 & 0.000\end{array}$
level-1, r $\quad 0.20924 \quad 0.04378$ 
Statistics for the current model

Deviance $\quad=23.048416$

Number of estimated parameters $=6$

Model comparison test

Chi-square statistic $=12.11935$

Degrees of freedom $=1$

$\mathrm{P}$-value $\quad=\quad<0.001$

\section{Model 5}

Level-1 Model

$$
\text { LG_CORT }=\text { B0 }+ \text { B1*(TIME })+ \text { B2 } *\left(Q U A D \_T I M\right)+B 3 *\left(L G \_S A A\right)+B 4 *(S A A X T I M E)+r
$$

Level-2 Model

$$
\begin{aligned}
& \mathrm{B} 0=\mathrm{G} 00+\mathrm{u} 0 \\
& \mathrm{~B} 1=\mathrm{G} 10 \\
& \mathrm{~B} 2=\mathrm{G} 20 \\
& \mathrm{~B} 3=\mathrm{G} 30 \\
& \mathrm{~B} 4=\mathrm{G} 40
\end{aligned}
$$

LG_SAA has been centered around the group mean.

$$
\begin{aligned}
& \text { Mixed Model } \\
& \text { LG_CORT = G00 } \\
& \text { + G10*TIME } \\
& \text { + G20*QUAD_TIM } \\
& + \text { G30*LG_SAA } \\
& \text { + G40*SAAXTIME } \\
& +\mathrm{u} 0+\mathrm{r}
\end{aligned}
$$

$\operatorname{sigma}^{\wedge} 2=0.04350$

Standard error of $\operatorname{sigma}^{\wedge} 2=0.00516$

tau

INTRCPT1,B0 0.06061

Standard error of tau

INTRCPT1,B0 0.01640 
Random level-1 coefficient Reliability estimate

INTRCPT1, G0 $\quad 0.873$

The value of the log-likelihood function at iteration $7=-1.071677 \mathrm{E}+001$

The outcome variable is LG_CORT

Final estimation of fixed effects:

\begin{tabular}{|c|c|c|c|c|c|}
\hline Fixed Effect & $\begin{array}{c}\text { Standard } \\
\text { Coefficient Erro }\end{array}$ & $\begin{array}{r}\text { Approx. } \\
\text { T-ratio }\end{array}$ & d.f. & P-value & \\
\hline \multicolumn{6}{|c|}{ For INTRCPT1, B0 } \\
\hline \multicolumn{6}{|c|}{ For TIME slope, B1 } \\
\hline INTRCPT2, G & 0.024608 & 0.014766 & 1.666 & 138 & 0.098 \\
\hline \multicolumn{6}{|c|}{ For QUAD TIM slope, B2 } \\
\hline INTRCPT2, G & -0.013325 & 0.009619 & -1.385 & 138 & 0.168 \\
\hline \multicolumn{6}{|c|}{ For LG SAA slope, B3 } \\
\hline \multicolumn{6}{|c|}{ For SAAXTIME slope, B4 } \\
\hline INTRCPT2, G & -0.102169 & 0.080155 & -1.275 & 138 & 0.205 \\
\hline
\end{tabular}

The outcome variable is LG_CORT

Final estimation of variance components:

Random Effect Standard Variance df Chi-square P-value Deviation Component

$\begin{array}{lllllll} & & & & \\ \text { INTRCPT1, } & \text { u0 } & 0.24618 & 0.06061 & 35 & 286.02241 & 0.000\end{array}$

$\begin{array}{llll}\text { level-1, } & \mathrm{r} & 0.20856 & 0.04350\end{array}$

Statistics for the current model

Deviance $\quad=21.433540$

Number of estimated parameters $=7$

Model comparison test 
Chi-square statistic $=1.61488$

Degrees of freedom $=1$

P-value $\quad=0.201$

\section{Model 6}

Level-1 Model

LG_CORT $=\mathrm{B} 0+\mathrm{B} 1 *(\mathrm{TIME})+\mathrm{B} 2 *\left(\mathrm{QUAD} \_\mathrm{TIM}\right)+\mathrm{B} 3 *\left(\mathrm{LG}_{-} \mathrm{SAA}\right)+\mathrm{B} 4 *(\mathrm{SAAXTIME})+$ $\mathrm{B} 5 *(\mathrm{SAAXQ}$ TI $)+\mathrm{r}$

Level-2 Model

$$
\begin{aligned}
& \mathrm{B} 0=\mathrm{G} 00+\mathrm{u} 0 \\
& \mathrm{~B} 1=\mathrm{G} 10 \\
& \mathrm{~B} 2=\mathrm{G} 20 \\
& \mathrm{~B} 3=\mathrm{G} 30 \\
& \mathrm{~B} 4=\mathrm{G} 40 \\
& \mathrm{~B} 5=\mathrm{G} 50
\end{aligned}
$$

LG_SAA has been centered around the group mean.

$$
\begin{aligned}
& \text { Mixed Model } \\
& \text { LG_CORT }=\text { G00 } \\
& + \text { G10*TIME } \\
& \text { + G20*QUAD_TIM } \\
& \text { + G30*LG_SAA } \\
& \text { + G40*SAAXTIME } \\
& \text { + G50*SAAXQ_TI } \\
& \text { + } 0+\mathrm{r}
\end{aligned}
$$

$\operatorname{sigma}^{\wedge} 2=0.04339$

Standard error of $\operatorname{sigma}^{\wedge} 2=0.00515$

tau

INTRCPT1,B0 0.06030

Standard error of tau

INTRCPT1,B0 0.01632

Random level-1 coefficient Reliability estimate

INTRCPT1, G0 $\quad 0.873$ 
The value of the log-likelihood function at iteration $7=-1.044688 \mathrm{E}+001$

The outcome variable is LG_CORT

Final estimation of fixed effects:

\begin{tabular}{|c|c|c|c|c|c|}
\hline Fixed Effect & $\begin{array}{c}\text { Standard } \\
\text { Coefficient Erro }\end{array}$ & $\begin{array}{r}\text { Approx. } \\
\text { or } \quad \text { T-ratio }\end{array}$ & d.f. & P-value & \\
\hline \multicolumn{6}{|c|}{ For INTRCPT1, B0 } \\
\hline INTRCPT2, G00 & 0.574178 & 0.045364 & 12.657 & 35 & 0.000 \\
\hline \multicolumn{6}{|c|}{ For TIME slope, B1 } \\
\hline INTRCPT2, G10 & 0.023018 & 0.014905 & 1.544 & 137 & 0.125 \\
\hline \multicolumn{6}{|c|}{ For QUAD_TIM slope, B2 } \\
\hline INTRCPT̄2, G20 & -0.013869 & 0.009635 & -1.439 & 137 & 0.152 \\
\hline \multicolumn{6}{|c|}{ For LG_SAA slope, B3 } \\
\hline INTRCPT2, G30 & 0.190863 & 0.144380 & 1.322 & 137 & 0.188 \\
\hline \multicolumn{6}{|c|}{ For SAAXTIME slope, B4 } \\
\hline INTRCPT2, G40 & -0.036269 & 0.120208 & -0.302 & 137 & 0.763 \\
\hline \multicolumn{6}{|c|}{ For SAAXQ_TI slope, B5 } \\
\hline INTRCPT2, G50 & 0.048619 & 0.066122 & 0.735 & 137 & 0.463 \\
\hline
\end{tabular}

The outcome variable is LG_CORT

Final estimation of variance components:

Random Effect Standard Variance df Chi-square P-value Deviation Component

$\begin{array}{lllllll}\text { INTRCPT1， u0 } & 0.24555 & 0.06030 & 35 & 285.37308 & 0.000\end{array}$ $\begin{array}{llll}\text { level-1, } & \mathrm{r} & 0.20830 & 0.04339\end{array}$

Statistics for the current model

Deviance $\quad=20.893763$

Number of estimated parameters $=8$

Model comparison test

Chi-square statistic $=0.53978$

Degrees of freedom $=1$ 
P-value $\quad=\quad>.500$

\section{Model 7}

Level-1 Model

$\mathrm{LG}$ CORT $=\mathrm{B} 0+\mathrm{B} 1 *(\mathrm{TIME})+\mathrm{B} 2 *(\mathrm{QUAD}$ TIM $)+\mathrm{B} 3 *(\mathrm{LG}$ _SAA $)+\mathrm{B} 4 *(\mathrm{SAAXTIME})+$ $\mathrm{B} 5 *(\mathrm{SAAX} Q \mathrm{TI})+\mathrm{r}$

Level-2 Model

$$
\begin{aligned}
& \mathrm{B} 0=\mathrm{G} 00+\mathrm{u} 0 \\
& \mathrm{~B} 1=\mathrm{G} 10+\mathrm{u} 1 \\
& \mathrm{~B} 2=\mathrm{G} 20+\mathrm{u} 2 \\
& \mathrm{~B} 3=\mathrm{G} 30 \\
& \mathrm{~B} 4=\mathrm{G} 40 \\
& \mathrm{~B} 5=\mathrm{G} 50
\end{aligned}
$$

LG_SAA has been centered around the group mean.

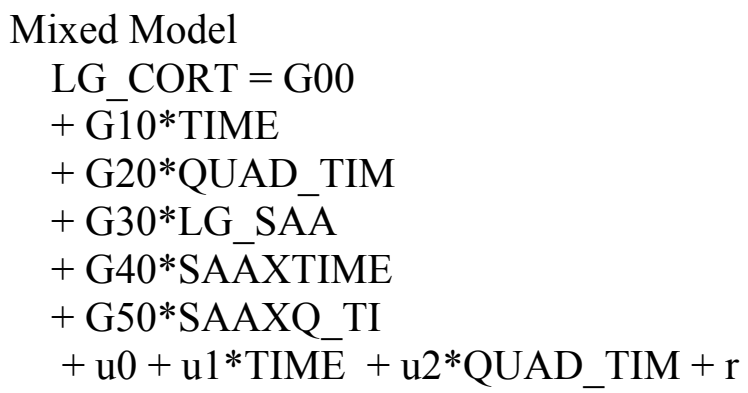

$\operatorname{sigma}^{\wedge} 2=0.02301$

Standard error of $\operatorname{sigma}^{\wedge} 2=\quad 0.00387$

tau

$\begin{array}{cccc}\text { INTRCPT1,B0 } & 0.05426 & -0.00050 & -0.00562 \\ \text { TIME,B1 } & -0.00050 & 0.00178 & -0.00136 \\ \text { QUAD_TIM,B2 } & -0.00562 & -0.00136 & 0.00171\end{array}$

Standard errors of tau

$\begin{array}{cccc}\text { INTRCPT1,B0 } & 0.01457 & 0.00322 & 0.00264 \\ \text { TIME,B1 } & 0.00322 & 0.00150 & 0.00079 \\ \text { QUAD_TIM,B2 } & 0.00264 & 0.00079 & 0.00086\end{array}$

tau (as correlations)

INTRCPT1,B0 $1.000-0.051-0.583$ 
TIME,B1 -0.051 $1.000-0.780$

QUAD_TIM,B2 $-0.583-0.7801 .000$

Random level-1 coefficient Reliability estimate

\begin{tabular}{cc} 
INTRCPT1, G0 & 0.876 \\
TIME, G1 & 0.310 \\
QUAD_TIM, G2 & 0.492 \\
\hline
\end{tabular}

The outcome variable is LG_CORT

Final estimation of fixed effects:

\begin{tabular}{|c|c|c|c|c|c|}
\hline Fixed Effect & $\begin{array}{c}\text { Standard } \\
\text { Coefficient Erro }\end{array}$ & $\begin{array}{r}\text { Approx. } \\
\text { or } \quad \text { T-ratio }\end{array}$ & d.f. & P-value & \\
\hline \multicolumn{3}{|c|}{ For INTRCPT1, B0 } & & & 0.000 \\
\hline $\begin{array}{l}\text { For TIME slope, } \\
\text { INTRCPT2, G10 }\end{array}$ & B1 & 0.01 & 424 & 35 & 0.163 \\
\hline \multicolumn{6}{|c|}{ For QUAD_TIM slope, B2 } \\
\hline \multicolumn{6}{|c|}{ For LG_SAA slope, B3 } \\
\hline INTRCPT2, G30 & 0.023501 & 0.135326 & 0.174 & 67 & 0.863 \\
\hline \multicolumn{6}{|c|}{ For SAAXTIME slope, B4 } \\
\hline INTRCPT2, G40 & -0.000986 & 0.096991 & -0.010 & 67 & 0.992 \\
\hline \multicolumn{6}{|c|}{ For SAAXQ TI slope, B5 } \\
\hline INTRCPT2, G50 & 0.065483 & 0.054359 & 1.205 & 67 & 0.233 \\
\hline
\end{tabular}

The outcome variable is LG_CORT

Final estimation of variance components:

Random Effect Standard Variance df Chi-square P-value Deviation Component

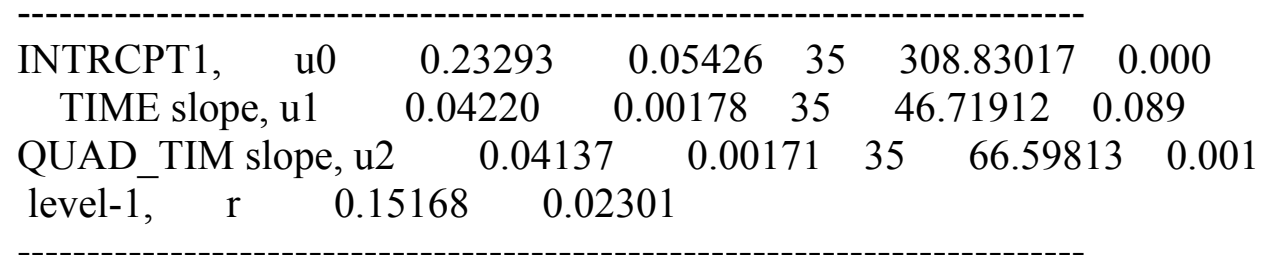


Statistics for the current model

Deviance $\quad=-24.902481$

Number of estimated parameters $=13$

Model comparison test

Chi-square statistic $=45.79624$

Degrees of freedom $=5$

P-value $\quad=\quad<0.001$

\section{Model 8}

Level-1 Model

LG_CORT $=$ B0 + B1* $($ TIME $)+$ B2* $\left(\mathrm{QUAD} \_T I M\right)+B 3 *\left(L G \_S A A\right)+B 4 *($ SAAXTIME $)+$ $\mathrm{B} 5 *\left(\mathrm{SAAX} \mathrm{Q}_{-} \mathrm{TI}\right)+\mathrm{r}$

Level-2 Model

$$
\begin{aligned}
& \mathrm{B} 0=\mathrm{G} 00+\mathrm{u} 0 \\
& \mathrm{~B} 1=\mathrm{G} 10 \\
& \mathrm{~B} 2=\mathrm{G} 20+\mathrm{u} 2 \\
& \mathrm{~B} 3=\mathrm{G} 30+\mathrm{u} 3 \\
& \mathrm{~B} 4=\mathrm{G} 40 \\
& \mathrm{~B} 5=\mathrm{G} 50
\end{aligned}
$$

LG_SAA has been centered around the group mean.

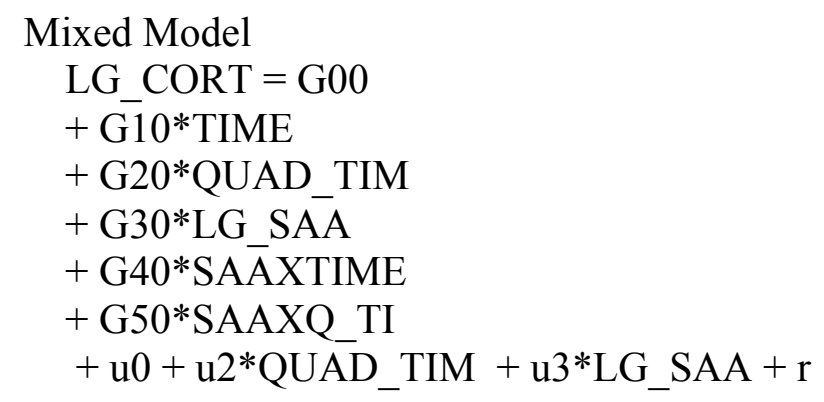

$\operatorname{sigma}^{\wedge} 2=0.02610$

Standard error of $\operatorname{sigma}^{\wedge} 2=0.00383$

tau

$\begin{array}{llll}\text { INTRCPT1,B0 } & 0.05567 & -0.00567 & 0.05148\end{array}$

QUAD_TIM,B2 $-0.00567 \quad 0.00246 \quad 0.00106$ 


\section{LG_SAA,B3 $0.05148 \quad 0.00106 \quad 0.06877$}

\begin{tabular}{lccc}
\multicolumn{4}{l}{ Standard errors of tau } \\
INTRCPT1,B0 & 0.01452 & 0.00263 & 0.03051 \\
QUAD_TIM,B2 & 0.00263 & 0.00087 & 0.00684 \\
LG_SAA,B3 & 0.03051 & 0.00684 & 0.09499
\end{tabular}

tau (as correlations)

INTRCPT1,B0 $1.000-0.484 \quad 0.832$

QUAD_TIM,B2 -0.484 $1.000 \quad 0.082$

LG_SAA,B3 0.8320 .0821 .000

Random level-1 coefficient Reliability estimate

$\begin{array}{lc}\text { INTRCPT1, G0 } & 0.897 \\ \text { QUAD_TIM, G2 } & 0.586 \\ \text { LG_SAA, G3 } & 0.110\end{array}$

The outcome variable is LG_CORT

Final estimation of fixed effects:

\begin{tabular}{|c|c|c|c|c|c|}
\hline Fixed Effect & $\begin{array}{c}\text { Standard } \\
\text { Coefficient Erro }\end{array}$ & $\begin{array}{r}\text { Approx. } \\
\text { T-ratio }\end{array}$ & d.f. & P-value & \\
\hline \multicolumn{6}{|c|}{ For $\quad$ INTRCPT1, B0 } \\
\hline INTRCPT2, G00 & 0.579201 & 0.042204 & 13.724 & 35 & 0.000 \\
\hline \multicolumn{6}{|c|}{ For TIME slope, B1 } \\
\hline INTRCPT2, G10 & 0.018162 & 0.011711 & 1.551 & 67 & 0.126 \\
\hline \multicolumn{6}{|c|}{ For QUAD_TIM slope, B2 } \\
\hline INTRCPT̄ 2, G20 & -0.014479 & 0.011209 & -1.292 & 35 & 0.205 \\
\hline \multicolumn{6}{|c|}{ For LG_SAA slope, B3 } \\
\hline INTRCPT2, G30 & 0.033248 & 0.138032 & 0.241 & 35 & 0.811 \\
\hline \multicolumn{6}{|c|}{ For SAAXTIME slope, B4 } \\
\hline INTRCPT2, G40 & -0.020239 & 0.096141 & -0.211 & 67 & 0.834 \\
\hline \multicolumn{6}{|c|}{ For SAAXQ_TI slope, B5 } \\
\hline INTRCPT2, G50 & 0.061312 & 0.058838 & 1.042 & 67 & 0.301 \\
\hline
\end{tabular}

The outcome variable is LG_CORT

Final estimation of variance components:

Random Effect Standard Variance df Chi-square P-value 
Deviation Component

$\begin{array}{lllllll}\text { INTRCPT1， u0 } & 0.23595 & 0.05567 & 35 & 343.33414 & 0.000\end{array}$

QUAD_TIM slope, u2 $\quad 0.04964 \quad 0.00246 \quad 35 \quad 84.54331 \quad 0.000$

$\begin{array}{llllll}\text { LG_SAA slope, u3 } & 0.26225 & 0.06877 & 35 & 26.12914 & >.500\end{array}$

$\begin{array}{llll}\text { level-1, } & \mathrm{r} & 0.16154 & 0.02610\end{array}$

Statistics for the current model

Deviance $\quad=-17.717672$

Number of estimated parameters $=13$

Model comparison test

Chi-square statistic $=38.61144$

Degrees of freedom $=5$

$\mathrm{P}$-value $\quad=\quad<0.001$

\section{Model 9}

Level-1 Model

LG_CORT $=$ B0 + B $1 *($ TIME $)+$ B2 $*\left(Q U A D \_T I M\right)+B 3 *\left(L G \_S A A\right)+B 4 *($ SAAXTIME $)+$ $\mathrm{B} 5 *(\mathrm{SAAXQ} \mathrm{TI})+\mathrm{r}$

Level-2 Model

$$
\begin{aligned}
& \mathrm{B} 0=\mathrm{G} 00+\mathrm{u} 0 \\
& \mathrm{~B} 1=\mathrm{G} 10 \\
& \mathrm{~B} 2=\mathrm{G} 20+\mathrm{u} 2 \\
& \mathrm{~B} 3=\mathrm{G} 30 \\
& \mathrm{~B} 4=\mathrm{G} 40 \\
& \mathrm{~B} 5=\mathrm{G} 50
\end{aligned}
$$

LG_SAA has been centered around the group mean.

$$
\begin{aligned}
& \text { Mixed Model } \\
& \text { LG_CORT = G00 } \\
& + \text { +G10*TIME } \\
& + \text { G20*QUAD_TIM } \\
& \text { + G30*LG_SAA } \\
& \text { + G40*SAAXTIME } \\
& \text { + G50*SAAXQ_TI }
\end{aligned}
$$




$$
+\mathrm{u} 0+\mathrm{u} 2 * \mathrm{QUAD} \_\mathrm{TIM}+\mathrm{r}
$$

$\operatorname{sigma}^{\wedge} 2=0.02685$

Standard error of sigma^2 $=0.00368$

tau

INTRCPT1,B0 $0.05347 \quad-0.00709$

QUAD_TIM,B2 $-0.00709 \quad 0.00296$

Standard errors of tau

$\begin{array}{lll}\text { INTRCPT1,B0 } & 0.01403 & 0.00283\end{array}$

$\begin{array}{lll}\text { QUAD_TIM,B2 } & 0.00283 & 0.00099\end{array}$

tau (as correlations)

INTRCPT1,B0 $1.000-0.563$

QUAD_TIM,B2 -0.563 1.000

Random level-1 coefficient Reliability estimate

$\begin{array}{lc}\text { INTRCPT1, G0 } & 0.896 \\ \text { QUAD_TIM, G2 } & 0.706\end{array}$

The value of the log-likelihood function at iteration $12=7.103355 \mathrm{E}+000$

The outcome variable is LG_CORT

Final estimation of fixed effects:

\begin{tabular}{|c|c|c|c|c|c|}
\hline Fixed Effect & $\begin{array}{c}\text { Standard } \\
\text { Coefficient Erro }\end{array}$ & $\begin{array}{r}\text { Approx. } \\
\text { or } \quad \text { T-ratio }\end{array}$ & d.f. & P-value & \\
\hline \multicolumn{6}{|c|}{ For INTRCPT1, B0 } \\
\hline INTRCPT2, G00 & 0.577792 & 0.041535 & 13.911 & 35 & 0.000 \\
\hline \multicolumn{6}{|c|}{ For TIME slope, B1 } \\
\hline INTRCPT2, G10 & 0.019582 & 0.011791 & 1.661 & 102 & 0.100 \\
\hline \multicolumn{6}{|c|}{ For QUAD_TIM slope, B2 } \\
\hline INTRCPT̄2, G20 & -0.015151 & 0.011855 & -1.278 & 35 & 0.210 \\
\hline \multicolumn{6}{|c|}{ For LG_SAA slope, B3 } \\
\hline INTRCPT2, G30 & 0.013329 & 0.132117 & 0.101 & 102 & 0.920 \\
\hline \multicolumn{6}{|c|}{ For SAAXTIME slope, B4 } \\
\hline INTRCPT2, G40 & -0.030696 & 0.097449 & -0.315 & 102 & 0.753 \\
\hline \multicolumn{6}{|c|}{ For SAAXQ_TI slope, B5 } \\
\hline INTRCPT2, G50 & 0.057450 & 0.059370 & 0.968 & 102 & 0.336 \\
\hline
\end{tabular}


The outcome variable is LG_CORT

Final estimation of variance components:

Random Effect Standard Variance df Chi-square P-value
Deviation Component

$\begin{array}{lllllll}\text { INTRCPT1, u0 } & 0.23124 & 0.05347 & 35 & 365.69491 & 0.000 \\ \text { QUAD_TIM slope, } \mathrm{u} 2 & 0.05443 & 0.00296 & 35 & 126.62201 & 0.000 \\ \text { level-1, r } & 0.16387 & 0.02685 & & & & \end{array}$

Statistics for the current model

Deviance $\quad=-14.206709$

Number of estimated parameters $=10$

Model comparison test

Chi-square statistic $=35.10047$

Degrees of freedom $=2$

$\mathrm{P}$-value $\quad=\quad<0.001$

\section{Model 10}

Level-1 Model

LG_CORT $=$ B0 + B1 $*($ TIME $)+$ B2 $*\left(Q U A D \_T I M\right)+B 3 *\left(L G \_S A A\right)+$ B4 $*($ SAAXTIME $)+$ $\mathrm{B} 5 *(\mathrm{SAAXQ}$ TI $)+\mathrm{r}$

Level-2 Model

$$
\begin{aligned}
& \mathrm{B} 0=\mathrm{G} 00+\mathrm{G} 01 *(\mathrm{CDI} \text { TOTA })+\mathrm{u} 0 \\
& \mathrm{~B} 1=\mathrm{G} 10 \\
& \mathrm{~B} 2=\mathrm{G} 20+\mathrm{u} 2 \\
& \mathrm{~B} 3=\mathrm{G} 30 \\
& \mathrm{~B} 4=\mathrm{G} 40 \\
& \mathrm{~B} 5=\mathrm{G} 50
\end{aligned}
$$

LG_SAA has been centered around the group mean. 
CDI_TOTA has been centered around the grand mean.

$$
\begin{aligned}
& \text { Mixed Model } \\
& \text { LG_CORT }=\mathrm{G} 00+\mathrm{G} 01 * \text { CDI_TOTA } \\
& + \text { G10*TIME } \\
& + \text { G } 20 * \text { QUAD_TIM } \\
& \text { + G30*LG_SAA } \\
& \text { + G40*SAAXTIME } \\
& \text { + G50*SAAXQ_TI } \\
& \text { + u0 + u2*QUAD_TIM + r }
\end{aligned}
$$

$\operatorname{sigma}^{\wedge} 2=0.02686$

Standard error of $\operatorname{sigma}^{\wedge} 2=0.00368$

$$
\begin{array}{lcc}
\text { tau } & & \\
\text { INTRCPT1,B0 } & 0.05354 & -0.00711 \\
\text { QUAD_TIM,B2 } & -0.00711 & 0.00296
\end{array}
$$

Standard errors of tau

$$
\begin{array}{lcc}
\text { INTRCPT1,B0 } & 0.01405 & 0.00283 \\
\text { QUAD_TIM,B2 } & 0.00283 & 0.00099
\end{array}
$$

tau (as correlations)

INTRCPT1,B0 $1.000-0.565$

QUAD_TIM,B2 -0.565 1.000

Random level-1 coefficient Reliability estimate

$\begin{array}{lc}\text { INTRCPT1, G0 } & 0.896 \\ \text { QUAD_TIM, G2 } & 0.706\end{array}$

The value of the log-likelihood function at iteration $15=7.103656 \mathrm{E}+000$

The outcome variable is LG_CORT

Final estimation of fixed effects:

\begin{tabular}{lcccrrr} 
& \multicolumn{5}{c}{ Standard } & Approx. \\
Fixed Effect & Coefficient & Error & T-ratio & d.f. & P-value & \\
\hline For INTRCPT1, B0 & & & & & \\
INTRCPT2, G00 & 0.577822 & 0.041578 & 13.897 & 34 & 0.000 \\
CDI_TOTA, G01 & 0.000159 & 0.005980 & 0.027 & 34 & 0.979
\end{tabular}


For TIME slope, B1

$\begin{array}{llllll}\text { INTRCPT2, G10 } & 0.019582 & 0.011792 & 1.661 & 102 & 0.100\end{array}$

For QUAD_TIM slope, B2

$\begin{array}{llllll}\text { INTRCPT2, G20 } & -0.015154 & 0.011855 & -1.278 & 35 & 0.210\end{array}$

For LG_SAA slope, B3

$\begin{array}{llllll}\text { INTRCPT2, G30 } & 0.013277 & 0.132121 & 0.100 & 102 & 0.920\end{array}$

For SAAXTIME slope, B4

$\begin{array}{llllll}\text { INTRCPT2, G40 } & -0.030643 & 0.097455 & -0.314 & 102 & 0.754\end{array}$

For SAAXQ_TI slope, B5

$\begin{array}{llllll}\text { INTRCPT2, G50 } & 0.057533 & 0.059403 & 0.969 & 102 & 0.335\end{array}$

Final estimation of variance components:

Random Effect Standard Variance df Chi-square P-value Deviation Component

$\begin{array}{lllllll}\text { INTRCPT1, u0 } & 0.23140 & 0.05354 & 34 & 366.15520 & 0.000\end{array}$

QUAD_TIM slope, u2 $\quad 0.05442 \quad 0.00296 \quad 35 \quad 126.62145 \quad 0.000$

level-1, $\quad \mathrm{r} \quad 0.16388 \quad 0.02686$

Statistics for the current model

Deviance $\quad=-14.207312$

Number of estimated parameters $=11$

Model comparison test

Chi-square statistic $=0.00060$

Degrees of freedom $=1$

P-value $\quad=\quad>.500$

\section{Model 11}

Level-1 Model

LG_CORT $=$ B0 + B1 $*($ TIME $)+$ B2 $*\left(Q U A D \_T I M\right)+B 3 *\left(L G \_S A A\right)+B 4 *(S A A X T I M E)+$ $\mathrm{B} 5 *(\mathrm{SAAXQ} \mathrm{TI})+\mathrm{r}$

Level-2 Model

$$
\begin{aligned}
& \mathrm{B} 0=\mathrm{G} 00+\mathrm{G} 01 *(\mathrm{CDI} \text { TOTA })+\mathrm{u} 0 \\
& \mathrm{~B} 1=\mathrm{G} 10+\mathrm{G} 11 *(\mathrm{CDI} \text { ITOTA }) \\
& \mathrm{B} 2=\mathrm{G} 20+\mathrm{G} 21 *(\mathrm{CDI} \text { _TOTA })+\mathrm{u} 2
\end{aligned}
$$




$$
\begin{aligned}
& \mathrm{B} 3=\mathrm{G} 30 \\
& \mathrm{~B} 4=\mathrm{G} 40 \\
& \mathrm{~B} 5=\mathrm{G} 50
\end{aligned}
$$

LG_SAA has been centered around the group mean.

CDI_TOTA has been centered around the grand mean.

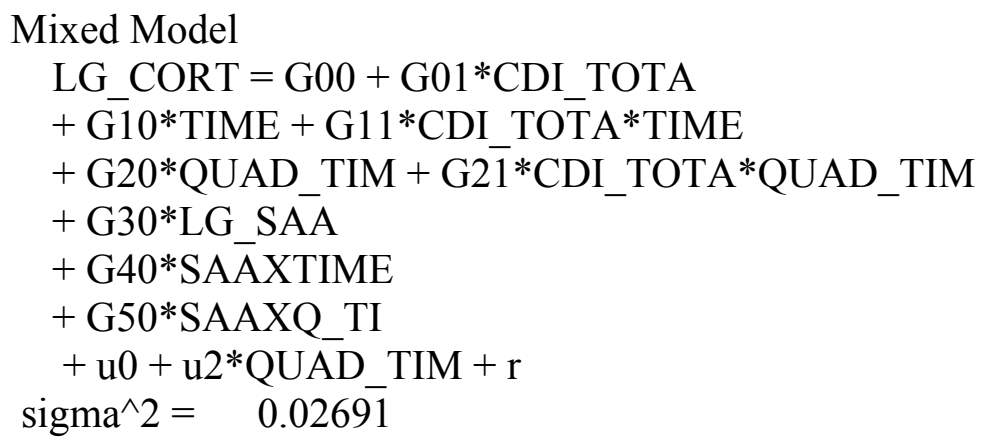

Standard errors of tau

$\begin{array}{lcc}\text { INTRCPT1,B0 } & 0.01366 & 0.00254 \\ \text { QUAD_TIM,B2 } & 0.00254 & 0.00084\end{array}$

tau (as correlations)

INTRCPT1,B0 $1.000-0.563$

QUAD_TIM,B2 -0.563 1.000

Random level-1 coefficient Reliability estimate

$\begin{array}{lc}\text { INTRCPT1, G0 } & 0.893 \\ \text { QUAD_TIM, G2 } & 0.653\end{array}$

The value of the log-likelihood function at iteration $12=1.015602 \mathrm{E}+001$

Final estimation of fixed effects:

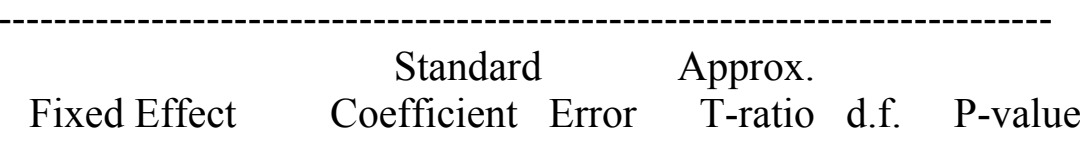




\begin{tabular}{|c|c|c|c|c|c|}
\hline \multicolumn{6}{|c|}{ For INTRCPT1, B0 } \\
\hline INTRCPT2, G00 & 0.575841 & 0.041021 & 14.038 & 34 & 0.000 \\
\hline CDI_TOTA, G01 & -0.005401 & 0.006587 & -0.820 & 34 & 0.418 \\
\hline \multicolumn{6}{|c|}{ For TIME slope, B1 } \\
\hline INTRCPT2, G10 & 0.019895 & 0.011801 & 1.686 & 101 & 0.095 \\
\hline CDI_TOTA, G11 & -0.000805 & 0.001831 & -0.440 & 101 & 0.661 \\
\hline \multicolumn{6}{|c|}{ For QŪAD_TIM slope, B2 } \\
\hline INTRCPT2, G20 & -0.014358 & 0.011069 & -1.297 & 34 & 0.203 \\
\hline CDI_TOTA, G21 & 0.003700 & 0.001763 & 2.098 & 34 & 0.043 \\
\hline \multicolumn{6}{|c|}{ For LG_SAA slope, B3 } \\
\hline INTRCPT2, G30 & 0.006402 & 0.130299 & 0.049 & 101 & 0.961 \\
\hline \multicolumn{6}{|c|}{ For SAAXTIME slope, B4 } \\
\hline INTRCPT2, G40 & -0.038118 & 0.097847 & -0.390 & 101 & 0.698 \\
\hline \multicolumn{6}{|c|}{ For SAAXQ_TI slope, B5 } \\
\hline INTRCPT2, G50 & 0.063729 & 0.058371 & 1.092 & 101 & 0.278 \\
\hline
\end{tabular}

Final estimation of variance components:

Random Effect Standard Variance df Chi-square P-value Deviation Component

$\begin{array}{lllllll}\text { INTRCPT1, u0 } & 0.22774 & 0.05187 & 34 & 354.24107 & 0.000\end{array}$ QUAD_TIM slope, u2 $\quad 0.04804 \quad 0.00231 \quad 34 \quad 106.28432 \quad 0.000$ $\begin{array}{llll}\text { level-1, } & \mathrm{r} & 0.16404 & 0.02691\end{array}$

Statistics for the current model

Deviance $\quad=-20.312047$

Number of estimated parameters $=13$

Model comparison test

$\begin{array}{ll}\text { Chi-square statistic } & =6.10473 \\ \text { Degrees of freedom } & =2 \\ \text { P-value }= & 0.046\end{array}$

\section{Model 12}

Level-1 Model 


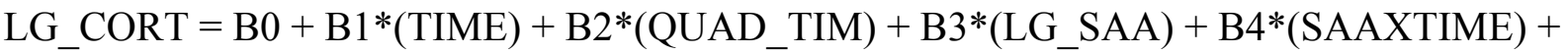
$\mathrm{B} 5 *\left(\mathrm{SAAX} \mathrm{Q}_{-} \mathrm{TI}\right)+\mathrm{r}$

Level-2 Model

$$
\begin{aligned}
& \mathrm{B} 0=\mathrm{G} 00+\mathrm{G} 01 *(\mathrm{CDI} \text { TOTA })+\mathrm{u} 0 \\
& \mathrm{~B} 1=\mathrm{G} 10+\mathrm{G} 11 *(\mathrm{CDI} \text { ITOTA }) \\
& \mathrm{B} 2=\mathrm{G} 20+\mathrm{G} 21 *(\mathrm{CDI} \text { ITOTA })+\mathrm{u} 2 \\
& \mathrm{~B} 3=\mathrm{G} 30+\mathrm{G} 31 *(\mathrm{CDI} \text { _TOTA }) \\
& \mathrm{B} 4=\mathrm{G} 40 \\
& \mathrm{~B} 5=\mathrm{G} 50
\end{aligned}
$$

LG_SAA has been centered around the group mean.

CDI_TOTA has been centered around the grand mean.

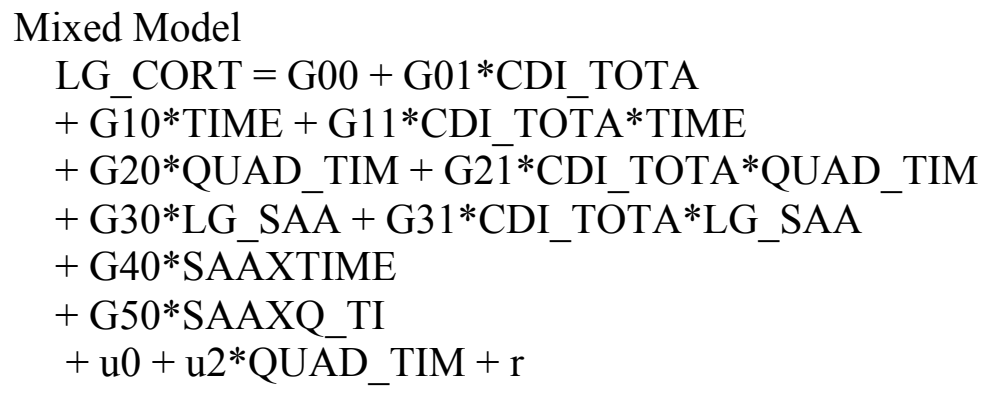

Random level-1 coefficient Reliability estimate

$\begin{array}{lc}\text { INTRCPT1, G0 } & 0.893 \\ \text { QUAD_TIM, G2 } & 0.654\end{array}$


The value of the log-likelihood function at iteration $12=1.027566 \mathrm{E}+001$

The outcome variable is LG_CORT

Final estimation of fixed effects:

\begin{tabular}{|c|c|c|c|c|c|}
\hline Fixed Effect & $\begin{array}{c}\text { Standard } \\
\text { Coefficient Erro }\end{array}$ & $\begin{array}{r}\text { Approx. } \\
\text { or } \quad \text { T-ratio }\end{array}$ & d.f. & P-value & \\
\hline \multicolumn{6}{|c|}{ For INTRCPT1, B0 } \\
\hline INTRCPT2, G00 & 0.576236 & 0.041049 & 14.038 & 34 & 0.000 \\
\hline CDI_TOTA, G01 & -0.005265 & 0.006596 & -0.798 & 34 & 0.430 \\
\hline \multicolumn{6}{|c|}{ For TIME slope, B1 } \\
\hline INTRCPT2, G10 & 0.019482 & 0.011809 & 1.650 & 100 & 0.102 \\
\hline CDI_TOTA, G11 & -0.001036 & 0.001887 & -0.549 & 100 & 0.584 \\
\hline \multicolumn{6}{|c|}{ For QŪAD TIM slope, B2 } \\
\hline INTRCPT̄2, G20 & -0.014466 & 0.011 & -1.307 & 34 & 0.200 \\
\hline CDI TOTA, G21 & 0.003549 & 0.00 & 1.984 & 34 & 0.055 \\
\hline \multicolumn{6}{|c|}{ For LG_SAA slope, B3 } \\
\hline INTRCPT2, G30 & 0.002433 & 0.130482 & 0.019 & 100 & 0.985 \\
\hline CDI_TOTA, G31 & -0.009091 & 0.018528 & -0.491 & 100 & 0.625 \\
\hline \multicolumn{6}{|c|}{ For SAAXTIME slope, B4 } \\
\hline INTRCPT2, G40 & -0.039123 & 0.097700 & -0.400 & 100 & 0.690 \\
\hline \multicolumn{6}{|c|}{ For SAAXQ_TI slope, B5 } \\
\hline INTRCPT2, G50 & 0.062574 & 0.058387 & 1.072 & 100 & 0.286 \\
\hline
\end{tabular}

Final estimation of variance components:

Random Effect Standard Variance df Chi-square P-value Deviation Component

$\begin{array}{lllllll}\text { INTRCPT1， u0 } & 0.22794 & 0.05196 & 34 & 356.00490 & 0.000\end{array}$

QUAD_TIM slope, u2 $\quad 0.04810 \quad 0.00231 \quad 34 \quad 106.68700 \quad 0.000$ $\begin{array}{llll}\text { level-1, } & \mathrm{r} & 0.16373 & 0.02681\end{array}$

Statistics for the current model

Deviance $\quad=-20.551316$

Number of estimated parameters $=14$

Model comparison test

Chi-square statistic $=0.23927$ 
Degrees of freedom $=1$

P-value $\quad=\quad>.500$

\section{Model 13}

Level-1 Model

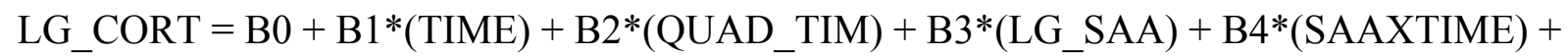
$\mathrm{B} 5 *(\mathrm{SAAX} \overline{\mathrm{X}} \mathrm{TI})+\mathrm{r}$

Level-2 Model

$$
\begin{aligned}
& \mathrm{B} 0=\mathrm{G} 00+\mathrm{G} 01 *(\mathrm{CDI} \text { TOTA })+\mathrm{u} 0 \\
& \mathrm{~B} 1=\mathrm{G} 10+\mathrm{G} 11 *(\text { CDI_TOTA }) \\
& \mathrm{B} 2=\mathrm{G} 20+\mathrm{G} 21 *(\text { CDI_TOTA })+\mathrm{u} 2 \\
& \mathrm{~B} 3=\mathrm{G} 30+\mathrm{G} 31 *(\text { CDI_TOTA }) \\
& \mathrm{B} 4=\mathrm{G} 40+\mathrm{G} 41 *(\text { CDI_TOTA }) \\
& \mathrm{B} 5=\mathrm{G} 50+\mathrm{G} 51 *(\text { CDI_TOTA })
\end{aligned}
$$

LG_SAA has been centered around the group mean.

CDI_TOTA has been centered around the grand mean.

$$
\begin{aligned}
& \text { Mixed Model } \\
& \text { LG_CORT }=\text { G00 + G01*CDI_TOTA } \\
& \text { + G10*TIME + G11*CDI_TOTA*TIME } \\
& \text { + G20*QUAD_TIM + G2 } 1 * \text { CDI_TOTA*QUUAD_TIM } \\
& \text { + G30*LG_SAA + G31*CDI_TOTA*LG_SAA } \\
& \text { + G40*SAAXTIME + G4 } 1{ }^{*} \text { CDI_TOTA*SAAXTIME } \\
& \text { + G50*SAAXQ_TI + G5 } 1 * \text { CDI_TOTA*SAAXQ_TI } \\
& \text { + u0 + u2*QUAD_TIM + r }
\end{aligned}
$$

$\operatorname{sigma}^{\wedge} 2=0.02632$

Standard error of $\operatorname{sigma}^{\wedge} 2=0.00361$

tau

$$
\begin{array}{llc}
\text { INTRCPT1,B0 } & 0.05150 & -0.00615 \\
\text { QUAD_TIM,B2 } & -0.00615 & 0.00237
\end{array}
$$

Standard errors of tau

$$
\begin{array}{lcc}
\text { INTRCPT1,B0 } & 0.01354 & 0.00254 \\
\text { QUAD_TIM,B2 } & 0.00254 & 0.00085
\end{array}
$$

tau (as correlations) 
INTRCPT1,B0 $1.000-0.556$

QUAD_TIM,B2 -0.556 1.000

Random level-1 coefficient Reliability estimate

$\begin{array}{lc} & \\ \text { INTRCPT1, G0 } & 0.894 \\ \text { QUAD_TIM, G2 } & 0.664\end{array}$

The value of the log-likelihood function at iteration $12=1.127346 \mathrm{E}+001$

The outcome variable is LG_CORT

Final estimation of fixed effects:

\begin{tabular}{|c|c|c|c|c|c|}
\hline Fixed Effect & $\begin{array}{c}\text { Standard } \\
\text { Coefficient Erro }\end{array}$ & $\begin{array}{r}\text { Approx. } \\
\text { or } \quad \text { T-ratio }\end{array}$ & d.f. & $\mathrm{P}$-value & \\
\hline \multicolumn{6}{|c|}{ For INTRCPT1, B0 } \\
\hline INTRCPT2, G00 & 0.576468 & 0.040849 & 14.112 & 34 & 0.000 \\
\hline CDI_TOTA, G01 & 4632 & 0.00 & & & 0.486 \\
\hline \multicolumn{6}{|c|}{ For TIME slope, B1 } \\
\hline INTRCPT2, G10 & 018425 & 0.011 & 1.570 & 98 & 0.120 \\
\hline CDI_TOTA, G11 & 91 & 0.00 & & 98 & 0.434 \\
\hline \multicolumn{6}{|c|}{ For QŪAD_TIM slope, B2 } \\
\hline INTRCPT2, G20 & -0.015360 & 0.01 & -1. & 34 & 0.177 \\
\hline CDI_TOTA, G21 & & 0.0 & & & 0.086 \\
\hline \multicolumn{6}{|c|}{ For LG_SAA slope, B3 } \\
\hline INTRCPT2, G30 & 41 & 0.13 & 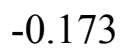 & 98 & 0.863 \\
\hline CDI_TOTA, G & 68 & 0.0 & & 8 & 0.565 \\
\hline \multicolumn{6}{|c|}{ For SAAXTIME slope, B4 } \\
\hline INTRCPT2, G40 & -0.076497 & 0.101 & -0.7 & 98 & 0.453 \\
\hline CDI_TOTA, G41 & -0.029327 & 0.022228 & -1.319 & 98 & 0.190 \\
\hline \multicolumn{6}{|c|}{ For SĀAXQ_TI slope, B5 } \\
\hline INTRC & 0046 & & & 98 & 0.44 \\
\hline CDI_TOTA, G51 & -0.016100 & 0.012472 & -1.291 & 98 & 0.200 \\
\hline
\end{tabular}

Final estimation of variance components:

Random Effect Standard Variance df Chi-square P-value Deviation Component

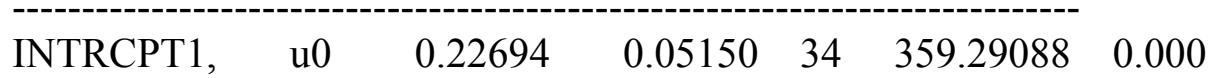

QUAD_TIM slope, u2 $\quad 0.04872 \quad 0.00237 \quad 34 \quad 109.98901 \quad 0.000$

$\begin{array}{llll}\text { level-1, } & \mathrm{r} & 0.16224 & 0.02632\end{array}$ 
Statistics for the current model

Deviance $\quad=-22.546928$

Number of estimated parameters $=16$

Model comparison test

Chi-square statistic $=1.99561$

Degrees of freedom $=2$

$\mathrm{P}$-value $\quad=\quad>.500$

\section{Model 14}

Level-1 Model

$$
\text { LG_CORT }=\mathrm{B} 0+\mathrm{B} 1 *(\mathrm{TIME})+\mathrm{B} 2 *\left(\mathrm{QUAD} \_\mathrm{TIM}\right)+\mathrm{r}
$$

Level-2 Model

$$
\begin{aligned}
& \mathrm{B} 0=\mathrm{G} 00+\mathrm{u} 0 \\
& \mathrm{~B} 1=\mathrm{G} 10 \\
& \mathrm{~B} 2=\mathrm{G} 20+\mathrm{u} 2
\end{aligned}
$$

Mixed Model

LG_CORT $=$ G00

+ G10*TIME

+ G20*QUAD_TIM $+\mathrm{u} 0+\mathrm{u} 2 *$ QUAD_TIM $+\mathrm{r}$

$\operatorname{sigma}^{\wedge} 2=0.03125$

Standard error of $\operatorname{sigma}^{\wedge} 2=\quad 0.00350$

tau

INTRCPT1,B0 $\quad 0.06080 \quad-0.00648$

QUAD_TIM,B2 -0.00648 0.00159

Standard errors of tau

INTRCPT1,B0 $0.01478 \quad 0.00227$

QUAD_TIM,B2 $0.00227 \quad 0.00056$

tau (as correlations)

INTRCPT1,B0 $1.000-0.658$ 
QUAD_TIM,B2 -0.658 1.000

Random level-1 coefficient Reliability estimate

$\begin{array}{lc}\text { INTRCPT1, G0 } & 0.921 \\ \text { QUAD_TIM, G2 } & 0.646\end{array}$

The value of the log-likelihood function at iteration $9=1.052137 \mathrm{E}+001$

The outcome variable is LG_CORT

Final estimation of fixed effects:

\begin{tabular}{|c|c|c|c|c|c|}
\hline Fixed Effect & $\begin{array}{c}\text { Standard } \\
\text { Coefficient Erro }\end{array}$ & $\begin{array}{r}\text { Approx. } \\
\text { T-ratio }\end{array}$ & d.f. & P-value & \\
\hline \multicolumn{6}{|c|}{ For INTRCPT1, B0 } \\
\hline \multicolumn{6}{|c|}{ For TIME slope, B1 } \\
\hline INTRCPT2, G10 & 0.007899 & 0.006683 & 1.182 & 158 & 0.23 \\
\hline \multicolumn{6}{|c|}{ For QUAD TIM slope, B2 } \\
\hline INTRCPT̄2, G20 & -0.018134 & 0.007856 & -2.308 & 39 & 0.02 \\
\hline
\end{tabular}

Final estimation of variance components:

Random Effect Standard Variance df Chi-square P-value Deviation Component

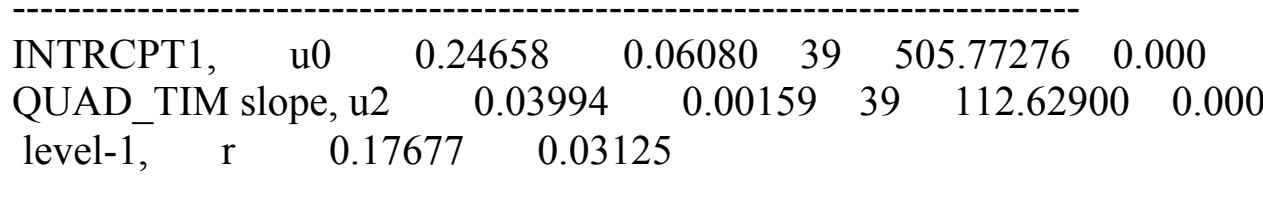

Statistics for the current model

Deviance $\quad=-21.042742$

Number of estimated parameters $=7$

Model comparison test

Chi-square statistic $=31.97181$

Degrees of freedom $=2$

$\mathrm{P}$-value $\quad=\quad<0.001$ 


\section{Model 15}

Level-1 Model

$$
\text { LG_CORT }=\mathrm{B} 0+\mathrm{B} 1 *(\mathrm{TIME})+\mathrm{B} 2 *\left(\mathrm{QUAD} \_\mathrm{TIM}\right)+\mathrm{r}
$$

Level-2 Model

$$
\begin{aligned}
& \mathrm{B} 0=\mathrm{G} 00+\mathrm{G} 01 *(\mathrm{CDI} \text { _TOTA })+\mathrm{u} 0 \\
& \mathrm{~B} 1=\mathrm{G} 10 \\
& \mathrm{~B} 2=\mathrm{G} 20+\mathrm{u} 2
\end{aligned}
$$

CDI_TOTA has been centered around the grand mean.

$$
\begin{aligned}
& \text { Mixed Model } \\
& \text { LG_CORT }=\mathrm{G} 00+\mathrm{G} 01 * \text { CDI_TOTA } \\
& + \text { G10*TIME } \\
& + \text { G20*QUAD_TIM } \\
& \quad+\mathrm{u} 0+\mathrm{u} 2 * \text { QUAD_TIM }+\mathrm{r}
\end{aligned}
$$

$\operatorname{sigma}^{\wedge} 2=0.03125$

Standard error of $\operatorname{sigma}^{\wedge} 2=\quad 0.00350$

$$
\begin{array}{lcc}
\text { tau } & & \\
\text { INTRCPT1,B0 } & 0.06015 & -0.00637 \\
\text { QUAD_TIM,B2 } & -0.00637 & 0.00160
\end{array}
$$

Standard errors of tau

$\begin{array}{lcc}\text { INTRCPT1,B0 } & 0.01463 & 0.00225 \\ \text { QUAD_TIM,B2 } & 0.00225 & 0.00056\end{array}$

tau (as correlations)

INTRCPT1,B0 $1.000-0.650$

QUAD_TIM,B2 -0.650 1.000

Random level-1 coefficient Reliability estimate

$\begin{array}{lc}\text { INTRCPT1, G0 } & 0.920 \\ \text { QUAD_TIM, G2 } & 0.646\end{array}$

The value of the log-likelihood function at iteration $17=1.053410 \mathrm{E}+001$ 
The outcome variable is LG_CORT

Final estimation of fixed effects:

\begin{tabular}{|c|c|c|c|c|c|}
\hline Fixed Effect & $\begin{array}{c}\text { Standard } \\
\text { Coefficient Erro }\end{array}$ & 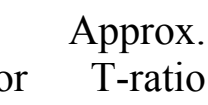 & d.f. & P-value & \\
\hline \multicolumn{6}{|c|}{ For INTRCPT1, B0 } \\
\hline INTRCPT2, G0C & 0.570171 & 0.041651 & 13.689 & 38 & 0.000 \\
\hline CDI TOTA, G0 & -0.000996 & 0.005736 & -0.174 & 38 & 0.863 \\
\hline \multicolumn{6}{|c|}{ For TIME slope, B1 } \\
\hline INTRCPT2, G10 & 0.007898 & 0.006683 & 1.182 & 158 & 0.239 \\
\hline \multicolumn{6}{|c|}{ For QUAD_TIM slope, B2 } \\
\hline INTRCPT̄2, G2C & -0.018132 & 0.007856 & -2.308 & 39 & 0.026 \\
\hline
\end{tabular}

Final estimation of variance components:

Random Effect Standard Variance df Chi-square P-value Deviation Component

$\begin{array}{lllllll} & & & & \\ \text { INTRCPT1, u0 } & 0.24525 & 0.06015 & 38 & 500.72287 & 0.000 \\ \text { QUAD_TIM slope, u2 } & 0.03994 & 0.00160 & 39 & 112.63293 & 0.000 \\ \text { level-1, } \quad \text { r } & 0.17677 & 0.03125 & & & & \end{array}$

Statistics for the current model

Deviance $\quad=-21.068192$

Number of estimated parameters $=8$

Model comparison test

Chi-square statistic $=0.02545$

Degrees of freedom $=1$

$\mathrm{P}$-value $\quad=\quad>.500$

\section{Model 16}

Level-1 Model

$$
\text { LG_CORT }=\text { B0 + B1* (TIME })+ \text { B2* }(\text { QUAD_TIM })+r
$$

Level-2 Model 


$$
\begin{aligned}
& \mathrm{B} 0=\mathrm{G} 00+\mathrm{G} 01 *(\mathrm{CDI} \text { TOTA })+\mathrm{u} 0 \\
& \mathrm{~B} 1=\mathrm{G} 10+\mathrm{G} 11 *(\mathrm{CDI} \text { ITOTA }) \\
& \mathrm{B} 2=\mathrm{G} 20+\mathrm{G} 21 *(\mathrm{CDI} \text { _TOTA })+\mathrm{u} 2
\end{aligned}
$$

CDI_TOTA has been centered around the grand mean.

$$
\begin{aligned}
& \text { Mixed Model } \\
& \text { LG_CORT }=\mathrm{G} 00+\mathrm{G} 01 * \text { CDI_TOTA } \\
& + \text { G10*TIME }+ \text { G } 11 * \text { CDI_TOTA*TIME } \\
& + \text { G20*QUAD_TIM }+ \text { G } 21 * \text { CDI_TOTA*QUAD_TIM } \\
& +\mathrm{u} 0+\mathrm{u} 2 * \text { QUAD_TIM }+\mathrm{r}
\end{aligned}
$$

$\operatorname{sigma}^{\wedge} 2=0.03001$

Standard error of $\operatorname{sigma}^{\wedge} 2=0.00337$

tau

INTRCPT1,B0 $0.05778 \quad-0.00538$

QUAD_TIM,B2 -0.00538 0.00125

Standard errors of tau

$\begin{array}{lll}\text { INTRCPT1,B0 } & 0.01406 & 0.00200\end{array}$

QUAD_TIM,B2 $0.00200 \quad 0.00048$

tau (as correlations)

\begin{tabular}{|c|c|c|}
\hline Fixed Effect & $\begin{array}{c}\text { Standard } \\
\text { Coefficient Error }\end{array}$ & $\begin{array}{l}\text { Approx. } \\
\text { T-ratio d.f. }\end{array}$ \\
\hline
\end{tabular}

INTRCPT1,B0 $1.000-0.632$

QUAD_TIM,B2 -0.632 1.000

Random level-1 coefficient Reliability estimate

$\begin{array}{lc}\text { INTRCPT1, G0 } & 0.920 \\ \text { QUAD_TIM, G2 } & 0.599\end{array}$

The value of the log-likelihood function at iteration $9=1.704713 \mathrm{E}+001$

The outcome variable is LG_CORT

Final estimation of fixed effects:

For INTRCPT1, B0 


$\begin{array}{lccccc}\text { INTRCPT2, G00 } & 0.570134 & 0.040821 & 13.967 & 38 & 0.000 \\ \text { CDI_TOTA, G01 } & -0.005225 & 0.006706 & -0.779 & 38 & 0.441 \\ \text { For_TIME slope, B1 } & & & & & \\ \text { INTRCPT2, G10 } & 0.007889 & 0.006550 & 1.205 & 157 & 0.230 \\ \text { CDI_TOTA, G11 } & -0.002731 & 0.001076 & -2.539 & 157 & 0.012 \\ \text { For QUAD_TIM slope, B2 } & & & & & \\ \text { INTRCPT2, G20 } & -0.018110 & 0.007232 & -2.504 & 38 & 0.017 \\ \text { CDI_TOTA, G21 } & 0.003207 & 0.001187 & 2.701 & 38 & 0.010\end{array}$

Final estimation of variance components:

Random Effect Standard Variance df Chi-square P-value Deviation Component

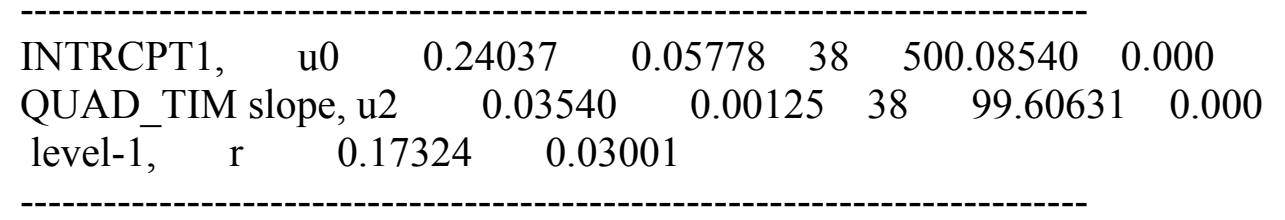

Statistics for the current model

Deviance $=-34.094262$

Number of estimated parameters $=10$

Model comparison test

Chi-square statistic $=13.02607$

Degrees of freedom $=2$

P-value $\quad=0.002$

\section{Model 17}

Level-1 Model

$$
\text { LG_CORT }=\text { B0 + B1* (TIME })+ \text { B2* }(\text { QUAD_TIM })+r
$$

Level-2 Model

$$
\begin{aligned}
& \text { B0 }=\text { G00 }+ \text { G0 } 1 *(\text { CHILD_EA })+\text { G02* }(\text { CHILD_WA })+\text { G03*(CDI_TOTA })+ \\
& \text { G04*(CHILD_SE }) \\
& + \text { G05*(CHILD_AG })+ \text { G06*(ETHNICIT })+\mathrm{u} 0 \\
& \text { B } 1=\text { G10 + G1 } 1 *(\text { CDI_TOTA }) \\
& \text { B2 }=\text { G } 20+\text { G } 21 *(\text { CDI_TOTA })+\mathrm{u} 2
\end{aligned}
$$


CHILD_EA CHILD_WA CDI_TOTA CHILD_SE CHILD_AG ETHNICIT have been centered around the grand mean.

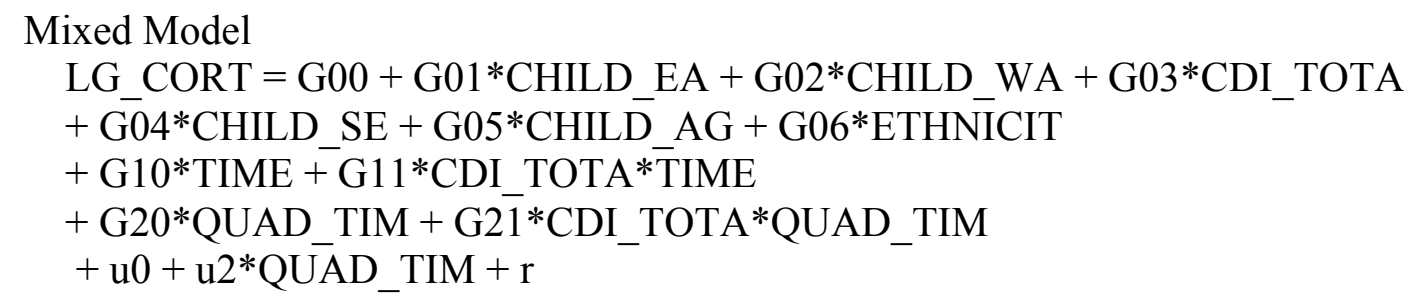

$\operatorname{sigma}^{\wedge} 2=0.03001$

Standard error of $\operatorname{sigma}^{\wedge} 2=\quad 0.00337$

tau

$\begin{array}{llr}\text { INTRCPT1,B0 } & 0.05290 & -0.00498 \\ \text { QUAD TIM,B2 } & -0.00498 & 0.00125\end{array}$

Standard errors of tau

INTRCPT1,B0 $0.01297 \quad 0.00191$

QUAD_TIM,B2 $0.00191 \quad 0.00048$

tau (as correlations)

INTRCPT1,B0 $1.000-0.611$

QUAD_TIM,B2 -0.611 1.000

Random level-1 coefficient Reliability estimate

$\begin{array}{lc}\text { INTRCPT1, G0 } & 0.913 \\ \text { QUAD_TIM, G2 } & 0.599\end{array}$

The value of the log-likelihood function at iteration $17=1.825936 \mathrm{E}+001$

Final estimation of fixed effects:

\begin{tabular}{|c|c|c|c|c|c|}
\hline Fixed Effect & $\begin{array}{c}\text { Standard } \\
\text { Coefficient Err }\end{array}$ & $\begin{array}{r}\text { Approx } \\
\text { T-rati }\end{array}$ & d.f. & \multicolumn{2}{|l|}{ P-value } \\
\hline \multicolumn{6}{|c|}{ For INTRCPT1, B0 } \\
\hline INTRCPT2, G00 & 0.570118 & 0.039298 & 14.507 & 33 & 0.000 \\
\hline CHILD_EA, G01 & 0.000008 & 0.000009 & 0.835 & 33 & 0.410 \\
\hline CHILD_WA, G02 & 0.000001 & 0.000009 & 0.100 & 33 & 0.921 \\
\hline CDI_TŌTA, G03 & -0.004972 & 0.006692 & -0.743 & 33 & 0.463 \\
\hline CHIL̄D_SE, G04 & 0.052399 & 0.070454 & 0.744 & 33 & 0.462 \\
\hline
\end{tabular}




$\begin{array}{lccccc}\text { CHILD_AG, G05 } & -0.029413 & 0.053625 & -0.548 & 33 & 0.587 \\ \text { ETHNICIT, G06 } & -0.007570 & 0.012766 & -0.593 & 33 & 0.557 \\ \text { For TIME slope, B1 } & & & & & \\ \text { INTRCPT2, G10 } & 0.007885 & 0.006549 & 1.204 & 157 & 0.230 \\ \text { CDI_TOTA, G11 } & -0.002732 & 0.001076 & -2.539 & 157 & 0.012 \\ \text { For QUAD_TIM slope, B2 } & & & & \\ \text { INTRCPT2, G20 } & -0.018101 & 0.007234 & -2.502 & 38 & 0.017 \\ \text { CDI_TOTA, G21 } & 0.003208 & 0.001188 & 2.701 & 38 & 0.010\end{array}$

Final estimation of variance components:

Random Effect Standard Variance df Chi-square P-value Deviation Component

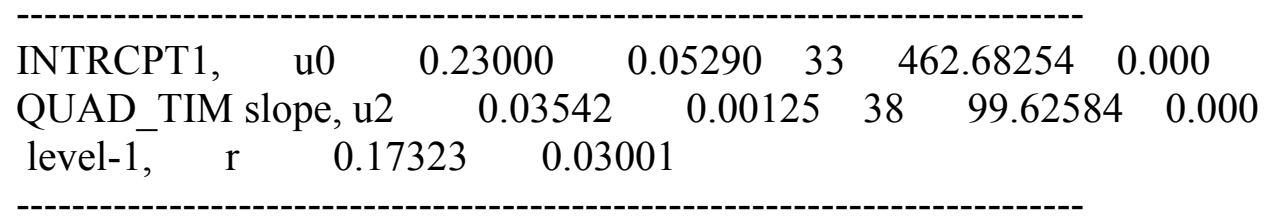

Statistics for the current model

Deviance $\quad=-36.518725$

Number of estimated parameters $=15$

Model comparison test

$\begin{array}{ll}\text { Chi-square statistic } & =2.42446 \\ \text { Degrees of freedom } & =5 \\ \text { P-value }= & >.500\end{array}$

\section{Model 18 - Final Model}

Level-1 Model

$$
\text { LG_CORT }=\text { B0 + B1* (TIME })+ \text { B2 } *\left(Q U A D \_T I M\right)+r
$$

Level-2 Model

$$
\begin{aligned}
& \mathrm{B} 0=\mathrm{G} 00+\mathrm{G} 01 *(\mathrm{CDI} \text { TOTA })+\mathrm{u} 0 \\
& \mathrm{~B} 1=\mathrm{G} 10+\mathrm{G} 11^{*}(\text { CDI_TOTA }) \\
& \mathrm{B} 2=\mathrm{G} 20+\mathrm{G} 21 *(\text { CDI_TOTA })+\mathrm{u} 2
\end{aligned}
$$

CDI_TOTA has been centered around the grand mean. 


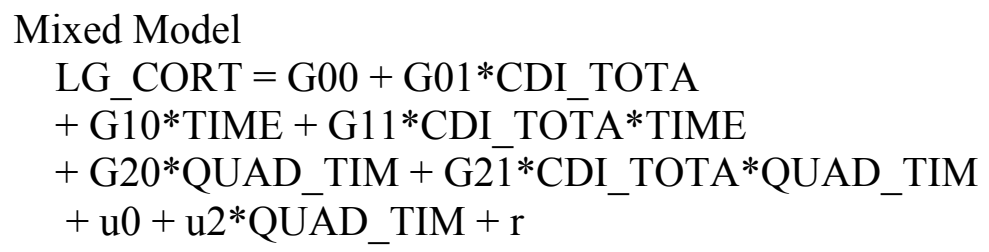

$\operatorname{sigma}^{\wedge} 2=0.03039$

tau

INTRCPT1,B0 $0.06101 \quad-0.00567$

QUAD_TIM,B2 -0.00567 0.00135

tau (as correlations)

INTRCPT1,B0 $1.000-0.623$

QUAD_TIM,B2 -0.623 1.000

Random level-1 coefficient Reliability estimate

$\begin{array}{lc}\text { INTRCPT1, G0 } & 0.923 \\ \text { QUAD_TIM, G2 } & 0.614\end{array}$

The value of the log-likelihood function at iteration $11=-9.425943 \mathrm{E}+000$

The outcome variable is LG_CORT

Final estimation of fixed effects:

\begin{tabular}{|c|c|c|c|c|c|}
\hline Fixed Effect & $\begin{array}{c}\text { Standard } \\
\text { Coefficient Erro }\end{array}$ & $\begin{array}{r}\text { Approx } \\
\text { or } \quad \text { T-ratic }\end{array}$ & d.f. & P-value & \\
\hline \multicolumn{6}{|c|}{ For INTRCPT1, B0 } \\
\hline INTRCPT2, G00 & 0.570116 & 0.041833 & 13.628 & 38 & 0.000 \\
\hline CDI_TOTA, G01 & -0.005227 & 0.006872 & -0.761 & 38 & 0.452 \\
\hline \multicolumn{6}{|c|}{ For TIME slope, B1 } \\
\hline INTRCPT2, G10 & 0.007885 & 0.006591 & 1.196 & 157 & 0.233 \\
\hline CDI_TOTA, G11 & -0.002732 & 0.001083 & -2.523 & 157 & 0.013 \\
\hline \multicolumn{6}{|c|}{ For QUAD_TIM slope, B2 } \\
\hline INTRCPT'2, G20 & -0.018100 & 0.007422 & -2.439 & 38 & 0.020 \\
\hline CDI_TOTA, G21 & 0.003208 & 0.001219 & 2.633 & 38 & 0.012 \\
\hline
\end{tabular}

The outcome variable is LG_CORT 
Final estimation of variance components:

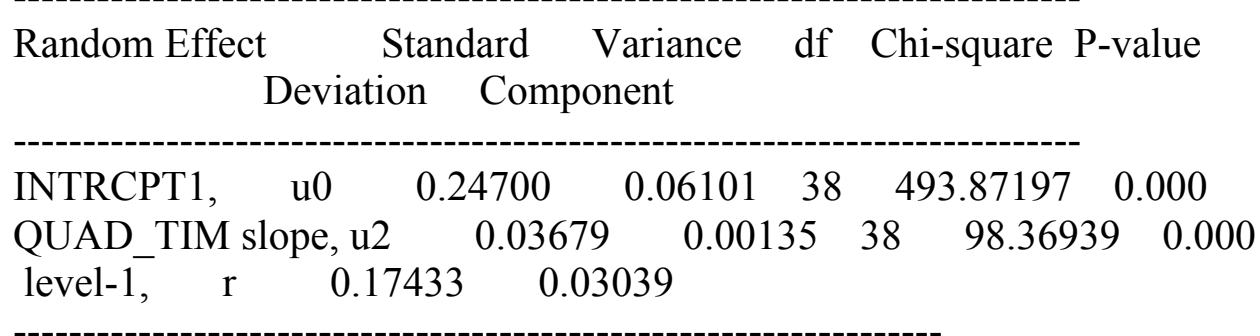

Statistics for current covariance components model

Deviance $\quad=18.851885$

Number of estimated parameters $=4$

Variance-Covariance components test

Chi-square statistic $=52.94615$

Degrees of freedom $=6$

$\mathrm{P}$-value $\quad=\quad<0.001$ 


\section{Appendix 4.2}

\section{Model Equations and Results: Maternal-Reported Internalizing in the TSST}

\section{Model 1:}

Level-1 Model

$$
\text { LG_CORT }=\mathrm{B} 0+\mathrm{r}
$$

Level-2 Model

$$
\mathrm{B} 0=\mathrm{G} 00+\mathrm{u} 0
$$

Mixed Model

LG_CORT $=\mathrm{G} 00+\mathrm{u} 0+\mathrm{r}$

$\operatorname{sigma}^{\wedge} 2=0.04527$

Standard error of $\operatorname{sigma}^{\wedge} 2=\quad 0.00454$

tau

INTRCPT1,B0 0.05815

Standard error of tau

INTRCPT1,B0 0.01472

Random level-1 coefficient Reliability estimate

INTRCPT1, G0 $\quad 0.885$

The value of the log-likelihood function at iteration $4=-1.154789 \mathrm{E}+001$

The outcome variable is LG_CORT

Final estimation of fixed effects:

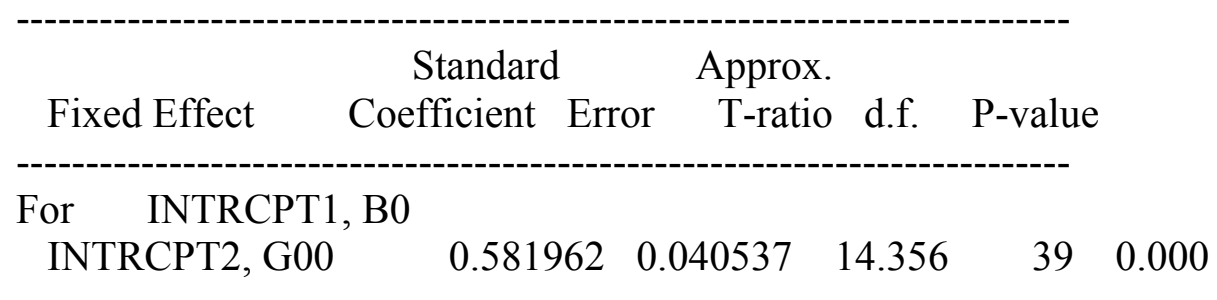


The outcome variable is LG_CORT

Final estimation of variance components:

\begin{tabular}{|c|c|c|c|c|}
\hline Random Effect & Standard & Variance & df & Chi-square \\
\hline & Deviation Com & onent & & \\
\hline $\begin{array}{l}\text { INTRCPT1, } \\
\text { level-1 }\end{array}$ & 0.24114 & 0.05815 & 39 & 347.85694 \\
\hline
\end{tabular}

Statistics for the current model

Deviance $\quad=23.095770$

Number of estimated parameters $=3$

\section{Model 2}

Level-1 Model

$$
\text { LG_CORT }=\mathrm{B} 0+\mathrm{B} 1 *(\mathrm{TIME})+\mathrm{r}
$$

Level-2 Model

$$
\begin{aligned}
& \mathrm{B} 0=\mathrm{G} 00+\mathrm{u} 0 \\
& \mathrm{~B} 1=\mathrm{G} 10
\end{aligned}
$$

Mixed Model

LG CORT $=\mathrm{G} 00$

$+\overline{\mathrm{G}} 10 * \mathrm{TIME}+\mathrm{u} 0+\mathrm{r}$

$\operatorname{sigma}^{\wedge} 2=0.04505$

Standard error of $\operatorname{sigma}^{\wedge} 2=0.00452$

tau

INTRCPT1,B0 0.05819

Standard error of tau

INTRCPT1,B0 0.01472 
Random level-1 coefficient Reliability estimate

INTRCPT1, G0 $\quad 0.885$

The value of the log-likelihood function at iteration $4=-1.105267 \mathrm{E}+001$

The outcome variable is LG_CORT

Final estimation of fixed effects:

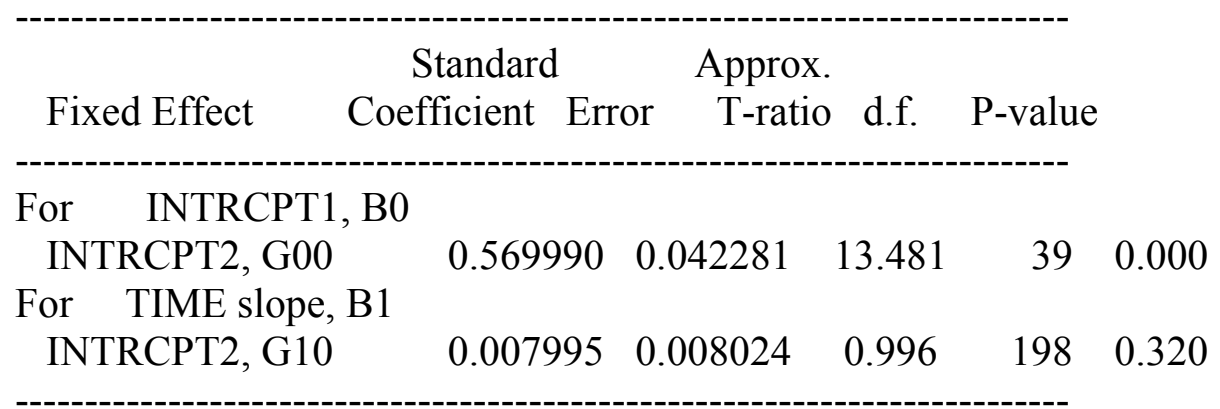

The outcome variable is LG_CORT

Final estimation of variance components:

\begin{tabular}{|c|c|c|c|c|c|}
\hline \multirow{2}{*}{\multicolumn{2}{|c|}{$\begin{array}{r}\text { Random Effect } \quad \text { Standard } \\
\text { Deviation Com }\end{array}$}} & Variance & $\mathrm{df}$ & Chi-square & \multirow[t]{2}{*}{$\mathrm{P}$-value } \\
\hline & & onent & & & \\
\hline \multirow{2}{*}{$\begin{array}{l}\text { INTRCPT1, } \\
\text { level-1, r }\end{array}$} & 0.24122 & 0.05819 & 39 & 349.61705 & 0.000 \\
\hline & 0.21224 & 4505 & & & \\
\hline
\end{tabular}

Statistics for the current model

Deviance $\quad=22.105337$

Number of estimated parameters $=4$

Model comparison test

Chi-square statistic $=0.99043$

Degrees of freedom $=1$

P-value $\quad=\quad>.500$

\section{Model 3}


Level-1 Model

$$
\mathrm{LG} \_\mathrm{CORT}=\mathrm{B} 0+\mathrm{B} 1 *(\mathrm{TIME})+\mathrm{B} 2 *\left(\mathrm{QUAD} \_\mathrm{TIM}\right)+\mathrm{r}
$$

Level-2 Model

$$
\begin{aligned}
& \mathrm{B} 0=\mathrm{G} 00+\mathrm{u} 0 \\
& \mathrm{~B} 1=\mathrm{G} 10 \\
& \mathrm{~B} 2=\mathrm{G} 20
\end{aligned}
$$

$$
\begin{aligned}
& \text { Mixed Model } \\
& \text { LG_CORT }=\text { G00 } \\
& + \text { G10*TIME } \\
& + \text { G20*QUAD_TIM }+\mathrm{u} 0+\mathrm{r}
\end{aligned}
$$

$\operatorname{sigma}^{\wedge} 2=0.04258$

Standard error of $\operatorname{sigma}^{\wedge} 2=\quad 0.00427$

tau

INTRCPT1,B0 0.05865

\begin{tabular}{|c|c|c|c|c|c|}
\hline Fixed Effect & $\begin{array}{c}\text { Standard } \\
\text { Coefficient Err }\end{array}$ & $\begin{array}{r}\text { Approx. } \\
\text { or } \quad \text { T-ratio }\end{array}$ & d.f. & P-value & \\
\hline \multicolumn{6}{|c|}{ For $\quad$ INTRCPT1, B0 } \\
\hline INTRCPT2, G00 & 0.570786 & 0.042204 & 13.525 & 39 & 0.000 \\
\hline \multicolumn{6}{|c|}{ For TIME slope, B1 } \\
\hline INTRCPT2, G10 & 0.008037 & 0.007801 & 1.030 & 197 & 0.304 \\
\hline \multicolumn{6}{|c|}{ For QUAD_TIM slope, B2 } \\
\hline INTRCPT2, G20 & -0.018488 & 0.005452 & -3.391 & 197 & 0.001 \\
\hline
\end{tabular}

Standard error of tau

INTRCPT1,B0 0.01473

Random level-1 coefficient Reliability estimate

INTRCPT1, G0 0.892

The value of the log-likelihood function at iteration $4=-5.464535 \mathrm{E}+000$

The outcome variable is LG_CORT

Final estimation of fixed effects: 
The outcome variable is LG_CORT

Final estimation of variance components:

Random Effect Standard Variance df Chi-square P-value Deviation Component

$\begin{array}{lllllll}\text { INTRCPT1, u0 } & 0.24219 & 0.05865 & 39 & 370.14487 & 0.000\end{array}$

$\begin{array}{llll}\text { level-1, } & \mathrm{r} & 0.20635 & 0.04258\end{array}$

Statistics for the current model

Deviance $\quad=10.929069$

Number of estimated parameters $=5$

Model comparison test

Chi-square statistic $=11.17627$

Degrees of freedom $=1$

P-value $\quad=0.001$

\section{Model 4}

Level-1 Model

$$
\text { LG_CORT }=\text { B0 + B1*(TIME })+ \text { B2 } *(\text { QUAD_TIM })+\text { B3 } *\left(L G \_S A A\right)+r
$$

Level-2 Model

$$
\begin{aligned}
& \mathrm{B} 0=\mathrm{G} 00+\mathrm{u} 0 \\
& \mathrm{~B} 1=\mathrm{G} 10 \\
& \mathrm{~B} 2=\mathrm{G} 20 \\
& \mathrm{~B} 3=\mathrm{G} 30
\end{aligned}
$$

LG_SAA has been centered around the group mean.

$$
\begin{aligned}
& \text { Mixed Model } \\
& \text { LG_CORT = G00 } \\
& + \text { G10*TIME } \\
& + \text { G20*QUAD_TIM } \\
& + \text { G30*LG_SAA } \\
& + \text { u0+ r }
\end{aligned}
$$


sigma $^{\wedge} 2=0.04378$

Standard error of $\operatorname{sigma}^{\wedge} 2=0.00520$

tau

INTRCPT1,B0 0.06191

Standard error of tau

INTRCPT1,B0 0.01672

Random level-1 coefficient Reliability estimate

INTRCPT1, G0 $\quad 0.875$

The value of the log-likelihood function at iteration $5=-1.152421 \mathrm{E}+001$

The outcome variable is LG_CORT

Final estimation of fixed effects:

\begin{tabular}{|c|c|c|c|c|c|}
\hline Fixed Effect & $\begin{array}{c}\text { Standard } \\
\text { Coefficient Erro }\end{array}$ & $\begin{array}{r}\text { Approx. } \\
\text { T-ratio }\end{array}$ & d.f. & P-value & \\
\hline \multicolumn{6}{|c|}{ For INTRCPT1, B0 } \\
\hline $\begin{array}{l}\text { INTRCPT2, G00 } \\
\text { For TIME slope. }\end{array}$ & $\begin{array}{l}0.574991 \\
\text { B1 }\end{array}$ & 0.045890 & 12.530 & 35 & 0.000 \\
\hline INTRCPT2, G10 & 0.021358 & 0.014592 & 1.464 & 139 & 0.146 \\
\hline \multicolumn{6}{|c|}{ For QUAD_TIM slope, B2 } \\
\hline INTRCPT2, G20 & -0.014418 & 0.009612 & -1.500 & 139 & 0.136 \\
\hline \multicolumn{6}{|c|}{ For LG_SAA slope, B3 } \\
\hline INTRCPT2, G30 & 0.253683 & 0.129530 & 1.958 & 139 & 0.052 \\
\hline
\end{tabular}

The outcome variable is LG_CORT

Final estimation of variance components:

Random Effect Standard Variance df Chi-square P-value Deviation Component

$\begin{array}{llllll}\text { INTRCPT1, u0 } & 0.24882 & 0.06191 & 35 & 289.72802 & 0.000\end{array}$
$\begin{array}{llll}\text { level-1, } & \text { r } & 0.20924 & 0.04378\end{array}$ 
Statistics for the current model

Deviance $\quad=23.048416$

Number of estimated parameters $=6$

Model comparison test

Chi-square statistic $=12.11935$

Degrees of freedom $=1$

$\mathrm{P}$-value $\quad=\quad<0.001$

\section{Model 5}

Level-1 Model

$$
\text { LG_CORT }=\mathrm{B} 0+\mathrm{B} 1 *(\mathrm{TIME})+\mathrm{B} 2 *\left(\mathrm{QUAD} \_\mathrm{TIM}\right)+\mathrm{B} 3 *\left(\mathrm{LG} \_\mathrm{SAA}\right)+\mathrm{B} 4 *(\mathrm{SAAXTIME})+\mathrm{r}
$$

Level-2 Model

$$
\begin{aligned}
& \mathrm{B} 0=\mathrm{G} 00+\mathrm{u} 0 \\
& \mathrm{~B} 1=\mathrm{G} 10 \\
& \mathrm{~B} 2=\mathrm{G} 20 \\
& \mathrm{~B} 3=\mathrm{G} 30 \\
& \mathrm{~B} 4=\mathrm{G} 40
\end{aligned}
$$

LG_SAA has been centered around the group mean.

$$
\begin{aligned}
& \text { Mixed Model } \\
& \text { LG_CORT = G00 } \\
& + \text { G10*TIME } \\
& + \text { G20*QUUA_TIM } \\
& + \text { G30*LG_SAA } \\
& + \text { G40*SAAXTIME } \\
& +\mathrm{u} 0+\mathrm{r} \\
& \text { sigma^2 }^{\wedge} \quad 0.04350
\end{aligned}
$$

Standard error of $\operatorname{sigma}^{\wedge} 2=0.00516$

tau

INTRCPT1,B0 0.06061 
Standard error of tau

INTRCPT1,B0 0.01640

Random level-1 coefficient Reliability estimate

INTRCPT1, G0 $\quad 0.873$

The value of the log-likelihood function at iteration $7=-1.071677 \mathrm{E}+001$

The outcome variable is LG_CORT

Final estimation of fixed effects:

\begin{tabular}{|c|c|c|c|c|c|}
\hline Fixed Effect & $\begin{array}{c}\text { Standard } \\
\text { Coefficient Err }\end{array}$ & $\begin{array}{r}\text { Approx } \\
\text { or } \quad \text { T-ratio }\end{array}$ & d.f. & P-value & \\
\hline \multicolumn{6}{|c|}{ For $\quad$ INTRCPT1, B0 } \\
\hline INTRCPT2, G00 & 0.574508 & 0.045468 & 12.636 & 35 & 0.000 \\
\hline \multicolumn{6}{|c|}{ For TIME slope, B1 } \\
\hline INTRCPT2, G10 & 0.024608 & 0.014766 & 1.666 & 138 & 0.098 \\
\hline \multicolumn{6}{|c|}{ For QUAD_TIM slope, B2 } \\
\hline INTRCPT2, G20 & -0.013325 & 0.009619 & -1.385 & 138 & 0.168 \\
\hline \multicolumn{6}{|c|}{ For LG_SAA slope, B3 } \\
\hline INTRCPT2, G30 & 0.175319 & 0.143004 & 1.226 & 138 & 0.222 \\
\hline \multicolumn{6}{|c|}{ For SAAXTIME slope, B4 } \\
\hline INTRCPT2, G40 & -0.102169 & 0.080155 & -1.275 & 138 & 0.205 \\
\hline
\end{tabular}

The outcome variable is $\mathrm{LG}_{-}$CORT

Final estimation of variance components:

Random Effect Standard Variance df Chi-square P-value Deviation Component

$\begin{array}{lllllll}\text { INTRCPT1, u0 } & 0.24618 & 0.06061 & 35 & 286.02241 & 0.000\end{array}$ $\begin{array}{llll}\text { level-1, } & \mathrm{r} & 0.20856 & 0.04350\end{array}$

Statistics for the current model

Deviance $\quad=21.433540$

Number of estimated parameters $=7$ 
Model comparison test

$\begin{array}{lll}\text { Chi-square statistic } & = & 1.61488 \\ \text { Degrees of freedom } & =1 \\ \text { P-value }= & 0.201\end{array}$

\section{Model 6}

Level-1 Model

LG_CORT $=$ B0 + B $1 *(T I M E)+B 2 *\left(Q U A D \_T I M\right)+B 3 *\left(L G \_S A A\right)+B 4 *(S A A X T I M E)+$ $\mathrm{B} 5 *\left(\mathrm{SAAX} \mathrm{X} \_\mathrm{TI}\right)+\mathrm{r}$

Level-2 Model

$$
\begin{aligned}
& \mathrm{B} 0=\mathrm{G} 00+\mathrm{u} 0 \\
& \mathrm{~B} 1=\mathrm{G} 10 \\
& \mathrm{~B} 2=\mathrm{G} 20 \\
& \mathrm{~B} 3=\mathrm{G} 30 \\
& \mathrm{~B} 4=\mathrm{G} 40 \\
& \mathrm{~B} 5=\mathrm{G} 50
\end{aligned}
$$

LG_SAA has been centered around the group mean.

$$
\begin{aligned}
& \text { Mixed Model } \\
& \text { LG_CORT }=\text { G00 } \\
& + \text { G10*TIME } \\
& + \text { G20*QUAD_TIM } \\
& + \text { G30*LG_SAA } \\
& + \text { G40*SAAXTIME } \\
& + \text { G50*SAAXQ_TI } \\
& + \text { + } 0+\text { r }
\end{aligned}
$$

$\operatorname{sigma}^{\wedge} 2=0.04339$

Standard error of $\operatorname{sigma}^{\wedge} 2=0.00515$

tau

INTRCPT1,B0 $\quad 0.06030$

Standard error of tau

INTRCPT1,B0 0.01632 
Random level-1 coefficient Reliability estimate

INTRCPT1, G0 $\quad 0.873$

The value of the log-likelihood function at iteration $7=-1.044688 \mathrm{E}+001$

The outcome variable is LG_CORT

Final estimation of fixed effects:

\begin{tabular}{|c|c|c|c|c|c|}
\hline Fixed Effect & $\begin{array}{c}\text { Standard } \\
\text { Coefficient Erro }\end{array}$ & $\begin{array}{r}\text { Approx. } \\
\text { or } \quad \text { T-ratio }\end{array}$ & d.f. & P-value & \\
\hline $\begin{array}{l}\text { For INTRCPT } \\
\text { INTRCPT2, G0 }\end{array}$ & $\begin{array}{ll}1, \mathrm{~B} 0 & \\
\mathrm{j}^{2} & 0.574178\end{array}$ & 0.045364 & 12.657 & 35 & 0.0 \\
\hline $\begin{array}{l}\text { For TIME slope } \\
\text { INTRCPT2, G1 }\end{array}$ & $b^{B} \quad 0.023018$ & ת & 12.00 & 137 & 0.125 \\
\hline For QUAD_TIM s & lope, B2 & 0009635 & 439 & 37 & 01 \\
\hline $\begin{array}{l}\text { For LG_SAA slo } \\
\text { INTRCPT2, G3 }\end{array}$ & pe, B3 0.190863 & 0.144380 & 1.322 & 137 & 0.188 \\
\hline $\begin{array}{l}\text { For SAAXTIME s } \\
\text { INTRCPT2, G4 }\end{array}$ & lope, B4 & 0.120208 & -0.302 & 137 & 0.763 \\
\hline $\begin{array}{l}\text { For SAAXQ_TI sl } \\
\text { INTRCPT2, G5C }\end{array}$ & $\begin{array}{l}\text { lope, B5 } \\
0.048619\end{array}$ & 0.066122 & 0.735 & 137 & 0.463 \\
\hline
\end{tabular}

The outcome variable is LG_CORT

Final estimation of variance components:

Random Effect Standard Variance df Chi-square P-value Deviation Component

\begin{tabular}{|c|c|c|c|c|}
\hline $\begin{array}{l}\text { INTRCPT1, } \\
\text { level-1, }\end{array}$ & $\begin{array}{lr}\text { u0 } & 0.24555 \\
0.20830 & 0 .\end{array}$ & $\begin{array}{l}0.06030 \\
4339\end{array}$ & 35 & 285.37308 \\
\hline
\end{tabular}

Statistics for the current model

Deviance $\quad=20.893763$

Number of estimated parameters $=8$

Model comparison test 
Chi-square statistic $=0.53978$

Degrees of freedom $=1$

$\mathrm{P}$-value $\quad=\quad>.500$

\section{Model 7}

Level-1 Model

LG_CORT $=$ B0 + B1 $*($ TIME $)+$ B2 $*\left(Q U A D \_T I M\right)+B 3 *\left(L G \_S A A\right)+B 4 *($ SAAXTIME $)+$ $\mathrm{B} 5 *(\mathrm{SAAX} \overline{\mathrm{Q}} \mathrm{TI})+\mathrm{r}$

Level-2 Model

$$
\begin{aligned}
& \mathrm{B} 0=\mathrm{G} 00+\mathrm{u} 0 \\
& \mathrm{~B} 1=\mathrm{G} 10+\mathrm{u} 1 \\
& \mathrm{~B} 2=\mathrm{G} 20+\mathrm{u} 2 \\
& \mathrm{~B} 3=\mathrm{G} 30 \\
& \mathrm{~B} 4=\mathrm{G} 40 \\
& \mathrm{~B} 5=\mathrm{G} 50
\end{aligned}
$$

LG_SAA has been centered around the group mean.

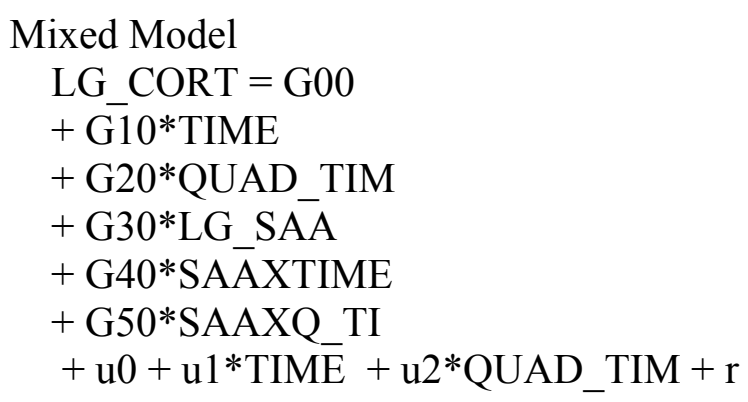

$\operatorname{sigma}^{\wedge} 2=0.02301$

Standard error of sigma^ $2=0.00387$

tau

INTRCPT1,B0 $0.05426 \quad-0.00050 \quad-0.00562$

TIME,B1 $-0.00050 \quad 0.00178 \quad-0.00136$

$\begin{array}{llll}\text { QUAD_TIM,B2 } & -0.00562 & -0.00136 & 0.00171\end{array}$

Standard errors of tau

$\begin{array}{llll}\text { INTRCPT1,B0 } & 0.01457 & 0.00322 & 0.00264\end{array}$

$\begin{array}{llll}\text { TIME,B1 } & 0.00322 & 0.00150 & 0.00079\end{array}$

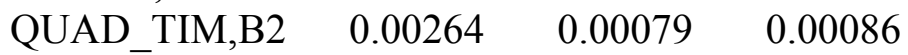


tau (as correlations)

INTRCPT1,B0 $1.000-0.051-0.583$

TIME,B1 -0.051 $1.000-0.780$

QUAD_TIM,B2 -0.583-0.780 1.000

Random level-1 coefficient Reliability estimate

$\begin{array}{lc}\text { INTRCPT1, G0 } & 0.876 \\ \text { TIME, G1 } & 0.310 \\ \text { QUAD_TIM, G2 } & 0.492\end{array}$

The outcome variable is LG_CORT

Final estimation of fixed effects:

\begin{tabular}{|c|c|c|c|c|c|}
\hline Fixed Effect & $\begin{array}{c}\text { Standard } \\
\text { Coefficient Erro }\end{array}$ & $\begin{array}{r}\text { Approx. } \\
\text { or } \quad \text { T-ratio }\end{array}$ & d.f. & P-value & \\
\hline \multicolumn{6}{|c|}{ For INTRCPT1, B0 } \\
\hline INTRCPT2, G00 & 0.578179 & 0.041389 & 13.969 & 35 & 0.000 \\
\hline \multicolumn{6}{|c|}{ For TIME slope, B1 } \\
\hline INTRCPT2, G10 & 0.018567 & 0.013039 & 1.424 & 35 & 0.163 \\
\hline \multicolumn{6}{|c|}{ For QUAD_TIM slope, B2 } \\
\hline INTRCPT'2, G20 & -0.015377 & 0.009896 & -1.554 & 35 & 0.129 \\
\hline \multicolumn{6}{|c|}{ For LG_SAA slope, B3 } \\
\hline INTRCPT2, G30 & 0.023501 & 0.135326 & 0.174 & 67 & 0.863 \\
\hline \multicolumn{6}{|c|}{ For SAAXTIME slope, B4 } \\
\hline INTRCPT2, G40 & -0.000986 & 0.096991 & -0.010 & 67 & 0.992 \\
\hline \multicolumn{6}{|c|}{ For SAAXQ_TI slope, B5 } \\
\hline INTRCPT2, G50 & 0.065483 & 0.054359 & 1.205 & 67 & 0.233 \\
\hline
\end{tabular}

The outcome variable is LG_CORT

Final estimation of variance components:

Random Effect Standard Variance df Chi-square P-value Deviation Component

\begin{tabular}{llllll}
\hline INTRCPT1, u0 & 0.23293 & 0.05426 & 35 & 308.83017 & 0.000
\end{tabular}

$\begin{array}{llllll}\text { TIME slope, u1 } \quad 0.04220 & 0.00178 & 35 & 46.71912 & 0.089\end{array}$

$\begin{array}{llllll}\text { QUAD_TIM slope, } \mathrm{u} 2 & 0.04137 & 0.00171 & 35 & 66.59813 & 0.001\end{array}$

level-1, $\quad r \quad 0.15168 \quad 0.02301$ 
Statistics for the current model

Deviance $\quad=-24.902481$

Number of estimated parameters $=13$

Model comparison test

Chi-square statistic $=45.79624$

Degrees of freedom $=5$

P-value $\quad=\quad<0.001$

\section{Model 8}

Level-1 Model

LG_CORT $=\mathrm{B} 0+\mathrm{B} 1 *(\mathrm{TIME})+\mathrm{B} 2 *\left(\mathrm{QUAD} \_\mathrm{TIM}\right)+\mathrm{B} 3 *(\mathrm{LG}$ _SAA $)+\mathrm{B} 4 *(\mathrm{SAAXTIME})+$ $\mathrm{B} 5 *(\mathrm{SAAXQ} \mathrm{TI})+\mathrm{r}$

Level-2 Model

$$
\begin{aligned}
& \mathrm{B} 0=\mathrm{G} 00+\mathrm{u} 0 \\
& \mathrm{~B} 1=\mathrm{G} 10 \\
& \mathrm{~B} 2=\mathrm{G} 20+\mathrm{u} 2 \\
& \mathrm{~B} 3=\mathrm{G} 30+\mathrm{u} 3 \\
& \mathrm{~B} 4=\mathrm{G} 40 \\
& \mathrm{~B} 5=\mathrm{G} 50
\end{aligned}
$$

LG_SAA has been centered around the group mean.

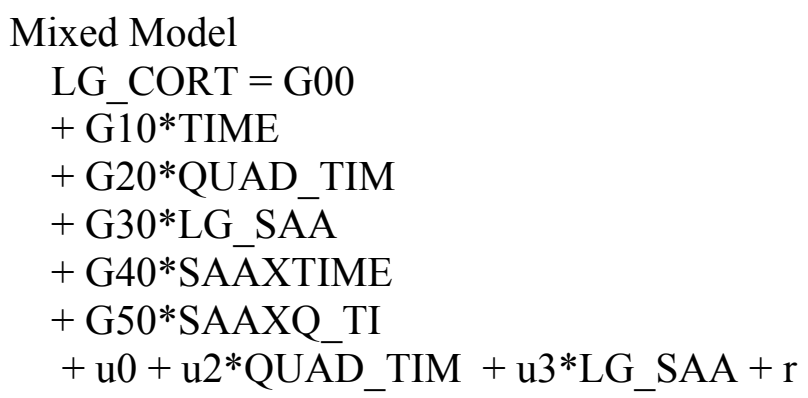

$\operatorname{sigma}^{\wedge} 2=0.02610$

Standard error of $\operatorname{sigma}^{\wedge} 2=0.00383$ 


$\begin{array}{llcc}\text { tau } & & & \\ \text { INTRCPT1,B0 } & 0.05567 & -0.00567 & 0.05148 \\ \text { QUAD_TIM,B2 } & -0.00567 & 0.00246 & 0.00106 \\ \text { LG_SAA,B3 } & 0.05148 & 0.00106 & 0.06877\end{array}$

Standard errors of tau

$\begin{array}{llll}\text { INTRCPT1,B0 } & 0.01452 & 0.00263 & 0.03051\end{array}$

$\begin{array}{llll}\text { QUAD_TIM,B2 } & 0.00263 & 0.00087 & 0.00684\end{array}$

LG_SAA,B3 $0.03051 \quad 0.00684 \quad 0.09499$

tau (as correlations)

INTRCPT1,B0 $1.000-0.4840 .832$

QUAD_TIM,B2 -0.484 $1.000 \quad 0.082$

LG_SAA,B3 0.8320 .0821 .000

Random level-1 coefficient Reliability estimate

$\begin{array}{lc}\text { INTRCPT1, G0 } & 0.897 \\ \text { QUAD_TIM, G2 } & 0.586 \\ \text { LG_SAA, G3 } & 0.110\end{array}$

The outcome variable is LG_CORT

Final estimation of fixed effects:

\begin{tabular}{|c|c|c|c|c|c|}
\hline Fixed Effect & Standard & Approx. & d.f. & P-value & \\
\hline \multicolumn{6}{|c|}{ For $\quad$ INTRCPT1, B0 } \\
\hline INTRCPT2, G00 & 0.579201 & 0.042204 & 13.724 & 35 & 0.000 \\
\hline \multicolumn{6}{|c|}{ For TIME slope, B1 } \\
\hline INTRCPT2, G10 & 0.018162 & 0.011711 & 1.551 & 67 & 0.126 \\
\hline \multicolumn{6}{|c|}{ For QUAD TIM slope, B2 } \\
\hline INTRCPT2, G20 & -0.014479 & 0.011209 & -1.292 & 35 & 0.205 \\
\hline \multicolumn{6}{|c|}{ For LG_SAA slope, B3 } \\
\hline INTRCPT2, G30 & 0.033248 & 0.138032 & 0.241 & 35 & 0.811 \\
\hline \multicolumn{6}{|c|}{ For SAAXTIME slope, B4 } \\
\hline INTRCPT2, G40 & -0.020239 & 0.096141 & -0.211 & 67 & 0.834 \\
\hline \multicolumn{6}{|c|}{ For SAAXQ_TI slope, B5 } \\
\hline INTRCPT2, G50 & 0.061312 & 0.058838 & 1.042 & 67 & 0.301 \\
\hline
\end{tabular}

The outcome variable is LG_CORT 
Final estimation of variance components:

Random Effect Standard Variance df Chi-square P-value Deviation Component

$\begin{array}{ccccccc}\text { INTRCPT1, u0 } & 0.23595 & 0.05567 & 35 & 343.33414 & 0.000 \\ \text { QUAD_TIM slope, u2 } & 0.04964 & 0.00246 & 35 & 84.54331 & 0.000 \\ \text { LG_SAA slope, u3 } & 0.26225 & 0.06877 & 35 & 26.12914 & >.500 \\ \text { level-1, r r } & 0.16154 & 0.02610 & & & \end{array}$

Statistics for the current model

Deviance $\quad=-17.717672$

Number of estimated parameters $=13$

Model comparison test

Chi-square statistic $=38.61144$

Degrees of freedom $=5$

$\mathrm{P}$-value $\quad=\quad<0.001$

\section{Model 9}

Level-1 Model

LG_CORT $=$ B0 + B $1 *($ TIME $)+$ B2 $*\left(Q U A D \_T I M\right)+B 3 *\left(L G \_S A A\right)+B 4 *(S A A X T I M E)+$ $\mathrm{B} 5 *(\mathrm{SAAXQ}$ TI $)+\mathrm{r}$

Level-2 Model

$$
\begin{aligned}
& \mathrm{B} 0=\mathrm{G} 00+\mathrm{u} 0 \\
& \mathrm{~B} 1=\mathrm{G} 10 \\
& \mathrm{~B} 2=\mathrm{G} 20+\mathrm{u} 2 \\
& \mathrm{~B} 3=\mathrm{G} 30 \\
& \mathrm{~B} 4=\mathrm{G} 40 \\
& \mathrm{~B} 5=\mathrm{G} 50
\end{aligned}
$$

LG_SAA has been centered around the group mean.

$$
\begin{aligned}
& \text { Mixed Model } \\
& \text { LG_CORT }=\text { G00 } \\
& + \text { G10*TIME } \\
& + \text { G20*QUAD_TIM }
\end{aligned}
$$




$$
\begin{aligned}
& + \text { G30*LG_SAA } \\
& + \text { G40*SAAXTIME } \\
& + \text { G50*SAAXQ_TI } \\
& +\mathrm{u} 0+\mathrm{u} 2 * \text { QUAD_TIM }+\mathrm{r}
\end{aligned}
$$

$$
\operatorname{sigma}^{\wedge} 2=0.02685
$$

Standard error of $\operatorname{sigma}^{\wedge} 2=0.00368$

tau

$$
\begin{array}{llc}
\text { INTRCPT1,B0 } & 0.05347 & -0.00709 \\
\text { QUAD_TIM,B2 } & -0.00709 & 0.00296
\end{array}
$$

Standard errors of tau

$\begin{array}{lll}\text { INTRCPT1,B0 } & 0.01403 & 0.00283\end{array}$

QUAD_TIM,B2 $0.00283 \quad 0.00099$

tau (as correlations)

INTRCPT1,B0 $1.000-0.563$

QUAD_TIM,B2 -0.563 1.000

\begin{tabular}{|c|c|c|c|c|c|}
\hline Fixed Effect & $\begin{array}{c}\text { Standard } \\
\text { Coefficient Erro }\end{array}$ & $\begin{array}{r}\text { Approx. } \\
\text { or } \quad \text { T-ratio }\end{array}$ & d.f. & P-value & \\
\hline $\begin{array}{l}\text { For INTRCPT } \\
\text { INTRCPT2, G0C }\end{array}$ & $\begin{array}{ll}\text { 1, B0 } & \\
0.577792\end{array}$ & 0.041535 & 13.911 & 35 & 0.000 \\
\hline $\begin{array}{l}\text { For TIME slope } \\
\text { INTRCPT2, G10 }\end{array}$ & B1 $\quad 0.019582$ & 0.011791 & 1.661 & 102 & 0.100 \\
\hline For QUAD_TIM s & lope, B2 & & & & \\
\hline $\begin{array}{l}\text { INTRCPT2, G20 } \\
\text { For LG SAA slo }\end{array}$ & pe, B3 ${ }^{-0.015151}$ & 0.011855 & -1.278 & 35 & 0.210 \\
\hline INTRCPT2, G30 & 0.013329 & 0.132117 & 0.101 & 102 & 0.920 \\
\hline
\end{tabular}

Random level-1 coefficient Reliability estimate

$\begin{array}{lc}\text { INTRCPT1, G0 } & 0.896 \\ \text { QUAD_TIM, G2 } & 0.706\end{array}$

The value of the log-likelihood function at iteration $12=7.103355 \mathrm{E}+000$

The outcome variable is LG_CORT

Final estimation of fixed effects: 
$\begin{array}{llllll}\text { INTRCPT2, G40 } & -0.030696 & 0.097449 & -0.315 & 102 & 0.753\end{array}$

For SAAXQ_TI slope, B5

$\begin{array}{llllll}\text { INTRCPT2, G50 } & 0.057450 & 0.059370 & 0.968 & 102 & 0.336\end{array}$

The outcome variable is LG_CORT

Final estimation of variance components:

Random Effect Standard Variance df Chi-square P-value

Deviation Component

$\begin{array}{lllllll}\text { INTRCPT1, u0 } & 0.23124 & 0.05347 & 35 & 365.69491 & 0.000\end{array}$

QUAD_TIM slope, u2 $\quad 0.05443 \quad 0.00296 \quad 35 \quad 126.62201 \quad 0.000$

$\begin{array}{llll}\text { level-1, } & \mathrm{r} & 0.16387 & 0.02685\end{array}$

Statistics for the current model

Deviance $\quad=-14.206709$

Number of estimated parameters $=10$

Model comparison test

Chi-square statistic $=35.10047$

Degrees of freedom $=2$

P-value $\quad=<0.001$

\section{Model 10}

Level-1 Model

LG_CORT $=$ B0 + B $1 *($ TIME $)+$ B2* $($ QUAD_TIM $)+$ B3* $($ LG_SAA $)+B 4 *($ SAAXTIME $)+$ $\mathrm{B} 5 *(\mathrm{SAAX} \overline{\mathrm{X}} \mathrm{TI})+\mathrm{r}$

Level-2 Model

$$
\begin{aligned}
& \mathrm{B} 0=\mathrm{G} 00+\mathrm{G} 01 *(\mathrm{INTERNAL})+\mathrm{u} 0 \\
& \mathrm{~B} 1=\mathrm{G} 10 \\
& \mathrm{~B} 2=\mathrm{G} 20+\mathrm{u} 2 \\
& \mathrm{~B} 3=\mathrm{G} 30 \\
& \mathrm{~B} 4=\mathrm{G} 40 \\
& \mathrm{~B} 5=\mathrm{G} 50
\end{aligned}
$$


LG_SAA has been centered around the group mean.

INTERNAL has been centered around the grand mean.

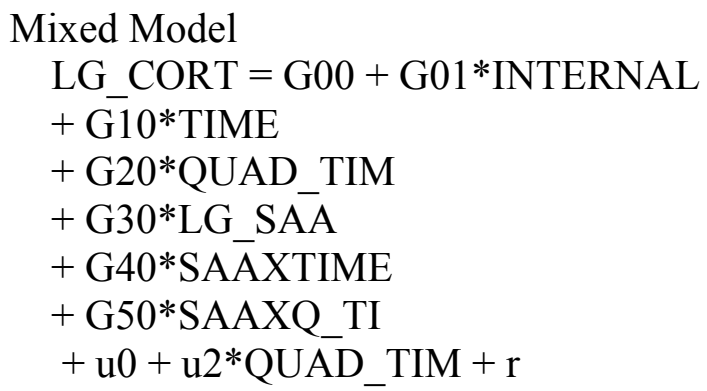

Standard error of $\operatorname{sigma}^{\wedge} 2=\quad 0.00368$

tau

INTRCPT1,B0 $0.05138 \quad-0.00658$

QUAD_TIM,B2 $-0.00658 \quad 0.00296$

Standard errors of tau

INTRCPT1,B0 $0.01354 \quad 0.00275$

QUAD_TIM,B2 $0.00275 \quad 0.00099$

tau (as correlations)

INTRCPT1,B0 $1.000-0.534$

QUAD_TIM,B2 -0.534 1.000

Random level-1 coefficient Reliability estimate

$\begin{array}{lc}\text { INTRCPT1, G0 } & 0.892 \\ \text { QUAD_TIM, G2 } & 0.706\end{array}$

The value of the log-likelihood function at iteration $12=7.404451 \mathrm{E}+000$

The outcome variable is LG_CORT

Final estimation of fixed effects:

Fixed Effect Coefficient Error T-ratio d.f. P-value


For INTRCPT1, B0

$\begin{array}{llllll}\text { INTRCPT2, G00 } & 0.580055 & 0.040924 & 14.174 & 34 & 0.000\end{array}$

$\begin{array}{llllll}\text { INTERNAL, G01 } & -0.005303 & 0.006585 & -0.805 & 34 & 0.426\end{array}$

For TIME slope, B1

$\begin{array}{llllll}\text { INTRCPT2, G10 } & 0.019737 & 0.011788 & 1.674 & 102 & 0.097\end{array}$

For QUAD_TIM slope, B2

$\begin{array}{llllll}\text { INTRCPT2, G20 } & -0.015030 & 0.011847 & -1.269 & 35 & 0.213\end{array}$

For LG_SAA slope, B3

$\begin{array}{llllll}\text { INTRCPT2, G30 } & 0.023748 & 0.133396 & 0.178 & 102 & 0.859\end{array}$

For SAAXTIME slope, B4

$\begin{array}{llllll}\text { INTRCPT2, G40 } & -0.035057 & 0.097444 & -0.360 & 102 & 0.720\end{array}$

For SAAXQ_TI slope, B5

$\begin{array}{llllll}\text { INTRCPT2, G50 } & 0.048527 & 0.060642 & 0.800 & 102 & 0.425\end{array}$

Final estimation of variance components:

Random Effect Standard Variance df Chi-square P-value Deviation Component

$\begin{array}{lllllll}\text { INTRCPT1, u0 } & 0.22668 & 0.05138 & 34 & 352.51665 & 0.000\end{array}$

QUAD_TIM slope, u2 $\quad 0.05437 \quad 0.00296 \quad 35 \quad 126.33625 \quad 0.000$

$\begin{array}{llll}\text { level-1, } & \mathrm{r} & 0.16381 & 0.02684\end{array}$

Statistics for the current model

Deviance $=-14.808903$

Number of estimated parameters $=11$

Model comparison test

Chi-square statistic $=0.60219$

Degrees of freedom $=1$

P-value $\quad=>.500$

\section{Model 11}

Level-1 Model

LG_CORT $=$ B0 + B1*(TIME $)+$ B2* $($ QUAD_TIM $)+B 3 *($ LG_SAA $)+B 4 *($ SAAXTIME $)+$ B5*(SAAXQ_TI $)+r$

Level-2 Model 


$$
\begin{aligned}
& \mathrm{B} 0=\mathrm{G} 00+\mathrm{G} 01 *(\mathrm{INTERNAL})+\mathrm{u} 0 \\
& \mathrm{~B} 1=\mathrm{G} 10+\mathrm{G} 11 *(\mathrm{INTERNAL}) \\
& \mathrm{B} 2=\mathrm{G} 20+\mathrm{G} 21 *(\mathrm{INTERNAL})+\mathrm{u} 2 \\
& \mathrm{~B} 3=\mathrm{G} 30 \\
& \mathrm{~B} 4=\mathrm{G} 40 \\
& \mathrm{~B} 5=\mathrm{G} 50
\end{aligned}
$$

LG_SAA has been centered around the group mean.

INTERNAL has been centered around the grand mean.

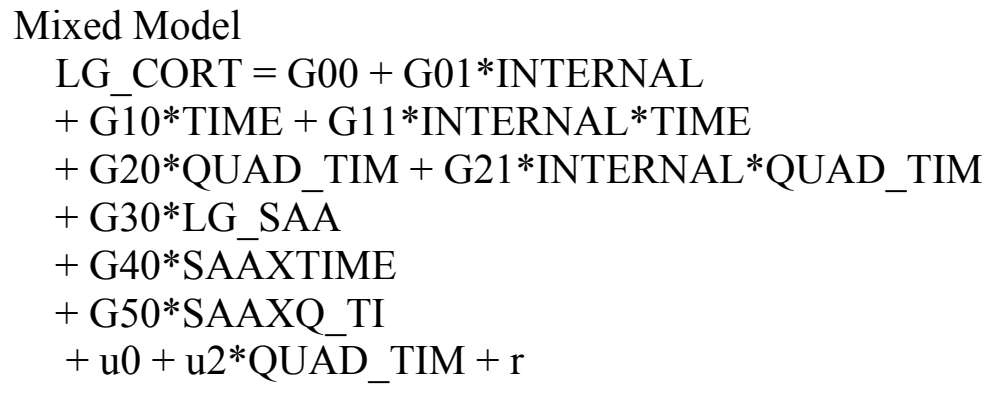

Standard errors of tau

INTRCPT1,B0 $0.01323 \quad 0.00262$

QUAD_TIM,B2 $0.00262 \quad 0.00093$

tau (as correlations)

INTRCPT1,B0 $1.000-0.532$

QUAD_TIM,B2 -0.532 1.000

Random level-1 coefficient Reliability estimate

$\begin{array}{lc}\text { INTRCPT1, G0 } & 0.890 \\ \text { QUAD_TIM, G2 } & 0.686\end{array}$

The value of the log-likelihood function at iteration $12=9.117114 \mathrm{E}+000$

The outcome variable is LG_CORT 
Final estimation of fixed effects:

\begin{tabular}{|c|c|c|c|c|c|}
\hline Fixed Effect & $\begin{array}{c}\text { Standard } \\
\text { Coefficient Erro }\end{array}$ & $\begin{array}{r}\text { Approx. } \\
\text { or } \quad \text { T-ratio }\end{array}$ & d.f. & P-value & \\
\hline \multicolumn{6}{|c|}{ For INTRCPT1, B0 } \\
\hline INTRCPT2, G00 & 0.580562 & $0.040476 \quad 1$ & 14.344 & 34 & 0.000 \\
\hline INTERNAL, G01 & -0.007653 & 0.007097 & -1.078 & 34 & 0.288 \\
\hline \multicolumn{6}{|c|}{ For TIME slope, B1 } \\
\hline INTRCPT2, G10 & 0.021617 & 0.011859 & 1.823 & 101 & 0.071 \\
\hline INTERNAL, G11 & -0.001977 & 0.002070 & -0.955 & 101 & 0.342 \\
\hline \multicolumn{6}{|c|}{ For QUAD_TIM slope, B2 } \\
\hline INTRCPT2, G20 & -0.015758 & 0.011540 & -1.366 & 34 & 0.181 \\
\hline INTERNAL, G21 & 0.002331 & 0.002081 & 1.120 & 34 & 0.270 \\
\hline \multicolumn{6}{|c|}{ For LG_SAA slope, B3 } \\
\hline INTRCPT2, G30 & -0.039184 & 0.136930 & -0.286 & 101 & 0.775 \\
\hline \multicolumn{6}{|c|}{ For SAAXTIME slope, B4 } \\
\hline INTRCPT2, G40 & -0.072500 & 0.100717 & -0.720 & 101 & 0.473 \\
\hline \multicolumn{6}{|c|}{ For SAAXQ_TI slope, B5 } \\
\hline INTRCPT2, G50 & 0.064001 & 0.061317 & 1.044 & 101 & 0.299 \\
\hline
\end{tabular}

Final estimation of variance components:

Random Effect Standard Variance df Chi-square P-value Deviation Component

$\begin{array}{lllllll}\text { INTRCPT1, u0 } & 0.22380 & 0.05009 & 34 & 345.14634 & 0.000\end{array}$ QUAD_TIM slope, u2 $\quad 0.05177 \quad 0.00268 \quad 34 \quad 117.78837 \quad 0.000$ $\begin{array}{llll}\text { level-1, } & \mathrm{r} & 0.16351 & 0.02674\end{array}$

Statistics for the current model

Deviance $\quad=-18.234228$

Number of estimated parameters $=13$

Model comparison test

$\begin{array}{ll}\text { Chi-square statistic } & =3.42532 \\ \begin{array}{c}\text { Degrees of freedom } \\ \text { P-value }\end{array}= & 2 \\ & =0.178\end{array}$

\section{Model 12}


Level-1 Model

LG_CORT $=\mathrm{B} 0+\mathrm{B} 1 *(\mathrm{TIME})+\mathrm{B} 2 *\left(\mathrm{QUAD} \_\mathrm{TIM}\right)+\mathrm{B} 3 *(\mathrm{LG}$ _SAA $)+\mathrm{B} 4 *(\mathrm{SAAXTIME})+$ $\mathrm{B} 5 *\left(\mathrm{SAAX} \mathrm{Q} \_\mathrm{TI}\right)+\mathrm{r}$

Level-2 Model

$$
\begin{aligned}
& \mathrm{B} 0=\mathrm{G} 00+\mathrm{G} 01 *(\mathrm{INTERNAL})+\mathrm{u} 0 \\
& \mathrm{~B} 1=\mathrm{G} 10+\mathrm{G} 11 *(\mathrm{INTERNAL}) \\
& \mathrm{B} 2=\mathrm{G} 20+\mathrm{G} 21 *(\mathrm{INTERNAL})+\mathrm{u} 2 \\
& \mathrm{~B} 3=\mathrm{G} 30+\mathrm{G} 31 *(\mathrm{INTERNAL}) \\
& \mathrm{B} 4=\mathrm{G} 40 \\
& \mathrm{~B} 5=\mathrm{G} 50
\end{aligned}
$$

LG_SAA has been centered around the group mean.

INTERNAL has been centered around the grand mean.

$$
\begin{aligned}
& \text { Mixed Model } \\
& \text { LG_CORT }=\mathrm{G} 00+\text { G0 } 1 * \text { INTERNAL } \\
& \text { + G10*TIME + G11*INTERNAL*TIME } \\
& \text { + G20*QUAD_TIM + G2 } 1 * \text { INTERNAL*QUAD_TIM } \\
& \text { + G30*LG_SAA + G3 } 1 * \text { INTERNAL*LG_SAA } \\
& \text { + G40*SAAXTIME } \\
& \text { + G50*SAAXQ_TI } \\
& \text { + u0 + u2*QUAD_TIM + r }
\end{aligned}
$$

$\operatorname{sigma}^{\wedge} 2=0.02673$

Standard error of $\operatorname{sigma}^{\wedge} 2=\quad 0.00366$

tau

$$
\begin{array}{llc}
\text { INTRCPT1,B0 } & 0.05014 & -0.00613 \\
\text { QUAD_TIM,B2 } & -0.00613 & 0.00267
\end{array}
$$

Standard errors of tau

$$
\begin{array}{lcc}
\text { INTRCPT1,B0 } & 0.01324 & 0.00261 \\
\text { QUAD_TIM,B2 } & 0.00261 & 0.00093
\end{array}
$$

tau (as correlations)

INTRCPT1,B0 $1.000-0.530$

QUAD_TIM,B2 -0.530 1.000

Random level-1 coefficient Reliability estimate 
INTRCPT1, G0 $\quad 0.890$

QUAD_TIM, G2 $\quad 0.686$

The value of the log-likelihood function at iteration $12=9.125267 \mathrm{E}+000$

The outcome variable is LG_CORT

Final estimation of fixed effects:

\begin{tabular}{|c|c|c|c|c|c|}
\hline Fixed Effect & $\begin{array}{c}\text { Standard } \\
\text { Coefficient Erro }\end{array}$ & $\begin{array}{r}\text { Approx. } \\
\text { T-ratio }\end{array}$ & d.f. & P-value & \\
\hline \multicolumn{6}{|c|}{ For INTRCPT1, B0 } \\
\hline INTRCPT2, G00 & 0.580661 & 0.040501 & 14.337 & 34 & 0.000 \\
\hline INTERNAL, G01 & -0.007645 & 0.007100 & -1.077 & 34 & 0.289 \\
\hline \multicolumn{6}{|c|}{ For TIME slope, B1 } \\
\hline INTRCPT2, G10 & 0.021672 & 0.011863 & 1.827 & 100 & 0.071 \\
\hline INTERNAL, G11 & -0.001997 & 0.002076 & -0.962 & 100 & 0.338 \\
\hline \multicolumn{6}{|c|}{ For QUAD_TIM slope, B2 } \\
\hline INTRCPT 2, G20 & -0.015610 & 0.011585 & -1.347 & 34 & 0.187 \\
\hline INTERNAL, G21 & 0.002322 & 0.002080 & 1.116 & 34 & 0.272 \\
\hline \multicolumn{6}{|c|}{ For LG_SAA slope, B3 } \\
\hline INTRCPT2, G30 & -0.039486 & 0.136984 & -0.288 & 100 & 0.774 \\
\hline INTERNAL, G3 & -0.002679 & 0.020792 & -0.129 & 100 & 0.898 \\
\hline \multicolumn{6}{|c|}{ For SAAXTIME slope, B4 } \\
\hline INTRCPT2, G40 & -0.074246 & 0.101567 & -0.731 & 100 & 0.466 \\
\hline \multicolumn{6}{|c|}{ For SAAXQ_TI slope, B5 } \\
\hline INTRCPT2, G50 & 0.062625 & 0.062174 & 1.007 & 100 & 0.316 \\
\hline
\end{tabular}

Final estimation of variance components:

Random Effect Standard Variance df Chi-square P-value Deviation Component

$\begin{array}{lllllll}\text { INTRCPT1, u0 } & 0.22392 & 0.05014 & 34 & 345.58804 & 0.000\end{array}$

QUAD_TIM slope, u2 $\quad 0.05170 \quad 0.00267 \quad 34 \quad 117.55099 \quad 0.000$ $\begin{array}{llll}\text { level-1, } & \mathrm{r} & 0.16348 & 0.02673\end{array}$

Statistics for the current model

Deviance $\quad=-18.250533$

Number of estimated parameters $=14$ 
Model comparison test

Chi-square statistic $=0.01631$

Degrees of freedom $=1$

P-value $\quad=\quad>.500$

\section{Model 13}

Level-1 Model

LG_CORT $=\mathrm{B} 0+\mathrm{B} 1 *(\mathrm{TIME})+\mathrm{B} 2 *\left(\mathrm{QUAD} \_\mathrm{TIM}\right)+\mathrm{B} 3 *\left(\mathrm{LG} \_\mathrm{SAA}\right)+\mathrm{B} 4 *(\mathrm{SAAXTIME})+$ $\mathrm{B} 5 *(\mathrm{SAAXQ}$ TI $)+\mathrm{r}$

Level-2 Model

$$
\begin{aligned}
& \mathrm{B} 0=\mathrm{G} 00+\mathrm{G} 01 *(\text { INTERNAL })+\mathrm{u} 0 \\
& \mathrm{~B} 1=\mathrm{G} 10+\mathrm{G} 11 *(\text { INTERNAL }) \\
& \mathrm{B} 2=\mathrm{G} 20+\mathrm{G} 21 *(\text { INTERNAL })+\mathrm{u} 2 \\
& \mathrm{~B} 3=\mathrm{G} 30+\mathrm{G} 31 *(\text { INTERNAL }) \\
& \mathrm{B} 4=\mathrm{G} 40+\mathrm{G} 41 *(\text { INTERNAL }) \\
& \mathrm{B} 5=\mathrm{G} 50+\mathrm{G} 51 *(\text { INTERNAL })
\end{aligned}
$$

LG_SAA has been centered around the group mean.

INTERNAL has been centered around the grand mean.

$$
\begin{aligned}
& \text { Mixed Model } \\
& \text { LG_CORT }=\mathrm{G} 00+\text { G01*INTERNAL } \\
& \text { + G10*TIME + G11*INTERNAL*TIME } \\
& \text { + G20*QUAD_TIM + G21*INTERNAL*QUAD_TIM } \\
& \text { + G30*LG_SAA + G31*INTERNAL*LG_SAA } \\
& \text { + G40*SAAXTIME + G41*INTERNAL*SAAXTIME } \\
& \text { + G50*SAAXQ_TI + G51*INTERNAL*SAAXQ_TI } \\
& \text { + u0 + u2*QUAD_TIM + r }
\end{aligned}
$$

$\operatorname{sigma}^{\wedge} 2=0.02643$

Standard error of $\operatorname{sigma}^{\wedge} 2=0.00362$

$$
\begin{array}{lcc}
\text { tau } & & \\
\text { INTRCPT1,B0 } & 0.04914 & -0.00652 \\
\text { QUAD_TIM,B2 } & -0.00652 & 0.00286
\end{array}
$$

Standard errors of tau

INTRCPT1,B0 $0.01299 \quad 0.00266$ 
QUAD_TIM,B2 $0.00266 \quad 0.00096$

tau (as correlations)

INTRCPT1,B0 $1.000-0.550$

QUAD_TIM,B2 -0.550 1.000

Random level-1 coefficient Reliability estimate

$\begin{array}{lc}\text { INTRCPT1, G0 } & 0.889 \\ \text { QUAD_TIM, G2 } & 0.702\end{array}$

The value of the log-likelihood function at iteration $13=9.626734 \mathrm{E}+000$

The outcome variable is LG_CORT

Final estimation of fixed effects:

\begin{tabular}{|c|c|c|c|c|c|}
\hline Fixed Effect & $\begin{array}{c}\text { Standard } \\
\text { Coefficient Erro }\end{array}$ & $\begin{array}{r}\text { Approx. } \\
\text { or } \quad \text { T-ratio }\end{array}$ & d.f. & P-value & \\
\hline \multicolumn{6}{|c|}{ For INTRCPT1, B0 } \\
\hline INTRCPT2, G00 & 0.577496 & 0.040249 & 14.348 & 34 & 0.000 \\
\hline INTERNAL, G01 & 007983 & 0.007051 & & 34 & 0.266 \\
\hline \multicolumn{6}{|c|}{ For TIME slope, B1 } \\
\hline INTRCPT2, G10 & 0.022661 & 0.012113 & 1.871 & 98 & 0.064 \\
\hline INTERNAL, G11 & 896 & 0.0 & -0 & 98 & 0.367 \\
\hline \multicolumn{6}{|c|}{ For QUAD_TIM slope, B2 } \\
\hline INTRCPT̄2, G20 & -0.018135 & 0.01 & -1.5 & 34 & 0.141 \\
\hline INTERNAL, G21 & 0.001630 & 0.00 & 0.7 & 34 & 0.467 \\
\hline \multicolumn{6}{|c|}{ For LG_SAA slope, B3 } \\
\hline INTRCPT2, G30 & -0.04 & 0.13 & -0.3 & 98 & 0.764 \\
\hline INTERNAL, G31 & 369 & 0.02 & 0.230 & 98 & 0.819 \\
\hline \multicolumn{6}{|c|}{ For SAAXTIME slope, B4 } \\
\hline INTRCPT2, G40 & -0.073310 & 0.101 & -0.724 & 98 & 0.471 \\
\hline INTERNAL, G41 & 0.000818 & 0.017453 & 0.047 & 98 & 0.963 \\
\hline \multicolumn{6}{|c|}{ For SAAXQ_TI slope, B5 } \\
\hline INTRCPT2, G50 & 0.055921 & 0.062509 & 0.8 & 98 & 0.373 \\
\hline INTERNAL, G51 & -0.008443 & 0.010798 & -0.782 & 98 & 0.436 \\
\hline
\end{tabular}

Final estimation of variance components:

Random Effect Standard Variance df Chi-square P-value Deviation Component 
$\begin{array}{lllllll}\text { INTRCPT1, u0 } & 0.22167 & 0.04914 & 34 & 342.49175 & 0.000\end{array}$

QUAD_TIM slope, u2 $\quad 0.05344 \quad 0.00286 \quad 34 \quad 124.14538 \quad 0.000$

$\begin{array}{llll}\text { level-1, } & \mathrm{r} & 0.16258 & 0.02643\end{array}$

Statistics for the current model

Deviance $\quad=-19.253467$

Number of estimated parameters $=16$

Model comparison test

Chi-square statistic $=1.00293$

Degrees of freedom $=2$

P-value $\quad=\quad>.500$

\section{Model 14}

Level-1 Model

$$
\text { LG_CORT }=\text { B0 }+ \text { B1 } *(\text { TIME })+\text { B2 } *\left(Q U A D \_T I M\right)+B 3 *\left(L G \_S A A\right)+r
$$

Level-2 Model

$$
\begin{aligned}
& \mathrm{B} 0=\mathrm{G} 00+\mathrm{G} 01 *(\text { INTERNAL })+\mathrm{u} 0 \\
& \mathrm{~B} 1=\mathrm{G} 10 \\
& \mathrm{~B} 2=\mathrm{G} 20+\mathrm{u} 2 \\
& \mathrm{~B} 3=\mathrm{G} 30
\end{aligned}
$$

INTERNAL has been centered around the grand mean.

$$
\begin{aligned}
& \text { Mixed Model } \\
& \text { LG_CORT }=\mathrm{G} 00+\mathrm{G} 01 * \text { INTERNAL } \\
& + \text { G10*TIME } \\
& + \text { G20*QUAD_TIM } \\
& + \text { G30*LG_SAA } \\
& +\mathrm{u} 0+\mathrm{u} 2 * \text { QUAD_TIM }+\mathrm{r}
\end{aligned}
$$

$\operatorname{sigma}^{\wedge} 2=0.02683$

Standard error of sigma ${ }^{\wedge} 2=0.00368$

tau

$\begin{array}{lll}\text { INTRCPT1,B0 } & 0.05326 & -0.00658\end{array}$ 
QUAD_TIM,B2 $\quad-0.00658 \quad 0.00307$

Standard errors of tau

$\begin{array}{lll}\text { INTRCPT1,B0 } & 0.01399 & 0.00282\end{array}$

$\begin{array}{lll}\text { QUAD_TIM,B2 } & 0.00282 & 0.00102\end{array}$

tau (as correlations)

INTRCPT1,B0 $1.000-0.515$

QUAD_TIM,B2 -0.515 1.000

Random level-1 coefficient Reliability estimate

\begin{tabular}{lc} 
INTRCPT1, G0 & 0.895 \\
QUAD_TIM, G2 & 0.714 \\
\hline------
\end{tabular}

The value of the log-likelihood function at iteration $9=6.175171 \mathrm{E}+000$

The outcome variable is LG_CORT

Final estimation of fixed effects:

\begin{tabular}{|c|c|c|c|c|c|}
\hline Fixed Effect & $\begin{array}{l}\text { Standard } \\
\text { Coefficient Error }\end{array}$ & $\begin{array}{r}\text { Approx. } \\
\text { T-ratio }\end{array}$ & d.f. & P-value & \\
\hline \multicolumn{6}{|c|}{ For $\quad$ INTRCPT1, B0 } \\
\hline INTRCPT2, G00 & $0.500946 \quad 0$ & 0.162670 & 3.080 & 34 & 0.004 \\
\hline INTERNAL, G01 & -0.007410 & 0.006800 & -1.090 & 34 & 0.284 \\
\hline \multicolumn{6}{|c|}{ For TIME slope, B1 } \\
\hline INTRCPT2, G10 & $0.017406 \quad 0$ & 0.011413 & 1.525 & 104 & 0.130 \\
\hline \multicolumn{6}{|c|}{ For QUAD_TIM slope, B2 } \\
\hline INTRCPT2, G20 & $-0.016026 \quad 0$ & 0.011925 & -1.344 & 35 & 0.188 \\
\hline \multicolumn{6}{|c|}{ For LG_SAA slope, B3 } \\
\hline INTRCPT2, G30 & 0.0466210 & 0.089924 & 0.518 & 104 & 0.605 \\
\hline
\end{tabular}

Final estimation of variance components:

Random Effect Standard Variance df Chi-square P-value Deviation Component

$\begin{array}{lllllll}\text { INTRCPT1， u0 } & 0.23079 & 0.05326 & 34 & 364.43868 & 0.000\end{array}$

$\begin{array}{llllll}\text { QUAD_TIM slope, u2 } & 0.05541 & 0.00307 & 35 & 129.89187 & 0.000\end{array}$ $\begin{array}{llll}\text { level-1, } & \mathrm{r} & 0.16381 & 0.02683\end{array}$ 
Statistics for the current model

Deviance $\quad=-12.350342$

Number of estimated parameters $=9$

Model comparison test

Chi-square statistic $=1.13796$

Degrees of freedom $=1$

P-value $\quad=0.286$

\section{Model 15}

Level-1 Model

$$
\text { LG_CORT }=\text { B0 + B1 } *(\text { TIME })+\text { B2* }\left(\mathrm{QUAD} \_ \text {TIM }\right)+r
$$

Level-2 Model

$$
\begin{aligned}
& \mathrm{B} 0=\mathrm{G} 00+\mathrm{u} 0 \\
& \mathrm{~B} 1=\mathrm{G} 10 \\
& \mathrm{~B} 2=\mathrm{G} 20+\mathrm{u} 2
\end{aligned}
$$

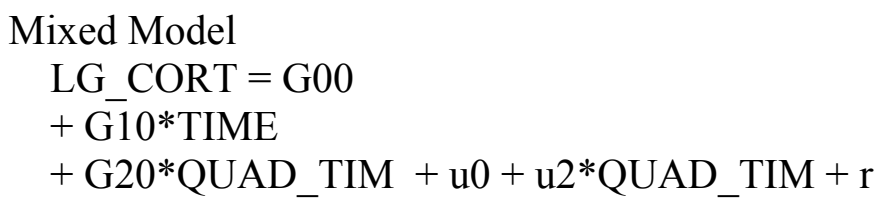

$\operatorname{sigma}^{\wedge} 2=0.03125$

Standard error of $\operatorname{sigma}^{\wedge} 2=0.00350$

tau

INTRCPT1,B0 $\quad 0.06080 \quad-0.00648$

$\begin{array}{lll}\text { QUAD_TIM,B2 } & -0.00648 & 0.00159\end{array}$

Standard errors of tau

$\begin{array}{lcc}\text { INTRCPT1,B0 } & 0.01478 & 0.00227 \\ \text { QUAD_TIM,B2 } & 0.00227 & 0.00056\end{array}$

tau (as correlations)

INTRCPT1,B0 $1.000-0.658$

QUAD_TIM,B2 -0.658 1.000 
Random level-1 coefficient Reliability estimate

$\begin{array}{lc}\text { INTRCPT1, G0 } & 0.921 \\ \text { QUAD_TIM, G2 } & 0.646\end{array}$

The value of the log-likelihood function at iteration $9=1.052137 \mathrm{E}+001$

The outcome variable is LG_CORT

Final estimation of fixed effects:

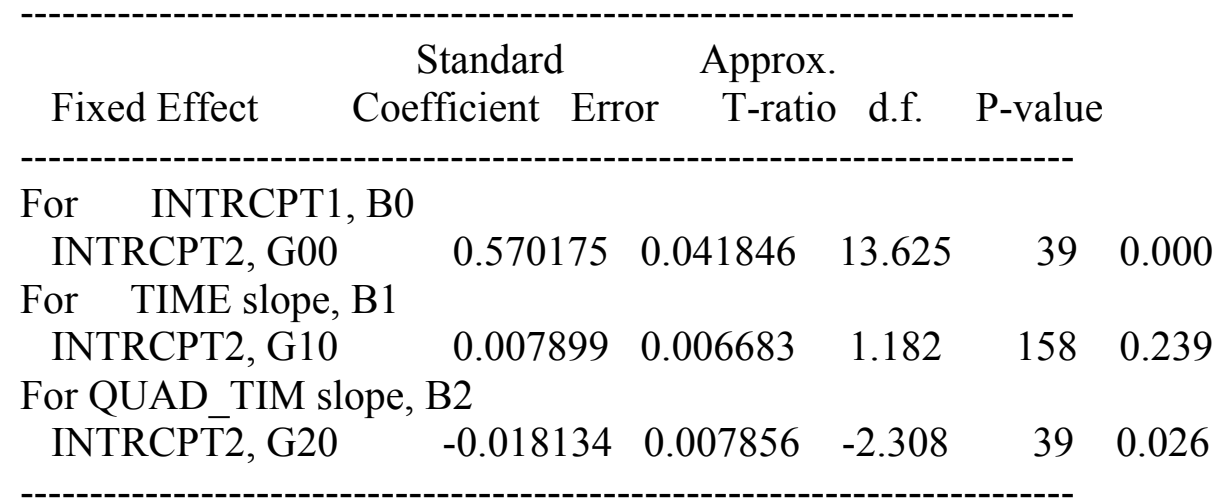

Final estimation of variance components:

Random Effect Standard Variance df Chi-square P-value
Deviation Component

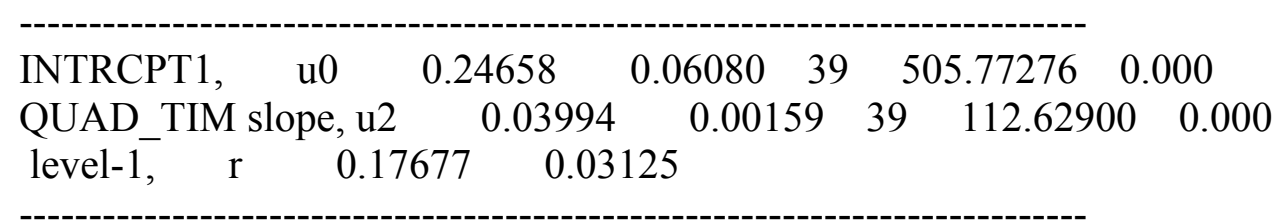

Statistics for the current model

Deviance $\quad=-21.042742$

Number of estimated parameters $=7$

Model comparison test

Chi-square statistic $=31.97181$

Degrees of freedom $=2$

$\mathrm{P}$-value $\quad=\quad<0.001$ 


\section{Model 16}

Level-1 Model

$$
\text { LG_CORT }=\mathrm{B} 0+\mathrm{B} 1 *(\mathrm{TIME})+\mathrm{B} 2 *\left(\mathrm{QUAD} \_\mathrm{TIM}\right)+\mathrm{r}
$$

Level-2 Model

$$
\begin{aligned}
& \mathrm{B} 0=\mathrm{G} 00+\mathrm{G} 01 *(\mathrm{INTERNAL})+\mathrm{u} 0 \\
& \mathrm{~B} 1=\mathrm{G} 10 \\
& \mathrm{~B} 2=\mathrm{G} 20+\mathrm{u} 2
\end{aligned}
$$

INTERNAL has been centered around the grand mean.

$$
\begin{aligned}
& \text { Mixed Model } \\
& \text { LG_CORT }=\mathrm{G} 00+\mathrm{G} 01 * \text { INTERNAL } \\
& + \text { G10*TIME } \\
& + \text { G20*QUAD_TIM } \\
& \quad+\mathrm{u} 0+\mathrm{u} 2 * \text { QUAD_TIM }+\mathrm{r}
\end{aligned}
$$

$\operatorname{sigma}^{\wedge} 2=0.03125$

Standard error of $\operatorname{sigma}^{\wedge} 2=\quad 0.00350$

tau

INTRCPT1,B0 $0.05887 \quad-0.00638$

QUAD_TIM,B2 $-0.00638 \quad 0.00160$

Standard errors of tau

$\begin{array}{lll}\text { INTRCPT1,B0 } 0.01435 & 0.00223\end{array}$

QUAD_TIM,B2 $0.00223 \quad 0.00056$

tau (as correlations)

INTRCPT1,B0 $1.000-0.658$

QUAD_TIM,B2 -0.658 1.000

Random level-1 coefficient Reliability estimate

$\begin{array}{lc}\text { INTRCPT1, G0 } & 0.918 \\ \text { QUAD_TIM, G2 } & 0.646\end{array}$

The value of the log-likelihood function at iteration $9=1.109741 \mathrm{E}+001$

\section{Model 17}


Level-1 Model

$$
\text { LG_CORT }=\text { B } 0+\text { B } 1 *(T I M E)+B 2 *\left(Q U A D \_T I M\right)+r
$$

Level-2 Model

$$
\mathrm{B} 0=\mathrm{G} 00+\mathrm{G} 01 *(\mathrm{CHILD} E \mathrm{EA})+\mathrm{G} 02 *(\mathrm{CHILD} \text {-WA })+\mathrm{G} 03 *\left(\mathrm{CHILD} \_\mathrm{SE}\right)+
$$
G04*(CHILD_AG)

+ G05*(ETHNICIT $)+$ G06*(TIME_GAP $)+u 0$

$\mathrm{B} 1=\mathrm{G} 10$

$\mathrm{B} 2=\mathrm{G} 20+\mathrm{u} 2$

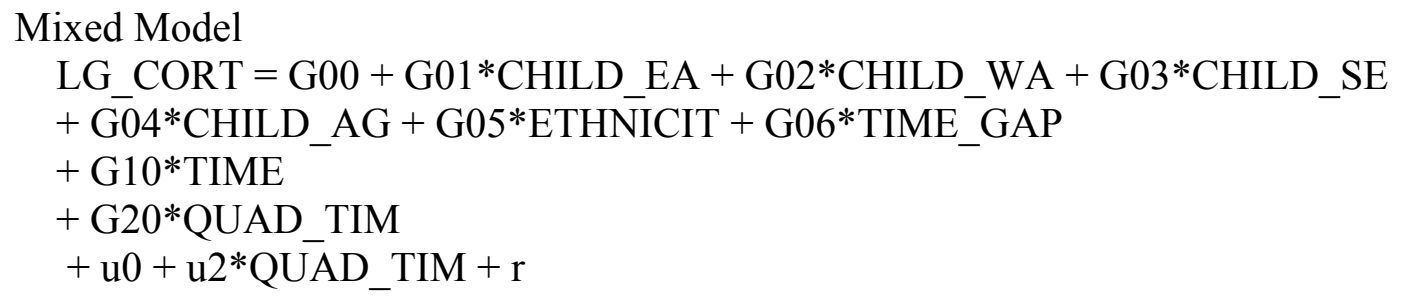

Standard errors of tau

$\begin{array}{lll}\text { INTRCPT1,B0 } & 0.01361 & 0.00216\end{array}$

QUAD_TIM,B2 $0.00216 \quad 0.00056$

tau (as correlations)

INTRCPT1,B0 $1.000-0.641$

QUAD_TIM,B2 -0.641 1.000

Random level-1 coefficient Reliability estimate

$\begin{array}{lc}\text { INTRCPT1, G0 } & 0.914 \\ \text { QUAD_TIM, G2 } & 0.646\end{array}$

The value of the log-likelihood function at iteration $17=1.175513 \mathrm{E}+001$

Final estimation of fixed effects:

Standard Approx. 
Fixed Effect Coefficient Error T-ratio d.f. P-value

$\begin{array}{lccccc}\text { For INTRCPT1, B0 } & & & & & \\ \text { INTRCPT2, G00 } & 0.577469 & 0.576548 & 1.002 & 33 & 0.324 \\ \text { CHILD_EA, G01 } & 0.000007 & 0.000009 & 0.748 & 33 & 0.460 \\ \text { CHILD_WA, G02 } & 0.000001 & 0.000009 & 0.142 & 33 & 0.888 \\ \text { CHILD_SE, G03 } & 0.052238 & 0.069870 & 0.748 & 33 & 0.460 \\ \text { CHILD_AG, G04 } & -0.035407 & 0.054001 & -0.656 & 33 & 0.517 \\ \text { ETHNICIT, G05 } & -0.008395 & 0.013396 & -0.627 & 33 & 0.535 \\ \quad \text { TIME_GAP, G06 } & -0.004146 & 0.016195 & -0.256 & 33 & 0.800 \\ \text { For TIME slope, B1 } & & & & & \\ \quad \text { INTRCPT2, G10 } & 0.007894 & 0.006682 & 1.181 & 158 & 0.239 \\ \text { For QUAD_TIM slope, B2 } & & & & & \\ \text { INTRCPT2, G20 } & -0.018124 & 0.007859 & -2.306 & 39 & 0.027\end{array}$

Final estimation of variance components:

Random Effect Standard Variance df Chi-square P-value Deviation Component

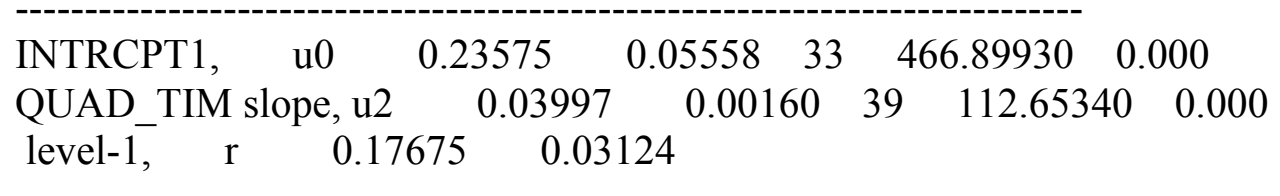

Statistics for the current model

Deviance $\quad=-23.510258$

Number of estimated parameters $=13$

Model comparison test

$\begin{array}{ll}\text { Chi-square statistic }= & 2.44207 \\ \text { Degrees of freedom } & =5 \\ \text { P-value } & =\quad>.500\end{array}$

\section{Model 18- Final model}

Level-1 Model

$$
\text { LG_CORT }=\text { B0 + B1* (TIME })+ \text { B2* }(\text { QUAD_TIM })+r
$$

Level-2 Model

$$
\mathrm{B} 0=\mathrm{G} 00+\mathrm{u} 0
$$




$$
\begin{aligned}
& \mathrm{B} 1=\mathrm{G} 10 \\
& \mathrm{~B} 2=\mathrm{G} 20+\mathrm{u} 2
\end{aligned}
$$

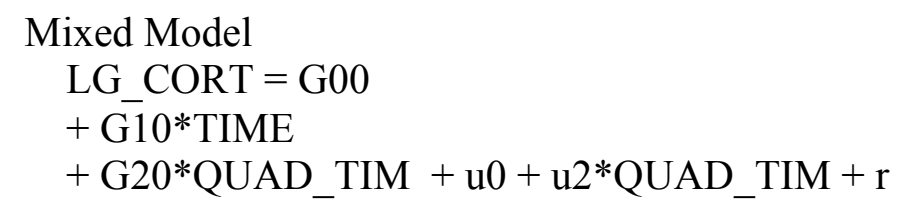

$\operatorname{sigma}^{\wedge} 2=0.03144$

tau

INTRCPT1,B0 $0.06246 \quad-0.00665$

QUAD_TIM,B2 $-0.00665 \quad 0.00165$

tau (as correlations)

INTRCPT1,B0 $1.000-0.654$

QUAD_TIM,B2 -0.654 1.000

Random level-1 coefficient Reliability estimate

\begin{tabular}{lc}
\hline INTRCPT1, G0 & 0.922 \\
QUAD_TIM, G2 & 0.653
\end{tabular}

The value of the log-likelihood function at iteration $15=-8.358201 \mathrm{E}-001$

The outcome variable is LG_CORT

Final estimation of fixed effects:

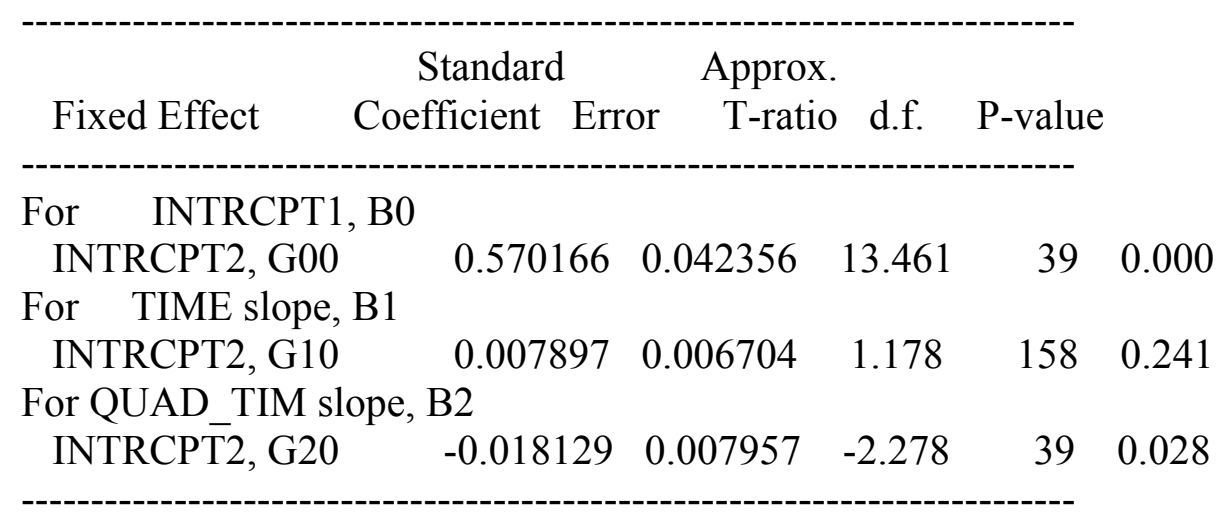

Final estimation of variance components:

Random Effect Standard Variance df Chi-square P-value Deviation Component 


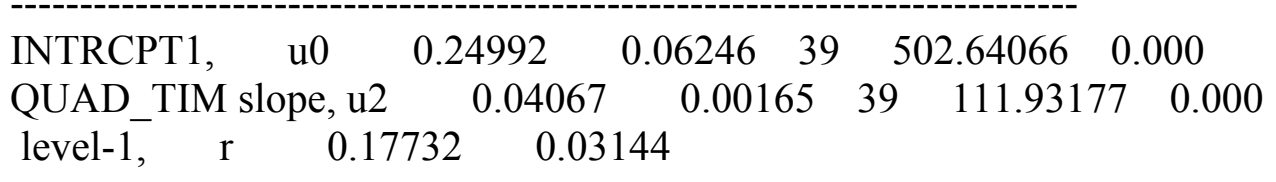

Statistics for current covariance components model

Deviance $\quad=1.671640$

Number of estimated parameters $=4$

Variance-Covariance components test

Chi-square statistic $=22.71438$

Degrees of freedom $=3$

$\mathrm{P}$-value $\quad=\quad<0.001$ 


\section{Appendix 4.3}

\section{Model Equations and Results: Child-Reported Depression in the Competition Challenge}

\section{Model 1}

Level-1 Model

LG_CORT $=\mathrm{B} 0+\mathrm{r}$

Level-2 Model

$\mathrm{B} 0=\mathrm{G} 00+\mathrm{u} 0$

Mixed Model

$\mathrm{LG} \_\mathrm{CORT}=\mathrm{G} 00+\mathrm{u} 0+\mathrm{r}$

$\operatorname{sigma}^{\wedge} 2=0.02518$

Standard error of $\operatorname{sigma}^{\wedge} 2=0.00235$

tau

INTRCPT1,B0 $\quad 0.04528$

Standard error of tau

INTRCPT1,B0 0.01032

Random level-1 coefficient Reliability estimate

INTRCPT1, G0 $\quad 0.915$

The value of the log-likelihood function at iteration $2=5.970427 \mathrm{E}+001$

The outcome variable is LG_CORT

Final estimation of fixed effects:

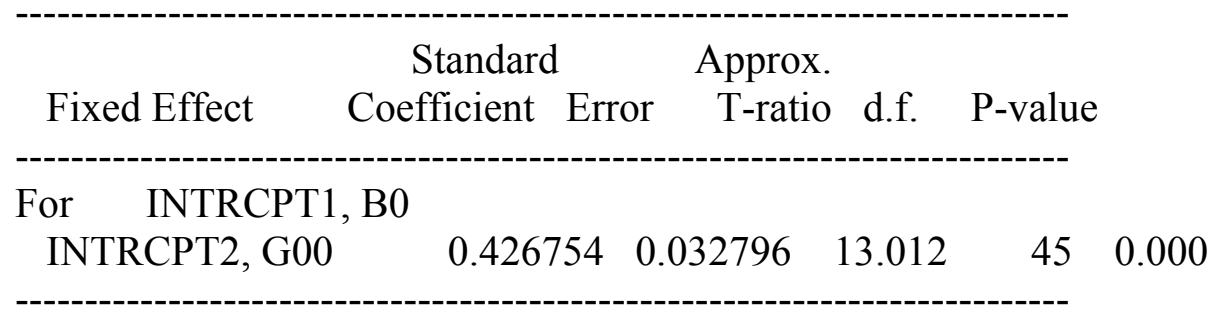

Final estimation of variance components: 

Random Effect Standard Variance df Chi-square P-value
Deviation Component

$\begin{array}{lllllll}\text { INTRCPT1, u0 } & 0.21279 & 0.04528 & 45 & 542.32638 & 0.000\end{array}$
$\begin{array}{llll}\text { level-1, } & \mathrm{r} & 0.15868 & 0.02518\end{array}$

Statistics for the current model

Deviance $\quad=-119.408532$

Number of estimated parameters $=3$

\section{Model 2}

Level-1 Model

$$
\text { LG_CORT }=\mathrm{B} 0+\mathrm{B} 1 *(\mathrm{TIME})+\mathrm{r}
$$

Level-2 Model

$$
\begin{aligned}
& \mathrm{B} 0=\mathrm{G} 00+\mathrm{u} 0 \\
& \mathrm{~B} 1=\mathrm{G} 10
\end{aligned}
$$

$$
\begin{aligned}
& \text { Mixed Model } \\
& \text { LG_CORT }=\text { G00 } \\
& + \text { G10*TIME }+\mathrm{u} 0+\mathrm{r}
\end{aligned}
$$

$\operatorname{sigma}^{\wedge} 2=0.02518$

Standard error of $\operatorname{sigma}^{\wedge} 2=0.00235$

tau

INTRCPT1,B0 0.04528

Standard error of tau

INTRCPT1,B0 0.01032

Random level-1 coefficient Reliability estimate

INTRCPT1, G0 $\quad 0.915$

The value of the log-likelihood function at iteration $2=5.970483 \mathrm{E}+001$

The outcome variable is LG_CORT 
Final estimation of fixed effects:

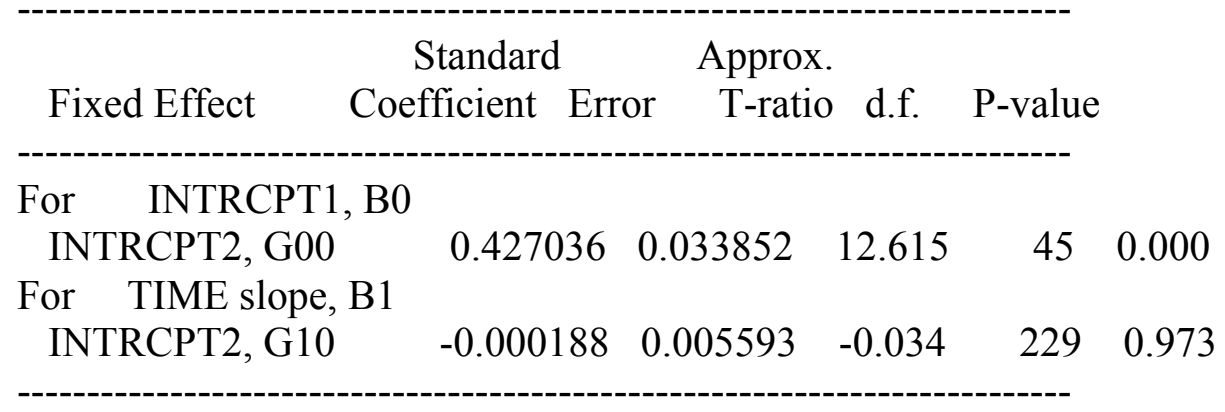

Final estimation of variance components:

Random Effect Standard Variance df Chi-square P-value Deviation Component

\begin{tabular}{|c|c|c|c|}
\hline INTRCPT1, & 0.21279 & $0.04528 \quad 45$ & 542.32904 \\
\hline level-1, $\quad r$ & $0.15868 \quad 0$ & 2518 & \\
\hline
\end{tabular}

Statistics for the current model

Deviance $\quad=-119.409659$

Number of estimated parameters $=4$

Model comparison test

$\begin{array}{ll}\text { Chi-square statistic } & =0.00113 \\ \text { Degrees of freedom } & =1 \\ \text { P-value }= & >.500\end{array}$

\section{Model 3}

Level-1 Model

$$
\text { LG_CORT }=\text { B0 }+ \text { B } 1 *(\text { TIME })+\text { B2 } *\left(Q U A D \_T I M\right)+r
$$

Level-2 Model

$$
\begin{aligned}
& \mathrm{B} 0=\mathrm{G} 00+\mathrm{u} 0 \\
& \mathrm{~B} 1=\mathrm{G} 10 \\
& \mathrm{~B} 2=\mathrm{G} 20
\end{aligned}
$$

Mixed Model

LG_CORT $=$ G00 
+ G10*TIME

$+\mathrm{G} 20 * \mathrm{QUAD}$ TIM $+\mathrm{u} 0+\mathrm{r}$

$\operatorname{sigma}^{\wedge} 2=0.02376$

Standard error of $\operatorname{sigma}^{\wedge} 2=0.00222$

tau

INTRCPT1,B0 0.04552

Standard error of tau

INTRCPT1,B0 0.01032

Random level-1 coefficient Reliability estimate

INTRCPT1, G0 $\quad 0.920$

The value of the log-likelihood function at iteration $2=6.637930 \mathrm{E}+001$

The outcome variable is LG_CORT

Final estimation of fixed effects:

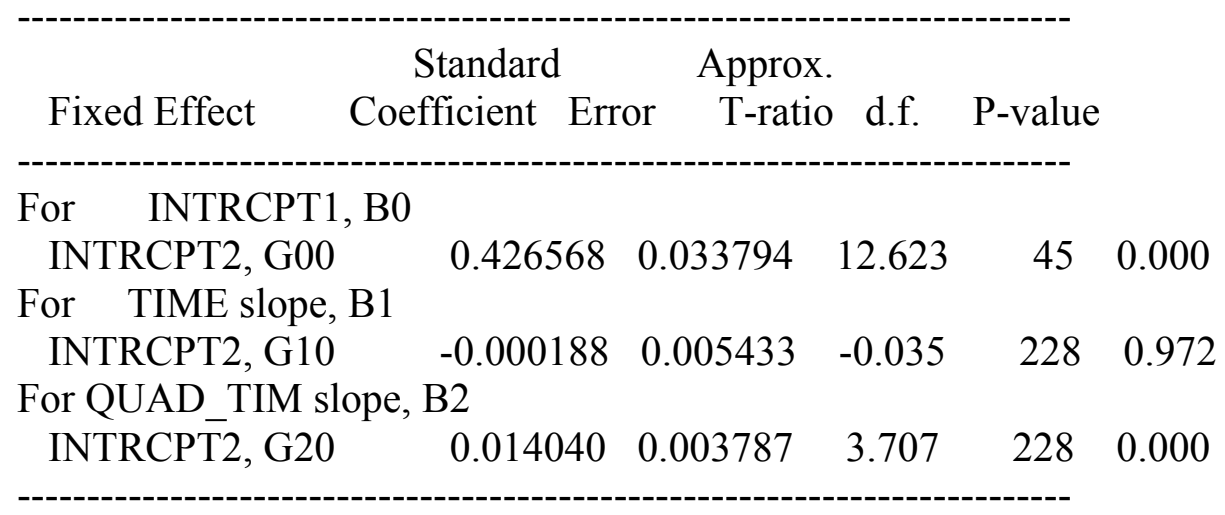

Final estimation of variance components:

Random Effect Standard Variance df Chi-square P-value Deviation Component

$\begin{array}{lllllll}\text { INTRCPT1, } & \text { u0 } & 0.21335 & 0.04552 & 45 & 574.73653 & 0.000 \\ \text { level-1, r } & 0.15414 & 0.02376 & & & \end{array}$

Statistics for the current model 
Deviance $\quad=-132.758591$

Number of estimated parameters $=5$

Model comparison test

Chi-square statistic $=13.34893$

Degrees of freedom $=1$

P-value $\quad=\quad<0.001$

\section{Model 4}

Level-1 Model

$$
\text { LG_CORT }=\mathrm{B} 0+\mathrm{B} 1 *(\mathrm{TIME})+\mathrm{B} 2 *\left(\mathrm{QUAD} \_\mathrm{TIM}\right)+\mathrm{B} 3 *\left(\mathrm{LG} \_\mathrm{SAA}\right)+\mathrm{r}
$$

Level-2 Model

$$
\begin{aligned}
& \mathrm{B} 0=\mathrm{G} 00+\mathrm{u} 0 \\
& \mathrm{~B} 1=\mathrm{G} 10 \\
& \mathrm{~B} 2=\mathrm{G} 20 \\
& \mathrm{~B} 3=\mathrm{G} 30
\end{aligned}
$$

LG_SAA has been centered around the group mean.

$$
\begin{aligned}
& \text { Mixed Model } \\
& \text { LG_CORT = G00 } \\
& + \text { G10*TIME } \\
& + \text { G20*QUAD_TIM } \\
& + \text { G30*LG_SAA } \\
& +\mathrm{u} 0+\mathrm{r}
\end{aligned}
$$

$\operatorname{sigma}^{\wedge} 2=0.02400$

Standard error of $\operatorname{sigma}^{\wedge} 2=\quad 0.00263$

tau

INTRCPT1,B0 0.03355

Standard error of tau

INTRCPT1,B0 0.00839

Random level-1 coefficient Reliability estimate

INTRCPT1, G0 0.874


The value of the log-likelihood function at iteration $4=5.055731 \mathrm{E}+001$

The outcome variable is LG_CORT

Final estimation of fixed effects:

\begin{tabular}{|c|c|c|c|c|c|}
\hline Fixed Effect & $\begin{array}{c}\text { Standard } \\
\text { Coefficient Err }\end{array}$ & $\begin{array}{r}\text { Approx. } \\
\text { or } \quad \text { T-ratio }\end{array}$ & d.f. & P-value & \\
\hline \multicolumn{6}{|c|}{ For INTRCPT1, B0 } \\
\hline INTRCPT2, G00 & 0.447353 & 0.031298 & 14.293 & 41 & 0.000 \\
\hline \multicolumn{6}{|c|}{ For TIME slope, B1 } \\
\hline INTRCPT2, G10 & -0.003697 & 0.009949 & -0.372 & 164 & 0.71 \\
\hline \multicolumn{6}{|c|}{ For QUAD_TIM slope, B2 } \\
\hline INTRCPT2, G20 & 0.012138 & 0.006513 & 1.864 & 164 & 0.064 \\
\hline \multicolumn{6}{|c|}{ For_LG_SAA slope, B3 } \\
\hline INTRCי-PT2, G30 & 0.069475 & 0.104917 & 0.662 & 164 & 0.509 \\
\hline
\end{tabular}

Final estimation of variance components:

Random Effect Standard Variance df Chi-square P-value Deviation Component

$\begin{array}{lllllll}\text { INTRCPT1, u0 } & 0.18317 & 0.03355 & 41 & 335.27577 & 0.000\end{array}$
level-1, $\quad$ r $\quad 0.15493 \quad 0.02400$

Statistics for the current model

Deviance $\quad=-101.114613$

Number of estimated parameters $=6$

Model comparison test

Chi-square statistic $=31.64398$

Degrees of freedom $=1$

P-value $\quad=\quad<0.001$

\section{Model 5}

Level-1 Model

$$
\text { LG_CORT }=\text { B0 }+ \text { B } 1 *(T I M E)+B 2 *\left(Q U A D \_T I M\right)+B 3 *\left(L G \_S A A\right)+B 4 *\left(S A A X Q \_T I\right)+r
$$

Level-2 Model

$$
\mathrm{B} 0=\mathrm{G} 00+\mathrm{u} 0
$$




$$
\begin{aligned}
& \mathrm{B} 1=\mathrm{G} 10 \\
& \mathrm{~B} 2=\mathrm{G} 20 \\
& \mathrm{~B} 3=\mathrm{G} 30 \\
& \mathrm{~B} 4=\mathrm{G} 40
\end{aligned}
$$

LG_SAA has been centered around the group mean.

$$
\begin{aligned}
& \text { Mixed Model } \\
& \text { LG_CORT = G00 } \\
& + \text { G10*TIME } \\
& + \text { G20*QUAD_TIM } \\
& \text { + G30*LG_SAA } \\
& \text { + G40*SAAXQ_TI } \\
& +\mathrm{u} 0+\mathrm{r}
\end{aligned}
$$

$\operatorname{sigma}^{\wedge} 2=0.02402$

\begin{tabular}{|c|c|c|c|c|c|}
\hline Fixed Effect & $\begin{array}{c}\text { Standard } \\
\text { Coefficient Err }\end{array}$ & $\begin{array}{r}\text { Approx. } \\
\text { or } \quad \text { T-ratio }\end{array}$ & d.f. & P-value & \\
\hline \multicolumn{6}{|c|}{ For INTRCPT1, B0 } \\
\hline INTRCPT2, G00 & 0.447863 & 0.031295 & 14.311 & 41 & 0.000 \\
\hline \multicolumn{6}{|c|}{ For TIME slope, B1 } \\
\hline INTRCPT2, G10 & -0.003733 & 0.009953 & -0.375 & 163 & 0.70 \\
\hline \multicolumn{6}{|c|}{ For QUAD_TIM slope, B2 } \\
\hline INTRCPT'2, G20 & 0.012357 & 0.006568 & 1.881 & 163 & 0.062 \\
\hline
\end{tabular}

Standard error of $\operatorname{sigma}^{\wedge} 2=0.00263$

tau

INTRCPT1,B0 0.03338

Standard error of tau

INTRCPT1,B0 0.00835

Random level-1 coefficient Reliability estimate

INTRCPT1, G0 $\quad 0.874$

The value of the log-likelihood function at iteration $7=5.059164 \mathrm{E}+001$

The outcome variable is LG_CORT

Final estimation of fixed effects: 
For LG_SAA slope, B3

$\begin{array}{llllll}\text { INTRCPT2, G30 } & 0.066055 & 0.105757 & 0.625 & 163 & 0.533\end{array}$

For SAAXQ TI slope, B4

$\begin{array}{llllll}\text { INTRCPT2, G40 } & 0.010246 & 0.038970 & 0.263 & 163 & 0.793\end{array}$

Final estimation of variance components:

Random Effect Standard Variance df Chi-square P-value Deviation Component

INTRCPT1, u0 $0.18269 \quad 0.03338 \quad 41 \quad 333.54901 \quad 0.000$

level-1, $\quad \mathrm{r} \quad 0.15499 \quad 0.02402$

Statistics for the current model

Deviance $\quad=-101.183276$

Number of estimated parameters $=7$

Model comparison test

Chi-square statistic $=0.06866$

Degrees of freedom $=1$

P-value $\quad=\quad>.500$

\section{Model 6}

Level-1 Model

$$
\text { LG_CORT }=\mathrm{B} 0+\mathrm{B} 1 *(\mathrm{TIME})+\mathrm{B} 2 *\left(\mathrm{QUAD} \_\mathrm{TIM}\right)+\mathrm{B} 3 *\left(\mathrm{LG} \_\mathrm{SAA}\right)+\mathrm{B} 4 *(\mathrm{SAAXTIME})+\mathrm{r}
$$

Level-2 Model

$$
\begin{aligned}
& \mathrm{B} 0=\mathrm{G} 00+\mathrm{u} 0 \\
& \mathrm{~B} 1=\mathrm{G} 10 \\
& \mathrm{~B} 2=\mathrm{G} 20 \\
& \mathrm{~B} 3=\mathrm{G} 30 \\
& \mathrm{~B} 4=\mathrm{G} 40
\end{aligned}
$$

LG_SAA has been centered around the group mean.

$$
\begin{aligned}
& \text { Mixed Model } \\
& \text { LG_CORT }=\text { G00 } \\
& + \text { G10*TIME } \\
& + \text { G20*QUAD_TIM }
\end{aligned}
$$


+ G30*LG_SAA

+ G40*SA $\bar{A}$ XTIME

$+\mathrm{u} 0+\mathrm{r}$

$\operatorname{sigma}^{\wedge} 2=0.02401$

Standard error of $\operatorname{sigma}^{\wedge} 2=0.00263$

tau

INTRCPT1,B0 0.03292

Standard error of tau

INTRCPT1,B0 0.00826

Random level-1 coefficient Reliability estimate

INTRCPT1, G0 $\quad 0.872$

The value of the log-likelihood function at iteration $7=5.087204 \mathrm{E}+001$

The outcome variable is LG_CORT

Final estimation of fixed effects:

\begin{tabular}{|c|c|c|c|c|c|}
\hline Fixed Effect & $\begin{array}{c}\text { Standard } \\
\text { Coefficient Erro }\end{array}$ & $\begin{array}{r}\text { Approx. } \\
\text { or } \quad \text { T-ratio }\end{array}$ & d.f. & P-value & \\
\hline \multicolumn{6}{|c|}{ For INTRCPT1, B0 } \\
\hline \multicolumn{6}{|c|}{ For TIME slope, B1 } \\
\hline INTRCPT2, G1C & -0.003568 & 0.009952 & -0.358 & 163 & 0.720 \\
\hline \multicolumn{6}{|c|}{ For QUAD_TIM slope, B2 } \\
\hline INTRCPT2, G20 & 0.012747 & 0.006559 & 1.943 & 163 & 0.054 \\
\hline \multicolumn{6}{|c|}{ For LG_SAA slope, B3 } \\
\hline INTRCPT2, G3C & 0.021272 & 0.121117 & 0.176 & 163 & 0.861 \\
\hline \multicolumn{6}{|c|}{ For SAAXTIME slope, B4 } \\
\hline INTRCPT2, G4C & -0.042742 & 0.053627 & -0.797 & 163 & 0.427 \\
\hline
\end{tabular}

Final estimation of variance components:

Random Effect Standard Variance df Chi-square P-value Deviation Component

$\begin{array}{lllllll}\text { INTRCPT1, } & \text { u0 } & 0.18144 & 0.03292 & 41 & 329.66441 & 0.000\end{array}$ 
$\begin{array}{llll}\text { level-1, } & \mathrm{r} & 0.15496 & 0.02401\end{array}$

Statistics for the current model

Deviance $\quad=-101.744088$

Number of estimated parameters $=7$

Model comparison test

Chi-square statistic $=0.62948$

Degrees of freedom $=1$

$\mathrm{P}$-value $\quad=\quad>.500$

\section{Model 7}

Level-1 Model

LG_CORT $=\mathrm{B} 0+\mathrm{B} 1 *(\mathrm{TIME})+\mathrm{B} 2 *\left(\mathrm{QUAD} \_\mathrm{TIM}\right)+\mathrm{B} 3 *\left(\mathrm{LG}_{-} \mathrm{SAA}\right)+\mathrm{B} 4 *(\mathrm{SAAXTIME})+$ $\mathrm{B} 5 *(\mathrm{SAAX} \overline{\mathrm{X}} \mathrm{TI})+\mathrm{r}$

Level-2 Model

$$
\begin{aligned}
& \mathrm{B} 0=\mathrm{G} 00+\mathrm{u} 0 \\
& \mathrm{~B} 1=\mathrm{G} 10 \\
& \mathrm{~B} 2=\mathrm{G} 20 \\
& \mathrm{~B} 3=\mathrm{G} 30 \\
& \mathrm{~B} 4=\mathrm{G} 40 \\
& \mathrm{~B} 5=\mathrm{G} 50
\end{aligned}
$$

LG_SAA has been centered around the group mean.

$$
\begin{aligned}
& \text { Mixed Model } \\
& \text { LG_CORT }=\text { G00 } \\
& + \text { G10*TIME } \\
& + \text { G20*QUAD_TIM } \\
& \text { + G30*LG_SAA } \\
& \text { + G40*SAAXTIME } \\
& \text { + G50*SAAXQ_TI } \\
& \text { + u0+ r }
\end{aligned}
$$

$\operatorname{sigma}^{\wedge} 2=0.02399$

Standard error of $\operatorname{sigma}^{\wedge} 2=0.00263$

tau 
INTRCPT1,B0 0.03298

Standard error of tau

INTRCPT1,B0 0.00827

Random level-1 coefficient Reliability estimate

INTRCPT1, G0 $\quad 0.872$

The value of the log-likelihood function at iteration $7=5.092107 \mathrm{E}+001$

The outcome variable is LG_CORT

Final estimation of fixed effects:

\begin{tabular}{|c|c|c|c|c|c|}
\hline Fixed Effect & $\begin{array}{c}\text { Standard } \\
\text { Coefficient Erro }\end{array}$ & $\begin{array}{r}\text { Approx. } \\
\text { or } \quad \text { T-ratio }\end{array}$ & d.f. & P-value & \\
\hline \multicolumn{6}{|c|}{ For $\quad$ INTRCPT1, B0 } \\
\hline INTRCPT2, G00 & 0.448250 & 0.031144 & 14.393 & 41 & 0.000 \\
\hline \multicolumn{6}{|c|}{ For TIME slope, B1 } \\
\hline INTRCPT2, G10 & -0.003470 & 0.009952 & -0.349 & 162 & 0.728 \\
\hline \multicolumn{6}{|c|}{ For QUAD_TIM slope, B2 } \\
\hline INTRCPT2, G20 & 0.012606 & 0.006571 & 1.918 & 162 & 0.057 \\
\hline \multicolumn{6}{|c|}{ For LG_SAA slope, B3 } \\
\hline INTRCPT2, G30 & 0.010942 & 0.125522 & 0.087 & 162 & 0.931 \\
\hline \multicolumn{6}{|c|}{ For SAAXTIME slope, B4 } \\
\hline INTRCPT2, G40 & -0.056589 & 0.069586 & -0.813 & 162 & 0.417 \\
\hline \multicolumn{6}{|c|}{ For SAAXQ_TI slope, B5 } \\
\hline INTRCPT2, G50 & -0.015838 & 0.050548 & -0.313 & 162 & 0.75 \\
\hline
\end{tabular}

Final estimation of variance components:

Random Effect Standard Variance df Chi-square P-value Deviation Component

$\begin{array}{llllll}\text { INTRCPT1, u0 } & 0.18161 & 0.03298 & 41 & 330.48039 & 0.000\end{array}$
$\begin{array}{llll}\text { level-1, } & \text { r } & 0.15488 & 0.02399\end{array}$

Statistics for the current model

Deviance $\quad=-101.842139$ 
Number of estimated parameters $=8$

Model comparison test

Chi-square statistic $=0.09805$

Degrees of freedom $=1$

P-value $\quad=\quad>.500$

\section{Model 7.1}

Level-1 Model

LG_CORT $=$ B0 + B $1 *(T I M E)+B 2 *\left(Q U A D \_T I M\right)+B 3 *\left(L G \_S A A\right)+B 4 *($ SAAXTIME $)+$ $\mathrm{B} 5 *(\mathrm{SAAXQ} \mathrm{TI})+\mathrm{r}$

Level-2 Model

$$
\begin{aligned}
& \mathrm{B} 0=\mathrm{G} 00+\mathrm{u} 0 \\
& \mathrm{~B} 1=\mathrm{G} 10+\mathrm{u} 1 \\
& \mathrm{~B} 2=\mathrm{G} 20 \\
& \mathrm{~B} 3=\mathrm{G} 30 \\
& \mathrm{~B} 4=\mathrm{G} 40 \\
& \mathrm{~B} 5=\mathrm{G} 50
\end{aligned}
$$

LG_SAA has been centered around the group mean.

$$
\begin{aligned}
& \text { Mixed Model } \\
& \text { LG_CORT }=\text { G00 } \\
& \text { + G10*TIME } \\
& \text { + G20*QUAD_TIM } \\
& \text { + G30*LG_SAA } \\
& \text { + G40*SAAXTIME } \\
& \text { + G50*SAAXQ_TI } \\
& \text { + u0 + u1*TIME + r }
\end{aligned}
$$

$\operatorname{sigma}^{\wedge} 2=0.02128$

Standard error of $\operatorname{sigma}^{\wedge} 2=0.00269$

tau

$\begin{array}{lll}\text { INTRCPT1,B0 } & 0.02679 & 0.00284\end{array}$

$\begin{array}{lll}\text { TIME,B1 } & 0.00284 & 0.00109\end{array}$

Standard errors of tau

$\begin{array}{lll}\text { INTRCPT1,B0 } & 0.00730 & 0.00163\end{array}$

TIME,B1 $0.00163 \quad 0.00076$ 
tau (as correlations)

INTRCPT1,B0 $1.000 \quad 0.526$

TIME,B1 0.5261 .000

Random level-1 coefficient Reliability estimate

$\begin{array}{lc}\text { INTRCPT1, G0 } & 0.804 \\ \text { TIME, G1 } & 0.335\end{array}$

The value of the log-likelihood function at iteration $18=5.512945 \mathrm{E}+001$

The outcome variable is LG_CORT

Final estimation of fixed effects:

\begin{tabular}{|c|c|c|c|c|c|}
\hline Fixed Effect & $\begin{array}{c}\text { Standard } \\
\text { Coefficient Erro }\end{array}$ & $\begin{array}{r}\text { Approx. } \\
\text { T-ratio }\end{array}$ & d.f. & P-value & \\
\hline \multicolumn{6}{|c|}{ For INTRCPT1, B0 } \\
\hline \multicolumn{6}{|c|}{ For TIME slope, B1 } \\
\hline INTRCPT2, G1 & -0.003913 & 0.010671 & -0.367 & 41 & 0.716 \\
\hline \multicolumn{6}{|c|}{ For QUAD_TIM slope, B2 } \\
\hline $\begin{array}{l}\text { INTRCPT } 2, \text { G } 2 \\
\text { For LG SAA sl }\end{array}$ & ee. B3 0.012734 & 0.006 & 054 & 121 & 0.042 \\
\hline INTRCPT2, G3 & -0.019857 & 0.124469 & -0.160 & 121 & 0.874 \\
\hline \multicolumn{6}{|c|}{ For SAAXTIME slope, B4 } \\
\hline INTRCPT2, G4 & -0.051997 & 0.072871 & -0.714 & 121 & 0.477 \\
\hline \multicolumn{6}{|c|}{ For SAAXQ_TI slope, B5 } \\
\hline INTRCPT2, G5 & -0.013151 & 0.048713 & -0.270 & 121 & 0.788 \\
\hline
\end{tabular}

Final estimation of variance components:

Random Effect Standard Variance df Chi-square P-value Deviation Component

\begin{tabular}{ccccccc}
\hline INTRCPT1, u0 & 0.16366 & 0.02679 & 41 & 218.48118 & 0.000 \\
TIME slope, u1 & 0.03295 & 0.00109 & 41 & 63.83974 & 0.013 \\
level-1, r & 0.14587 & 0.02128 & & &
\end{tabular}

Statistics for the current model 
Deviance $\quad=-110.258891$

Number of estimated parameters $=10$

Model comparison test

Chi-square statistic $=8.41675$

Degrees of freedom $=2$

P-value $\quad=0.015$

\section{Model 7.2}

Level-1 Model

LG_CORT $=$ B $0+$ B $1 *($ TIME $)+$ B2 $*($ QUAD_TIM $)+$ B $3 *\left(L G \_S A A\right)+B 4 *($ SAAXTIME $)+$ $\mathrm{B} 5 *\left(\mathrm{SAAXQ}{ }_{-} \mathrm{TI}\right)+\mathrm{r}$

Level-2 Model

$$
\begin{aligned}
& \mathrm{B} 0=\mathrm{G} 00+\mathrm{u} 0 \\
& \mathrm{~B} 1=\mathrm{G} 10 \\
& \mathrm{~B} 2=\mathrm{G} 20+\mathrm{u} 2 \\
& \mathrm{~B} 3=\mathrm{G} 30 \\
& \mathrm{~B} 4=\mathrm{G} 40 \\
& \mathrm{~B} 5=\mathrm{G} 50
\end{aligned}
$$

LG_SAA has been centered around the group mean.

$$
\begin{aligned}
& \text { Mixed Model } \\
& \text { LG_CORT = G00 } \\
& \text { + G10*TIME } \\
& \text { + G20*QUAD_TIM } \\
& \text { + G30*LG_SAA } \\
& \text { + G40*SAAXTIME } \\
& \text { + G50*SAAXQ_TI } \\
& \text { + u0 + u2*QUAD_TIM + r }
\end{aligned}
$$

$\operatorname{sigma}^{\wedge} 2=0.01932$

Standard error of sigma ${ }^{\wedge} 2=0.00244$

tau

$$
\begin{array}{lll}
\text { INTRCPT1,B0 } & 0.03054 & -0.00250 \\
\text { QUAD_TIM,B2 } & -0.00250 & 0.00081
\end{array}
$$

Standard errors of tau

$\begin{array}{lll}\text { INTRCPT1,B0 } & 0.00761 & 0.00121\end{array}$

$\begin{array}{lll}\text { QUAD_TIM,B2 } & 0.00121 & 0.00038\end{array}$ 
tau (as correlations)

INTRCPT1,B0 $1.000-0.502$

QUAD_TIM,B2 -0.502 1.000

Random level-1 coefficient Reliability estimate

INTRCPT1, G0 $\quad 0.874$

QUAD_TIM, G2 $\quad 0.485$

The value of the log-likelihood function at iteration $13=5.810132 \mathrm{E}+001$ 
The outcome variable is LG_CORT

Final estimation of fixed effects:

\begin{tabular}{|c|c|c|c|c|c|}
\hline Fixed Effect & $\begin{array}{c}\text { Standard } \\
\text { Coefficient Erro }\end{array}$ & $\begin{array}{rr} & \text { Approx. } \\
\text { or } & \text { T-ratio }\end{array}$ & d.f. & P-value & \\
\hline \multicolumn{6}{|c|}{ For INTRCPT1, B0 } \\
\hline INTRCPT2, G00 & 0.448835 & 0.029635 & 15.145 & 41 & 0.000 \\
\hline \multicolumn{6}{|c|}{ For TIME slope, B1 } \\
\hline INTRCPT2, G10 & -0.004024 & 0.008943 & -0.450 & 121 & 0.654 \\
\hline \multicolumn{6}{|c|}{ For QUAD TIM slope, B2 } \\
\hline INTRCPT2, G20 & 0.012700 & 0.007379 & 1.721 & 41 & 0.093 \\
\hline \multicolumn{6}{|c|}{ For LG_SAA slope, B3 } \\
\hline INTRCPT2, G30 & -0.044759 & 0.122530 & -0.365 & 121 & 0.716 \\
\hline \multicolumn{6}{|c|}{ For SAAXTIME slope, B4 } \\
\hline INTRCPT2, G40 & -0.062670 & 0.063017 & -0.994 & 121 & 0.322 \\
\hline \multicolumn{6}{|c|}{ For SAAXQ TI slope, B5 } \\
\hline INTRCPT2, G50 & -0.022977 & 0.049630 & -0.463 & 121 & 0.644 \\
\hline
\end{tabular}

Final estimation of variance components:

Random Effect Standard Variance df Chi-square P-value Deviation Component

$\begin{array}{lllllll} & \text { INTRCPT1, u0 } & 0.17476 & 0.03054 & 41 & 348.15708 & 0.000 \\ \text { QUAD_TIM slope, u2 } & 0.02848 & 0.00081 & 41 & 82.19075 & 0.000 \\ \text { level-1, } \quad \mathrm{r} & 0.13901 & 0.01932 & & & & \end{array}$

Statistics for the current model

Deviance $\quad=-116.202640$

Number of estimated parameters $=10$

Model comparison test

Chi-square statistic $=14.36050$
Degrees of freedom $=2$
P-value $=0.001$

\section{Model 7.3}

Level-1 Model 
LG_CORT $=$ B0 + B1*(TIME $)+B 2 *\left(Q U A D \_T I M\right)+B 3 *\left(L G \_S A A\right)+B 4 *(S A A X T I M E)+$ $\mathrm{B} 5 *\left(\mathrm{SAAX} \mathrm{Q}_{-} \mathrm{TI}\right)+\mathrm{r}$

Level-2 Model

$$
\begin{aligned}
& \mathrm{B} 0=\mathrm{G} 00+\mathrm{u} 0 \\
& \mathrm{~B} 1=\mathrm{G} 10 \\
& \mathrm{~B} 2=\mathrm{G} 20 \\
& \mathrm{~B} 3=\mathrm{G} 30+\mathrm{u} 3 \\
& \mathrm{~B} 4=\mathrm{G} 40 \\
& \mathrm{~B} 5=\mathrm{G} 50
\end{aligned}
$$

LG_SAA has been centered around the group mean.

$$
\begin{aligned}
& \text { Mixed Model } \\
& \text { LG_CORT }=\text { G00 } \\
& \text { + G10*TIME } \\
& \text { + G20*QUAD_TIM } \\
& \text { + G30*LG_SAA } \\
& \text { + G40*SAAXTIME } \\
& \text { + G50*SAAXQ_TI } \\
& \text { + u0 + u3*LG_SAA + r }
\end{aligned}
$$

$\operatorname{sigma}^{\wedge} 2=0.02142$

Standard error of $\operatorname{sigma}^{\wedge} 2=0.00257$

$$
\begin{array}{lcc}
\text { tau } & & \\
\text { INTRCPT1,B0 } & 0.03410 & 0.01807 \\
\text { LG_SAA,B3 } & 0.01807 & 0.18271
\end{array}
$$

Standard errors of tau

$$
\begin{array}{ccc}
\text { INTRCPT1,B0 } & 0.00840 & 0.02546 \\
\text { LG_SAA,B3 } & 0.02546 & 0.12942
\end{array}
$$

tau (as correlations)

INTRCPT1,B0 $1.000 \quad 0.229$

LG_SAA,B3 0.2291 .000

Random level-1 coefficient Reliability estimate

$\begin{array}{cc}\text { INTRCPT1, G0 } & 0.888 \\ \text { LG_SAA, G3 } & 0.259\end{array}$

The value of the log-likelihood function at iteration $13=5.325547 \mathrm{E}+001$ 
The outcome variable is LG_CORT

Final estimation of fixed effects:

\begin{tabular}{|c|c|c|c|c|c|}
\hline Fixed Effect & $\begin{array}{c}\text { Standard } \\
\text { Coefficient Err }\end{array}$ & $\begin{array}{l}\text { Approx } \\
\text { T-ratic }\end{array}$ & d.f. & P-value & \\
\hline \multicolumn{6}{|c|}{ For INTRCPT1, B0 } \\
\hline INTRCPT2, G00 & 0.447252 & 0.031322 & 14.279 & 41 & 0.000 \\
\hline \multicolumn{6}{|c|}{ For TIME slope, B1 } \\
\hline INTRCPT2, G10 & -0.002080 & 0.009676 & -0.215 & 121 & 0.830 \\
\hline \multicolumn{6}{|c|}{ For QUAD_TIM slope, B2 } \\
\hline INTRCPT2, G20 & 0.014770 & 0.006349 & 2.326 & 121 & 0.022 \\
\hline \multicolumn{6}{|c|}{ For LG_SAA slope, B3 } \\
\hline INTRCPPT2, G30 & 0.088260 & 0.145581 & 0.606 & 41 & 0.548 \\
\hline \multicolumn{6}{|c|}{ For SAAXTIME slope, B4 } \\
\hline INTRCPT2, G40 & -0.017040 & 0.068470 & -0.249 & 121 & 0.804 \\
\hline \multicolumn{6}{|c|}{ For SAAXQ_TI slope, B5 } \\
\hline INTRCPT2, G50 & -0.017504 & 0.049914 & -0.351 & 121 & 0.726 \\
\hline
\end{tabular}

Final estimation of variance components:

Random Effect Standard Variance df Chi-square P-value Deviation Component

$\begin{array}{llccccc}\text { INTRCPT1, u0 } & 0.18466 & 0.03410 & 41 & 376.10762 & 0.000 \\ \text { LG_SAA slope, } \mathrm{u} 3 & 0.42744 & 0.18271 & 41 & 50.97844 & 0.137 \\ \text { level-1, } & \mathrm{r} & 0.14635 & 0.02142 & & & \end{array}$

Statistics for the current model

Deviance $\quad=-106.510940$

Number of estimated parameters $=10$

Model comparison test

Chi-square statistic $=4.66880$

Degrees of freedom $=2$

$\mathrm{P}$-value $\quad 0.095$

\section{Model 7.4}

Level-1 Model 
LG_CORT $=$ B0 + B $1 *(T I M E)+B 2 *\left(Q U A D \_T I M\right)+B 3 *\left(L G \_S A A\right)+B 4 *(S A A X T I M E)+$ $\mathrm{B} 5 *(\mathrm{SAAXQ} \mathrm{TI})+\mathrm{r}$

Level-2 Model

$$
\begin{aligned}
& \mathrm{B} 0=\mathrm{G} 00+\mathrm{u} 0 \\
& \mathrm{~B} 1=\mathrm{G} 10+\mathrm{u} 1 \\
& \mathrm{~B} 2=\mathrm{G} 20+\mathrm{u} 2 \\
& \mathrm{~B} 3=\mathrm{G} 30 \\
& \mathrm{~B} 4=\mathrm{G} 40 \\
& \mathrm{~B} 5=\mathrm{G} 50
\end{aligned}
$$

LG_SAA has been centered around the group mean.

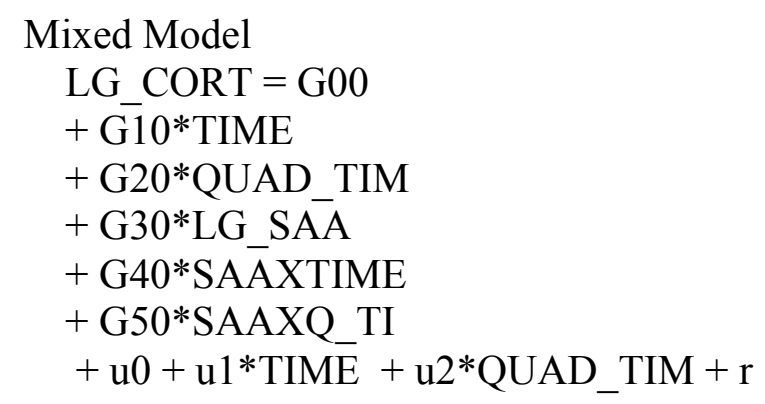

$\operatorname{sigma}^{\wedge} 2=0.01858$

Standard error of $\operatorname{sigma}^{\wedge} 2=\quad 0.00287$

tau

INTRCPT1,B0 $0.02869 \quad 0.00119 \quad-0.00151$

$\begin{array}{llll}\text { TIME,B } 1 & 0.00119 & 0.00030 & -0.00038\end{array}$

$\begin{array}{llll}\text { QUAD_TIM,B2 } & -0.00151 & -0.00038 & 0.00050\end{array}$

Standard errors of tau

$\begin{array}{llll}\text { INTRCPT1,B0 } & 0.00759 & 0.00174 & 0.00128\end{array}$

$\begin{array}{llll}\text { TIME,B } 1 & 0.00174 & 0.00090 & 0.00047\end{array}$

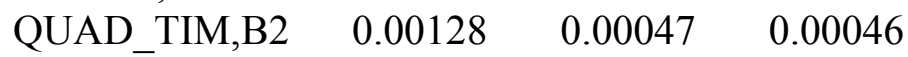

tau (as correlations)

INTRCPT1,B0 $1.000 \quad 0.408-0.399$

TIME,B1 $0.408 \quad 1.000-0.990$

QUAD_TIM,B2 -0.399-0.990 1.000

Random level-1 coefficient Reliability estimate 
INTRCPT1, G0

TIME, G1

QUAD_TIM, G2
0.826

0.086

0.264

The value of the log-likelihood function at iteration $5844=5.928108 \mathrm{E}+001$

The outcome variable is LG_CORT

Final estimation of fixed effects:

\begin{tabular}{|c|c|c|c|c|c|}
\hline Fixed Effect & $\begin{array}{c}\text { Standard } \\
\text { Coefficient Error }\end{array}$ & Approx. & d.f. & \multicolumn{2}{|l|}{ P-value } \\
\hline \multicolumn{6}{|c|}{ For INTRCPT1, B0 } \\
\hline INTRCPT2, G00 & 0.449907 & 0.028798 & 15.623 & 41 & 0.000 \\
\hline \multicolumn{6}{|c|}{ For TIME slope, B1 } \\
\hline INTRCPT2, G10 & -0.004279 & 0.009169 & -0.467 & 41 & 0.643 \\
\hline \multicolumn{6}{|c|}{ For QUAD_TIM slope, B2 } \\
\hline INTRCPT2, G20 & 0.013109 & 0.006770 & 1.936 & 41 & 0.060 \\
\hline \multicolumn{6}{|c|}{ For LG_SAA slope, B3 } \\
\hline INTRCPT2, G30 & -0.058451 & 0.124465 & -0.470 & 80 & 0.640 \\
\hline \multicolumn{6}{|c|}{ For SAAXTIME slope, B4 } \\
\hline INTRCPT2, G40 & -0.058670 & 0.063753 & -0.920 & 80 & 0.360 \\
\hline \multicolumn{6}{|c|}{ For SAAXQ_TI slope, B5 } \\
\hline INTRCPT2, G50 & -0.007257 & 0.048452 & -0.150 & 80 & 0.881 \\
\hline
\end{tabular}

The outcome variable is LG_CORT

Final estimation of variance components:

Random Effect Standard Variance df Chi-square P-value Deviation Component

$\begin{array}{lllllll}\text { INTRCPT1, u0 } & 0.16939 & 0.02869 & 41 & 250.16030 & 0.000 \\ \text { TIME slope, u1 } & 0.01725 & 0.00030 & 41 & 40.37455 & >.500 \\ \text { QUAD_TIM slope, u2 } & 0.02237 & 0.00050 & 41 & 52.37501 & 0.110 \\ \text { level-1, r } & 0.13631 & 0.01858 & & & & \end{array}$

Statistics for the current model

Deviance $\quad=-118.562156$

Number of estimated parameters $=13$

Model comparison test 
Chi-square statistic $=16.72002$

Degrees of freedom $=5$

P-value $\quad=0.005$

\section{Model 7.5}

Level-1 Model

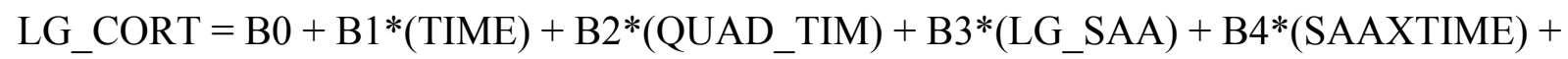
$\mathrm{B} 5 *(\mathrm{SAAX} \overline{\mathrm{X}} \mathrm{TI})+\mathrm{r}$

Level-2 Model

$$
\begin{aligned}
& \mathrm{B} 0=\mathrm{G} 00+\mathrm{u} 0 \\
& \mathrm{~B} 1=\mathrm{G} 10 \\
& \mathrm{~B} 2=\mathrm{G} 20+\mathrm{u} 2 \\
& \mathrm{~B} 3=\mathrm{G} 30+\mathrm{u} 3 \\
& \mathrm{~B} 4=\mathrm{G} 40 \\
& \mathrm{~B} 5=\mathrm{G} 50
\end{aligned}
$$

LG_SAA has been centered around the group mean.

$$
\begin{aligned}
& \text { Mixed Model } \\
& \text { LG_CORT }=\text { G00 } \\
& + \text { G10*TIME } \\
& + \text { G20*QUAD_TIM } \\
& + \text { G30*LG_SAA } \\
& \text { + G40*SAAXTIME } \\
& \text { + G50*SAAXQ_TI } \\
& \text { + } \mathrm{u} 0+\mathrm{u} 2 * \text { QUAD_TIM + u3*LG_SAA + r }
\end{aligned}
$$

$\operatorname{sigma}^{\wedge} 2=0.01306$

Standard error of sigma^ $2=0.00184$

$\begin{array}{llll}\text { tau } & & & \\ \text { INTRCPT1,B0 } & 0.03249 & -0.00238 & 0.01591 \\ \text { QUAD_TIM,B2 } & -0.00238 & 0.00136 & -0.01654 \\ \text { LG_SAA,B3 } & 0.01591 & -0.01654 & 0.35653\end{array}$

Standard errors of tau

$\begin{array}{cccc}\text { INTRCPT1,B0 } & 0.00774 & 0.00135 & 0.02550 \\ \text { QUAD_TIM,B2 } & 0.00135 & 0.00046 & 0.00677 \\ \text { LG_SAA,B3 } & 0.02550 & 0.00677 & 0.15611\end{array}$

tau (as correlations) 
INTRCPT1,B0 $1.000-0.358 \quad 0.148$

QUAD_TIM,B2 -0.358 $1.000-0.751$

LG_SAA,B3 $0.148-0.751 \quad 1.000$

Random level-1 coefficient Reliability estimate

\begin{tabular}{lc}
\hline INTRCPT1, G0 & 0.906 \\
QUAD_TIM, G2 & 0.611 \\
LG_SAA, G3 & 0.390
\end{tabular}

The value of the log-likelihood function at iteration $23=7.056640 \mathrm{E}+001$

The outcome variable is LG_CORT

Final estimation of fixed effects:

\begin{tabular}{|c|c|c|c|c|c|}
\hline Fixed Effect & $\begin{array}{c}\text { Standard } \\
\text { Coefficient Errol }\end{array}$ & $\begin{array}{r}\text { Approx. } \\
\text { T-ratio }\end{array}$ & d.f. & P-value & \\
\hline \multicolumn{6}{|c|}{ For INTRCPT1, B0 } \\
\hline \multicolumn{6}{|c|}{ For TIME slope, B1 } \\
\hline INTRCPT2, G10 & -0.002475 & 0.007755 & -0.319 & 80 & 0.750 \\
\hline \multicolumn{6}{|c|}{ For QUAD_TIM slope, B2 } \\
\hline INTRCPT2, G20 & 0.016188 & 0.007682 & 2.107 & 41 & 0.041 \\
\hline \multicolumn{6}{|c|}{ For LG SAA slope, B3 } \\
\hline INTRCPT2, G30 & 0.069013 & 0.146916 & 0.470 & 41 & 0.641 \\
\hline \multicolumn{6}{|c|}{ For SAAXTIME slope, B4 } \\
\hline INTRCPT2, G40 & 0.020238 & 0.055943 & 0.362 & 80 & 0.718 \\
\hline \multicolumn{6}{|c|}{ For SAAXQ TI slope, B5 } \\
\hline INTRCPT2, G50 & 0.006011 & 0.045773 & 0.131 & 80 & 0.896 \\
\hline
\end{tabular}

Final estimation of variance components:

Random Effect Standard Variance df Chi-square P-value Deviation Component

\begin{tabular}{ccccccc}
\hline INTRCPT1, u0 & 0.18024 & 0.03249 & 41 & 501.84897 & 0.000 \\
QUAD_TIM slope, u2 & 0.03688 & 0.00136 & 41 & 134.85225 & 0.000 \\
LG_SAA slope, u3 & 0.59710 & 0.35653 & 41 & 99.59270 & 0.000 \\
level-1, r r & 0.11429 & 0.01306 & & &
\end{tabular}


Statistics for the current model

Deviance $\quad=-141.132809$

Number of estimated parameters $=13$

Model comparison test

Chi-square statistic $=39.29067$

Degrees of freedom $=5$

P-value $\quad=\quad<0.001$

\section{Model 8}

Level-1 Model

LG_CORT $=$ B0 + B1 $*($ TIME $)+$ B2 $*($ QUAD_TIM $)+$ B3 $*\left(L G \_S A A\right)+B 4 *($ SAAXTIME $)+$ $\mathrm{B} 5 *(\mathrm{SAAXQ} \mathrm{TI})+\mathrm{r}$

Level-2 Model

$$
\begin{aligned}
& \mathrm{B} 0=\mathrm{G} 00+\mathrm{u} 0 \\
& \mathrm{~B} 1=\mathrm{G} 10 \\
& \mathrm{~B} 2=\mathrm{G} 20+\mathrm{u} 2 \\
& \mathrm{~B} 3=\mathrm{G} 30 \\
& \mathrm{~B} 4=\mathrm{G} 40 \\
& \mathrm{~B} 5=\mathrm{G} 50
\end{aligned}
$$

LG_SAA has been centered around the group mean.

$$
\begin{aligned}
& \text { Mixed Model } \\
& \text { LG_CORT = G00 } \\
& \text { + G10*TIME } \\
& \text { + G20*QUAD_TIM } \\
& \text { + G30*LG_SAA } \\
& \text { + G40*SAAXTIME } \\
& \text { + G50*SAAXQ_TI } \\
& \text { + u0 + u2*QUAD_TIM + r }
\end{aligned}
$$

$\operatorname{sigma}^{\wedge} 2=0.01932$

Standard error of $\operatorname{sigma}^{\wedge} 2=0.00244$

tau

INTRCPT1,B0 $0.03054 \quad-0.00250$

QUAD_TIM,B2 $\quad-0.00250 \quad 0.00081$

Standard errors of tau 
$\begin{array}{lll}\text { INTRCPT1,B0 } & 0.00761 \quad 0.00121\end{array}$

QUAD_TIM,B2 $0.00121 \quad 0.00038$

tau (as correlations)

INTRCPT1,B0 $1.000-0.502$

QUAD_TIM,B2 -0.502 1.000

Random level-1 coefficient Reliability estimate

$\begin{array}{lc}\text { INTRCPT1, G0 } & 0.874 \\ \text { QUAD_TIM, G2 } & 0.485 \\ -\end{array}$

The value of the log-likelihood function at iteration $13=5.810132 \mathrm{E}+001$

The outcome variable is LG_CORT

Final estimation of fixed effects:

\begin{tabular}{|c|c|c|c|c|c|}
\hline Fixed Effect & $\begin{array}{c}\text { Standard } \\
\text { Coefficient Erro }\end{array}$ & $\begin{array}{r}\text { Approx. } \\
\text { or } \quad \text { T-ratio }\end{array}$ & d.f. & P-value & \\
\hline \multicolumn{5}{|c|}{ For INTRCPT1, B0 } & 0.000 \\
\hline \multicolumn{6}{|c|}{ For TIME slope, B1 } \\
\hline INTRCPT2, G10 & -0.004024 & 0.008943 & -0.450 & 121 & 0.654 \\
\hline \multicolumn{6}{|c|}{ For QUAD_TIM slope, B2 } \\
\hline INTRCPT2, G20 & 0.012700 & 0.007379 & 1.721 & 41 & 0.093 \\
\hline \multicolumn{6}{|c|}{ For LG_SAA slope, B3 } \\
\hline INTRCPT2, G30 & -0.044759 & 0.122530 & -0.365 & 121 & 0.716 \\
\hline \multicolumn{6}{|c|}{ For SAAXTIME slope, B4 } \\
\hline INTRCPT2, G40 & -0.062670 & 0.063017 & -0.994 & 121 & 0.322 \\
\hline \multicolumn{6}{|c|}{ For SAAXQ TI slope, B5 } \\
\hline INTRCPT2, G50 & -0.022977 & 0.049630 & -0.463 & 121 & 0.644 \\
\hline
\end{tabular}

Final estimation of variance components:

Random Effect Standard Variance df Chi-square P-value Deviation Component

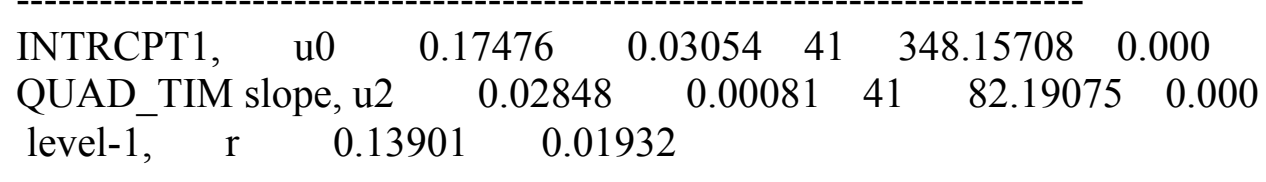


Statistics for the current model

Deviance $\quad=-116.202640$

Number of estimated parameters $=10$

Model comparison test

Chi-square statistic $=14.36050$

Degrees of freedom $=2$

P-value $\quad=0.001$

\section{Model 9}

Level-1 Model

LG_CORT $=\mathrm{B} 0+\mathrm{B} 1 *(\mathrm{TIME})+\mathrm{B} 2 *\left(\mathrm{QUAD} \_\mathrm{TIM}\right)+\mathrm{B} 3 *\left(\mathrm{LG} \_\mathrm{SAA}\right)+\mathrm{B} 4 *(\mathrm{SAAXTIME})+$ $\mathrm{B} 5 *\left(\mathrm{SAAX}{ }^{2} \mathrm{TI}\right)+\mathrm{r}$

Level-2 Model

$$
\begin{aligned}
& \mathrm{B} 0=\mathrm{G} 00+\mathrm{G} 01 *(\mathrm{CDI} \text { TOTA })+\mathrm{u} 0 \\
& \mathrm{~B} 1=\mathrm{G} 10 \\
& \mathrm{~B} 2=\mathrm{G} 20+\mathrm{u} 2 \\
& \mathrm{~B} 3=\mathrm{G} 30 \\
& \mathrm{~B} 4=\mathrm{G} 40 \\
& \mathrm{~B} 5=\mathrm{G} 50
\end{aligned}
$$

LG_SAA has been centered around the group mean.

CDI_TOTA has been centered around the grand mean.

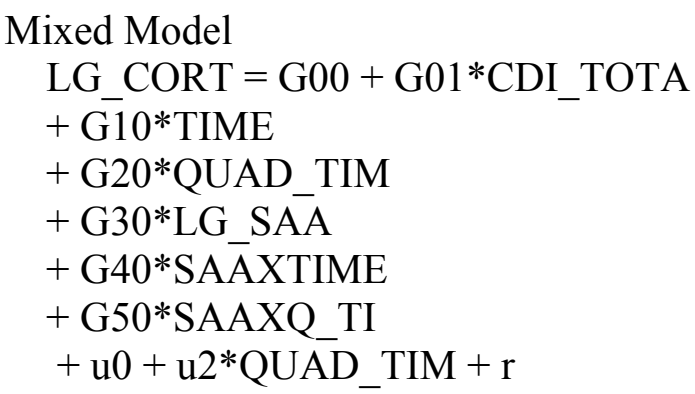

$\operatorname{sigma}^{\wedge} 2=0.01932$

Standard error of $\operatorname{sigma}^{\wedge} 2=0.00244$

tau 


$$
\begin{array}{llc}
\text { INTRCPT1,B0 } & 0.03036 & -0.00249 \\
\text { QUAD_TIM,B2 } & -0.00249 & 0.00081
\end{array}
$$

Standard errors of tau

$\begin{array}{lcc}\text { INTRCPT1,B0 } & 0.00757 & 0.00121 \\ \text { QUAD_TIM,B2 } & 0.00121 & 0.00038\end{array}$

tau (as correlations)

INTRCPT1,B0 $1.000-0.500$

QUAD_TIM,B2 -0.5001 .000$

Random level-1 coefficient Reliability estimate

\begin{tabular}{lc} 
INTRCPT1, G0 & 0.873 \\
QUAD_TIM, G2 & 0.486 \\
\hline.-----
\end{tabular}

The value of the log-likelihood function at iteration $13=5.819549 \mathrm{E}+001$

The outcome variable is LG_CORT

\begin{tabular}{|c|c|c|c|c|c|}
\hline Fixed Effect & $\begin{array}{c}\text { Standard } \\
\text { Coefficient Erro }\end{array}$ & $\begin{array}{r}\text { Approx. } \\
\text { or } \quad \text { T-ratio }\end{array}$ & d.f. & P-value & \\
\hline \multicolumn{6}{|c|}{ For INTRCPT1, B0 } \\
\hline INTRCPT2, G00 & 0.449382 & 0.029587 & 15.189 & 40 & 0.000 \\
\hline CDI TOTA, G01 & 0.002028 & 0.004666 & 0.435 & 40 & 0.666 \\
\hline \multicolumn{6}{|c|}{ For $\quad$ TIME slope, B1 } \\
\hline INTRCPT2, G10 & -0.004048 & 0.008941 & -0.453 & 121 & 0.652 \\
\hline \multicolumn{6}{|c|}{ For QUAD TIM slope, B2 } \\
\hline INTRCPT̄2, G20 & 0.012748 & 0.007383 & 1.727 & 41 & 0.092 \\
\hline \multicolumn{6}{|c|}{ For LG_SAA slope, B3 } \\
\hline INTRCPT2, G30 & -0.049249 & 0.122925 & -0.401 & 121 & 0.689 \\
\hline \multicolumn{6}{|c|}{ For SAAXTIME slope, B4 } \\
\hline INTRCPT2, G40 & -0.064493 & 0.063127 & -1.022 & 121 & 0.309 \\
\hline \multicolumn{6}{|c|}{ For SAAXQ_TI slope, B5 } \\
\hline INTRCPT2, G50 & -0.022479 & 0.049647 & -0.453 & 121 & 0.652 \\
\hline
\end{tabular}

Final estimation of fixed effects:

Final estimation of variance components:

Random Effect Standard Variance df Chi-square P-value Deviation Component 
$\begin{array}{lllllll}\text { INTRCPT1, u0 } & 0.17423 & 0.03036 & 40 & 346.41998 & 0.000\end{array}$ QUAD_TIM slope, u2 $\quad 0.02853 \quad 0.00081 \quad 41 \quad 82.32576 \quad 0.000$ level-1, $\quad \mathrm{r} \quad 0.13899 \quad 0.01932$

Statistics for the current model

Deviance $\quad=-116.390985$

Number of estimated parameters $=11$

Model comparison test

$\begin{array}{lcc}\text { Chi-square statistic } & =0.18835 \\ \text { Degrees of freedom } & =1 \\ \text { P-value }= & >.500\end{array}$

\section{Model 10}

Level-1 Model

LG_CORT $=$ B0 + B1 $*($ TIME $)+$ B2 $*($ QUAD_TIM $)+$ B3 $*\left(L G \_S A A\right)+B 4 *($ SAAXTIME $)+$ $\mathrm{B} 5 *(\mathrm{SAAXQ} \mathrm{TI})+\mathrm{r}$

Level-2 Model

$$
\begin{aligned}
& \mathrm{B} 0=\mathrm{G} 00+\mathrm{G} 01 *(\mathrm{CDI} \text { TOTA })+\mathrm{u} 0 \\
& \mathrm{~B} 1=\mathrm{G} 10+\mathrm{G} 11 *(\mathrm{CDI} \text { IOTA }) \\
& \mathrm{B} 2=\mathrm{G} 20+\mathrm{G} 21 *(\mathrm{CDI} \text {-TOTA })+\mathrm{u} 2 \\
& \mathrm{~B} 3=\mathrm{G} 30 \\
& \mathrm{~B} 4=\mathrm{G} 40 \\
& \mathrm{~B} 5=\mathrm{G} 50
\end{aligned}
$$

LG_SAA has been centered around the group mean.

CDI_TOTA has been centered around the grand mean.

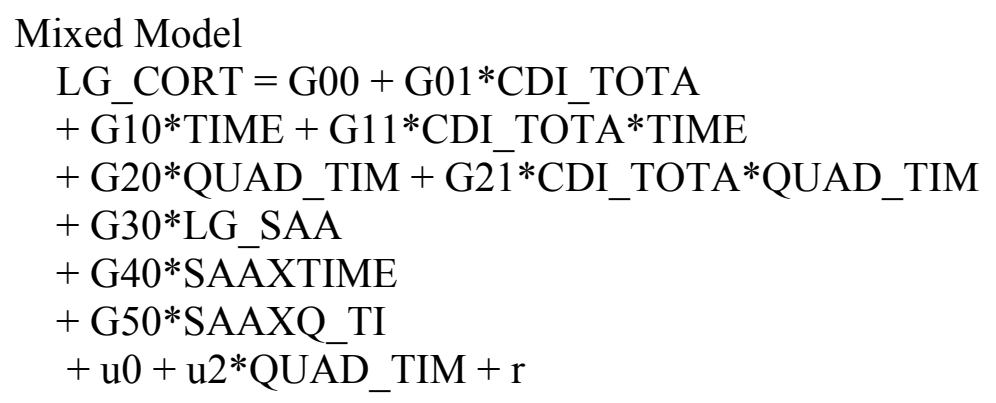

sigma $^{\wedge} 2=0.01914$ 
Standard error of sigma^2 ${ }^{\wedge} \quad 0.00242$

tau

INTRCPT1,B0 $0.03046 \quad-0.00248$

QUAD_TIM,B2 $\quad-0.00248 \quad 0.00082$

Standard errors of tau

$\begin{array}{lcc}\text { INTRCPT1,B0 } & 0.00758 & 0.00121 \\ \text { QUAD_TIM,B2 } & 0.00121 & 0.00038\end{array}$

tau (as correlations)

INTRCPT1,B0 $1.000-0.495$

QUAD_TIM,B2 -0.495 1.000

Random level-1 coefficient Reliability estimate

$\begin{array}{lc}\text { INTRCPT1, G0 } & 0.875 \\ \text { QUAD_TIM, G2 } & 0.492\end{array}$

The value of the log-likelihood function at iteration $13=5.868709 \mathrm{E}+001$

The outcome variable is LG_CORT

Final estimation of fixed effects:

\begin{tabular}{|c|c|c|c|c|c|}
\hline Fixed Effect & $\begin{array}{c}\text { Standard } \\
\text { Coefficient Erro }\end{array}$ & $\begin{array}{r}\text { Approx. } \\
\text { or } \quad \text { T-ratio }\end{array}$ & d.f. & P-value & \\
\hline \multicolumn{6}{|c|}{ For INTRCPT1, B0 } \\
\hline INTRCPT2, G00 & 0.449743 & 0.029607 & 15.190 & 40 & 0.000 \\
\hline CDI_TOTA, G0 & 0.003188 & 0.004949 & 0.644 & 40 & 0.523 \\
\hline \multicolumn{6}{|c|}{ For TIME slope, B1 } \\
\hline INTRCPT2, G10 & -0.004526 & 0.008914 & -0.508 & 120 & 0.613 \\
\hline CDI_TOTA, G1 & -0.001498 & 0.001510 & -0.992 & 120 & 0.323 \\
\hline \multicolumn{6}{|c|}{ For QŪAD_TIM slope, B2 } \\
\hline INTRCPT2, G20 & 0.012607 & 0.007386 & 1.707 & 40 & 0.096 \\
\hline CDI_TOTA, G2 & -0.000696 & 0.001232 & -0.564 & 40 & 0.576 \\
\hline \multicolumn{6}{|c|}{ For LG_SAA slope, B3 } \\
\hline INTRCPT2, G30 & -0.047841 & 0.123267 & -0.388 & 120 & 0.699 \\
\hline \multicolumn{6}{|c|}{ For SAAXTIME slope, B4 } \\
\hline INTRCPT2, G40 & -0.053158 & 0.063881 & -0.832 & 120 & 0.407 \\
\hline \multicolumn{6}{|c|}{ For SAAXQ_TI slope, B5 } \\
\hline INTRCPT2, G50 & -0.016036 & 0.049908 & -0.321 & 120 & 0.749 \\
\hline
\end{tabular}


Final estimation of variance components:

Random Effect Standard Variance df Chi-square P-value
Deviation Component

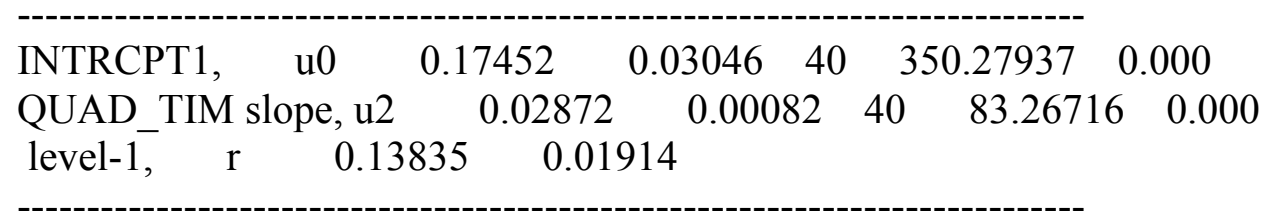

Statistics for the current model

Deviance $\quad=-117.374177$

Number of estimated parameters $=13$

Model comparison test

Chi-square statistic $=0.98319$
Degrees of freedom $=2$
P-value $=>.500$

\section{Model 11}

Level-1 Model

LG_CORT $=$ B0 + B1 $*($ TIME $)+$ B2 $*\left(Q U A D \_T I M\right)+B 3 *\left(L G \_S A A\right)+B 4 *($ SAAXTIME $)+$ $\mathrm{B} 5 *(\mathrm{SAAX} \overline{\mathrm{Q}} \mathrm{TI})+\mathrm{r}$

Level-2 Model

$$
\begin{aligned}
& \mathrm{B} 0=\mathrm{G} 00+\mathrm{G} 01 *(\text { CDI_TOTA })+\mathrm{u} 0 \\
& \mathrm{~B} 1=\mathrm{G} 10+\mathrm{G} 11^{*}(\text { CDI_TOTA }) \\
& \mathrm{B} 2=\mathrm{G} 20+\mathrm{G} 21 *(\text { CDI_TOTA })+\mathrm{u} 2 \\
& \mathrm{~B} 3=\mathrm{G} 30+\mathrm{G} 31 *(\text { CDI_TOTA }) \\
& \mathrm{B} 4=\mathrm{G} 40 \\
& \mathrm{~B} 5=\mathrm{G} 50
\end{aligned}
$$

LG_SAA has been centered around the group mean.

CDI_TOTA has been centered around the grand mean.

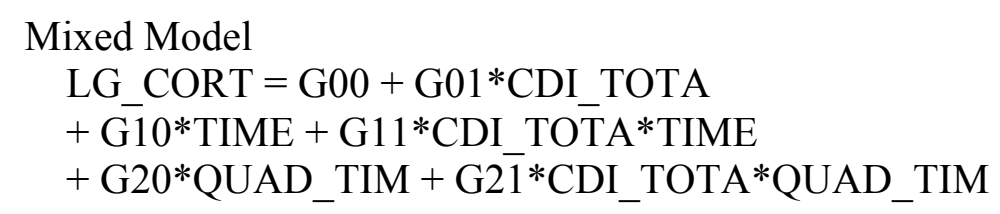




$$
\begin{aligned}
& + \text { G30*LG_SAA + G31*CDI_TOTA*LG_SAA } \\
& + \text { G40*SAAXTIME } \\
& + \text { G50*SAAXQ_TI } \\
& +\mathrm{u} 0+\mathrm{u} 2 * \text { QUAD_TIM + r }
\end{aligned}
$$

$\operatorname{sigma}^{\wedge} 2=0.01911$

Standard error of sigma^2 $=0.00241$

tau

INTRCPT1,B0 $0.03048 \quad-0.00246$

QUAD_TIM,B2 $-0.00246 \quad 0.00083$

Standard errors of tau

INTRCPT1,B0 $0.00759 \quad 0.00121$

QUAD_TIM,B2 $0.00121 \quad 0.00038$

tau (as correlations)

INTRCPT1,B0 $1.000-0.490$

QUAD_TIM,B2 -0.490 1.000

Random level-1 coefficient Reliability estimate

$\begin{array}{lc}\text { INTRCPT1, G0 } & 0.875 \\ \text { QUAD_TIM, G2 } & 0.494\end{array}$

\begin{tabular}{|c|c|c|c|c|c|}
\hline Fixed Effect & $\begin{array}{c}\text { Standard } \\
\text { Coefficient Erro }\end{array}$ & $\begin{array}{r}\text { Approx. } \\
\text { or } \quad \text { T-ratio }\end{array}$ & d.f. & P-value & \\
\hline \multicolumn{6}{|c|}{ For INTRCPT1, B0 } \\
\hline INTRCPT2, G00 & 0.449972 & 0.029649 & 15.177 & 40 & 0.000 \\
\hline CDI_TOTA, G01 & 0.003317 & 0.005032 & 0.659 & 40 & 0.514 \\
\hline \multicolumn{6}{|c|}{ For $\quad \overline{T I M E}$ slope, $\mathrm{B} 1$} \\
\hline INTRCPT2, G10 & -0.004705 & 0.008996 & -0.523 & 119 & 0.602 \\
\hline CDI_TOTA, G11 & -0.001633 & 0.001788 & -0.913 & 119 & 0.363 \\
\hline \multicolumn{6}{|c|}{ For QUAD_TIM slope, B2 } \\
\hline INTRCPT2, G20 & 0.012654 & 0.007398 & 1.711 & 40 & 0.095 \\
\hline CDI_TOTA, G21 & -0.000708 & 0.001237 & -0.573 & 40 & 0.570 \\
\hline
\end{tabular}

The value of the log-likelihood function at iteration $13=5.869653 \mathrm{E}+001$

The outcome variable is LG_CORT

Final estimation of fixed effects: 
$\begin{array}{llllll}\text { INTRCPT2, G30 } & -0.051002 & 0.125140 & -0.408 & 119 & 0.684\end{array}$

$\begin{array}{llllll}\text { CDI_TOTA, G31 } & -0.002178 & 0.015568 & -0.140 & 119 & 0.889\end{array}$

For SAAXTIME slope, B4

$\begin{array}{llllll}\text { INTRCPT2, G40 } & -0.052837 & 0.063870 & -0.827 & 119 & 0.410\end{array}$

For SAAXQ_TI slope, B5

$\begin{array}{llllll}\text { INTRCPT2, G50 } & -0.015418 & 0.049973 & -0.309 & 119 & 0.758\end{array}$

Final estimation of variance components:

Random Effect Standard Variance df Chi-square P-value Deviation Component

$\begin{array}{lllllll}\text { INTRCPT1, u0 } & 0.17458 & 0.03048 & 40 & 350.96008 & 0.000 \\ \text { QUAD_TIM slope, } \mathrm{u} 2 & 0.02882 & 0.00083 & 40 & 83.61745 & 0.000 \\ \text { level-1, r } & 0.13824 & 0.01911 & & & & \end{array}$

Statistics for the current model

Deviance $\quad=-117.393070$

Number of estimated parameters $=14$

Model comparison test

Chi-square statistic $=0.01889$

Degrees of freedom $=1$

$\mathrm{P}$-value $\quad=\quad>.500$

\section{Model 12}

Level-1 Model

LG_CORT $=$ B0 + B1 $*($ TIME $)+$ B2 $*\left(Q U A D \_T I M\right)+B 3 *\left(L G \_S A A\right)+B 4 *($ SAAXTIME $)+$ $\mathrm{B} 5 *(\mathrm{SAAX} \overline{\mathrm{X}} \mathrm{TI})+\mathrm{r}$

Level-2 Model

$$
\begin{aligned}
& \mathrm{B} 0=\mathrm{G} 00+\mathrm{G} 01 *(\mathrm{CDI} \text { TOTA })+\mathrm{u} 0 \\
& \mathrm{~B} 1=\mathrm{G} 10+\mathrm{G} 11 *(\mathrm{CDI} \text {-TOTA }) \\
& \mathrm{B} 2=\mathrm{G} 20+\mathrm{G} 21 *(\mathrm{CDI} \text { TOTA })+\mathrm{u} 2 \\
& \mathrm{~B} 3=\mathrm{G} 30+\mathrm{G} 31 *(\mathrm{CDI} \text { ITOTA }) \\
& \mathrm{B} 4=\mathrm{G} 40+\mathrm{G} 41 *(\mathrm{CDI} \text { ITOTA }) \\
& \mathrm{B} 5=\mathrm{G} 50+\mathrm{G} 51 *(\mathrm{CDI} \text { _TOTA })
\end{aligned}
$$

LG_SAA has been centered around the group mean.

CDI_TOTA has been centered around the grand mean. 


$$
\begin{aligned}
& \text { Mixed Model } \\
& \text { LG_CORT }=\mathrm{G} 00+\mathrm{G} 01 * \mathrm{CDI} \text { TOTA } \\
& + \text { G10*TIME + G11*CDI TOTA*TIME } \\
& + \text { G20*QUAD_TIM }+ \text { G2 } 1 \text { * CDI_TOTA*QUAD_TIM } \\
& + \text { G30*LG_SAA }+ \text { G31*CDI_TOTA*LG_SAA } \\
& \text { + G40*SAAXTIME + G41*CDI TOTA*SAAXTIME } \\
& + \text { G50*SAAXQ_TI }+ \text { G51*CDI_TOTA*SAAXQ_TI } \\
& +\mathrm{u} 0+\mathrm{u} 2 * \mathrm{QUAD} \text { TIM }+\mathrm{r}
\end{aligned}
$$

$\operatorname{sigma}^{\wedge} 2=0.01843$

Standard error of $\operatorname{sigma}^{\wedge} 2=0.00233$

tau

$$
\begin{array}{llc}
\text { INTRCPT1,B0 } & 0.03009 & -0.00249 \\
\text { QUAD_TIM,B2 } & -0.00249 & 0.00086
\end{array}
$$

Standard errors of tau

INTRCPT1,B0 $\quad 0.00747 \quad 0.00121$

\begin{tabular}{|c|c|c|c|c|c|}
\hline Fixed Effect & $\begin{array}{c}\text { Standard } \\
\text { Coefficient Erro }\end{array}$ & $\begin{array}{r}\text { Approx } \\
\text { or } \quad \text { T-ratic }\end{array}$ & d.f. & P-value & \\
\hline \multicolumn{6}{|c|}{ For INTRCPT1, B0 } \\
\hline INTRCPT2, G00 & 0.443110 & 0.029591 & 14.974 & 40 & 0.000 \\
\hline CDI TOTA, G01 & 0.000143 & 0.005196 & 0.028 & 40 & 0.978 \\
\hline \multicolumn{6}{|c|}{ For $\quad$ TIME slope, B1 } \\
\hline INTRCPT2, G10 & -0.007351 & 0.008986 & -0.818 & 117 & 0.415 \\
\hline
\end{tabular}

QUAD_TIM,B2 $0.00121 \quad 0.00038$

tau (as correlations)

INTRCPT1,B0 $1.000-0.490$

QUAD_TIM,B2 -0.490 1.000

Random level-1 coefficient Reliability estimate

$\begin{array}{lc}\text { INTRCPT1, G0 } & 0.877 \\ \text { QUAD_TIM, G2 } & 0.511\end{array}$

The value of the log-likelihood function at iteration $13=6.137541 \mathrm{E}+001$ The outcome variable is LG_CORT

Final estimation of fixed effects: 
$\begin{array}{llllll}\text { CDI_TOTA, G11 } & -0.002950 & 0.001915 & -1.541 & 117 & 0.126\end{array}$

For QŪAD_TIM slope, B2

$\begin{array}{llllll}\text { INTRCPT2, G20 } & 0.009575 & 0.007493 & 1.278 & 40 & 0.209\end{array}$

$\begin{array}{llllll}\text { CDI_TOTA, G21 } & -0.002095 & 0.001371 & -1.528 & 40 & 0.134\end{array}$

For LG_SAA slope, B3

$\begin{array}{llllll}\text { INTRCPT2, G30 } & 0.035312 & 0.129246 & 0.273 & 117 & 0.785\end{array}$

$\begin{array}{llllll}\text { CDI_TOTA, G31 } & 0.037616 & 0.023245 & 1.618 & 117 & 0.108\end{array}$

For SAAXTIME slope, B4

$\begin{array}{llllll}\text { INTRCPT2, G40 } & 0.001502 & 0.067597 & 0.022 & 117 & 0.982\end{array}$

$\begin{array}{llllll}\text { CDI_TOTA, G41 } & 0.023342 & 0.011502 & 2.029 & 117 & 0.045\end{array}$

For SÄAXQ_TI slope, B5

$\begin{array}{llllll}\text { INTRCPT2, G50 } & -0.013971 & 0.050404 & -0.277 & 117 & 0.782\end{array}$

CDI_TOTA, G51 $\quad-0.002090 \quad 0.007537 \quad-0.277 \quad 117 \quad 0.782$

Final estimation of variance components:

Random Effect Standard Variance df Chi-square P-value Deviation Component

$\begin{array}{lllllll}\text { INTRCPT1, u0 } & 0.17347 & 0.03009 & 40 & 358.35600 & 0.000\end{array}$

QUAD_TIM slope, u2 $\quad 0.02931 \quad 0.00086 \quad 40 \quad 86.78991 \quad 0.000$

$\begin{array}{llll}\text { level-1, } & \mathrm{r} & 0.13577 & 0.01843\end{array}$

Statistics for the current model

Deviance $=-122.750812$

Number of estimated parameters $=16$

Model comparison test

Chi-square statistic $=5.35774$

Degrees of freedom $=2$

P-value $\quad=0.067$

\section{Model 13}

Level-1 Model

LG_CORT $=$ B0 + B $1 *($ TIME $)+$ B2 $*\left(Q U A D \_T I M\right)+B 3 *\left(L G \_S A A\right)+B 4 *(S A A X T I M E)+r$

Level-2 Model

$$
\begin{aligned}
& \mathrm{B} 0=\mathrm{G} 00+\mathrm{G} 01 *(\mathrm{CDI} \text { TOTA })+\mathrm{u} 0 \\
& \mathrm{~B} 1=\mathrm{G} 10+\mathrm{G} 11 *(\text { CDI_TOTA }) \\
& \mathrm{B} 2=\mathrm{G} 20+\mathrm{G} 21 *(\text { CDI_TOTA })+\mathrm{u} 2
\end{aligned}
$$




$$
\begin{aligned}
& \mathrm{B} 3=\mathrm{G} 30+\mathrm{G} 31 *(\mathrm{CDI} \text { ITOTA }) \\
& \mathrm{B} 4=\mathrm{G} 40+\mathrm{G} 41 *(\mathrm{CDI} \text { _TOTA })
\end{aligned}
$$

LG_SAA has been centered around the group mean.

CDI_TOTA has been centered around the grand mean.

$$
\begin{aligned}
& \text { Mixed Model } \\
& \text { LG_CORT }=\mathrm{G} 00+\mathrm{G} 01 * \text { CDI_TOTA } \\
& +\overline{\mathrm{G}} 10 * \mathrm{TIME}+\mathrm{G} 11 * \mathrm{CDI} \text { TOTA*TIME } \\
& + \text { G20*QUAD_TIM }+ \text { G2 } 1 \text { *CDI_TOTA*QUAD_TIM } \\
& + \text { G30*LG_SAA }+ \text { G3 } 1 * \text { CDI_TOTA*LG_SAA }
\end{aligned}
$$

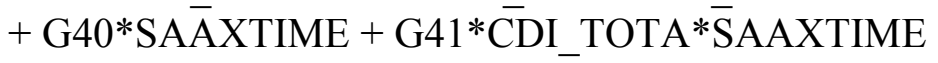

$$
\begin{aligned}
& +\mathrm{u} 0+\mathrm{u} 2 * \mathrm{QUAD} \text { TIM }+\mathrm{r}
\end{aligned}
$$

$\operatorname{sigma}^{\wedge} 2=0.01842$

Standard error of $\operatorname{sigma}^{\wedge} 2=0.00232$

tau

$$
\begin{array}{llc}
\text { INTRCPT1,B0 } & 0.03008 & -0.00244 \\
\text { QUAD_TIM,B2 } & -0.00244 & 0.00086
\end{array}
$$

Standard errors of tau

INTRCPT1,B0 $\quad 0.00747 \quad 0.00121$

QUAD_TIM,B2 $0.00121 \quad 0.00038$

tau (as correlations)

INTRCPT1,B0 $1.000-0.479$

QUAD_TIM,B2 -0.479 1.000

Random level-1 coefficient Reliability estimate

$\begin{array}{lc}\text { INTRCPT1, G0 } & 0.877 \\ \text { QUAD_TIM, G2 } & 0.513\end{array}$

The value of the log-likelihood function at iteration $9=6.131260 \mathrm{E}+001$ The outcome variable is LG_CORT

Final estimation of fixed effects:

Fixed Effect Coefficient Error T-ratio d.f. P-value


For INTRCPT1, B0

$\begin{array}{llllll}\text { INTRCPT2, G00 } & 0.443948 & 0.029493 & 15.053 & 40 & 0.000\end{array}$

$\begin{array}{llllll}\text { CDI_TOTA, G01 } & 0.000366 & 0.005153 & 0.071 & 40 & 0.944\end{array}$

For TIME slope, B1

$\begin{array}{llllll}\text { INTRCPT2, G10 } & -0.007746 & 0.008909 & -0.869 & 119 & 0.386\end{array}$

$\begin{array}{llllll}\text { CDI_TOTA, G11 } & -0.003126 & 0.001846 & -1.693 & 119 & 0.093\end{array}$

For QUAD_TIM slope, B2

$\begin{array}{llllll}\text { INTRCPT2, G20 } & 0.009619 & 0.007479 & 1.286 & 40 & 0.206\end{array}$

$\begin{array}{llllll}\text { CDI_TOTA, G21 } & -0.002130 & 0.001368 & -1.557 & 40 & 0.127\end{array}$

For LG_SAA slope, B3

$\begin{array}{llllll}\text { INTRCPT2, G30 } & 0.039368 & 0.128318 & 0.307 & 119 & 0.760\end{array}$

$\begin{array}{llllll}\text { CDI_TOTA, G31 } & 0.038031 & 0.023283 & 1.633 & 119 & 0.105\end{array}$

For SAAXTIME slope, B4

$\begin{array}{llllll}\text { INTRCPT2, G40 } & 0.010962 & 0.057375 & 0.191 & 119 & 0.849\end{array}$

$\begin{array}{llllll}\text { CDI_TOTA, G41 } & 0.024689 & 0.010618 & 2.325 & 119 & 0.022\end{array}$

Final estimation of variance components:

Random Effect Standard Variance df Chi-square P-value Deviation Component

$\begin{array}{lllllll}\text { INTRCPT1, u0 } & 0.17343 & 0.03008 & 40 & 358.47538 & 0.000\end{array}$

QUAD_TIM slope, u2 $\quad 0.02941 \quad 0.00086 \quad 40 \quad 87.09009 \quad 0.000$ $\begin{array}{llll}\text { level-1, } & \mathrm{r} & 0.13571 & 0.01842\end{array}$

Statistics for the current model

Deviance $\quad=-122.625198$

Number of estimated parameters $=14$

Model comparison test

Chi-square statistic $=5.23213$

Degrees of freedom $=0$

$\mathrm{P}$-value $\quad=\quad>.500$

\section{Model 14}

Level-1 Model

$$
\mathrm{LG} \_\mathrm{CORT}=\mathrm{B} 0+\mathrm{B} 1 *(\mathrm{TIME})+\mathrm{B} 2 *\left(\mathrm{QUAD} \_\mathrm{TIM}\right)+\mathrm{B} 3 *\left(\mathrm{LG}_{-} \mathrm{SAA}\right)+\mathrm{B} 4 *(\mathrm{SAAXTIME})+\mathrm{r}
$$

Level-2 Model

$$
\mathrm{B} 0=\mathrm{G} 00+\mathrm{G} 01 *(\mathrm{CDI} \text { TOTA })+\mathrm{u} 0
$$




$$
\begin{aligned}
& \mathrm{B} 1=\mathrm{G} 10+\mathrm{G} 11 *(\mathrm{CDI} \text { TOTA }) \\
& \mathrm{B} 2=\mathrm{G} 20+\mathrm{u} 2 \\
& \mathrm{~B} 3=\mathrm{G} 30+\mathrm{G} 31 *(\mathrm{CDI} \text { TOTA }) \\
& \mathrm{B} 4=\mathrm{G} 40
\end{aligned}
$$

LG_SAA has been centered around the group mean.

CDI_TOTA has been centered around the grand mean.

$$
\begin{aligned}
& \text { Mixed Model } \\
& \text { LG_CORT }=\text { G00 + G01*CDI_TOTA } \\
& \text { + G10*TIME + G1 } 1 * \text { CDI_TOTA*TIME } \\
& \text { + G20*QUAD_TIM } \\
& \text { + G30*LG_SAA }+ \text { G3 } 31 * \text { CDI_TOTA*LG_SAA } \\
& \text { + G40*SAAXTIME } \\
& + \text { u } 0+\mathrm{u} 2 * \text { QUAD_TIM }+\mathrm{r}
\end{aligned}
$$

$\operatorname{sigma}^{\wedge} 2=0.01906$

Standard error of $\operatorname{sigma}^{\wedge} 2=0.00241$

$$
\begin{array}{llc}
\text { tau } & & \\
\text { INTRCPT1,B0 } & 0.03043 & -0.00240 \\
\text { QUAD_TIM,B2 } & -0.00240 & 0.00086
\end{array}
$$

Standard errors of tau

INTRCPT1,B0 $0.00757 \quad 0.00122$

QUAD_TIM,B2 $0.00122 \quad 0.00038$

tau (as correlations)

INTRCPT1,B0 $1.000-0.470$

QUAD_TIM,B2 -0.470 1.000

Random level-1 coefficient Reliability estimate

\begin{tabular}{lc}
\hline INTRCPT1, G0 & 0.875 \\
QUAD_TIM, G2 & 0.502
\end{tabular}

The value of the log-likelihood function at iteration $8=5.847700 \mathrm{E}+001$

The outcome variable is LG_CORT

Final estimation of fixed effects:

$$
\text { Standard Approx. }
$$




$$
\begin{aligned}
& \mathrm{B} 1=\mathrm{G} 10+\mathrm{G} 11 *(\mathrm{CDI} \text { ITOTA }) \\
& \mathrm{B} 2=\mathrm{G} 20+\mathrm{u} 2 \\
& \mathrm{~B} 3=\mathrm{G} 30+\mathrm{G} 31 *(\mathrm{CDI} \text { TOTA }) \\
& \mathrm{B} 4=\mathrm{G} 40+\mathrm{G} 41 *(\mathrm{CDI} \text { _TOTA })
\end{aligned}
$$

LG_SAA has been centered around the group mean.

CDI_TOTA has been centered around the grand mean.

$$
\begin{aligned}
& \text { Mixed Model } \\
& \text { LG_CORT }=\text { G00 + G0 } 1 * \text { CDI_TOTA } \\
& \text { + G10*TIME + G11*CDI_TOTA*TIME } \\
& \text { + G20*QUAD_TIM } \\
& \text { + G30*LG_SAA + G3 } 1 * \text { CDI_TOTA*LG_SAA } \\
& \text { + G40*SAAXTIME + G4 } 1 * \text { CDI_TOTA*SAAXTIME } \\
& \text { + u0 + u } 2 * \text { QUAD_TIM + r }
\end{aligned}
$$

$\operatorname{sigma}^{\wedge} 2=0.01857$

Standard error of $\operatorname{sigma}^{\wedge} 2=\quad 0.00234$

$$
\begin{array}{lcc}
\text { tau } & & \\
\text { INTRCPT1,B0 } & 0.02996 & -0.00251 \\
\text { QUAD_TIM,B2 } & -0.00251 & 0.00092
\end{array}
$$

Standard errors of tau

$$
\begin{array}{lcc}
\text { INTRCPT1,B0 } & 0.00745 & 0.00123 \\
\text { QUAD_TIM,B2 } & 0.00123 & 0.00039
\end{array}
$$

tau (as correlations)

INTRCPT1,B0 $1.000-0.478$

QUAD_TIM,B2 -0.478 1.000

Random level-1 coefficient Reliability estimate

$\begin{array}{lc}\text { INTRCPT1, G0 } & 0.876 \\ \text { QUAD_TIM, G2 } & 0.526\end{array}$

The value of the log-likelihood function at iteration $9=6.011621 \mathrm{E}+001$

The outcome variable is LG_CORT

Final estimation of fixed effects: 


\begin{tabular}{|c|c|c|c|c|c|}
\hline Fixed Effect & $\begin{array}{c}\text { Standard } \\
\text { Coefficient Erro }\end{array}$ & $\begin{array}{r}\text { Approx } \\
\text { or } \quad \text { T-ratic }\end{array}$ & d.f. & P-value & \\
\hline \multicolumn{6}{|c|}{ For INTRCPT1, B0 } \\
\hline INTRCPT2, G00 & 0.445658 & 0.029448 & 15.134 & 40 & 0.000 \\
\hline CDI_TOTA, G01 & -0.001018 & 0.005072 & -0.201 & 40 & 0.842 \\
\hline \multicolumn{6}{|c|}{ For TIME slope, B1 } \\
\hline INTRCPT2, G10 & .006662 & 0.008919 & -0.747 & 119 & 0.457 \\
\hline CDI_TOTA, G11 & -0.001619 & 0.001575 & -1.028 & 119 & 0.306 \\
\hline \multicolumn{6}{|c|}{ For QŪAD_TIM slope, B2 } \\
\hline INTRCPT2, G20 & 0.011043 & 0.007532 & 1.466 & 41 & 0.150 \\
\hline \multicolumn{6}{|c|}{ For LG_SAA slope, B3 } \\
\hline INTRCPT2, G30 & -0.000627 & 0.127035 & -0.005 & 119 & 0.996 \\
\hline CDI_TOTA, G31 & 0.027745 & 0.022477 & 1.234 & 119 & 0.219 \\
\hline \multicolumn{6}{|c|}{ For SĀAXTIME slope, B4 } \\
\hline INTRCPT2, G40 & -0.012773 & 0.05 & $-0.2-2$ & 119 & 0.819 \\
\hline CDI_TOTA, G41 & 0.017577 & 0.009605 & 1.830 & 119 & 0.070 \\
\hline
\end{tabular}

Final estimation of variance components:

Random Effect Standard Variance df Chi-square P-value Deviation Component

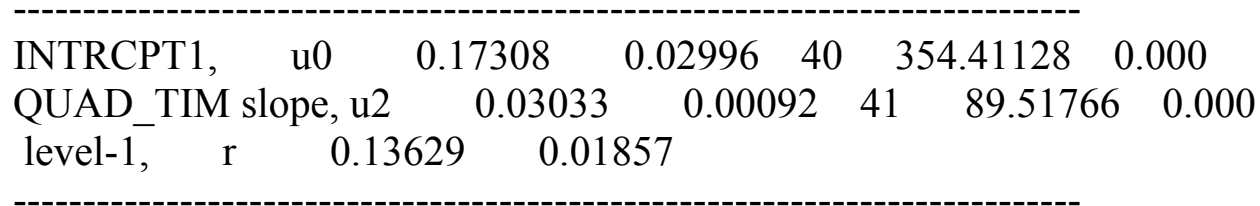

Statistics for the current model

Deviance $\quad=-120.232413$

Number of estimated parameters $=13$

Model comparison test

$$
\begin{aligned}
& \text { Chi-square statistic }=3.27841 \\
& \text { Degrees of freedom }=1 \\
& \mathrm{P} \text {-value } \quad=0.067
\end{aligned}
$$

\section{Model 16}

Level-1 Model

$$
\text { LG_CORT }=\text { B0 }+ \text { B } 1 *(\text { TIME })+\text { B2 } *(\text { QUAD_TIM })+\text { B } 3 *\left(L G \_S A A\right)+r
$$


Level-2 Model

$$
\begin{aligned}
& \mathrm{B} 0=\mathrm{G} 00+\mathrm{G} 01 *(\mathrm{CDI} \text { IOTA })+\mathrm{u} 0 \\
& \mathrm{~B} 1=\mathrm{G} 10+\mathrm{G} 11 *(\mathrm{CDI} \text { _TOTA }) \\
& \mathrm{B} 2=\mathrm{G} 20+\mathrm{u} 2 \\
& \mathrm{~B} 3=\mathrm{G} 30+\mathrm{G} 31 *(\mathrm{CDI} \text { _TOTA })
\end{aligned}
$$

LG_SAA has been centered around the group mean.

CDI_TOTA has been centered around the grand mean.

$$
\begin{aligned}
& \text { Mixed Model } \\
& \text { LG_CORT }=\mathrm{G} 00+\mathrm{G} 01 * \text { CDI_TOTA } \\
& + \text { G10*TIME }+ \text { G } 11 * \text { CDI_TOTA*TIME } \\
& + \text { G20*QUAD_TIM } \\
& + \text { G30*LG_SAA + G } 31 * \text { CDI_TOTA*LG_SAA } \\
& +\mathrm{u} 0+\mathrm{u} 2 * \text { QUAD_TIM }+\mathrm{r}
\end{aligned}
$$

$\operatorname{sigma}^{\wedge} 2=0.01921$

Standard error of $\operatorname{sigma}^{\wedge} 2=0.00242$

$$
\begin{array}{llc}
\text { tau } & & \\
\text { INTRCPT1,B0 } & 0.03100 & -0.00252 \\
\text { QUAD_TIM,B2 } & -0.00252 & 0.00083
\end{array}
$$

Standard errors of tau

$\begin{array}{lll}\text { INTRCPT1,B0 } & 0.00770 & 0.00122\end{array}$

QUAD_TIM,B2 $0.00122 \quad 0.00038$

tau (as correlations)

INTRCPT1,B0 $1.000-0.497$

QUAD_TIM,B2 -0.497 1.000

Random level-1 coefficient Reliability estimate

\begin{tabular}{lc}
\hline INTRCPT1, G0 & 0.876 \\
QUAD_TIM, G2 & 0.491 \\
\hline-----
\end{tabular}

The value of the log-likelihood function at iteration $12=5.811156 \mathrm{E}+001$

The outcome variable is LG_CORT

Final estimation of fixed effects: 


\begin{tabular}{|c|c|c|c|c|c|}
\hline Fixed Effect & $\begin{array}{c}\text { Standard } \\
\text { Coefficient Erro }\end{array}$ & $\begin{array}{r}\text { Approx. } \\
\text { or T-ratio }\end{array}$ & d.f. & P-value & \\
\hline \multicolumn{6}{|c|}{ For INTRCPT1, B0 } \\
\hline INTRCPT2, G00 & 0.448875 & 0.029778 & 15.074 & 40 & 0.000 \\
\hline CDI_TOTA, G0 & 0.002077 & 0.004763 & 0.436 & 40 & 0.665 \\
\hline \multicolumn{6}{|c|}{ For TIME slope, B1 } \\
\hline INTRCPT2, G10 & -0.004799 & 0.009005 & -0.533 & 121 & 0.595 \\
\hline CDI_TOTA, G1 & -0.001304 & 0.001567 & -0.832 & 121 & 0.407 \\
\hline \multicolumn{6}{|c|}{ For QUAD_TIM slope, B2 } \\
\hline INTRCPT2, G20 & 0.012380 & 0.007334 & 1.688 & 41 & 0.099 \\
\hline \multicolumn{6}{|c|}{ For LG_SAA slope, B3 } \\
\hline INTRCPT2, G30 & 0.006434 & 0.104114 & 0.062 & 121 & 0.951 \\
\hline CDI_TOTA, G3 & -0.001690 & 0.015538 & -0.109 & 121 & 0.914 \\
\hline
\end{tabular}

Final estimation of variance components:

Random Effect Standard Variance df Chi-square P-value Deviation Component

$\begin{array}{lllllll}\text { INTRCPT1, u0 } & 0.17606 & 0.03100 & 40 & 354.64899 & 0.000\end{array}$

QUAD_TIM slope, u2 $\quad 0.02874 \quad 0.00083 \quad 41 \quad 83.18240 \quad 0.000$ $\begin{array}{llll}\text { level-1, } & \mathrm{r} & 0.13858 & 0.01921\end{array}$

Statistics for the current model

Deviance $\quad=-116.223124$

Number of estimated parameters $=11$

\section{Model 17}

Level-1 Model

$$
\text { LG_CORT }=\text { B0 }+ \text { B1 } *(\text { TIME })+\text { B2 } *\left(Q U A D \_T I M\right)+B 3 *\left(L G \_S A A\right)+r
$$

Level-2 Model

$$
\begin{aligned}
& \mathrm{B} 0=\mathrm{G} 00+\mathrm{G} 01 *(\mathrm{CDI} \text { TOTA })+\mathrm{u} 0 \\
& \mathrm{~B} 1=\mathrm{G} 10 \\
& \mathrm{~B} 2=\mathrm{G} 20+\mathrm{u} 2 \\
& \mathrm{~B} 3=\mathrm{G} 30
\end{aligned}
$$

LG_SAA has been centered around the group mean.

CDI_TOTA has been centered around the grand mean. 


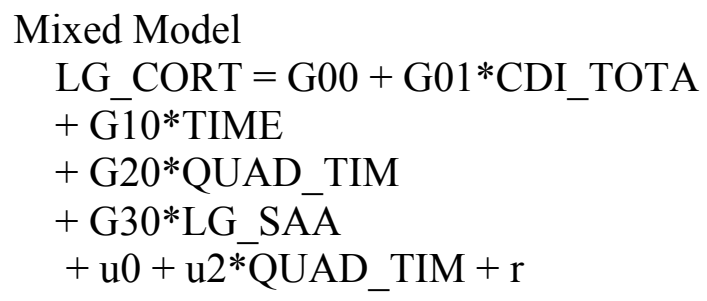

$\operatorname{sigma}^{\wedge} 2=0.01942$

Standard error of sigma ${ }^{\wedge} 2=0.00245$

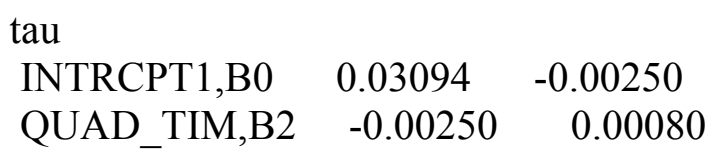

Standard errors of tau

INTRCPT1,B0 $\quad 0.00770 \quad 0.00122$

QUAD_TIM,B2 $0.00122 \quad 0.00038$

tau (as correlations)

INTRCPT1,B0 $1.000-0.504$

QUAD_TIM,B2 -0.504 1.000

Random level-1 coefficient Reliability estimate

$\begin{array}{lc}\text { INTRCPT1, G0 } & 0.875 \\ \text { QUAD_TIM, G2 } & 0.480\end{array}$

The value of the log-likelihood function at iteration $8=5.767045 \mathrm{E}+001$

The outcome variable is LG_CORT

Final estimation of fixed effects:

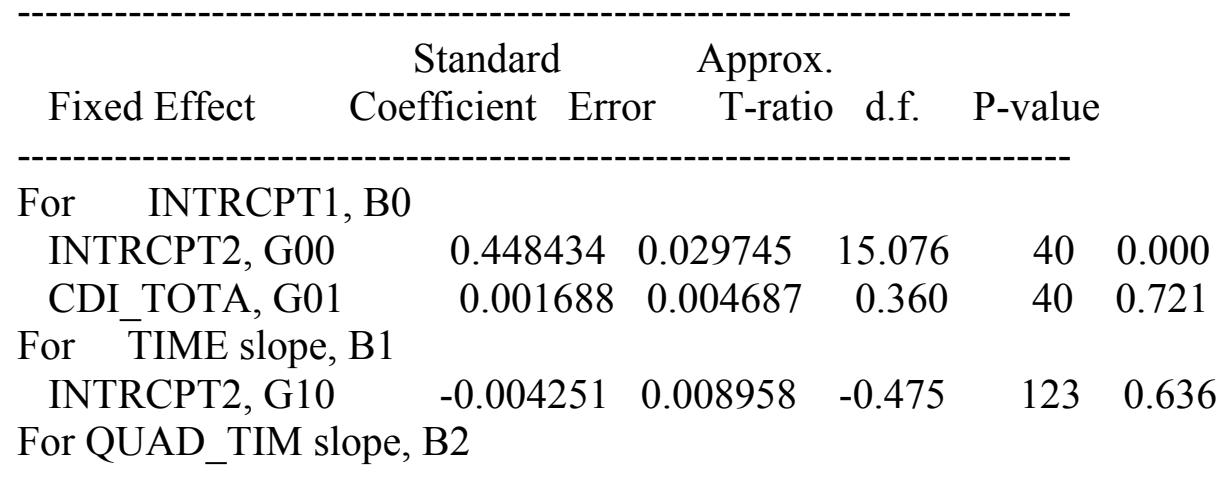


$\begin{array}{llllll}\text { INTRCPT2, G20 } & 0.012297 & 0.007307 & 1.683 & 41 & 0.100\end{array}$

For LG_SAA slope, B3

$\begin{array}{llllll}\text { INTRCPT2, G30 } & 0.022427 & 0.100915 & 0.222 & 123 & 0.825\end{array}$

The outcome variable is LG_CORT

Final estimation of variance components:

Random Effect Standard Variance df Chi-square P-value Deviation Component

$\begin{array}{lllllll}\text { INTRCPT1, } & \mathrm{u} 0 & 0.17589 & 0.03094 & 40 & 350.63349 & 0.000\end{array}$

QUAD_TIM slope, u2 $\quad 0.02823 \quad 0.00080 \quad 41 \quad 81.26064 \quad 0.000$ $\begin{array}{llll}\text { level-1, } & \mathrm{r} & 0.13935 & 0.01942\end{array}$

Statistics for the current model

Deviance $\quad=-115.340903$

Number of estimated parameters $=9$

Model 18

Level-1 Model

$$
\text { LG_CORT }=\text { B0 }+ \text { B } 1 *(\text { TIME })+\text { B2 } *\left(Q U A D \_T I M\right)+B 3 *\left(L G \_S A A\right)+r
$$

Level-2 Model

$$
\begin{aligned}
& \mathrm{B} 0=\mathrm{G} 00+\mathrm{u} 0 \\
& \mathrm{~B} 1=\mathrm{G} 10 \\
& \mathrm{~B} 2=\mathrm{G} 20+\mathrm{u} 2 \\
& \mathrm{~B} 3=\mathrm{G} 30
\end{aligned}
$$

LG_SAA has been centered around the group mean.

$$
\begin{aligned}
& \text { Mixed Model } \\
& \text { LG_CORT }=\text { G00 } \\
& + \text { G10*TIME } \\
& + \text { G20*QUAD_TIM } \\
& + \text { G30*LG_SAA } \\
& +\mathrm{u} 0+\mathrm{u} 2 * \text { QUAD_TIM }+\mathrm{r}
\end{aligned}
$$

$\operatorname{sigma}^{\wedge} 2=0.01942$ 
Standard error of sigma^2 ${ }^{\wedge} \quad 0.00245$

tau

INTRCPT1,B0 $0.03106 \quad-0.00251$

QUAD_TIM,B2 $\quad-0.00251 \quad 0.00080$

Standard errors of tau

$\begin{array}{lll}\text { INTRCPT1,B0 } & 0.00773 & 0.00122\end{array}$

QUAD_TIM,B2 $0.00122 \quad 0.00038$

tau (as correlations)

INTRCPT1,B0 $1.000-0.504$

QUAD_TIM,B2 -0.504 1.000

Random level-1 coefficient Reliability estimate

$\begin{array}{lc} & \\ \text { INTRCPT1, G0 } & 0.875 \\ \text { QUAD_TIM, G2 } & 0.479\end{array}$

The value of the $\log$-likelihood function at iteration $8=5.760570 \mathrm{E}+001$

The outcome variable is LG_CORT

Final estimation of fixed effects:

\begin{tabular}{|c|c|c|c|c|c|}
\hline Fixed Effect & $\begin{array}{c}\text { Standard } \\
\text { Coefficient Error }\end{array}$ & $\begin{array}{r}\text { Approx. } \\
\text { T-ratio }\end{array}$ & d.f. & P-value & \\
\hline \multicolumn{6}{|c|}{ For INTRCPT1, B0 } \\
\hline INTRCPT2, G0C & $0.448048 \quad 0$ & 0.029773 & 15.049 & 41 & 0.000 \\
\hline \multicolumn{6}{|c|}{ For TIME slope, B1 } \\
\hline INTRCPT2, G1C & $-0.004231 \quad 0$ & 0.008958 & -0.472 & 123 & 0.638 \\
\hline \multicolumn{6}{|c|}{ For QUAD_TIM slope, B2 } \\
\hline INTRCPT2, G2C & $0.012288 \quad 0$ & 0.007306 & 1.682 & 41 & 0.100 \\
\hline \multicolumn{6}{|c|}{ For LG SAA slope, B3 } \\
\hline INTRCPT2, G3C & $0.023959 \quad 0$ & 0.100829 & 0.238 & 123 & 0.813 \\
\hline
\end{tabular}

Final estimation of variance components:

Random Effect Standard Variance df Chi-square P-value Deviation Component

$\begin{array}{llllll}\text { INTRCPT1, u0 } & 0.17623 & 0.03106 & 41 & 351.79917 & 0.000\end{array}$

QUAD_TIM slope, u2 $\quad 0.02822 \quad 0.00080 \quad 41 \quad 81.23429 \quad 0.000$ 
$\begin{array}{llll}\text { level-1, } & \mathrm{r} & 0.13935 & 0.01942\end{array}$

Statistics for the current model

Deviance $\quad=-115.211409$

Number of estimated parameters $=8$

Model comparison test

Chi-square statistic $=14.09680$

Degrees of freedom $=2$

$\mathrm{P}$-value $\quad=0.001$

\section{Model 19}

Level-1 Model

$$
\mathrm{LG} \_\mathrm{CORT}=\mathrm{B} 0+\mathrm{B} 1 *(\mathrm{TIME})+\mathrm{B} 2 *\left(\mathrm{QUAD} \_\mathrm{TIM}\right)+\mathrm{r}
$$

Level-2 Model

$$
\begin{aligned}
& \mathrm{B} 0=\mathrm{G} 00+\mathrm{u} 0 \\
& \mathrm{~B} 1=\mathrm{G} 10 \\
& \mathrm{~B} 2=\mathrm{G} 20+\mathrm{u} 2
\end{aligned}
$$

Mixed Model

LG_CORT $=$ G00

+ G10*TIME

$+\mathrm{G} 20 * \mathrm{QUAD}$-TIM $+\mathrm{u} 0+\mathrm{u} 2 * \mathrm{QUAD}$ TIM $+\mathrm{r}$

$\operatorname{sigma}^{\wedge} 2=0.02223$

Standard error of $\operatorname{sigma}^{\wedge} 2=\quad 0.00232$

tau

INTRCPT1,B0 $0.04582 \quad-0.00066$

QUAD_TIM,B2 -0.00066 0.00021

Standard errors of tau

INTRCPT1,B0 $0.01033 \quad 0.00095$

QUAD_TIM,B2 $0.00095 \quad 0.00018$

tau (as correlations)

INTRCPT1,B0 $1.000-0.212$

QUAD_TIM,B2 -0.212 1.000 
Random level-1 coefficient Reliability estimate

INTRCPT1, G0 $\quad 0.925$

QUAD_TIM, G2 $\quad 0.257$

The value of the log-likelihood function at iteration $2=6.747734 \mathrm{E}+001$

The outcome variable is LG_CORT

Final estimation of fixed effects:

\begin{tabular}{|c|c|c|c|c|c|}
\hline Fixed Effect & $\begin{array}{c}\text { Standard } \\
\text { Coefficient Erro }\end{array}$ & $\begin{array}{r}\text { Approx. } \\
\text { or } \quad \text { T-ratio }\end{array}$ & d.f. & P-value & \\
\hline \multicolumn{6}{|c|}{ For INTRCPT1, B0 } \\
\hline \multicolumn{6}{|c|}{ For TIME slope, B1 } \\
\hline INTRCPT2, G & -0.000188 & 0.005254 & -0.036 & 183 & 0.972 \\
\hline \multicolumn{6}{|c|}{ For QUAD_TIM slope, B2 } \\
\hline INTRCPT2, G & 0.014040 & 0.004248 & 3.305 & 45 & 0.002 \\
\hline
\end{tabular}

Final estimation of variance components:

Random Effect Standard Variance df Chi-square P-value Deviation Component

$\begin{array}{lllllll}\text { INTRCPT1, u0 } & 0.21405 & 0.04582 & 45 & 614.85525 & 0.000 \\ \text { QUAD_TIM slope, u2 } & 0.01460 & 0.00021 & 45 & 61.87902 & 0.048 \\ \text { level-1, r r } \quad 0.14908 & 0.02223 & & & & \end{array}$

Statistics for the current model

Deviance $\quad=-134.954687$

Number of estimated parameters $=7$

Model comparison test

Chi-square statistic $=19.74328$

Degrees of freedom $=1$

$\mathrm{P}$-value $\quad=\quad<0.001$

Model 20 - covariates 
Level-1 Model

$$
\text { LG_CORT }=\text { B } 0+\text { B } 1 *(T I M E)+B 2 *\left(Q U A D \_T I M\right)+r
$$

Level-2 Model

$$
\mathrm{B} 0=\mathrm{G} 00+\mathrm{G} 01 *(\mathrm{CHILD} E \mathrm{EA})+\mathrm{G} 02 *(\mathrm{CHILD} \text {-WA })+\mathrm{G} 03 *\left(\mathrm{CHILD} \_\mathrm{SE}\right)+
$$
G04*(CHILD_AG)

+ G05*(ETHNICIT $)+$ G06*(TIME_GAP $)+u 0$

$\mathrm{B} 1=\mathrm{G} 10$

$\mathrm{B} 2=\mathrm{G} 20+\mathrm{u} 2$

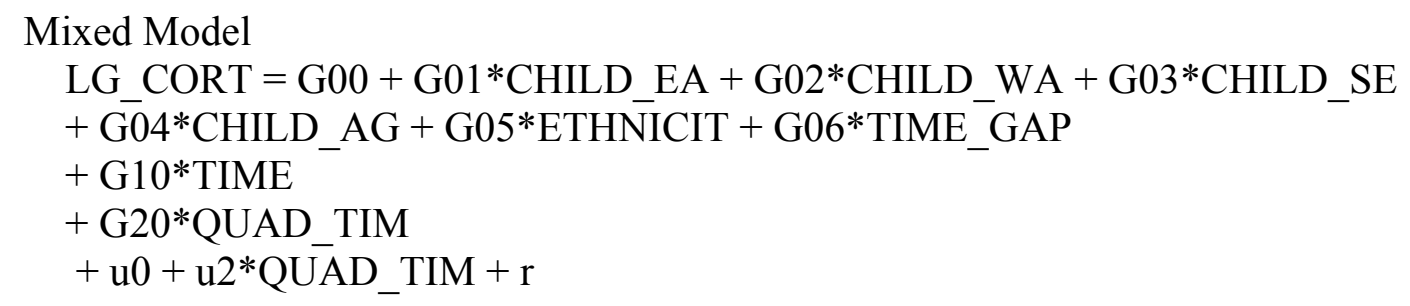

$\operatorname{sigma}^{\wedge} 2=0.02243$

tau

INTRCPT1,B0 $0.04323 \quad-0.00045$

QUAD_TIM,B2 $\quad-0.00045 \quad 0.00029$

tau (as correlations)

INTRCPT1,B0 $1.000-0.127$

QUAD_TIM,B2 -0.127 1.000

Random level-1 coefficient Reliability estimate

\begin{tabular}{lc}
\hline INTRCPT1, G0 & 0.920 \\
QUAD_TIM, G2 & 0.319
\end{tabular}

The value of the log-likelihood function at iteration $17=2.093882 \mathrm{E}+001$

\begin{tabular}{|c|c|c|c|c|}
\hline Fixed Effect & $\begin{array}{c}\text { Standard } \\
\text { Coefficient Error }\end{array}$ & $\begin{array}{r}\text { Approx. } \\
\text { T-ratio }\end{array}$ & d.f. & P-value \\
\hline INTR & & & & \\
\hline $\begin{array}{l}\text { INTRCPT2, } \\
\text { CHILD EA }\end{array}$ & $\begin{array}{c}0.7706120 \\
0.000004\end{array}$ & .572080 & $\begin{array}{l}1.347 \\
0.436\end{array}$ & $\begin{array}{c}34 \\
34\end{array}$ \\
\hline
\end{tabular}

Final estimation of fixed effects: 


$\begin{array}{lccccc}\text { CHILD_WA, G02 } & 0.000000 & 0.000012 & 0.027 & 34 & 0.979 \\ \text { CHILD_SE, G03 } & 0.023276 & 0.074102 & 0.314 & 34 & 0.755 \\ \text { CHILD_AG, G04 } & -0.049399 & 0.053201 & -0.929 & 34 & 0.360 \\ \text { ETHNICIT, G05 } & -0.002131 & 0.014019 & -0.152 & 34 & 0.880 \\ \text { TIME_GAP, G06 } & -0.030992 & 0.016097 & -1.925 & 34 & 0.063 \\ \begin{array}{c}\text { For TIME slope, B1 } \\ \quad\end{array} & & & & & \\ \begin{array}{l}\text { INTRCPT2, G10 } \\ \text { For QUAD_TIM slope, B2 }\end{array} & 0.005461 & 0.005591 & 0.977 & 163 & 0.330 \\ \begin{array}{l}\text { INTRCPT2, G20 } \\ \text { INTRE }\end{array} & 0.013332 & 0.004724 & 2.822 & 40 & 0.007\end{array}$

Final estimation of variance components:

Random Effect Standard Variance df Chi-square P-value Deviation Component

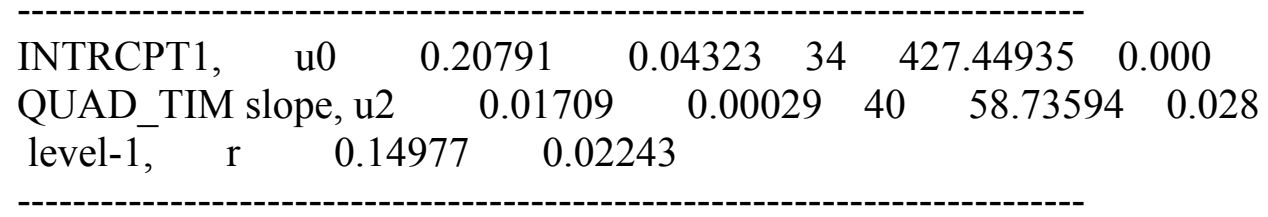

Statistics for current covariance components model

Deviance $\quad=-41.877641$

Number of estimated parameters $=4$

\section{Model 21- Final model}

Level-1 Model

$$
\text { LG_CORT }=\text { B } 0+\text { B } 1 *(\text { TIME })+\text { B2 } *\left(Q U A D \_T I M\right)+r
$$

Level-2 Model

$$
\begin{aligned}
& \mathrm{B} 0=\mathrm{G} 00+\mathrm{u} 0 \\
& \mathrm{~B} 1=\mathrm{G} 10 \\
& \mathrm{~B} 2=\mathrm{G} 20+\mathrm{u} 2
\end{aligned}
$$

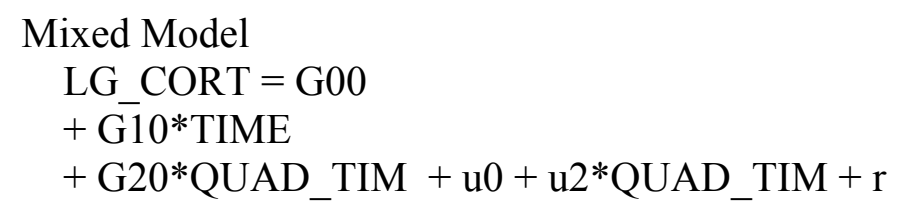

$\operatorname{sigma}^{\wedge} 2=0.02235$

tau 


$\begin{array}{llc}\text { INTRCPT1,B0 } & 0.04690 & -0.00068 \\ \text { QUAD_TIM,B2 } & -0.00068 & 0.00023\end{array}$

tau (as correlations)

INTRCPT1,B0 $1.000-0.207$

QUAD_TIM,B2 -0.207 1.000

Random level-1 coefficient Reliability estimate

$\begin{array}{lc}\text { INTRCPT1, G0 } & 0.926 \\ \text { QUAD_TIM, G2 } & 0.268\end{array}$

The value of the log-likelihood function at iteration $14=5.703277 \mathrm{E}+001$

Final estimation of fixed effects:

\begin{tabular}{|c|c|c|c|c|c|}
\hline Fixed Effect & $\begin{array}{c}\text { Standard } \\
\text { Coefficient Error }\end{array}$ & $\begin{array}{r}\text { Approx. } \\
\text { T-ratio }\end{array}$ & d.f. & P-value & \\
\hline \multicolumn{6}{|c|}{ For INTRCPT1, B0 } \\
\hline $\begin{array}{l}\text { For TIME slop } \\
\text { INTRCPT2, G1 }\end{array}$ & , B1 & 0.005269 & -0.036 & 183 & 0.972 \\
\hline \multicolumn{6}{|c|}{ For QUAD_TIM slope, B2 } \\
\hline INTRCPT 2, G2 & $0.014040 \quad 0$ & 0.004294 & 3.270 & 45 & 0.002 \\
\hline
\end{tabular}

Final estimation of variance components:

Random Effect Standard Variance df Chi-square P-value
Deviation Component

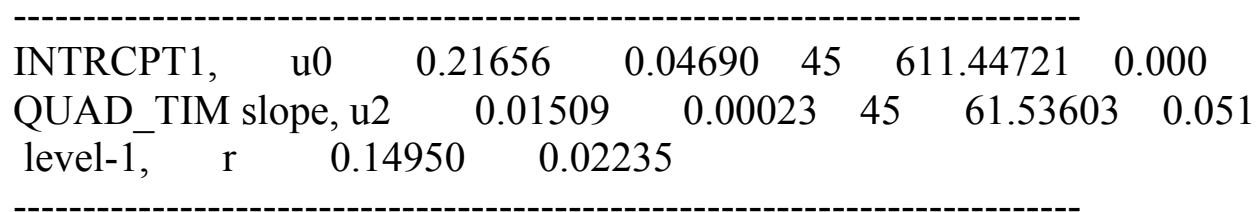

Statistics for current covariance components model

Deviance $\quad=-114.065549$

Number of estimated parameters $=4$

Variance-Covariance components test

Chi-square statistic $=18.69304$ 
Degrees of freedom $=1$

P-value $\quad=\quad<0.001$ 


\section{Appendix 4.4}

Model Equations and Results: Maternal-Reported Internalizing in the Competition Challenge

\section{Model 1}

Level-1 Model

LG_CORT $=\mathrm{B} 0+\mathrm{r}$

Level-2 Model

$$
\mathrm{B} 0=\mathrm{G} 00+\mathrm{u} 0
$$

Mixed Model

$\mathrm{LG} \_\mathrm{CORT}=\mathrm{G} 00+\mathrm{u} 0+\mathrm{r}$

$\operatorname{sigma}^{\wedge} 2=0.02518$

Standard error of $\operatorname{sigma}^{\wedge} 2=0.00235$

tau

INTRCPT1,B0 $\quad 0.04528$

Standard error of tau

INTRCPT1,B0 0.01032

Random level-1 coefficient Reliability estimate

INTRCPT1, G0 0.915

The value of the log-likelihood function at iteration $2=5.970427 \mathrm{E}+001$

The outcome variable is LG_CORT

Final estimation of fixed effects:

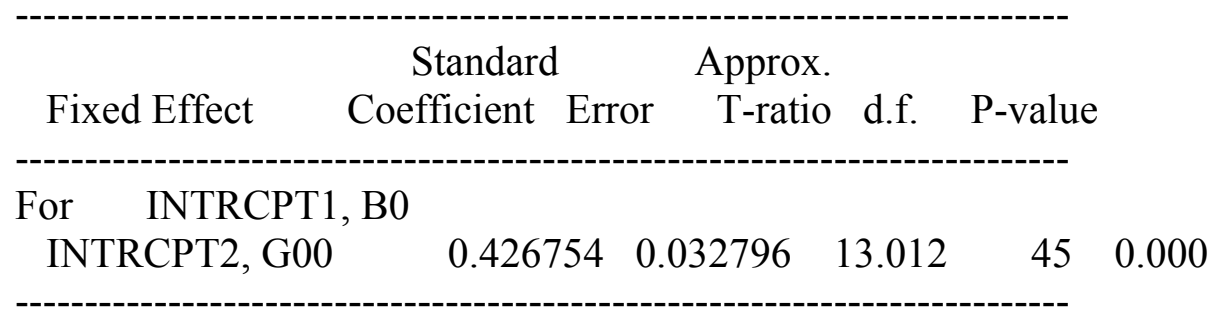

Final estimation of variance components: 

Random Effect Standard Variance df Chi-square P-value
Deviation Component

$\begin{array}{lllllll}\text { INTRCPT1, u0 } & 0.21279 & 0.04528 & 45 & 542.32638 & 0.000\end{array}$
$\begin{array}{llll}\text { level-1, } & \mathrm{r} & 0.15868 & 0.02518\end{array}$

Statistics for the current model

Deviance $\quad=-119.408532$

Number of estimated parameters $=3$

\section{Model 2}

Level-1 Model

$$
\text { LG_CORT }=\mathrm{B} 0+\mathrm{B} 1 *(\mathrm{TIME})+\mathrm{r}
$$

Level-2 Model

$$
\begin{aligned}
& \mathrm{B} 0=\mathrm{G} 00+\mathrm{u} 0 \\
& \mathrm{~B} 1=\mathrm{G} 10
\end{aligned}
$$

$$
\begin{aligned}
& \text { Mixed Model } \\
& \text { LG_CORT }=\text { G00 } \\
& + \text { G10*TIME }+\mathrm{u} 0+\mathrm{r}
\end{aligned}
$$

$\operatorname{sigma}^{\wedge} 2=0.02518$

Standard error of $\operatorname{sigma}^{\wedge} 2=0.00235$

tau

INTRCPT1,B0 0.04528

Standard error of tau

INTRCPT1,B0 0.01032

Random level-1 coefficient Reliability estimate

INTRCPT1, G0 $\quad 0.915$

The value of the log-likelihood function at iteration $2=5.970483 \mathrm{E}+001$

The outcome variable is LG_CORT 
Final estimation of fixed effects:

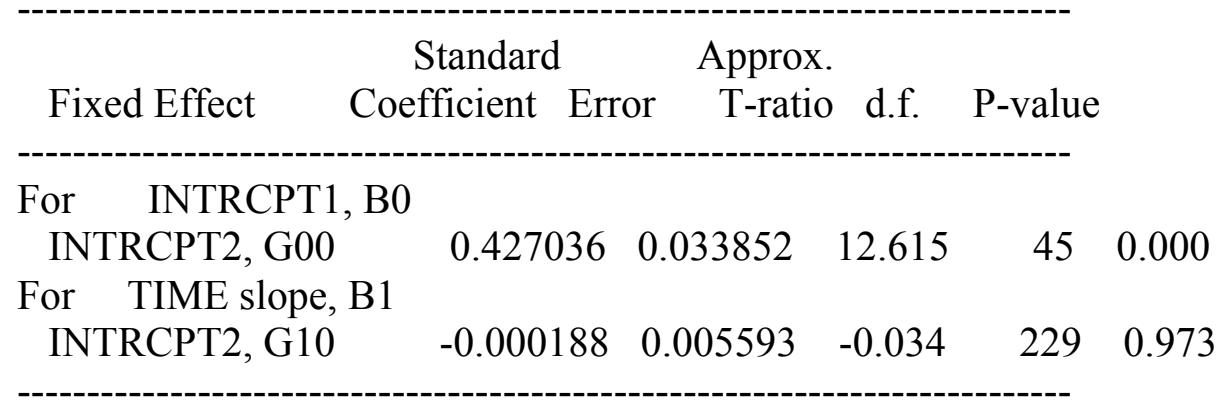

Final estimation of variance components:

Random Effect Standard Variance df Chi-square P-value Deviation Component

\begin{tabular}{|c|c|c|c|}
\hline INTRCPT1, & 0.21279 & $0.04528 \quad 45$ & 542.32904 \\
\hline level-1, $\quad r$ & $0.15868 \quad 0$ & 2518 & \\
\hline
\end{tabular}

Statistics for the current model

Deviance $\quad=-119.409659$

Number of estimated parameters $=4$

Model comparison test

$\begin{array}{ll}\text { Chi-square statistic } & =0.00113 \\ \text { Degrees of freedom } & =1 \\ \text { P-value }= & >.500\end{array}$

\section{Model 3}

Level-1 Model

$$
\text { LG_CORT }=\text { B0 }+ \text { B } 1 *(\text { TIME })+\text { B2 } *\left(Q U A D \_T I M\right)+r
$$

Level-2 Model

$$
\begin{aligned}
& \mathrm{B} 0=\mathrm{G} 00+\mathrm{u} 0 \\
& \mathrm{~B} 1=\mathrm{G} 10 \\
& \mathrm{~B} 2=\mathrm{G} 20
\end{aligned}
$$

Mixed Model

LG_CORT $=$ G00 
+ G10*TIME

$+\mathrm{G} 20 * \mathrm{QUAD}$ TIM $+\mathrm{u} 0+\mathrm{r}$

$\operatorname{sigma}^{\wedge} 2=0.02376$

Standard error of $\operatorname{sigma}^{\wedge} 2=0.00222$

tau

INTRCPT1,B0 0.04552

Standard error of tau

INTRCPT1,B0 0.01032

Random level-1 coefficient Reliability estimate

INTRCPT1, G0 $\quad 0.920$

The value of the log-likelihood function at iteration $2=6.637930 \mathrm{E}+001$

The outcome variable is LG_CORT

Final estimation of fixed effects:

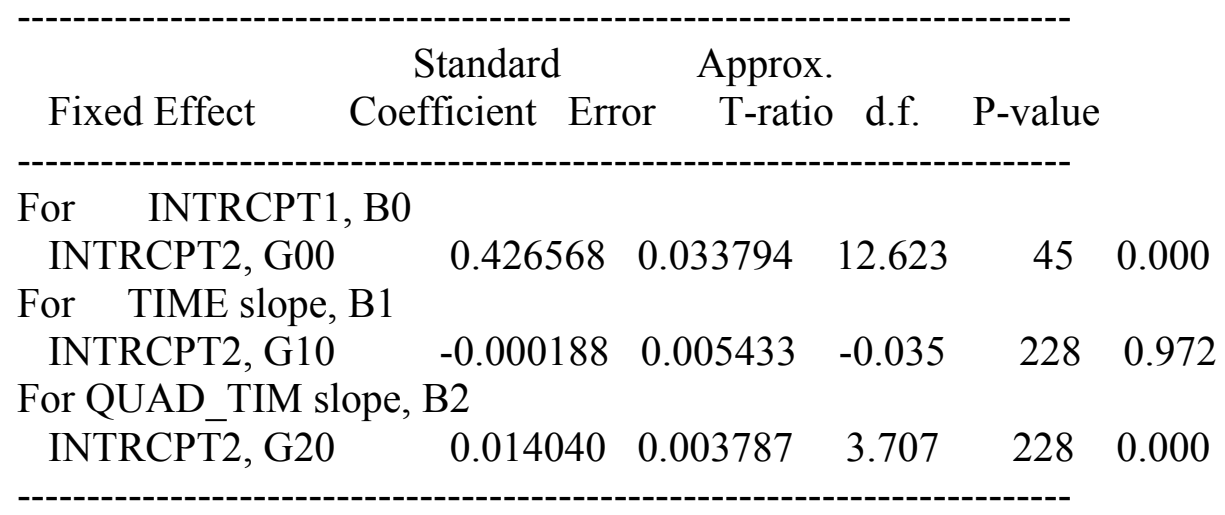

Final estimation of variance components:

Random Effect Standard Variance df Chi-square P-value Deviation Component

\begin{tabular}{|c|c|c|c|c|}
\hline INTRCPT1, & 0.21335 & 0.04552 & 45 & 574.73653 \\
\hline level-1, $\quad \mathrm{r}$ & 0.15414 & 2376 & & \\
\hline
\end{tabular}

Statistics for the current model 
Deviance $\quad=-132.758591$

Number of estimated parameters $=5$

Model comparison test

Chi-square statistic $=13.34893$

Degrees of freedom $=1$

P-value $\quad=\quad<0.001$

\section{Model 4}

Level-1 Model

$$
\text { LG_CORT }=\mathrm{B} 0+\mathrm{B} 1 *(\mathrm{TIME})+\mathrm{B} 2 *\left(\mathrm{QUAD} \_\mathrm{TIM}\right)+\mathrm{B} 3 *\left(\mathrm{LG} \_\mathrm{SAA}\right)+\mathrm{r}
$$

Level-2 Model

$$
\begin{aligned}
& \mathrm{B} 0=\mathrm{G} 00+\mathrm{u} 0 \\
& \mathrm{~B} 1=\mathrm{G} 10 \\
& \mathrm{~B} 2=\mathrm{G} 20 \\
& \mathrm{~B} 3=\mathrm{G} 30
\end{aligned}
$$

LG_SAA has been centered around the group mean.

$$
\begin{aligned}
& \text { Mixed Model } \\
& \text { LG_CORT = G00 } \\
& + \text { G10*TIME } \\
& + \text { G20*QUAD_TIM } \\
& + \text { G30*LG_SAA } \\
& +\mathrm{u} 0+\mathrm{r}
\end{aligned}
$$

$\operatorname{sigma}^{\wedge} 2=0.02400$

Standard error of $\operatorname{sigma}^{\wedge} 2=\quad 0.00263$

tau

INTRCPT1,B0 0.03355

Standard error of tau

INTRCPT1,B0 0.00839

Random level-1 coefficient Reliability estimate

INTRCPT1, G0 0.874


The value of the log-likelihood function at iteration $4=5.055731 \mathrm{E}+001$

The outcome variable is LG_CORT

Final estimation of fixed effects:

\begin{tabular}{|c|c|c|c|c|c|}
\hline Fixed Effect & $\begin{array}{c}\text { Standard } \\
\text { Coefficient Err }\end{array}$ & $\begin{array}{r}\text { Approx. } \\
\text { or } \quad \text { T-ratio }\end{array}$ & d.f. & P-value & \\
\hline \multicolumn{6}{|c|}{ For INTRCPT1, B0 } \\
\hline INTRCPT2, G00 & 0.447353 & 0.031298 & 14.293 & 41 & 0.000 \\
\hline \multicolumn{6}{|c|}{ For TIME slope, B1 } \\
\hline INTRCPT2, G10 & -0.003697 & 0.009949 & -0.372 & 164 & 0.71 \\
\hline \multicolumn{6}{|c|}{ For QUAD_TIM slope, B2 } \\
\hline INTRCPT2, G20 & 0.012138 & 0.006513 & 1.864 & 164 & 0.064 \\
\hline \multicolumn{6}{|c|}{ For_LG_SAA slope, B3 } \\
\hline INTRCי-PT2, G30 & 0.069475 & 0.104917 & 0.662 & 164 & 0.509 \\
\hline
\end{tabular}

Final estimation of variance components:

Random Effect Standard Variance df Chi-square P-value Deviation Component

$\begin{array}{lllllll}\text { INTRCPT1, u0 } & 0.18317 & 0.03355 & 41 & 335.27577 & 0.000\end{array}$
$\begin{array}{llll}\text { level-1, } & \text { r } & 0.15493 & 0.02400\end{array}$

Statistics for the current model

Deviance $\quad=-101.114613$

Number of estimated parameters $=6$

Model comparison test

Chi-square statistic $=31.64398$

Degrees of freedom $=1$

P-value $\quad=\quad<0.001$

\section{Model 5}

Level-1 Model

$$
\text { LG_CORT }=\text { B0 }+ \text { B } 1 *(T I M E)+B 2 *\left(Q U A D \_T I M\right)+B 3 *\left(L G \_S A A\right)+B 4 *\left(S A A X Q \_T I\right)+r
$$

Level-2 Model

$$
\mathrm{B} 0=\mathrm{G} 00+\mathrm{u} 0
$$




$$
\begin{aligned}
& \mathrm{B} 1=\mathrm{G} 10 \\
& \mathrm{~B} 2=\mathrm{G} 20 \\
& \mathrm{~B} 3=\mathrm{G} 30 \\
& \mathrm{~B} 4=\mathrm{G} 40
\end{aligned}
$$

LG_SAA has been centered around the group mean.

$$
\begin{aligned}
& \text { Mixed Model } \\
& \text { LG_CORT = G00 } \\
& + \text { G10*TIME } \\
& + \text { G20*QUAD_TIM } \\
& \text { + G30*LG_SAA } \\
& \text { + G40*SAAXQ_TI } \\
& +\mathrm{u} 0+\mathrm{r}
\end{aligned}
$$

$\operatorname{sigma}^{\wedge} 2=0.02402$

\begin{tabular}{|c|c|c|c|c|c|}
\hline Fixed Effect & $\begin{array}{c}\text { Standard } \\
\text { Coefficient Err }\end{array}$ & $\begin{array}{r}\text { Approx. } \\
\text { or } \quad \text { T-ratio }\end{array}$ & d.f. & P-value & \\
\hline \multicolumn{6}{|c|}{ For INTRCPT1, B0 } \\
\hline INTRCPT2, G00 & 0.447863 & 0.031295 & 14.311 & 41 & 0.000 \\
\hline \multicolumn{6}{|c|}{ For TIME slope, B1 } \\
\hline INTRCPT2, G10 & -0.003733 & 0.009953 & -0.375 & 163 & 0.70 \\
\hline \multicolumn{6}{|c|}{ For QUAD_TIM slope, B2 } \\
\hline INTRCPT'2, G20 & 0.012357 & 0.006568 & 1.881 & 163 & 0.062 \\
\hline
\end{tabular}

Standard error of $\operatorname{sigma}^{\wedge} 2=0.00263$

tau

INTRCPT1,B0 0.03338

Standard error of tau

INTRCPT1,B0 0.00835

Random level-1 coefficient Reliability estimate

INTRCPT1, G0 $\quad 0.874$

The value of the log-likelihood function at iteration $7=5.059164 \mathrm{E}+001$

The outcome variable is LG_CORT

Final estimation of fixed effects: 
For LG_SAA slope, B3

$\begin{array}{llllll}\text { INTRCPT2, G30 } & 0.066055 & 0.105757 & 0.625 & 163 & 0.533\end{array}$

For SAAXQ TI slope, B4

$\begin{array}{llllll}\text { INTRCPT2, G40 } & 0.010246 & 0.038970 & 0.263 & 163 & 0.793\end{array}$

Final estimation of variance components:

Random Effect Standard Variance df Chi-square P-value Deviation Component

INTRCPT1, u0 $0.18269 \quad 0.03338 \quad 41 \quad 333.54901 \quad 0.000$

level-1, $\quad \mathrm{r} \quad 0.15499 \quad 0.02402$

Statistics for the current model

Deviance $\quad=-101.183276$

Number of estimated parameters $=7$

Model comparison test

Chi-square statistic $=0.06866$

Degrees of freedom $=1$

P-value $\quad=\quad>.500$

\section{Model 6}

Level-1 Model

$$
\text { LG_CORT }=\mathrm{B} 0+\mathrm{B} 1 *(\mathrm{TIME})+\mathrm{B} 2 *\left(\mathrm{QUAD} \_\mathrm{TIM}\right)+\mathrm{B} 3 *\left(\mathrm{LG} \_\mathrm{SAA}\right)+\mathrm{B} 4 *(\mathrm{SAAXTIME})+\mathrm{r}
$$

Level-2 Model

$$
\begin{aligned}
& \mathrm{B} 0=\mathrm{G} 00+\mathrm{u} 0 \\
& \mathrm{~B} 1=\mathrm{G} 10 \\
& \mathrm{~B} 2=\mathrm{G} 20 \\
& \mathrm{~B} 3=\mathrm{G} 30 \\
& \mathrm{~B} 4=\mathrm{G} 40
\end{aligned}
$$

LG_SAA has been centered around the group mean.

$$
\begin{aligned}
& \text { Mixed Model } \\
& \text { LG_CORT }=\text { G00 } \\
& + \text { G10*TIME } \\
& + \text { G20*QUAD_TIM }
\end{aligned}
$$


+ G30*LG_SAA

+ G40*SA $\bar{A}$ XTIME

$+\mathrm{u} 0+\mathrm{r}$

$\operatorname{sigma}^{\wedge} 2=0.02401$

Standard error of $\operatorname{sigma}^{\wedge} 2=0.00263$

tau

INTRCPT1,B0 0.03292

Standard error of tau

INTRCPT1,B0 0.00826

Random level-1 coefficient Reliability estimate

INTRCPT1, G0 $\quad 0.872$

The value of the log-likelihood function at iteration $7=5.087204 \mathrm{E}+001$

The outcome variable is LG_CORT

Final estimation of fixed effects:

\begin{tabular}{|c|c|c|c|c|c|}
\hline Fixed Effect & $\begin{array}{c}\text { Standard } \\
\text { Coefficient Erro }\end{array}$ & $\begin{array}{r}\text { Approx. } \\
\text { or } \quad \text { T-ratio }\end{array}$ & d.f. & P-value & \\
\hline \multicolumn{6}{|c|}{ For INTRCPT1, B0 } \\
\hline \multicolumn{6}{|c|}{ For TIME slope, B1 } \\
\hline INTRCPT2, G1C & -0.003568 & 0.009952 & -0.358 & 163 & 0.720 \\
\hline \multicolumn{6}{|c|}{ For QUAD_TIM slope, B2 } \\
\hline INTRCPT2, G20 & 0.012747 & 0.006559 & 1.943 & 163 & 0.054 \\
\hline \multicolumn{6}{|c|}{ For LG_SAA slope, B3 } \\
\hline INTRCPT2, G3C & 0.021272 & 0.121117 & 0.176 & 163 & 0.861 \\
\hline \multicolumn{6}{|c|}{ For SAAXTIME slope, B4 } \\
\hline INTRCPT2, G4C & -0.042742 & 0.053627 & -0.797 & 163 & 0.427 \\
\hline
\end{tabular}

Final estimation of variance components:

Random Effect Standard Variance df Chi-square P-value Deviation Component

$\begin{array}{lllllll}\text { INTRCPT1, } & \text { u0 } & 0.18144 & 0.03292 & 41 & 329.66441 & 0.000\end{array}$ 
$\begin{array}{llll}\text { level-1, } & \mathrm{r} & 0.15496 & 0.02401\end{array}$

Statistics for the current model

Deviance $\quad=-101.744088$

Number of estimated parameters $=7$

Model comparison test

Chi-square statistic $=0.62948$

Degrees of freedom $=1$

$\mathrm{P}$-value $\quad=\quad>.500$

\section{Model 7}

Level-1 Model

LG_CORT $=\mathrm{B} 0+\mathrm{B} 1 *(\mathrm{TIME})+\mathrm{B} 2 *\left(\mathrm{QUAD} \_\mathrm{TIM}\right)+\mathrm{B} 3 *\left(\mathrm{LG}_{-} \mathrm{SAA}\right)+\mathrm{B} 4 *(\mathrm{SAAXTIME})+$ $\mathrm{B} 5 *(\mathrm{SAAX} \overline{\mathrm{X}} \mathrm{TI})+\mathrm{r}$

Level-2 Model

$$
\begin{aligned}
& \mathrm{B} 0=\mathrm{G} 00+\mathrm{u} 0 \\
& \mathrm{~B} 1=\mathrm{G} 10 \\
& \mathrm{~B} 2=\mathrm{G} 20 \\
& \mathrm{~B} 3=\mathrm{G} 30 \\
& \mathrm{~B} 4=\mathrm{G} 40 \\
& \mathrm{~B} 5=\mathrm{G} 50
\end{aligned}
$$

LG_SAA has been centered around the group mean.

$$
\begin{aligned}
& \text { Mixed Model } \\
& \text { LG_CORT }=\text { G00 } \\
& + \text { G10*TIME } \\
& + \text { G20*QUAD_TIM } \\
& \text { + G30*LG_SAA } \\
& \text { + G40*SAAXTIME } \\
& \text { + G50*SAAXQ_TI } \\
& \text { + u0+ r }
\end{aligned}
$$

$\operatorname{sigma}^{\wedge} 2=0.02399$

Standard error of $\operatorname{sigma}^{\wedge} 2=0.00263$

tau 
INTRCPT1,B0 0.03298

Standard error of tau

INTRCPT1,B0 0.00827

Random level-1 coefficient Reliability estimate

INTRCPT1, G0 $\quad 0.872$

The value of the log-likelihood function at iteration $7=5.092107 \mathrm{E}+001$

The outcome variable is LG_CORT

Final estimation of fixed effects:

\begin{tabular}{|c|c|c|c|c|c|}
\hline Fixed Effect & $\begin{array}{c}\text { Standard } \\
\text { Coefficient Erro }\end{array}$ & $\begin{array}{r}\text { Approx. } \\
\text { or } \quad \text { T-ratio }\end{array}$ & d.f. & P-value & \\
\hline \multicolumn{6}{|c|}{ For $\quad$ INTRCPT1, B0 } \\
\hline INTRCPT2, G00 & 0.448250 & 0.031144 & 14.393 & 41 & 0.000 \\
\hline \multicolumn{6}{|c|}{ For TIME slope, B1 } \\
\hline INTRCPT2, G10 & -0.003470 & 0.009952 & -0.349 & 162 & 0.728 \\
\hline \multicolumn{6}{|c|}{ For QUAD_TIM slope, B2 } \\
\hline INTRCPT2, G20 & 0.012606 & 0.006571 & 1.918 & 162 & 0.057 \\
\hline \multicolumn{6}{|c|}{ For LG_SAA slope, B3 } \\
\hline INTRCPT2, G30 & 0.010942 & 0.125522 & 0.087 & 162 & 0.931 \\
\hline \multicolumn{6}{|c|}{ For SAAXTIME slope, B4 } \\
\hline INTRCPT2, G40 & -0.056589 & 0.069586 & -0.813 & 162 & 0.417 \\
\hline \multicolumn{6}{|c|}{ For SAAXQ_TI slope, B5 } \\
\hline INTRCPT2, G50 & -0.015838 & 0.050548 & -0.313 & 162 & 0.75 \\
\hline
\end{tabular}

Final estimation of variance components:

Random Effect Standard Variance df Chi-square P-value Deviation Component

$\begin{array}{llllll}\text { INTRCPT1, u0 } & 0.18161 & 0.03298 & 41 & 330.48039 & 0.000\end{array}$
$\begin{array}{llll}\text { level-1, } & \text { r } & 0.15488 & 0.02399\end{array}$

Statistics for the current model

Deviance $\quad=-101.842139$ 
Number of estimated parameters $=8$

Model comparison test

Chi-square statistic $=0.09805$

Degrees of freedom $=1$

P-value $\quad=\quad>.500$

\section{Model 7.1}

Level-1 Model

LG_CORT $=$ B0 + B $1 *(T I M E)+B 2 *\left(Q U A D \_T I M\right)+B 3 *\left(L G \_S A A\right)+B 4 *($ SAAXTIME $)+$ $\mathrm{B} 5 *(\mathrm{SAAXQ} \mathrm{TI})+\mathrm{r}$

Level-2 Model

$$
\begin{aligned}
& \mathrm{B} 0=\mathrm{G} 00+\mathrm{u} 0 \\
& \mathrm{~B} 1=\mathrm{G} 10+\mathrm{u} 1 \\
& \mathrm{~B} 2=\mathrm{G} 20 \\
& \mathrm{~B} 3=\mathrm{G} 30 \\
& \mathrm{~B} 4=\mathrm{G} 40 \\
& \mathrm{~B} 5=\mathrm{G} 50
\end{aligned}
$$

LG_SAA has been centered around the group mean.

$$
\begin{aligned}
& \text { Mixed Model } \\
& \text { LG_CORT }=\text { G00 } \\
& \text { + G10*TIME } \\
& \text { + G20*QUAD_TIM } \\
& \text { + G30*LG_SAA } \\
& \text { + G40*SAAXTIME } \\
& \text { + G50*SAAXQ_TI } \\
& \text { + u0 + u1*TIME + r }
\end{aligned}
$$

$\operatorname{sigma}^{\wedge} 2=0.02128$

Standard error of sigma^ ${ }^{\wedge}=0.00269$

tau

$\begin{array}{lll}\text { INTRCPT1,B0 } & 0.02679 & 0.00284\end{array}$

$\begin{array}{lll}\text { TIME,B } 1 \quad 0.00284 & 0.00109\end{array}$

Standard errors of tau

$\begin{array}{lll}\text { INTRCPT1,B0 } & 0.00730 & 0.00163\end{array}$

TIME,B1 $0.00163 \quad 0.00076$ 
tau (as correlations)

INTRCPT1,B0 $1.000 \quad 0.526$

TIME,B1 0.5261 .000

Random level-1 coefficient Reliability estimate

$\begin{array}{lc}\text { INTRCPT1, G0 } & 0.804 \\ \text { TIME, G1 } & 0.335\end{array}$

The value of the log-likelihood function at iteration $18=5.512945 \mathrm{E}+001$

The outcome variable is LG_CORT

Final estimation of fixed effects:

\begin{tabular}{|c|c|c|c|c|c|}
\hline Fixed Effect & $\begin{array}{c}\text { Standard } \\
\text { Coefficient Erro }\end{array}$ & $\begin{array}{r}\text { Approx. } \\
\text { T-ratio }\end{array}$ & d.f. & P-value & \\
\hline \multicolumn{6}{|c|}{ For INTRCPT1, B0 } \\
\hline \multicolumn{6}{|c|}{ For TIME slope, B1 } \\
\hline INTRCPT2, G1 & -0.003913 & 0.010671 & -0.367 & 41 & 0.716 \\
\hline \multicolumn{6}{|c|}{ For QUAD_TIM slope, B2 } \\
\hline $\begin{array}{l}\text { INTRCPT } 2, \text { G } 2 \\
\text { For LG SAA sl }\end{array}$ & ee. B3 0.012734 & 0.006 & 054 & 121 & 0.042 \\
\hline INTRCPT2, G3 & -0.019857 & 0.124469 & -0.160 & 121 & 0.874 \\
\hline \multicolumn{6}{|c|}{ For SAAXTIME slope, B4 } \\
\hline INTRCPT2, G4 & -0.051997 & 0.072871 & -0.714 & 121 & 0.477 \\
\hline \multicolumn{6}{|c|}{ For SAAXQ_TI slope, B5 } \\
\hline INTRCPT2, G5 & -0.013151 & 0.048713 & -0.270 & 121 & 0.788 \\
\hline
\end{tabular}

Final estimation of variance components:

Random Effect Standard Variance df Chi-square P-value Deviation Component

\begin{tabular}{ccccccc}
\hline INTRCPT1, u0 & 0.16366 & 0.02679 & 41 & 218.48118 & 0.000 \\
TIME slope, u1 & 0.03295 & 0.00109 & 41 & 63.83974 & 0.013 \\
level-1, r & 0.14587 & 0.02128 & & &
\end{tabular}

Statistics for the current model 
Deviance $\quad=-110.258891$

Number of estimated parameters $=10$

Model comparison test

Chi-square statistic $=8.41675$

Degrees of freedom $=2$

P-value $\quad=0.015$

\section{Model 7.2}

Level-1 Model

LG_CORT $=$ B $0+$ B $1 *($ TIME $)+$ B2 $*($ QUAD_TIM $)+$ B $3 *\left(L G \_S A A\right)+B 4 *($ SAAXTIME $)+$ $\mathrm{B} 5 *\left(\mathrm{SAAXQ}{ }_{-} \mathrm{TI}\right)+\mathrm{r}$

Level-2 Model

$$
\begin{aligned}
& \mathrm{B} 0=\mathrm{G} 00+\mathrm{u} 0 \\
& \mathrm{~B} 1=\mathrm{G} 10 \\
& \mathrm{~B} 2=\mathrm{G} 20+\mathrm{u} 2 \\
& \mathrm{~B} 3=\mathrm{G} 30 \\
& \mathrm{~B} 4=\mathrm{G} 40 \\
& \mathrm{~B} 5=\mathrm{G} 50
\end{aligned}
$$

LG_SAA has been centered around the group mean.

$$
\begin{aligned}
& \text { Mixed Model } \\
& \text { LG_CORT = G00 } \\
& \text { + G10*TIME } \\
& \text { + G20*QUAD_TIM } \\
& \text { + G30*LG_SAA } \\
& \text { + G40*SAAXTIME } \\
& \text { + G50*SAAXQ_TI } \\
& \text { + u0 + u2*QUAD_TIM + r }
\end{aligned}
$$

$\operatorname{sigma}^{\wedge} 2=0.01932$

Standard error of sigma ${ }^{\wedge} 2=0.00244$

tau

$$
\begin{array}{lll}
\text { INTRCPT1,B0 } & 0.03054 & -0.00250 \\
\text { QUAD_TIM,B2 } & -0.00250 & 0.00081
\end{array}
$$

Standard errors of tau

$\begin{array}{lll}\text { INTRCPT1,B0 } & 0.00761 & 0.00121\end{array}$

$\begin{array}{lll}\text { QUAD_TIM,B2 } & 0.00121 & 0.00038\end{array}$ 
tau (as correlations)

INTRCPT1,B0 $1.000-0.502$

QUAD_TIM,B2 -0.502 1.000

Random level-1 coefficient Reliability estimate

INTRCPT1, G0 $\quad 0.874$

QUAD_TIM, G2 $\quad 0.485$

The value of the log-likelihood function at iteration $13=5.810132 \mathrm{E}+001$ 
The outcome variable is LG_CORT

Final estimation of fixed effects:

\begin{tabular}{|c|c|c|c|c|c|}
\hline Fixed Effect & $\begin{array}{c}\text { Standard } \\
\text { Coefficient Erro }\end{array}$ & $\begin{array}{rr} & \text { Approx. } \\
\text { or } & \text { T-ratio }\end{array}$ & d.f. & P-value & \\
\hline \multicolumn{6}{|c|}{ For INTRCPT1, B0 } \\
\hline INTRCPT2, G00 & 0.448835 & 0.029635 & 15.145 & 41 & 0.000 \\
\hline \multicolumn{6}{|c|}{ For TIME slope, B1 } \\
\hline INTRCPT2, G10 & -0.004024 & 0.008943 & -0.450 & 121 & 0.654 \\
\hline \multicolumn{6}{|c|}{ For QUAD TIM slope, B2 } \\
\hline INTRCPT2, G20 & 0.012700 & 0.007379 & 1.721 & 41 & 0.093 \\
\hline \multicolumn{6}{|c|}{ For LG_SAA slope, B3 } \\
\hline INTRCPT2, G30 & -0.044759 & 0.122530 & -0.365 & 121 & 0.716 \\
\hline \multicolumn{6}{|c|}{ For SAAXTIME slope, B4 } \\
\hline INTRCPT2, G40 & -0.062670 & 0.063017 & -0.994 & 121 & 0.322 \\
\hline \multicolumn{6}{|c|}{ For SAAXQ TI slope, B5 } \\
\hline INTRCPT2, G50 & -0.022977 & 0.049630 & -0.463 & 121 & 0.644 \\
\hline
\end{tabular}

Final estimation of variance components:

Random Effect Standard Variance df Chi-square P-value Deviation Component

$\begin{array}{lllllll} & \text { INTRCPT1, u0 } & 0.17476 & 0.03054 & 41 & 348.15708 & 0.000 \\ \text { QUAD_TIM slope, u2 } & 0.02848 & 0.00081 & 41 & 82.19075 & 0.000 \\ \text { level-1, } \quad \mathrm{r} & 0.13901 & 0.01932 & & & & \end{array}$

Statistics for the current model

Deviance $\quad=-116.202640$

Number of estimated parameters $=10$

Model comparison test

Chi-square statistic $=14.36050$
Degrees of freedom $=2$
P-value $=0.001$

\section{Model 7.3}

Level-1 Model 
LG_CORT $=$ B0 + B1*(TIME $)+B 2 *\left(Q U A D \_T I M\right)+B 3 *\left(L G \_S A A\right)+B 4 *(S A A X T I M E)+$ $\mathrm{B} 5 *\left(\mathrm{SAAX} \mathrm{Q}_{-} \mathrm{TI}\right)+\mathrm{r}$

Level-2 Model

$$
\begin{aligned}
& \mathrm{B} 0=\mathrm{G} 00+\mathrm{u} 0 \\
& \mathrm{~B} 1=\mathrm{G} 10 \\
& \mathrm{~B} 2=\mathrm{G} 20 \\
& \mathrm{~B} 3=\mathrm{G} 30+\mathrm{u} 3 \\
& \mathrm{~B} 4=\mathrm{G} 40 \\
& \mathrm{~B} 5=\mathrm{G} 50
\end{aligned}
$$

LG_SAA has been centered around the group mean.

$$
\begin{aligned}
& \text { Mixed Model } \\
& \text { LG_CORT }=\text { G00 } \\
& \text { + G10*TIME } \\
& \text { + G20*QUAD_TIM } \\
& \text { + G30*LG_SAA } \\
& \text { + G40*SAAXTIME } \\
& \text { + G50*SAAXQ_TI } \\
& \text { + u0 + u3*LG_SAA + r }
\end{aligned}
$$

$\operatorname{sigma}^{\wedge} 2=0.02142$

Standard error of $\operatorname{sigma}^{\wedge} 2=0.00257$

$$
\begin{array}{lcc}
\text { tau } & & \\
\text { INTRCPT1,B0 } & 0.03410 & 0.01807 \\
\text { LG_SAA,B3 } & 0.01807 & 0.18271
\end{array}
$$

Standard errors of tau

$$
\begin{array}{ccc}
\text { INTRCPT1,B0 } & 0.00840 & 0.02546 \\
\text { LG_SAA,B3 } & 0.02546 & 0.12942
\end{array}
$$

tau (as correlations)

INTRCPT1,B0 $1.000 \quad 0.229$

LG_SAA,B3 0.2291 .000

Random level-1 coefficient Reliability estimate

$\begin{array}{cc}\text { INTRCPT1, G0 } & 0.888 \\ \text { LG_SAA, G3 } & 0.259\end{array}$

The value of the log-likelihood function at iteration $13=5.325547 \mathrm{E}+001$ 
The outcome variable is LG_CORT

Final estimation of fixed effects:

\begin{tabular}{|c|c|c|c|c|c|}
\hline Fixed Effect & $\begin{array}{c}\text { Standard } \\
\text { Coefficient Err }\end{array}$ & $\begin{array}{l}\text { Approx } \\
\text { T-ratic }\end{array}$ & d.f. & P-value & \\
\hline \multicolumn{6}{|c|}{ For INTRCPT1, B0 } \\
\hline INTRCPT2, G00 & 0.447252 & 0.031322 & 14.279 & 41 & 0.000 \\
\hline \multicolumn{6}{|c|}{ For TIME slope, B1 } \\
\hline INTRCPT2, G10 & -0.002080 & 0.009676 & -0.215 & 121 & 0.830 \\
\hline \multicolumn{6}{|c|}{ For QUAD_TIM slope, B2 } \\
\hline INTRCPT2, G20 & 0.014770 & 0.006349 & 2.326 & 121 & 0.022 \\
\hline \multicolumn{6}{|c|}{ For LG_SAA slope, B3 } \\
\hline INTRCPPT2, G30 & 0.088260 & 0.145581 & 0.606 & 41 & 0.548 \\
\hline \multicolumn{6}{|c|}{ For SAAXTIME slope, B4 } \\
\hline INTRCPT2, G40 & -0.017040 & 0.068470 & -0.249 & 121 & 0.804 \\
\hline \multicolumn{6}{|c|}{ For SAAXQ_TI slope, B5 } \\
\hline INTRCPT2, G50 & -0.017504 & 0.049914 & -0.351 & 121 & 0.726 \\
\hline
\end{tabular}

Final estimation of variance components:

Random Effect Standard Variance df Chi-square P-value Deviation Component

$\begin{array}{llccccc}\text { INTRCPT1, u0 } & 0.18466 & 0.03410 & 41 & 376.10762 & 0.000 \\ \text { LG_SAA slope, } \mathrm{u} 3 & 0.42744 & 0.18271 & 41 & 50.97844 & 0.137 \\ \text { level-1, } & \mathrm{r} & 0.14635 & 0.02142 & & & \end{array}$

Statistics for the current model

Deviance $\quad=-106.510940$

Number of estimated parameters $=10$

Model comparison test

Chi-square statistic $=4.66880$

Degrees of freedom $=2$

$\mathrm{P}$-value $\quad 0.095$

\section{Model 7.4}

Level-1 Model 
LG_CORT $=$ B0 + B $1 *(T I M E)+B 2 *\left(Q U A D \_T I M\right)+B 3 *\left(L G \_S A A\right)+B 4 *(S A A X T I M E)+$ $\mathrm{B} 5 *(\mathrm{SAAXQ} \mathrm{TI})+\mathrm{r}$

Level-2 Model

$$
\begin{aligned}
& \mathrm{B} 0=\mathrm{G} 00+\mathrm{u} 0 \\
& \mathrm{~B} 1=\mathrm{G} 10+\mathrm{u} 1 \\
& \mathrm{~B} 2=\mathrm{G} 20+\mathrm{u} 2 \\
& \mathrm{~B} 3=\mathrm{G} 30 \\
& \mathrm{~B} 4=\mathrm{G} 40 \\
& \mathrm{~B} 5=\mathrm{G} 50
\end{aligned}
$$

LG_SAA has been centered around the group mean.

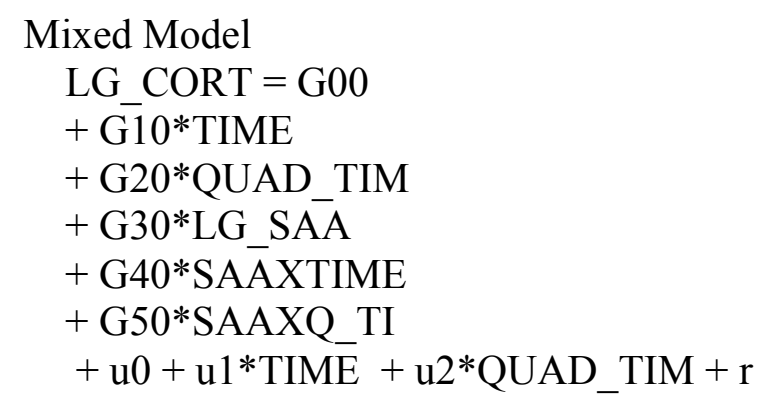

$\operatorname{sigma}^{\wedge} 2=0.01858$

Standard error of $\operatorname{sigma}^{\wedge} 2=\quad 0.00287$

tau

INTRCPT1,B0 $0.02869 \quad 0.00119 \quad-0.00151$

$\begin{array}{llll}\text { TIME,B } 1 & 0.00119 & 0.00030 & -0.00038\end{array}$

$\begin{array}{llll}\text { QUAD_TIM,B2 } & -0.00151 & -0.00038 & 0.00050\end{array}$

Standard errors of tau

$\begin{array}{llll}\text { INTRCPT1,B0 } & 0.00759 & 0.00174 & 0.00128\end{array}$

$\begin{array}{llll}\text { TIME,B } 1 & 0.00174 & 0.00090 & 0.00047\end{array}$

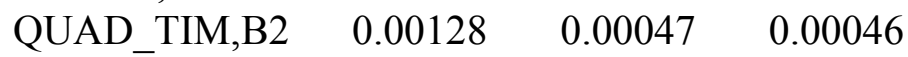

tau (as correlations)

INTRCPT1,B0 $1.000 \quad 0.408-0.399$

TIME,B1 $0.408 \quad 1.000-0.990$

QUAD_TIM,B2 -0.399-0.990 1.000

Random level-1 coefficient Reliability estimate 
INTRCPT1, G0

TIME, G1

QUAD_TIM, G2
0.826

0.086

0.264

The value of the log-likelihood function at iteration $5844=5.928108 \mathrm{E}+001$

The outcome variable is LG_CORT

Final estimation of fixed effects:

\begin{tabular}{|c|c|c|c|c|c|}
\hline Fixed Effect & $\begin{array}{c}\text { Standard } \\
\text { Coefficient Error }\end{array}$ & Approx. & d.f. & \multicolumn{2}{|l|}{ P-value } \\
\hline \multicolumn{6}{|c|}{ For INTRCPT1, B0 } \\
\hline INTRCPT2, G00 & 0.449907 & 0.028798 & 15.623 & 41 & 0.000 \\
\hline \multicolumn{6}{|c|}{ For TIME slope, B1 } \\
\hline INTRCPT2, G10 & -0.004279 & 0.009169 & -0.467 & 41 & 0.643 \\
\hline \multicolumn{6}{|c|}{ For QUAD_TIM slope, B2 } \\
\hline INTRCPT2, G20 & 0.013109 & 0.006770 & 1.936 & 41 & 0.060 \\
\hline \multicolumn{6}{|c|}{ For LG_SAA slope, B3 } \\
\hline INTRCPT2, G30 & -0.058451 & 0.124465 & -0.470 & 80 & 0.640 \\
\hline \multicolumn{6}{|c|}{ For SAAXTIME slope, B4 } \\
\hline INTRCPT2, G40 & -0.058670 & 0.063753 & -0.920 & 80 & 0.360 \\
\hline \multicolumn{6}{|c|}{ For SAAXQ_TI slope, B5 } \\
\hline INTRCPT2, G50 & -0.007257 & 0.048452 & -0.150 & 80 & 0.881 \\
\hline
\end{tabular}

The outcome variable is LG_CORT

Final estimation of variance components:

Random Effect Standard Variance df Chi-square P-value Deviation Component

$\begin{array}{lllllll}\text { INTRCPT1, u0 } & 0.16939 & 0.02869 & 41 & 250.16030 & 0.000 \\ \text { TIME slope, u1 } & 0.01725 & 0.00030 & 41 & 40.37455 & >.500 \\ \text { QUAD_TIM slope, u2 } & 0.02237 & 0.00050 & 41 & 52.37501 & 0.110 \\ \text { level-1, r } & 0.13631 & 0.01858 & & & & \end{array}$

Statistics for the current model

Deviance $\quad=-118.562156$

Number of estimated parameters $=13$

Model comparison test 
Chi-square statistic $=16.72002$

Degrees of freedom $=5$

P-value $\quad=0.005$

\section{Model 8}

Level-1 Model

LG_CORT $=$ B0 + B1 $*($ TIME $)+$ B2 $*\left(Q U A D \_T I M\right)+B 3 *\left(L G \_S A A\right)+B 4 *($ SAAXTIME $)+$ $\mathrm{B} 5 *(\mathrm{SAAXQ} \mathrm{Q}$ TI $)+\mathrm{r}$

Level-2 Model

$$
\begin{aligned}
& \mathrm{B} 0=\mathrm{G} 00+\mathrm{u} 0 \\
& \mathrm{~B} 1=\mathrm{G} 10 \\
& \mathrm{~B} 2=\mathrm{G} 20+\mathrm{u} 2 \\
& \mathrm{~B} 3=\mathrm{G} 30 \\
& \mathrm{~B} 4=\mathrm{G} 40 \\
& \mathrm{~B} 5=\mathrm{G} 50
\end{aligned}
$$

LG_SAA has been centered around the group mean.

$$
\begin{aligned}
& \text { Mixed Model } \\
& \text { LG_CORT = G00 } \\
& \text { + G10*TIME } \\
& \text { + G20*QUAD_TIM } \\
& \text { + G30*LG_SAA } \\
& \text { + G40*SAAXTIME } \\
& \text { + G50*SAAXQ_TI } \\
& \text { + u0 + u2*QUAD_TIM + r }
\end{aligned}
$$

$\operatorname{sigma}^{\wedge} 2=0.01932$

Standard error of $\operatorname{sigma}^{\wedge} 2=0.00244$

$$
\begin{array}{lll}
\text { tau } & & \\
\text { INTRCPT1,B0 } & 0.03054 & -0.00250 \\
\text { QUAD_TIM,B2 } & -0.00250 & 0.00081
\end{array}
$$

Standard errors of tau

$$
\begin{array}{lcc}
\text { INTRCPT1,B0 } & 0.00761 & 0.00121 \\
\text { QUAD_TIM,B2 } & 0.00121 & 0.00038
\end{array}
$$

tau (as correlations)

INTRCPT1,B0 $1.000-0.502$ 
QUAD_TIM,B2 -0.502 1.000

Random level-1 coefficient Reliability estimate

\begin{tabular}{lc}
\hline INTRCPT1, G0 & 0.874 \\
QUAD_TIM, G2 & 0.485 \\
\hline
\end{tabular}

The value of the log-likelihood function at iteration $13=5.810132 \mathrm{E}+001$

The outcome variable is LG_CORT

Final estimation of fixed effects:

\begin{tabular}{|c|c|c|c|c|c|}
\hline Fixed Effect & $\begin{array}{c}\text { Standard } \\
\text { Coefficient Erro }\end{array}$ & $\begin{array}{r}\text { Approx. } \\
\text { or } \quad \text { T-ratio }\end{array}$ & d.f. & P-value & \\
\hline \multicolumn{6}{|c|}{ For INTRCPT1, B0 } \\
\hline INTRCPT2, G00 & 0.448835 & 0.029635 & 15.145 & 41 & 0.000 \\
\hline \multicolumn{6}{|c|}{ For TIME slope, B1 } \\
\hline INTRCPT2, G10 & -0.004024 & 0.008943 & -0.450 & 121 & 0.654 \\
\hline \multicolumn{6}{|c|}{ For QUAD_TIM slope, B2 } \\
\hline INTRCPT2, G20 & 0.012700 & 0.007379 & 1.721 & 41 & 0.093 \\
\hline \multicolumn{6}{|c|}{ For LG_SAA slope, B3 } \\
\hline INTRCPT2, G30 & -0.044759 & 0.122530 & -0.365 & 121 & 0.716 \\
\hline \multicolumn{6}{|c|}{ For SAAXTIME slope, B4 } \\
\hline INTRCPT2, G40 & -0.062670 & 0.063017 & -0.994 & 121 & 0.322 \\
\hline \multicolumn{6}{|c|}{ For SAAXQ_TI slope, B5 } \\
\hline INTRCPT2, G50 & -0.022977 & 0.049630 & -0.463 & 121 & 0.644 \\
\hline
\end{tabular}

Final estimation of variance components:

Random Effect Standard Variance df Chi-square P-value Deviation Component

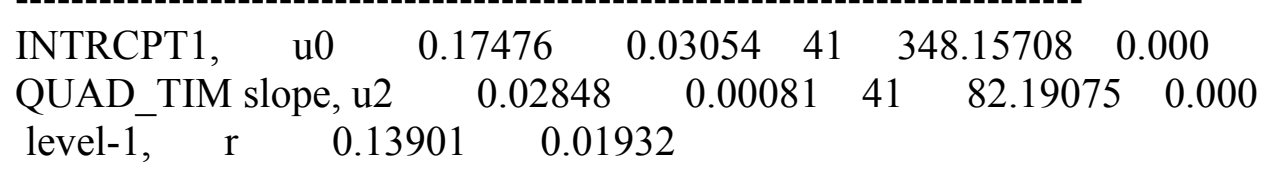

Statistics for the current model

Deviance $\quad=-116.202640$


Number of estimated parameters $=10$

Model comparison test

Chi-square statistic $=14.36050$

Degrees of freedom $=2$

P-value $\quad=0.001$

\section{Model 9}

Level-1 Model

LG_CORT $=\mathrm{B} 0+\mathrm{B} 1 *(\mathrm{TIME})+\mathrm{B} 2 *(\mathrm{QUAD}$ TIM $)+\mathrm{B} 3 *(\mathrm{LG}$ SAA $)+\mathrm{B} 4 *(\mathrm{SAAXTIME})+$ $\mathrm{B} 5 *(\mathrm{SAAX} \overline{\mathrm{X}} \mathrm{TI})+\mathrm{r}$

Level-2 Model

$$
\begin{aligned}
& \mathrm{B} 0=\mathrm{G} 00+\mathrm{G} 01 *(\mathrm{INTERNAL})+\mathrm{u} 0 \\
& \mathrm{~B} 1=\mathrm{G} 10 \\
& \mathrm{~B} 2=\mathrm{G} 20+\mathrm{u} 2 \\
& \mathrm{~B} 3=\mathrm{G} 30 \\
& \mathrm{~B} 4=\mathrm{G} 40 \\
& \mathrm{~B} 5=\mathrm{G} 50
\end{aligned}
$$

LG_SAA has been centered around the group mean.

INTERNAL has been centered around the grand mean.

$$
\begin{aligned}
& \text { Mixed Model } \\
& \text { LG_CORT }=\text { G00 + G01*INTERNAL } \\
& \text { + G10*TIME } \\
& \text { + G20*QUAD_TIM } \\
& \text { + G30*LG_SAA } \\
& \text { + G40*SAAXTIME } \\
& \text { + G50*SAAXQ_TI } \\
& \text { + u0 + u2*QUAD_TIM + r }
\end{aligned}
$$

$\operatorname{sigma}^{\wedge} 2=0.01932$

Standard error of sigma^ $2=0.00244$

tau

$$
\begin{array}{lll}
\text { INTRCPT1,B0 } & 0.03055 & -0.00250 \\
\text { QUAD_TIM,B2 } & -0.00250 & 0.00081
\end{array}
$$

Standard errors of tau 
$\begin{array}{lll}\text { INTRCPT1,B0 } & 0.00761 \quad 0.00121\end{array}$

QUAD_TIM,B2 $0.00121 \quad 0.00038$

tau (as correlations)

INTRCPT1,B0 $1.000-0.503$

QUAD_TIM,B2 -0.503 1.000

Random level-1 coefficient Reliability estimate

$\begin{array}{lc}\text { INTRCPT1, G0 } & 0.874 \\ \text { QUAD_TIM, G2 } & 0.485\end{array}$

The value of the log-likelihood function at iteration $13=5.810440 \mathrm{E}+001$

The outcome variable is LG_CORT

Final estimation of fixed effects:

\begin{tabular}{|c|c|c|c|c|c|}
\hline Fixed Effect & $\begin{array}{c}\text { Standard } \\
\text { Coefficient Erro }\end{array}$ & $\begin{array}{r}\text { Approx. } \\
\text { or } \quad \text { T-ratio }\end{array}$ & d.f. & P-value & \\
\hline \multicolumn{6}{|c|}{ For INTRCPT1, B0 } \\
\hline INTRCPT2, G00 & 0.448960 & 0.029681 & 15.126 & 40 & 0.000 \\
\hline INTERNAL, G01 & -0.000395 & 0.005031 & -0.079 & 40 & 0.938 \\
\hline \multicolumn{6}{|c|}{ For TIME slope, B1 } \\
\hline INTRCPT2, G10 & -0.004022 & 0.008943 & -0.450 & 121 & 0.654 \\
\hline \multicolumn{6}{|c|}{ For QUAD_TIM slope, B2 } \\
\hline INTRCPT̄2, G20 & 0.012689 & 0.007379 & 1.720 & 41 & 0.093 \\
\hline \multicolumn{6}{|c|}{ For LG_SAA slope, B3 } \\
\hline INTRCPT2, G30 & -0.044327 & 0.122640 & -0.361 & 121 & 0.718 \\
\hline \multicolumn{6}{|c|}{ For SAAXTIME slope, B4 } \\
\hline INTRCPT2, G40 & -0.062228 & 0.063271 & -0.984 & 121 & 0.327 \\
\hline \multicolumn{6}{|c|}{ For SAAXQ TI slope, B5 } \\
\hline INTRCPT2, G50 & -0.022746 & 0.049745 & -0.457 & 121 & 0.648 \\
\hline
\end{tabular}

Final estimation of variance components:

Random Effect Standard Variance df Chi-square P-value Deviation Component

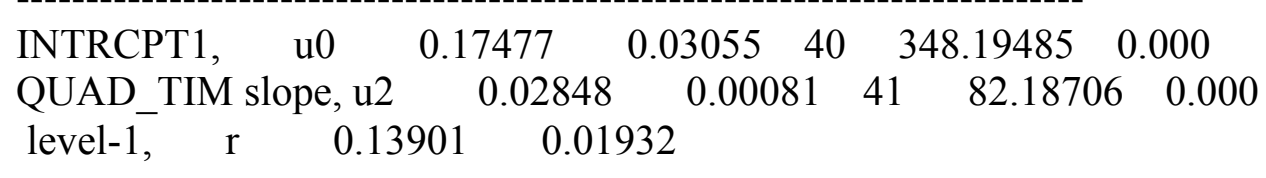


Statistics for the current model

Deviance $=-116.208801$

Number of estimated parameters $=11$

Model comparison test

Chi-square statistic $=0.18218$

Degrees of freedom $=0$

P-value $\quad=\quad>.500$

\section{Model 10}

Level-1 Model

LG_CORT $=$ B0 + B1*(TIME $)+$ B2* $\left(\mathrm{QUAD} \_\mathrm{TIM}\right)+\mathrm{B} 3 *($ LG_SAA $)+\mathrm{B} 4 *(\mathrm{SAAXTIME})+$ $\mathrm{B} 5 *(\mathrm{SAAXQ} \mathrm{TI})+\mathrm{r}$

Level-2 Model

$$
\begin{aligned}
& \mathrm{B} 0=\mathrm{G} 00+\mathrm{G} 01 *(\mathrm{INTERNAL})+\mathrm{u} 0 \\
& \mathrm{~B} 1=\mathrm{G} 10+\mathrm{G} 11 *(\mathrm{INTERNAL}) \\
& \mathrm{B} 2=\mathrm{G} 20+\mathrm{G} 21 *(\mathrm{INTERNAL})+\mathrm{u} 2 \\
& \mathrm{~B} 3=\mathrm{G} 30 \\
& \mathrm{~B} 4=\mathrm{G} 40 \\
& \mathrm{~B} 5=\mathrm{G} 50
\end{aligned}
$$

LG_SAA has been centered around the group mean.

INTERNAL has been centered around the grand mean.

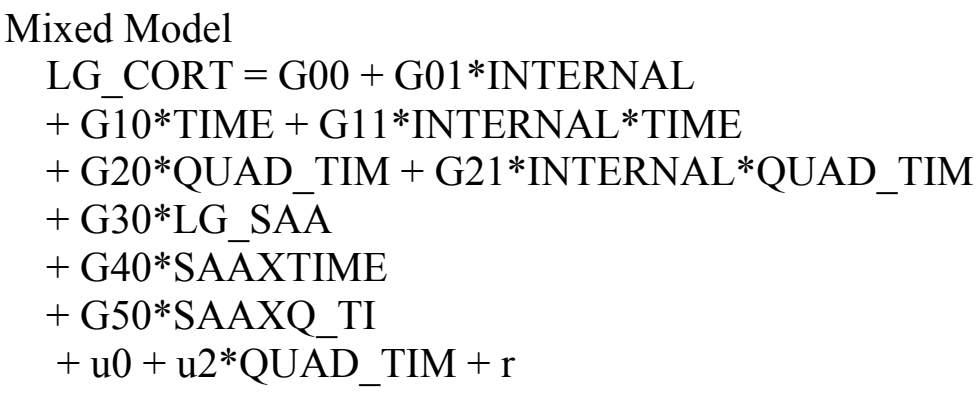

$\operatorname{sigma}^{\wedge} 2=0.01906$

Standard error of $\operatorname{sigma}^{\wedge} 2=0.00240$

tau 


$$
\begin{array}{llc}
\text { INTRCPT1,B0 } & 0.03079 & -0.00250 \\
\text { QUAD_TIM,B2 } & -0.00250 & 0.00082
\end{array}
$$

Standard errors of tau

$\begin{array}{lcc}\text { INTRCPT1,B0 } & 0.00765 & 0.00122 \\ \text { QUAD_TIM,B2 } & 0.00122 & 0.00038\end{array}$

tau (as correlations)

INTRCPT1,B0 $1.000-0.496$

QUAD_TIM,B2 -0.496 1.000

Random level-1 coefficient Reliability estimate

\begin{tabular}{lc} 
INTRCPT1, G0 & 0.876 \\
QUAD_TIM, G2 & 0.492 \\
\hline
\end{tabular}

The value of the log-likelihood function at iteration $13=5.885410 \mathrm{E}+001$

The outcome variable is LG_CORT

\begin{tabular}{|c|c|c|c|c|c|}
\hline Fixed Effect & $\begin{array}{c}\text { Standard } \\
\text { Coefficient Erro }\end{array}$ & $\begin{array}{r}\text { Approx. } \\
\text { or } \quad \text { T-ratio }\end{array}$ & d.f. & P-value & \\
\hline \multicolumn{6}{|c|}{ For INTRCPT1, B0 } \\
\hline INTRCPT2, G00 & 0.448384 & 0.029752 & 15.071 & 40 & 0.000 \\
\hline INTERNAL, G01 & 0.001245 & 0.005287 & 0.235 & 40 & 0.815 \\
\hline \multicolumn{6}{|c|}{ For TIME slope, B1 } \\
\hline INTRCPT2, G10 & -0.003575 & 0.008889 & -0.402 & 120 & 0.688 \\
\hline INTERNAL, G11 & -0.002026 & 0.001686 & -1.201 & 120 & 0.232 \\
\hline \multicolumn{6}{|c|}{ For QUAD TIM slope, B2 } \\
\hline INTRCPT̄2, G20 & 0.013038 & 0.007380 & 1.767 & 40 & 0.085 \\
\hline INTERNAL, G21 & -0.001124 & 0.001354 & -0.830 & 40 & 0.411 \\
\hline \multicolumn{6}{|c|}{ For LG_SAA slope, B3 } \\
\hline INTRCPT2, G30 & -0.032218 & 0.122697 & -0.263 & 120 & 0.793 \\
\hline \multicolumn{6}{|c|}{ For SAAXTIME slope, B4 } \\
\hline INTRCPT2, G40 & -0.033735 & 0.067154 & -0.502 & 120 & 0.616 \\
\hline \multicolumn{6}{|c|}{ For SAAXQ TI slope, B5 } \\
\hline INTRCPT2, G50 & -0.009592 & 0.050792 & -0.189 & 120 & 0.851 \\
\hline
\end{tabular}

Final estimation of fixed effects:

Final estimation of variance components:

Random Effect Standard Variance df Chi-square P-value


Deviation Component

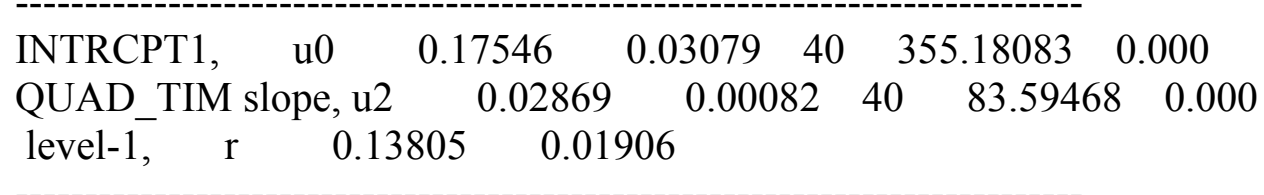

Statistics for the current model

Deviance $\quad=-117.708203$

Number of estimated parameters $=13$

Model comparison test

Chi-square statistic $=1.49940$

Degrees of freedom $=2$

P-value $\quad=\quad>.500$

\section{Model 11}

Level-1 Model

LG_CORT $=$ B0 + B1*(TIME $)+$ B2* $($ QUAD_TIM $)+$ B3* $($ LG_SAA $)+$ B4 $*($ SAAXTIME $)+$ $\mathrm{B} 5 *(\mathrm{SAAX} \overline{\mathrm{X}} \mathrm{TI})+\mathrm{r}$

Level-2 Model

$$
\begin{aligned}
& \mathrm{B} 0=\mathrm{G} 00+\mathrm{G} 01 *(\text { INTERNAL })+\mathrm{u} 0 \\
& \mathrm{~B} 1=\mathrm{G} 10+\mathrm{G} 11 *(\text { INTERNAL }) \\
& \mathrm{B} 2=\mathrm{G} 20+\mathrm{G} 21 *(\text { INTERNAL })+\mathrm{u} 2 \\
& \mathrm{~B} 3=\mathrm{G} 30+\mathrm{G} 31 *(\text { INTERNAL }) \\
& \mathrm{B} 4=\mathrm{G} 40 \\
& \mathrm{~B} 5=\mathrm{G} 50
\end{aligned}
$$

LG_SAA has been centered around the group mean.

INTERNAL has been centered around the grand mean.

$$
\begin{aligned}
& \text { Mixed Model } \\
& \text { LG_CORT }=\text { G00 + G01*INTERNAL } \\
& \text { + G10*TIME + G1 } 1 * \text { INTERNAL*TIME } \\
& \text { + G20*QUAD_TIM + G21*INTERNAL*QUAD_TIM } \\
& \text { + G30*LG_SAA + G31*INTERNAL*LG_SAA } \\
& \text { + G40*SAAXTIME } \\
& \text { + G50*SAAXQ_TI } \\
& \text { + u0 + u2*QUAD_TIM + r }
\end{aligned}
$$


$\operatorname{sigma}^{\wedge} 2=0.01867$

Standard error of sigma^ $2=0.00236$

tau

INTRCPT1,B0 $\quad 0.03084 \quad-0.00277$

QUAD_TIM,B2 $\quad-0.00277 \quad 0.00082$

Standard errors of tau

$\begin{array}{lll}\text { INTRCPT1,B0 } & 0.00764 & 0.00122\end{array}$

QUAD_TIM,B2 $0.00122 \quad 0.00037$

tau (as correlations)

INTRCPT1,B0 $1.000-0.551$

QUAD_TIM,B2 -0.551 1.000

Random level-1 coefficient Reliability estimate

$\begin{array}{lc}\text { INTRCPT1, G0 } & 0.879 \\ \text { QUAD_TIM, G2 } & 0.496\end{array}$

The value of the log-likelihood function at iteration $13=6.093949 \mathrm{E}+001$

The outcome variable is LG_CORT

Final estimation of fixed effects:

\begin{tabular}{|c|c|c|c|c|c|}
\hline Fixed Effect & $\begin{array}{c}\text { Standard } \\
\text { Coefficient Erro }\end{array}$ & $\begin{array}{r}\text { Approx. } \\
\text { or } \quad \text { T-ratio }\end{array}$ & d.f. & P-value & \\
\hline \multicolumn{6}{|c|}{ For INTRCPT1, B0 } \\
\hline INTRCPT2, G00 & 0.445692 & 0.029745 & 14.984 & 40 & 0.000 \\
\hline INTERNAL, G01 & 0.000538 & 0.005291 & 0.102 & 40 & 0.919 \\
\hline \multicolumn{6}{|c|}{ For TIME slope, B1 } \\
\hline INTRCPT2, G10 & -0.000836 & 0.008895 & -0.094 & 119 & 0.925 \\
\hline INTERNAL, G11 & -0.001669 & 0.001678 & -0.995 & 119 & 0.322 \\
\hline \multicolumn{6}{|c|}{ For QUAD TIM slope, B2 } \\
\hline INTRCPT̄2, G20 & 0.014052 & 0.007342 & 1.914 & 40 & 0.063 \\
\hline INTERNAL, G21 & -0.001257 & 0.001344 & -0.935 & 40 & 0.355 \\
\hline \multicolumn{6}{|c|}{ For LG_SAA slope, B3 } \\
\hline INTRCPT2, G30 & -0.016914 & 0.121118 & -0.140 & 119 & 0.889 \\
\hline INTERNAL, G31 & 0.043570 & 0.021039 & 2.071 & 119 & 0.041 \\
\hline \multicolumn{6}{|c|}{ For SAAXTIME slope, B4 } \\
\hline INTRCPT2, G40 & -0.012456 & 0.067214 & -0.185 & 119 & 0.853 \\
\hline
\end{tabular}


For SAAXQ_TI slope, B5

$\begin{array}{llllll}\text { INTRCPT2, G50 } & -0.010187 & 0.049992 & -0.204 & 119 & 0.839\end{array}$

Final estimation of variance components:

Random Effect Standard Variance df Chi-square P-value

Deviation Component

$\begin{array}{lllllll}\text { INTRCPT1, u0 } & 0.17562 & 0.03084 & 40 & 361.98112 & 0.000\end{array}$

$\begin{array}{llllll}\text { QUAD_TIM slope, u2 } & 0.02862 & 0.00082 & 40 & 83.94589 & 0.000\end{array}$

$\begin{array}{llll}\text { level-1, } & \text { r } & 0.13662 & 0.01867\end{array}$

Statistics for the current model

Deviance $\quad=-121.878977$

Number of estimated parameters $=14$

Model comparison test

Chi-square statistic $=4.50480$
Degrees of freedom $=1$
P-value $=0.032$

\section{Model 12}

Level-1 Model

LG_CORT $=$ B0 + B $1 *(T I M E)+B 2 *\left(Q U A D \_T I M\right)+B 3 *($ LG_SAA $)+$ B4 $*($ SAAXTIME $)+$ $\mathrm{B} 5 *\left(\mathrm{SAAX} \mathrm{Q}_{-} \mathrm{TI}\right)+\mathrm{r}$

Level-2 Model

$$
\begin{aligned}
& \mathrm{B} 0=\mathrm{G} 00+\mathrm{G} 01 *(\text { INTERNAL })+\mathrm{u} 0 \\
& \mathrm{~B} 1=\mathrm{G} 10+\mathrm{G} 11 *(\text { INTERNAL }) \\
& \mathrm{B} 2=\mathrm{G} 20+\mathrm{G} 21 *(\text { INTERNAL })+\mathrm{u} 2 \\
& \mathrm{~B} 3=\mathrm{G} 30+\mathrm{G} 31 *(\text { INTERNAL }) \\
& \mathrm{B} 4=\mathrm{G} 40+\mathrm{G} 41 *(\text { INTERNAL }) \\
& \mathrm{B} 5=\mathrm{G} 50+\mathrm{G} 51 *(\text { INTERNAL })
\end{aligned}
$$

LG_SAA has been centered around the group mean.

INTERNAL has been centered around the grand mean.

Mixed Model 


$$
\begin{aligned}
& \text { LG_CORT }=\text { G00 + G01*INTERNAL } \\
& \text { + G10*TIME + G11*INTERNAL*TIME } \\
& \text { + G20*QUAD_TIM + G21*INTERNAL*QUAD_TIM } \\
& \text { + G30*LG_SAA + G31*INTERNAL*LG_SAA } \\
& \text { + G40*SAAXTIME + G41*INTERNAL*SAAXTIME } \\
& \text { + G50*SAAXQ_TI + G51*INTERNAL*SAAXQ_TI } \\
& \text { + u0 + u2*QUAD_TIM + r }
\end{aligned}
$$

$\operatorname{sigma}^{\wedge} 2=0.01889$

Standard error of sigma^ $2=0.00238$

$$
\begin{array}{llc}
\text { tau } & & \\
\text { INTRCPT1,B0 } & 0.02959 & -0.00266 \\
\text { QUAD_TIM,B2 } & -0.00266 & 0.00074
\end{array}
$$

Standard errors of tau

$$
\begin{array}{lcc}
\text { INTRCPT1,B0 } & 0.00738 & 0.00117 \\
\text { QUAD_TIM,B2 } & 0.00117 & 0.00036
\end{array}
$$

tau (as correlations)

INTRCPT1,B0 $1.000-0.570$

QUAD_TIM,B2 -0.570 1.000

Random level-1 coefficient Reliability estimate

$\begin{array}{lc}\text { INTRCPT1, G0 } & 0.873 \\ \text { QUAD_TIM, G2 } & 0.467\end{array}$

The value of the log-likelihood function at iteration $13=6.180150 \mathrm{E}+001$

The outcome variable is LG_CORT

Final estimation of fixed effects:

\begin{tabular}{lllllll} 
& \multicolumn{2}{c}{ Standard } & Approx. & & \\
Fixed Effect & Coefficient & Error & T-ratio & d.f. & P-value & \\
\hdashline For INTRCPT1, B0 & & & & & \\
INTRCPT2, G00 & 0.444012 & 0.029287 & 15.161 & 40 & 0.000 \\
INTERNAL, G01 & -0.000218 & 0.005241 & -0.042 & 40 & 0.967 \\
For TIME slope, B1 & & & & & \\
INTRCPT2, G10 & -0.004400 & 0.009912 & -0.444 & 117 & 0.658 \\
INTERNAL, G11 & -0.002409 & 0.001927 & -1.250 & 117 & 0.214 \\
For QUAD_TIM slope, B2 & & & & &
\end{tabular}


$\begin{array}{llllll}\text { INTRCPT2, G20 } & 0.013204 & 0.007497 & 1.761 & 40 & 0.086\end{array}$

$\begin{array}{llllll}\text { INTERNAL, G21 } & -0.001491 & 0.001391 & -1.072 & 40 & 0.290\end{array}$

For_LG_SAA slope, B3

$\begin{array}{llllll}\text { INTRCPT2, G30 } & 0.027382 & 0.125827 & 0.218 & 117 & 0.828\end{array}$

$\begin{array}{llllll}\text { INTERNAL, G31 } & 0.065522 & 0.027412 & 2.390 & 117 & 0.018\end{array}$

For SAAXTIME slope, B4

$\begin{array}{llllll}\text { INTRCPT2, G40 } & 0.005236 & 0.070279 & 0.075 & 117 & 0.941\end{array}$

$\begin{array}{llllll}\text { INTERNAL, G41 } & 0.014429 & 0.016416 & 0.879 & 117 & 0.381\end{array}$

For SAAXQ_TI slope, B5

$\begin{array}{llllll}\text { INTRCPT2, G50 } & -0.015665 & 0.051783 & -0.303 & 117 & 0.763\end{array}$

$\begin{array}{llllll}\text { INTERNAL, G51 } & -0.002451 & 0.010865 & -0.226 & 117 & 0.822\end{array}$

Final estimation of variance components:

Random Effect Standard Variance df Chi-square P-value Deviation Component

$\begin{array}{lllllll}\text { INTRCPT1, u0 } & 0.17201 & 0.02959 & 40 & 345.46384 & 0.000\end{array}$

QUAD_TIM slope, u2 $\quad 0.02716 \quad 0.00074 \quad 40 \quad 79.49760 \quad 0.000$

$\begin{array}{llll}\text { level-1, } & r & 0.13745 & 0.01889\end{array}$

Statistics for the current model

Deviance $\quad=-123.602999$

Number of estimated parameters $=16$

Model comparison test

Chi-square statistic $=1.72402$

Degrees of freedom $=2$

P-value $\quad=\quad>.500$

\section{Model 13}

Level-1 Model

$$
\text { LG_CORT }=\text { B0 + B1 } *(\text { TIME })+\text { B } 2 *\left(Q U A D \_T I M\right)+B 3 *\left(L G \_S A A\right)+r
$$

Level-2 Model

$$
\begin{aligned}
& \mathrm{B} 0=\mathrm{G} 00+\mathrm{u} 0 \\
& \mathrm{~B} 1=\mathrm{G} 10 \\
& \mathrm{~B} 2=\mathrm{G} 20+\mathrm{u} 2 \\
& \mathrm{~B} 3=\mathrm{G} 30
\end{aligned}
$$


LG_SAA has been centered around the group mean.

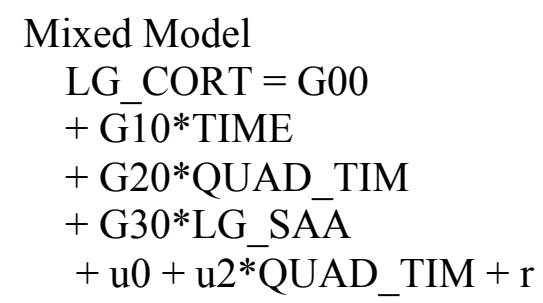

$\operatorname{sigma}^{\wedge} 2=0.01942$

Standard error of $\operatorname{sigma}^{\wedge} 2=\quad 0.00245$

tau

INTRCPT1,B0 $0.03106 \quad-0.00251$

QUAD_TIM,B2 $-0.00251 \quad 0.00080$

Standard errors of tau

INTRCPT1,B0 $0.00773 \quad 0.00122$

QUAD_TIM,B2 $0.00122 \quad 0.00038$

tau (as correlations)

INTRCPT1,B0 $1.000-0.504$

QUAD_TIM,B2 -0.504 1.000

Random level-1 coefficient Reliability estimate

$\begin{array}{lc}\text { INTRCPT1, G0 } & 0.875 \\ \text { QUAD_TIM, G2 } & 0.479\end{array}$

The value of the log-likelihood function at iteration $8=5.760570 \mathrm{E}+001$

The outcome variable is LG_CORT

Final estimation of fixed effects:

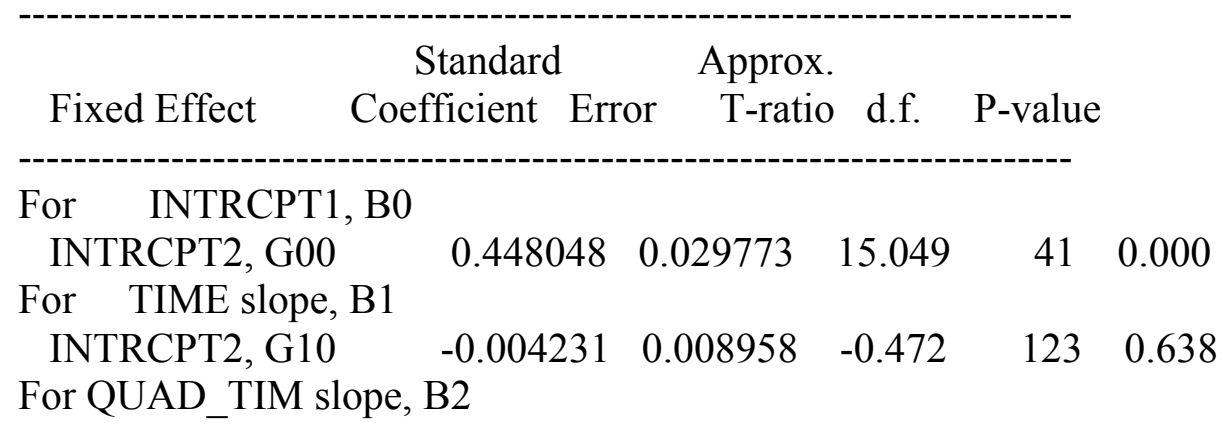


$\begin{array}{llllll}\text { INTRCPT2, G20 } & 0.012288 & 0.007306 & 1.682 & 41 & 0.100\end{array}$

For LG_SAA slope, B3

$\begin{array}{llllll}\text { INTRCPT2, G30 } & 0.023959 & 0.100829 & 0.238 & 123 & 0.813\end{array}$

Final estimation of variance components:

Random Effect Standard Variance df Chi-square P-value

Deviation Component

$\begin{array}{llllll}\text { INTRCPT1, u0 } & 0.17623 & 0.03106 & 41 & 351.79917 & 0.000\end{array}$

QUAD_TIM slope, u2 $\quad 0.02822 \quad 0.00080 \quad 41 \quad 81.23429 \quad 0.000$

$\begin{array}{llll}\text { level-1, } & \mathrm{r} & 0.13935 & 0.01942\end{array}$

Statistics for the current model

Deviance $\quad=-115.211409$

Number of estimated parameters $=8$

Model comparison test

Chi-square statistic $=14.09680$

Degrees of freedom $=2$

$\mathrm{P}$-value $\quad=0.001$

\section{Model 14}

Level-1 Model

$$
\text { LG_CORT }=\text { B } 0+\text { B } 1 *(\text { TIME })+\text { B2 } *\left(Q U A D \_T I M\right)+B 3 *\left(L G \_S A A\right)+r
$$

Level-2 Model

$$
\begin{aligned}
& \mathrm{B} 0=\mathrm{G} 00+\mathrm{G} 01 *(\text { INTERNAL })+\mathrm{u} 0 \\
& \mathrm{~B} 1=\mathrm{G} 10 \\
& \mathrm{~B} 2=\mathrm{G} 20+\mathrm{u} 2 \\
& \mathrm{~B} 3=\mathrm{G} 30
\end{aligned}
$$

LG_SAA has been centered around the group mean.

INTERNAL has been centered around the grand mean.

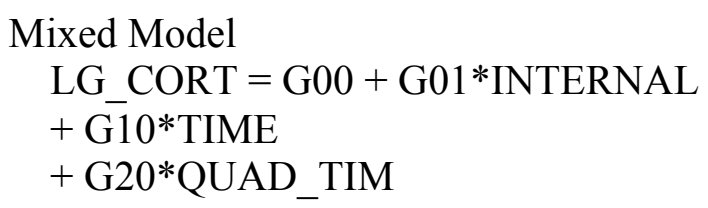




$$
\begin{aligned}
& +\mathrm{G} 30 * \text { LG_SAA } \\
& +\mathrm{u} 0+\mathrm{u} 2 * \mathrm{QUAD} \text { TIM }+\mathrm{r}
\end{aligned}
$$

$\operatorname{sigma}^{\wedge} 2=0.01942$

Standard error of $\operatorname{sigma}^{\wedge} 2=\quad 0.00245$

tau

INTRCPT1,B0 $0.03105 \quad-0.00252$

QUAD_TIM,B2 -0.00252 0.00080

Standard errors of tau

$\begin{array}{lcc}\text { INTRCPT1,B0 } & 0.00773 & 0.00122 \\ \text { QUAD_TIM,B2 } & 0.00122 & 0.00038\end{array}$

tau (as correlations)

INTRCPT1,B0 1.000 -0.506

QUAD_TIM,B2 -0.506 1.000

Random level-1 coefficient Reliability estimate

$\begin{array}{lc}\text { INTRCPT1, G0 } & 0.875 \\ \text { QUAD_TIM, G2 } & 0.479\end{array}$

The value of the log-likelihood function at iteration $8=5.761922 \mathrm{E}+001$

The outcome variable is LG_CORT

\begin{tabular}{|c|c|c|c|c|c|}
\hline Fixed Effect & $\begin{array}{c}\text { Standard } \\
\text { Coefficient Erro }\end{array}$ & $\begin{array}{r}\text { Approx. } \\
\text { or } \quad \text { T-ratio }\end{array}$ & d.f. & P-value & \\
\hline \multicolumn{6}{|c|}{ For INTRCPT1, B0 } \\
\hline INTRCPT2, G00 & 0.448315 & 0.029814 & 15.037 & 40 & 0.000 \\
\hline INTERNAL, G01 & -0.000831 & 0.005046 & -0.165 & 40 & 0.870 \\
\hline \multicolumn{6}{|c|}{ For TIME slope, B1 } \\
\hline INTRCPT2, G10 & -0.004224 & 0.008959 & -0.471 & 123 & 0.638 \\
\hline \multicolumn{6}{|c|}{ For QUAD_TIM slope, B2 } \\
\hline INTRCPT2, G20 & 0.012269 & 0.007306 & 1.679 & 41 & 0.101 \\
\hline \multicolumn{6}{|c|}{ For LG_SAA slope, B3 } \\
\hline INTRCPT2, G30 & 0.023906 & 0.100823 & 0.237 & 123 & 0.813 \\
\hline
\end{tabular}

Final estimation of fixed effects:

Final estimation of variance components: 
Random Effect Standard Variance df Chi-square P-value Deviation Component

$\begin{array}{llllllll}\text { INTRCPT1, u0 } & 0.17620 & 0.03105 & 40 & 351.67262 & 0.000 \\ \text { QUAD_TIM slope, u2 } & 0.02821 & 0.00080 & 41 & 81.22331 & 0.000 \\ \text { level-1, } \quad \mathrm{r} & 0.13936 & 0.01942 & & & & \end{array}$

Statistics for the current model

Deviance $\quad=-115.238441$

Number of estimated parameters $=9$

Model comparison test

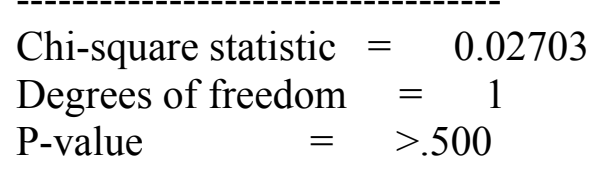

\section{Model 15}

Level-1 Model

$$
\text { LG_CORT }=\text { B0 + B1 } *(\text { TIME })+\text { B2 } *\left(Q U A D \_T I M\right)+B 3 *\left(L G \_S A A\right)+r
$$

Level-2 Model

$$
\begin{aligned}
& \mathrm{B} 0=\mathrm{G} 00+\mathrm{G} 01 *(\text { INTERNAL })+\mathrm{u} 0 \\
& \mathrm{~B} 1=\mathrm{G} 10+\mathrm{G} 11 *(\text { INTERNAL }) \\
& \mathrm{B} 2=\mathrm{G} 20+\mathrm{G} 21 *(\text { INTERNAL })+\mathrm{u} 2 \\
& \mathrm{~B} 3=\mathrm{G} 30
\end{aligned}
$$

LG_SAA has been centered around the group mean.

INTERNAL has been centered around the grand mean.

$$
\begin{aligned}
& \text { Mixed Model } \\
& \text { LG_CORT }=\mathrm{G} 00+\mathrm{G} 01 * \text { INTERNAL } \\
& + \text { G10*TIME + G11*INTERNAL*TIME } \\
& \quad+\mathrm{G} 20 * \text { QUAD_TIM }+ \text { G2 } 1 * \text { INTERNAL*QUAD_TIM } \\
& \quad+\mathrm{G} 30 * \text { LG_SAA } \\
& \quad+\mathrm{u} 0+\mathrm{u} 2 * \text { QUUAD_TIM }+\mathrm{r}
\end{aligned}
$$

$\operatorname{sigma}^{\wedge} 2=0.01908$ 
Standard error of sigma ${ }^{\wedge}=\quad 0.00241$

$\begin{array}{llc}\text { tau } & & \\ \text { INTRCPT1,B0 } & 0.03110 & -0.00252 \\ \text { QUAD_TIM,B2 } & -0.00252 & 0.00081\end{array}$

Standard errors of tau

$\begin{array}{lcc}\text { INTRCPT1,B0 } & 0.00772 & 0.00122 \\ \text { QUAD_TIM,B2 } & 0.00122 & 0.00038\end{array}$

tau (as correlations)

INTRCPT1,B0 $1.000-0.501$

QUAD_TIM,B2 -0.501 1.000

Random level-1 coefficient Reliability estimate

$\begin{array}{lc} & \\ \text { INTRCPT1, G0 } & 0.877 \\ \text { QUAD_TIM, G2 } & 0.489\end{array}$

The value of the log-likelihood function at iteration $8=5.872260 \mathrm{E}+001$

The outcome variable is LG_CORT

Final estimation of fixed effects:

\begin{tabular}{|c|c|c|c|c|c|c|}
\hline Fixed Effect & & $\begin{array}{l}\text { Standard } \\
\text { efficient Erro }\end{array}$ & $\begin{array}{r}\text { Approx. } \\
\text { or } \quad \text { T-ratio }\end{array}$ & d.f. & P-value & \\
\hline \multicolumn{7}{|c|}{ For INTRCPT1, B0 } \\
\hline \multicolumn{2}{|c|}{ INTRCPT2, G00 } & 0.447878 & 0.029804 & 15.027 & 40 & 0.000 \\
\hline \multirow{2}{*}{\multicolumn{2}{|c|}{$\begin{array}{l}\text { INTERNAL, G01 } \\
\text { For TIME slone B1 }\end{array}$}} & 0.001223 & 0.005309 & 0.230 & 40 & 0.819 \\
\hline \multirow{2}{*}{\multicolumn{2}{|c|}{ INTRCPT2, G10 }} & & & & & \\
\hline & & -0.003589 & 0.008890 & -0.404 & 122 & 0.687 \\
\hline INTERNAL, $\mathrm{C}$ & & -0.002327 & 0.001579 & -1.474 & 122 & 0.143 \\
\hline \multicolumn{7}{|c|}{ For QUAD_TIM slope, B2 } \\
\hline \multicolumn{2}{|c|}{ INTRCPT̄2, G20 } & 0.012807 & 0.007307 & 1.753 & 40 & 0.087 \\
\hline \multirow{2}{*}{\multicolumn{2}{|c|}{$\begin{array}{l}\text { INTERNAL, G21 } \\
\text { For LG_SAA slope, B }\end{array}$}} & -0.001221 & 0.001321 & -0.924 & 40 & 0.361 \\
\hline & & & & & & \\
\hline \multicolumn{2}{|c|}{ INTRCPT2, G30 } & 0.003687 & 0.101013 & 0.037 & 122 & 0.971 \\
\hline
\end{tabular}

Final estimation of variance components:

Random Effect Standard Variance df Chi-square P-value Deviation Component 
$\begin{array}{lllllll}\text { INTRCPT1, u0 } & 0.17634 & 0.03110 & 40 & 358.05121 & 0.000\end{array}$ QUAD_TIM slope, u2 $\quad 0.02854 \quad 0.00081 \quad 40 \quad 83.17319 \quad 0.000$ $\begin{array}{llll}\text { level-1, } & \mathrm{r} & 0.13812 & 0.01908\end{array}$

Statistics for the current model

Deviance $\quad=-117.445210$

Number of estimated parameters $=11$

Model comparison test

Chi-square statistic $=2.20677$

Degrees of freedom $=2$

P-value $\quad=0.332$

\section{Model 16}

Level-1 Model

$$
\text { LG_CORT }=\text { B0 }+ \text { B } 1 *(\text { TIME })+\text { B2 } *\left(Q U A D \_T I M\right)+B 3 *\left(L G \_S A A\right)+r
$$

Level-2 Model

$$
\begin{aligned}
& \mathrm{B} 0=\mathrm{G} 00+\mathrm{G} 01 *(\text { INTERNAL })+\mathrm{u} 0 \\
& \mathrm{~B} 1=\mathrm{G} 10 \\
& \mathrm{~B} 2=\mathrm{G} 20+\mathrm{u} 2 \\
& \mathrm{~B} 3=\mathrm{G} 30+\mathrm{G} 31 *(\text { INTERNAL })
\end{aligned}
$$

LG_SAA has been centered around the group mean.

INTERNAL has been centered around the grand mean.

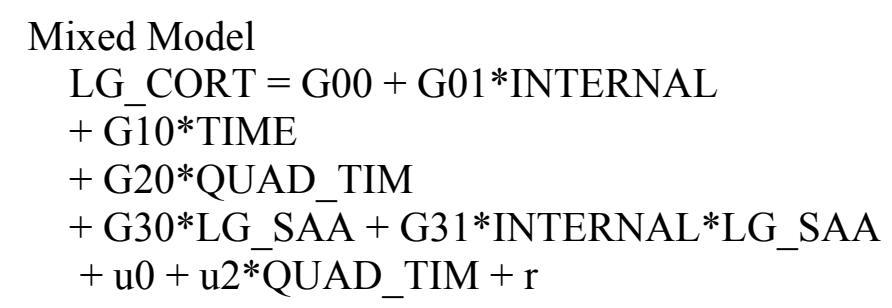

$\operatorname{sigma}^{\wedge} 2=0.01883$

Standard error of sigma^ $2=0.00238$

tau 


$\begin{array}{llc}\text { INTRCPT1,B0 } & 0.03088 & -0.00276 \\ \text { QUAD_TIM,B2 } & -0.00276 & 0.00082\end{array}$

Standard errors of tau

$\begin{array}{lll}\text { INTRCPT1,B0 } & 0.00766 & 0.00122\end{array}$

$\begin{array}{lll}\text { QUAD_TIM,B2 } & 0.00122 & 0.00037\end{array}$

tau (as correlations)

INTRCPT1,B0 $1.000-0.548$

QUAD_TIM,B2 -0.548 1.000

Random level-1 coefficient Reliability estimate

\begin{tabular}{lc} 
INTRCPT1, G0 & 0.878 \\
QUAD_TIM, G2 & 0.495 \\
\hline$-ב-ב-$
\end{tabular}

The value of the log-likelihood function at iteration $8=6.014842 \mathrm{E}+001$

The outcome variable is LG_CORT

Final estimation of fixed effects:

\begin{tabular}{|c|c|c|c|c|c|}
\hline Fixed Effect & $\begin{array}{c}\text { Standard } \\
\text { Coefficient Erro }\end{array}$ & $\begin{array}{r}\text { Approx. } \\
\text { T-ratio }\end{array}$ & d.f. & P-value & \\
\hline \multicolumn{6}{|c|}{ For INTRCPT1, B0 } \\
\hline INTRCPT2, G0C & 0.446369 & 0.029684 & 15.037 & 40 & 0.000 \\
\hline INTERNAL, G0 & -0.001539 & 0.004988 & -0.309 & 40 & 0.759 \\
\hline \multicolumn{6}{|c|}{ For TIME slope, B1 } \\
\hline INTRCPT2, G10 & -0.001204 & 0.008923 & -0.135 & 122 & 0.893 \\
\hline \multicolumn{6}{|c|}{ For QUAD_TIM slope, B2 } \\
\hline INTRCPT2, G2C & 0.013705 & 0.007305 & 1.876 & 41 & 0.068 \\
\hline \multicolumn{6}{|c|}{ For LG_SAA slope, B3 } \\
\hline INTRCPT2, G3C & 0.007410 & 0.099490 & 0.074 & 122 & 0.941 \\
\hline INTERNAL, G3 & 0.046367 & 0.020353 & 2.278 & 122 & 0.024 \\
\hline
\end{tabular}

Final estimation of variance components:

Random Effect Standard Variance df Chi-square P-value
Deviation Component

\begin{tabular}{|c|c|c|c|c|}
\hline $\mathrm{u} 0$ & 0.17573 & $0.03088 \quad 40$ & 359.26699 & .000 \\
\hline IM slope, u2 & 0.02868 & $0.00082 \quad 41$ & 83.55906 & 0.000 \\
\hline
\end{tabular}


Statistics for the current model

Deviance $\quad=-120.296842$

Number of estimated parameters $=10$

Model comparison test

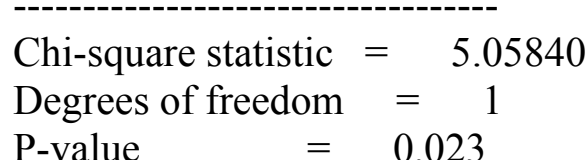

\section{Model 17}

Level-1 Model

$$
\text { LG_CORT }=\mathrm{B} 0+\mathrm{B} 1 *(\mathrm{TIME})+\mathrm{B} 2 *\left(\mathrm{QUAD} \_\mathrm{TIM}\right)+\mathrm{B} 3 *\left(\mathrm{LG} \_\mathrm{SAA}\right)+\mathrm{r}
$$

Level-2 Model

$$
\mathrm{B} 0=\mathrm{G} 00+\mathrm{G} 01 *(\mathrm{CHILD} \text { _EA })+\mathrm{G} 02 *\left(\mathrm{CHILD} \_\mathrm{WA}\right)+\mathrm{G} 03 *(\text { INTERNAL })+
$$
G04*(CHILD_SE)$$
+ \text { G05*(C)HILD_AG })+ \text { G06* }(\text { ETHNICIT })+\text { G07*(TIME_GAP })+u 0
$$$$
\mathrm{B} 1=\mathrm{G} 10
$$$$
\mathrm{B} 2=\mathrm{G} 20+\mathrm{u} 2
$$$$
\mathrm{B} 3=\mathrm{G} 30+\mathrm{G} 31 *(\text { INTERNAL })
$$

LG_SAA has been centered around the group mean.

INTERNAL has been centered around the grand mean.

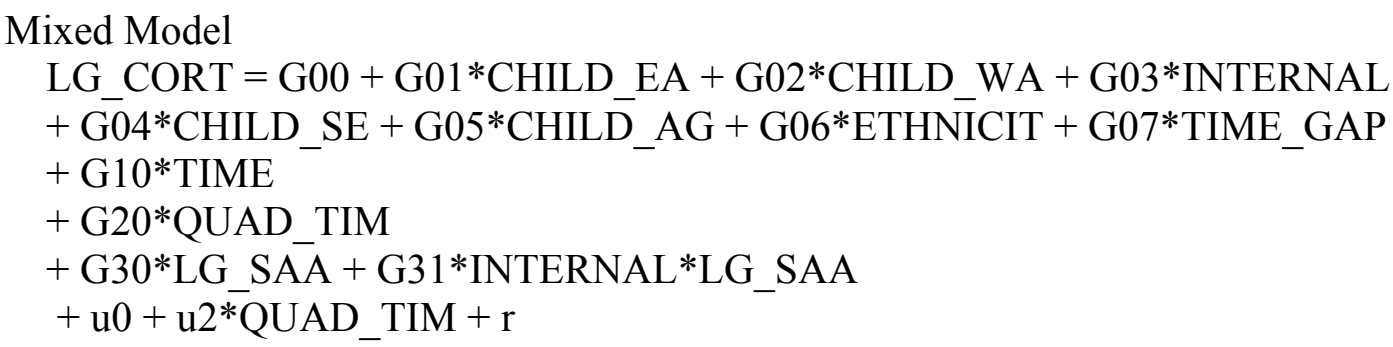

$\operatorname{sigma}^{\wedge} 2=0.01969$

Standard error of $\operatorname{sigma}^{\wedge} 2=0.00265$

tau

INTRCPT1,B0 $\quad 0.02477 \quad-0.00225$ 
QUAD_TIM,B2 $\quad-0.00225 \quad 0.00086$

Standard errors of tau

$\begin{array}{lll}\text { INTRCPT1,B0 } & 0.00679 & 0.00120\end{array}$

$\begin{array}{lll}\text { QUAD_TIM,B2 } & 0.00120 & 0.00042\end{array}$

tau (as correlations)

INTRCPT1,B0 $1.000-0.487$

QUAD_TIM,B2 -0.487 1.000

Random level-1 coefficient Reliability estimate

\begin{tabular}{lc} 
INTRCPT1, G0 & 0.846 \\
QUAD_TIM, G2 & 0.494 \\
\hline
\end{tabular}

Final estimation of fixed effects:

\begin{tabular}{|c|c|c|c|c|c|}
\hline Fixed Effect & $\begin{array}{c}\text { Standard } \\
\text { Coefficient Error }\end{array}$ & $\begin{array}{r}\text { Approx. } \\
\text { or } \quad \text { T-ratio }\end{array}$ & d.f. & P-value & \\
\hline \multicolumn{6}{|c|}{ For $\quad$ INTRCPT1, B0 } \\
\hline INTRCPT2, G00 & 0.427960 & 0.454704 & 0.941 & 29 & 0.354 \\
\hline CHILD_EA, G01 & -0.000004 & 0.000008 & -0.490 & 29 & 0.628 \\
\hline CHILD_WA, G02 & 0.000001 & 0.000010 & 0.127 & 29 & 0.900 \\
\hline INTERNAL, G03 & -0.001577 & 0.005103 & -0.309 & 29 & 0.759 \\
\hline CHILD_SE, G04 & 0.022687 & 0.063703 & 0.356 & 29 & 0.724 \\
\hline CHILD_AG, G05 & 0.004353 & 0.043411 & 0.100 & 29 & 0.921 \\
\hline ETHNIC̄T, G06 & $0.015153 \quad 0$ & 0.015443 & 0.981 & 29 & 0.335 \\
\hline TIME_GAP, G07 & 0.009660 & 0.015230 & 0.634 & 29 & 0.531 \\
\hline \multicolumn{6}{|c|}{ For TIME slope, B1 } \\
\hline INTRCPT2, G10 & 0.005723 & 0.009756 & 0.587 & 107 & 0.559 \\
\hline \multicolumn{6}{|c|}{ For QUAD_TIM slope, B2 } \\
\hline INTRCPT̄2, G20 & 0.013398 & 0.007969 & 1.681 & 36 & 0.101 \\
\hline \multicolumn{6}{|c|}{ For LG_SAA slope, B3 } \\
\hline INTRCPT2, G30 & -0.051100 & 0.111433 & -0.459 & 107 & 0.647 \\
\hline INTERNAL, G31 & 0.051912 & 0.023771 & 2.184 & 107 & 0.03 \\
\hline
\end{tabular}

Final estimation of variance components:

Random Effect Standard Variance df Chi-square P-value Deviation Component

$\begin{array}{lllllll}\text { INTRCPT1, u0 } & 0.15739 & 0.02477 & 29 & 251.27389 & 0.000\end{array}$


QUAD_TIM slope, u2 $\quad 0.02935 \quad 0.00086 \quad 36 \quad 73.54836 \quad 0.000$

$\begin{array}{llll}\text { level-1, } & \mathrm{r} & 0.14033 & 0.01969\end{array}$

Statistics for the current model

Deviance $\quad=-103.400096$

Number of estimated parameters $=16$

Model comparison test

Chi-square statistic $=16.89675$

Degrees of freedom $=6$

P-value $\quad=0.010$

\section{Model 18- Final Model}

Level-1 Model

$$
\text { LG_CORT }=\text { B0 }+ \text { B1 } *(\text { TIME })+\text { B2 } *\left(Q U A D \_T I M\right)+B 3 *\left(L G \_S A A\right)+r
$$

Level-2 Model

$$
\begin{aligned}
& \mathrm{B} 0=\mathrm{G} 00+\mathrm{G} 01 *(\text { INTERNAL })+\mathrm{u} 0 \\
& \mathrm{~B} 1=\mathrm{G} 10 \\
& \mathrm{~B} 2=\mathrm{G} 20+\mathrm{u} 2 \\
& \mathrm{~B} 3=\mathrm{G} 30+\mathrm{G} 31 *(\text { INTERNAL })
\end{aligned}
$$

LG_SAA has been centered around the group mean.

INTERNAL has been centered around the grand mean.

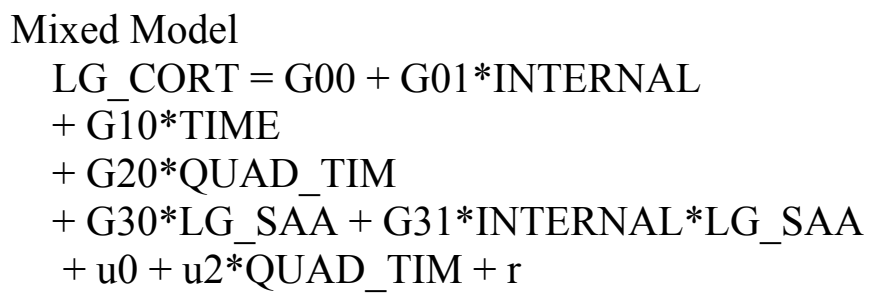

Mixed Model

LG CORT $=\mathrm{G} 00+\mathrm{G} 01 * \mathrm{INTERNAL}$

+ G10*TIME

+ G20*QUAD_TIM

+ G30*LG_SAA + G31*INTERNAL*LG_SAA

$+\mathrm{u} 0+\mathrm{u} 2 * \mathrm{QUAD}$ TIM $+\mathrm{r}$

sigma $^{\wedge} 2=0.01923$

tau

INTRCPT1,B0 $\quad 0.03247 \quad-0.00282$

QUAD_TIM,B2 $-0.00282 \quad 0.00086$ 
tau (as correlations)

INTRCPT1,B0 $1.000-0.534$

QUAD_TIM,B2 -0.534 1.000

Random level-1 coefficient Reliability estimate

INTRCPT1, G0 $\quad 0.881$

QUAD_TIM, G2 $\quad 0.501$

The value of the log-likelihood function at iteration $22=4.080686 \mathrm{E}+001$

The outcome variable is LG_CORT

Final estimation of fixed effects:

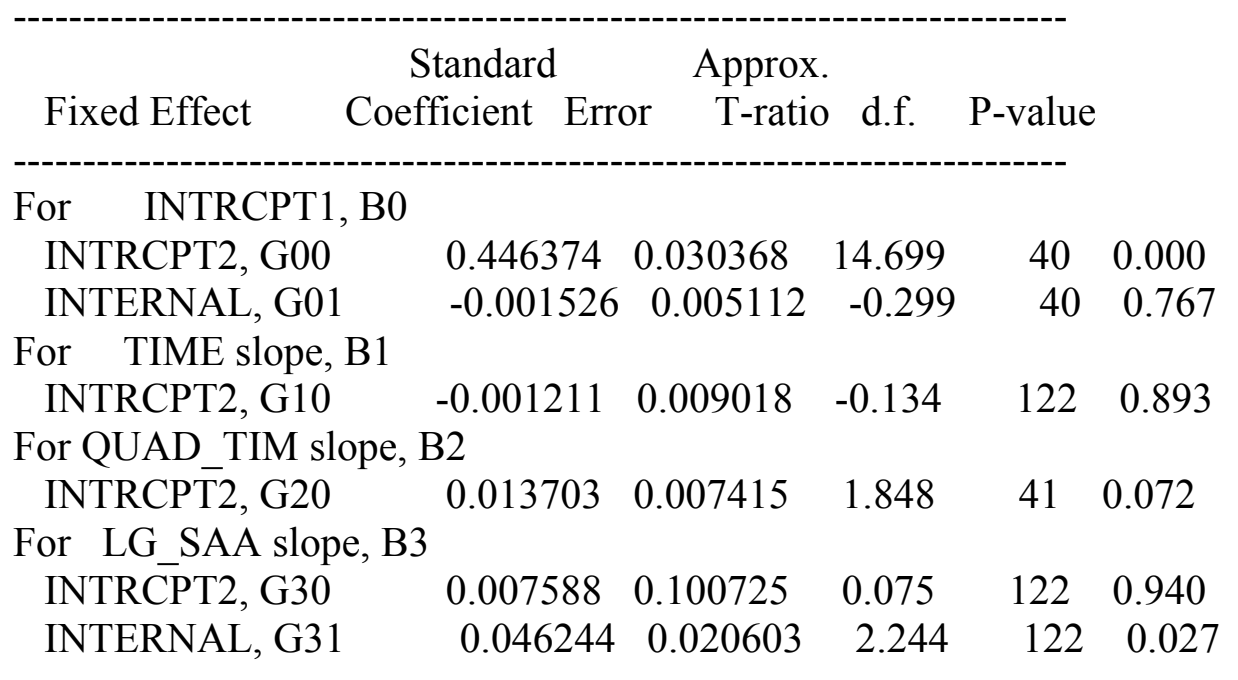

Final estimation of variance components:

Random Effect Standard Variance df Chi-square P-value Deviation Component

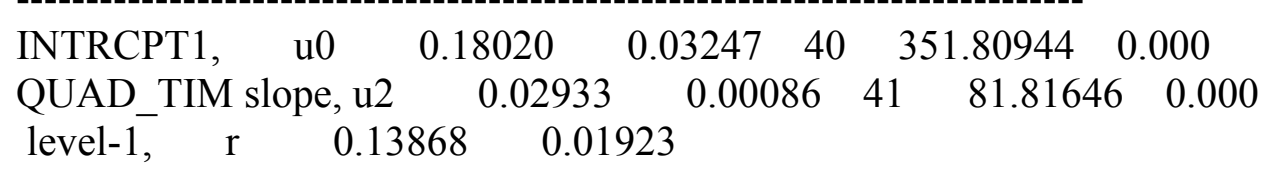

Statistics for current covariance components model

Deviance $\quad=-81.613716$ 
Number of estimated parameters $=4$

Variance-Covariance components test

Chi-square statistic $=38.68313$

Degrees of freedom $=6$

$\mathrm{P}$-value $\quad=\quad<0.001$ 


\section{References}

Adams, R. E., Santo, J. B., \& Bukowski, W. M. (2011). The presence of a best friend buffers the effects of negative experiences. Developmental Psychology, 47, 1786-1791.

Aiken, L. S., \& West, S. G. (1991). Multiple regression: Testing and interpreting interactions. Newbury Park, London, Sage.

Ali, N., \& Pruessner, J. C. (2012). The salivary alpha amylase over cortisol ratio as a marker to assess dysregulations of the stress systems. Physiology \& Behavior, 106, 65-72.

Allwood, M. A., Handwerger, K., Kivlighan, K. T., Granger, D. A., \& Stroud, L. R. (2011). Direct and moderating links of salivary alpha-amylase and cortisol stress-reactivity to youth behavioural and emotional adjustment. Biological Psychology, 88, 57-64.

Achenbach, T. M., \& Rescorla, L. (2001). ASEBA school-age forms \& profiles.

Achenbach, T. M. (2009). Some needed changes in DSM-V: But what about children? Clinical Psychology: Science and Practice, 16, 50-53.

Atkinson, L., Jamieson, B., Khoury, J., Ludmer, J., \& Gonzalez, A. (2016). Stress Physiology in Infancy and Early Childhood: Cortisol Flexibility, Attunement, and Coordination. Journal of Neuroendocrinology, 28, 1-12.

Atkinson, L., Gonzalez, A., Kashy, D. A., Santo Basile, V., Masellis, M., Pereira, J., ... \& Levitan, R. (2013). Maternal sensitivity and infant and mother adrenocortical function across challenges. Psychoneuroendocrinology, 38, 2943-2951.

Angold, A., Costello, E. J., \& Erkanli, A. (1999). Comorbidity. Journal of Child Psychology and Psychiatry, 40, 57-87.

Axelson, D. A., \& Birmaher, B. (2001). Relation between anxiety and depressive disorders in childhood and adolescence. Depression and Anxiety, 14, 67-78. 
Avenevoli, S., Stolar, M., Li, J., Dierker, L., \& Merikangas, K. R. (2001). Comorbidity of depression in children and adolescents: Models and evidence from a prospective high-risk family study. Biological Psychiatry, 49, 1071-1081.

Bae, Y. J., Stadelmann, S., Klein, A. M., Jaeger, S., Hiemisch, A., Kiess, W., ... \& Thiery, J. (2015). The hyporeactivity of salivary cortisol at stress test (TSST-C) in children with internalizing or externalizing disorders is contrastively associated with $\alpha$-amylase. Journal of Psychiatric Research, 71, 78-88.

Bauer, A.M., Quas, J.A., \& Boyce, W.T. (2002). Associations between physiological reactivity and children's behaviour: advantages of a multisystem approach. Journal of Developmental Behavioral Pediatrics, 23, 102-113.

Barber, B. K., \& Olsen, J. A. (2004). Assessing the transitions to middle and high school. Journal of Adolescent Research, 19, 3-30.

Booij, S. H., Bouma, E. M., de Jonge, P., Ormel, J., \& Oldehinkel, A. J. (2013). Chronicity of depressive problems and the cortisol response to psychosocial stress in adolescents: The TRAILS study. Psychoneuroendocrinology, 38, 659-666.

Booth, A., Granger, D. A., \& Shirtcliff, E. A. (2008). Gender-and age-related differences in the association between social relationship quality and trait levels of salivary cortisol. Journal of Research on Adolescence, 18, 239-260.

Blair, C., Granger, D.A., Willoughby, M., \& Kivlighan, K.T. (2006). The Family Life Project Key Investigators. Stress reactivity and regulation in response to emo- tion challenge in 6-month-old infants. Ann NY Acad Sci 2005; 1094: 263-367.

Bonanno, G. A. (2004). Loss, trauma, and human resilience: have we underestimated the human capacity to thrive after extremely aversive events?. American Psychologist, 59, 20-28. 
Bostock, S., Hamer, M., Wawrzyniak, A. J., Mitchell, E. S., \& Steptoe, A. (2011). Positive emotional style and subjective, cardiovascular and cortisol responses to acute laboratory stress. Psychoneuroendocrinology, 36, 1175-1183.

Breland-Noble, A. M., \& Weller, B. (2012). Examining African American adolescent depression in a community sample: The impact of parent/child agreement. Journal of Child and Family Studies, $21,869-876$.

Buske-Kirschbaum, A., Jobst, S., Wustmans, A., Kirschbaum, C., Rauh, W., \& Hellhammer, D. (1997). Attenuated free cortisol response to psychosocial stress in children with atopic dermatitis. Psychosomatic Medicine, 59, 419-426.

Carlson, G. A., Kashani, J. H., Thomas, M. D. F., Vaidya, A., \& Daniel, A. E. (1987). Comparison of two structured interviews on a psychiatrically hospitalized population of children. Journal of the American Academy of Child \& Adolescent Psychiatry, 26, 645-648.

Caspi, A., Bem, D. J., \& Elder, G. H. (1989). Continuities and consequences of interactional styles across the life course. Journal of Personality, 57, 375-406.

Charmandari, E., Kino, T., Souvatzoglou, E., \& Chrousos, G. P. (2003). Pediatric stress: hormonal mediators and human development. Hormone Research in Paediatrics, 59, 161-179.

Chatterton, R. T., Vogelsong, K. M., Lu, Y. C., Ellman, A. B., \& Hudgens, G. A. (1996). Salivary $\alpha$ amylase as a measure of endogenous adrenergic activity. Clinical Physiology, 16, 433-448.

Chavira, D. A., Stein, M. B., Bailey, K., \& Stein, M. T. (2004). Comorbidity of generalized social anxiety disorder and depression in a pediatric primary care sample. Journal of Affective Disorders, 80, 163-171. 
Chen, F. R., Raine, A., Soyfer, L., \& Granger, D. A. (2015). Interaction of adrenocortical activity and autonomic arousal on children's externalizing and internalizing behavior problems. Journal of Abnormal Child Psychology, 43, 189-202.

Cicchetti, D., \& Toth, S. L. (1991). A developmental perspective on internalizing and externalizing disorders. Internalizing and Externalizing Expressions of Dysfunction, 2, 1-19.

Chopra, K.K., Ravindran, A., Kennedy, S.H., Mackenzie, B., Mat- thews, S., Anisman, H., et al., 2009. Sex differences in hormonal responses to a social stressor in chronic major depression. Psychoneuroendocrinology 34, 1235-1241.

Chrousos, G. P. (2009). Stress and disorders of the stress system. Nature Reviews Endocrinology, 5, 374-381.

Colich, N. L., Kircanski, K., Foland-Ross, L. C., \& Gotlib, I. H. (2015). HPA-axis reactivity interacts with stage of pubertal development to predict the onset of depression. Psychoneuroendocrinology, 55, $94-101$.

Collins, W. A. (Ed.). (1984). Development during middle childhood: The years from six to twelve. Washington, DC: National Academy Press.

Costello, E. J., Mustillo, S., Erkanli, A., Keeler, G., \& Angold, A. (2003). Prevalence and development of psychiatric disorders in childhood and adolescence. Archives of General Psychiatry, 60, 837844.

Danese, A., \& McEwen, B. S. (2012). Adverse childhood experiences, allostasis, allostatic load, and age-related disease. Physiology \& Behavior, 106, 29-39.

Dawson, J. F. (2014). Moderation in management research: What, why, when and how. Journal of Business and Psychology, 29, 1-19.

de Carvalho Tofoli, S. M., Von Werne Baes, C., Martins, C. M. S., \& Juruena, M. (2011). Early life 
stress, HPA axis, and depression. Psychology \& Neuroscience, 4, 229.

De Los Reyes, A., \& Kazdin, A. E. (2005). Informant discrepancies in the assessment of childhood psychopathology: a critical review, theoretical framework, and recommendations for further study. Psychological Bulletin, 131, 483.

Del Giudice, M., Ellis, B. J., \& Shirtcliff, E. A. (2011). The Adaptive Calibration Model of stress responsivity. Neuroscience and Biobehavioral Reviews, 35, 1562-1592.

De Los Reyes, A., \& Kazdin, A. E. (2005). Informant discrepancies in the assessment of childhood psychopathology: a critical review, theoretical framework, and recommendations for further study. Psychological Bulletin, 131, 483.

de Veld, D. M., Riksen-Walraven, J. M., \& de Weerth, C. (2012). The relation between emotion regulation strategies and physiological stress responses in middle childhood. Psychoneuroendocrinology, 37, 1309-1319.

de Veld, D. M., Riksen-Walraven, J. M., \& de Weerth, C. (2014). Does the arrival index predict physiological stress reactivity in children. Stress, 17, 383-388.

Dickerson, S. S., \& Kemeny, M. E. (2004). Acute stressors and cortisol responses: a theoretical integration and synthesis of laboratory research. Psychological Bulletin, 130, 355-391.

Doom, J. R., Doyle, C. M., \& Gunnar, M. R. (2017). Social stress buffering by friends in childhood and adolescence: Effects on HPA and oxytocin activity. Social Neuroscience, 12, 8-21.

Doom, J. R., Doyle, C. M., \& Gunnar, M. R. (2017). Social stress buffering by friends in childhood and adolescence: Effects on HPA and oxytocin activity. Social Neuroscience, 12, 8-21.

Dorn, L. D., \& Rotenstein, D. (2004). Early puberty in girls: The case of premature adrenarche. Women's Health Issues, 14, 177-183.

El-Sheikh, M., Erath, S. A., Buckhalt, J. A., Granger, D. A., \& Mize, J. (2008). Cortisol and children's 
adjustment: The moderating role of sympathetic nervous system activity. Journal of Abnormal Child Psychology, 36, 601-611.

Essau, C. A. (2008). Comorbidity of depressive disorders among adoles- cents in community and clinical settings. Psychiatry Research, 158, 35-42.

Fekedulegn, D. B., Andrew, M. E., Burchfiel, C. M., Violanti, J. M., Hartley, T. A., Charles, L. E., \& Miller, D. B. (2007). Area under the curve and other summary indicators of repeated waking cortisol measurements. Psychosomatic Medicine, 69, 651-659.

Flinn, M. V. (2006). Evolution and ontogeny of stress response to social challenges in the human child. Developmental Review, 26, 138-174.

Foland-Ross, L. C., Hamilton, J. P., Joormann, J., Berman, M. G., Jonides, J., \& Gotlib, I. H. (2013). The neural basis of difficulties disengaging from negative irrelevant material in major depression. Psychological Science, 24, 334-344.

Fortunato, C. K., Dribin, A. E., Granger, D. A., \& Buss, K. A. (2008). Salivary alpha-amylase and cortisol in toddlers: Differential relations to affective behavior. Developmental Psychobiology, $50,807-818$.

Frankenhaeuser, M., Lundberg, U., \& Forsman, L. (1980). Dissociation between sympathetic-adrenal and pituitary-adrenal responses to an achievement situation characterized by high controllability: comparison between type A and type B males and females. Biological Psychology, 10, 79-91.

Fries, E., Hesse, J., Hellhammer, J., \& Hellhammer, D. H. (2005). A new view on hypocortisolism. Psychoneuroendocrinology, 30, 1010-1016. 
Gallagher, P., Reid, K. S., \& Ferrier, I. N. (2009). Neuropsychological functioning in health and mood disorder: modulation by glucocorticoids and their receptors. Psychoneuroendocrinology, 34, S196-S207.

Ganzel, B.L., Morris, P.A., Wethington, E., 2010. Allostasis and the human brain: inte- grating models of stress from the social and life sciences. Psychology Review, 117, 134-174.

Giles, G. E., Mahoney, C. R., Brunyé, T. T., Taylor, H. A., \& Kanarek, R. B. (2014). Stress effects on mood, HPA axis, and autonomic response: comparison of three psychosocial stress paradigms. PloS one, 9, e113618.

Gold, P. W., \& Chrousos, G. P. (2002). Organization of the stress system and its dysregulation in melancholic and atypical depression: high vs low CRH/NE states. Molecular Psychiatry, 7, 254-275.

Goldberg, S., Levitan, R., Leung, E., Masellis, M., Basile, V., Nemeroff, C .B, \& Atkinson L., (2003). Stability of baseline and stress cortisol concentrations in 12-18-month-old infants: Issues of time, location, and stressor. Biological Psychiatry, 54, 719-726.

Goldstein, D. S., \& Kopin, I. J. (2008). Adrenomedullary, adrenocortical, and sympathoneural responses to stressors: a meta-analysis. Endocrine Regulation, 42, 111-119.

Goldstein, D. S., \& McEwen, B. (2002). Allostasis, homeostats, and the nature of stress. Stress: The International Journal on the Biology of Stress, 5, 55-58.

Gordis, E. B., Granger, D. A., Susman, E. J., \& Trickett, P. K. (2008). Salivary alpha amylase-cortisol asymmetry in maltreated youth. Hormones and Behavior, 53, 96-103.

Graber, J. A., \& Sontag, L. M. (2009). Handbook of adolescent psychology.

Garber, J., \& Weersing, V. R. (2010). Comorbidity of anxiety and depression in youth: implications for treatment and prevention. Clinical Psychology: Science and Practice, 17(4), 293-306. 
Granger, D. A., Kivlighan, K. T., Blair, C., El-Sheikh, M., Mize, J., Lisonbee, J. A., ... \& Schwartz, E. B. (2006). Integrating the measurement of salivary $\alpha$-amylase into studies of child health, development, and social relationships. Journal of Social and Personal Relationships, 23, $267-$ 290.

Granger, D. A., Kivlighan, K. T., El-Sheikh, M., Gordis, E., \& Stroud, L. (2008). Assessment of salivary alpha-amylase in biobehavioral research. Handbook of physiological research methods in health psychology. Thousand Oaks, CA: Sage Publications, 95-113.

Granger, D. A., Weisz, J. R., \& Kauneckis, D. (1994). Neuroendocrine reactivity, internalizing behavior problems, and control-related cognitions in clinic-referred children and adolescents. Journal of abnormal psychology, 103(2), 267-276.

Guilliams, T. G., \& Edwards, L. (2010). Chronic stress and the HPA axis. The Standard, 9(2), 1-12. Gunnar, M., \& Quevedo, K. (2007). The neurobiology of stress and development. Annual Review of Psychopathology, 58, 145-173.

Gunnar, M. R., Talge, N. M., \& Herrera, A. (2009). Stressor paradigms in developmental studies: What does and does not work to produce mean increases in salivary cortisol. Psychoneuroendocrinology, 34, 953-967.

Gunnar, M. R., \& Vazquez, D. (2006). Stress neurobiology and developmental psychopathology. In D. Cicchetti \& D. J. Cohen (Eds.), Developmental psychopathology: Developmental neuroscience (pp. 533-577). Hoboken, NJ, US: John Wiley \& Sons Inc.

Gunnar, M. R., Brodersen, L., Krueger, K., \& Rigatuso, J. (1996). Dampening of adrenocortical responses during infancy: Normative changes and individual differences. Child Development, $67,877-889$ 
Gustafsson, P. E., Anckarsäter, H., Lichtenstein, P., Nelson, N., \& Gustafsson, P. A. (2010). Does quantity have a quality all its own? Cumulative adversity and up-and down-regulation of circadian salivary cortisol levels in healthy children. Psychoneuroendocrinology, 35, 14101415.

Habib, K. E., Weld, K. P., Rice, K. C., Pushkas, J., Champoux, M., Listwak, S., ... \& Chrousos, G. P. (2000). Oral administration of a corticotropin-releasing hormone receptor antagonist significantly attenuates behavioral, neuroendocrine, and autonomic responses to stress in primates. Proceedings of the National Academy of Sciences, 97, 6079-6084.

Haggerty, R. J., Garmezy, N., Sherrod, L. R., \& Rutter, M. (1994). Stress, risk, and resilience in children and adolescents: Processes, mechanisms, and interventions (pp. 64-99). Cambridge: Cambridge University Press.

Hamer, M., Williams, E., Vuonovirta, R., Giacobazzi, P., Gibson, E. L., \& Steptoe, A. (2006). The effects of effort-reward imbalance on inflammatory and cardiovascular responses to mental stress. Psychosomatic Medicine, 68, 408-413.

Hammen, C. (2005). Stress and depression. Annu. Rev. Clin. Psychol., 1, 293-319.

Hankin, B. L., Badanes, L. S., Abela, J. R., \& Watamura, S. E. (2010). Hypothalamic-pituitary-adrenal axis dysregulation in dysphoric children and adolescents: Cortisol reactivity to psychosocial stress from preschool through middle adolescence. Biological Psychiatry, 68, 484-490.

Harkness, K. L., Stewart, J. G., \& Wynne-Edwards, K. E. (2011). Cortisol reactivity to social stress in adolescents: role of depression severity and child maltreatment. Psychoneuroendocrinology, 36, $173-181$.

Hartman, C.A., Hermanns, V. W., de Jong, P. J., \& Ormel, J. (2013). Self- or parent report of (cooccurring) internalizing and externalizing problems, and basal or reactivity measures of HPA- 
axis functioning: A systematic evaluation of the internalizing-hyperresponsivity versus externalizing-hyporesponsivity HPA-axis hypothesis. Biological Psychology, 94, 175-184.

Heim, C., Ehlert, U., \& Hellhammer, D. H. (2000). The potential role of hypocortisolism in the pathophysiology of stress-related bodily disorders. Psychoneuroendocrinology, 25, 1-35.

Herman, J. P., Figueiredo, H., Mueller, N. K., Ulrich-Lai, Y., Ostrander, M. M., Choi, D. C., \& Cullinan, W. E. (2003). Central mechanisms of stress integration: hierarchical circuitry controlling hypothalamo-pituitary-adrenocortical responsiveness. Frontiers in Neuroendocrinology, 24, 151-180.

Hibel, L. C., Granger, D. A., Blair, C., \& Cox, M. J. (2009). Intimate partner violence moderates the association between mother-infant adrenocortical activity across an emotional challenge. Journal of Family Psychology, 23, 615-625.

Hill-Soderlund, A. L., Holochwost, S. J., Willoughby, M. T., Granger, D. A., Gariépy, J. L., MillsKoonce, W. R., \& Cox, M. J. (2015). The developmental course of salivary alpha-amylase and cortisol from 12 to 36 months: Relations with early poverty and later behavior problems. Psychoneuroendocrinology, 52, 311-323.

Hostinar, C. E., \& Gunnar, M. R. (2013). Future directions in the study of social relationships as regulators of the HPA axis across development. Journal of Clinical Child \& Adolescent Psychology, 42, 564-575.

Hruschka, D.J., Kohrt, B.A., Worthman, C.M., 2005. Estimating between- and within-individual variation in cortisol levels using multilevel models. Psychoneuroendocrinology 30, 698-714.

Jaffee, S. R., McFarquhar, T., Stevens, S., Ouellet-Morin, I., Melhuish, E., \& Belsky, J. (2015). Interactive effects of early and recent exposure to stressful contexts on cortisol reactivity in middle childhood. Journal of Child Psychology and Psychiatry, 56, 138-146. 
Jansen, J., Beijers, R., Riksen-Walraven, M., \& de Weerth, C. (2010). Cortisol reactivity in young infants. Psychoneuroendocrinology, 35, 329-338.

Janssens, K. A., Oldehinkel, A. J., Verhulst, F. C., Hunfeld, J. A., Ormel, J., \& Rosmalen, J. G. (2012). Symptom-specific associations between low cortisol responses and functional somatic symptoms: the TRAILS study. Psychoneuroendocrinology, 37(3), 332-340.

Kaltsas, G. A., \& Chrousos, G. P. (2007). The neuroendocrinology of stress. Handbook of Psychophysiology, 3, 303-318.

Katz, J.L., Weiner, H., Gallagher, T.F., \& Hellman, L. (1970). Stress, distress, and ego defenses. Psychoendocrine response to impending breast tumor biopsy. Archives of General Psychiatry, $23,131-142$.

Kazdin, A. E. (1994). Methodology, design, and evaluation in psychotherapy research.

Keenan, K., Hipwell, A., Babinski, D., Bortner, J., Henneberger, A., Hinze, A., ... \& Sapotichne, B. (2013). Examining the developmental interface of cortisol and depression symptoms in young adolescent girls. Psychoneuroendocrinology, 38, 2291-2299.

Kendler, K. S., Kuhn, J., \& Prescott, C. A. (2004). The interrelationship of neuroticism, sex, and stressful life events in the prediction of episodes of major depression. American Journal of Psychiatry, 161, 631-636.

Khoury, J. E., Gonzalez, A., Levitan, R., Goodwill, A., Masellis, M., Vincenzo, B., \& Atkinson, L. (2015). Summary cortisol reactivity indicators: interrelations and meaning. Neurobiology of Stress, 2, 34-43.

Kirschbaum, C., Pirke, K. M., \& Hellhammer, D. H. (1993). The 'Trier Social Stress Test'-a tool for investigating psychobiological stress responses in a laboratory setting. Neuropsychobiology, 28, 76-81. 
Kirschbaum, C., \& Hellhammer, D. H. (1994). Salivary cortisol in psychoneuroendocrine research: recent developments and applications. Psychoneuroendocrinology, 19, 313-333.

Koss, K. J., George, M. R., Cummings, E. M., Davies, P. T., El-Sheikh, M., \& Cicchetti, D. (2014). Asymmetry in children's salivary cortisol and alpha-amylase in the context of marital conflict: Links to children's emotional security and adjustment. Developmental Psychobiology, 56, 836849.

Kovacs, M. (1992). Children's depression inventory: Manual. Multi-Health Systems.

Krämer, M., Seefeldt, W. L., Heinrichs, N., Tuschen-Caffier, B., Schmitz, J., Wolf, O. T., \& Blechert, J. (2012). Subjective, autonomic, and endocrine reactivity during social stress in children with social phobia. Journal of Abnormal Child Psychology, 40, 95-104.

Kudielka, B. M., \& Kirschbaum, C. (2005). Sex differences in HPA axis responses to stress: a review. Biological Psychology, 69, 113-132.

Laurent, H. K., Ablow, J. C., \& Measelle, J. (2012). Taking stress response out of the box: Stability, discontinuity, and temperament effects on HPA and SNS across social stressors in motherinfant dyads. Developmental Psychology, 48, 35.

Laurent, H. K., Powers, S. I., \& Granger, D. A. (2013). Refining the multisystem view of the stress response: Coordination among cortisol, alpha-amylase, and subjective stress in response to relationship conflict. Physiology \& Behavior, 119, 52-60.

Laurent, H. K., Gilliam, K. S., Wright, D. B., \& Fisher, P. A. (2015). Child anxiety symptoms related to longitudinal cortisol trajectories and acute stress responses: evidence of developmental stress sensitization. Journal of Abnormal Psychology, 124, 68-79. 
Laurent, H., Vergara-Lopez, C., \& Stroud, L. R. (2016). Differential relations between youth internalizing/externalizing problems and cortisol responses to performance vs. interpersonal stress. Stress, 19, 492-498.

Lesage, J., Sebaai, N., Leonhardt, M., Dutriez-Casteloot, I., Breton, C., Deloof, S., \& Vieau, D. (2006). Perinatal maternal undernutrition programs the offspring hypothalamo-pituitary-adrenal (HPA) axis. Stress: The International Journal on the Biology of Stress, 9, 183-198.

Levine, S. (2005). Developmental determinants of sensitivity and resistance to stress. Psychoneuroendocrinology, 30, 939-946.

LeMoult, J., Chen, M. C., Foland-Ross, L. C., Burley, H. W., \& Gotlib, I. H. (2015). Concordance of mother-daughter diurnal cortisol production: Understanding the intergenerational transmission of risk for depression. Biological Psychology, 108, 98-104.

Leppert, K. A., Kushner, M., Smith, V. C., Lemay, E. P., \& Dougherty, L. R. (2016). Children's cortisol responses to a social evaluative laboratory stressor from early to middle childhood. Developmental Psychobiology, 58, 1019-1033.

Little, Todd D. Longitudinal structural equation modeling. Guilford Press, 2013.

Lopez, B., Turner, R. J., \& Saavedra, L. M. (2005). Anxiety and risk for substance dependence among late adolescents/young adults. Journal of Anxiety Disorders, 19, 275-294.

Lopez-Duran, N. L., Hajal, N. J., Olson, S. L., Felt, B. T., \& Vazquez, D. M. (2009). Individual differences in cortisol responses to fear and frustration during middle childhood. Journal of experimental child psychology, 103(3), 285-295. 
Ludmer, J. A., Levitan, R., Gonzalez, A., Kennedy, J., Villani, V., Masellis, M., ... \& Atkinson, L. (2015). DRD2 and SLC6A3 moderate impact of maternal depressive symptoms on infant cortisol. Psychoneuroendocrinology, 62, 243-251.

Lundberg, U., \& Frankenhaeuser, M. (1980). Pituitary-adrenal and sympathetic-adrenal correlates of distress and effort. Journal of Psychosomatic Research, 24, 125-130.

Maruyama, Y., Kawano, A., Okamoto, S., Ando, T., Ishitobi, Y., Tanaka, Y., ... \& Ninomiya, T. (2012). Differences in salivary alpha-amylase and cortisol responsiveness following exposure to electrical stimulation versus the Trier Social Stress Tests. PLoS One, 7, e39375.

Masip, A. F., Amador-Campos, J. A., Gómez-Benito, J., \& del Barrio Gándara, V. (2010). Psychometric properties of the Children's Depression Inventory in community and clinical sample. The Spanish Journal of Psychology, 13, 990-999.

McEwen, B. S. (2007). Physiology and neurobiology of stress and adaptation: central role of the brain. Physiological Reviews, 87, 873-904.

McEwen, B. S. (1998). Protective and damaging effects of stress mediators. New England Journal of Medicine, 338, 171-179.

McEwen, B. S., \& Seeman, T. (1999). Protective and damaging effects of mediators of stress: elaborating and testing the concepts of allostasis and allostatic load. Annals of the New York Academy of Sciences, 896, 30-47.

Meaney, M. J., \& Szyf, M. (2005). Environmental programming of stress responses through DNA methylation: life at the interface between a dynamic environment and a fixed genome. Dialogues in Clinical Neuroscience, 7, 103-123.

Miller, G. E., Chen, E., \& Zhou, E. S. (2007). If it goes up, must it come down? Chronic stress and the hypothalamic-pituitary-adrenocortical axis in humans. Psychological Bulletin, 133, 25-45. 
Monroe, S. M., Rohde, P., Seeley, J. R., \& Lewinsohn, P. M. (1999). Life events and depression in adolescence: relationship loss as a prospective risk factor for first onset of major depressive disorder. Journal of Abnormal Psychology, 108, 606-614.

Morrison, W. E., Haas, E. C., Shaffner, D. H., Garrett, E. S., \& Fackler, J. C. (2003). Noise, stress, and annoyance in a pediatric intensive care unit. Critical Care Medicine, 31, 113-119.

Nachmias, M., Gunnar, M., Mangelsdorf, S., Parritz, R. H., \& Buss, K. (1996). Behavioral inhibition and stress reactivity: The moderating role of attachment security. Child Development, 508-522.

Nater, U. M., La Marca, R., Florin, L., Moses, A., Langhans, W., Koller, M. M., \& Ehlert, U. (2006). Stress-induced changes in human salivary alpha-amylase activity_associations with adrenergic activity. Psychoneuroendocrinology, 31, 49-58.

Nater, U. M., \& Rohleder, N. (2009). Salivary alpha-amylase as a non-invasive biomarker for the sympathetic nervous system: current state of research. Psychoneuroendocrinology, 34, 486-496.

Nater, U. M., Rohleder, N., Gaab, J., Berger, S., Jud, A., Kirschbaum, C., \& Ehlert, U. (2005). Human salivary alpha-amylase reactivity in a psychosocial stress paradigm. International Journal of Psychophysiology, 55, 333-342.

Nigg, J. T. (2006). Temperament and developmental psychopathology. Journal of Child Psychology and Psychiatry, 47, 395-422.

O’Keane, V., Frodl, T., \& Dinan, T. G. (2012). A review of Atypical depression in relation to the course of depression and changes in HPA axis organization. Psychoneuroendocrinology, 37, 1589-1599.

O’Sullivan, C. (2004). The psychosocial determinants of depression: A lifespan perspective. Journal of Nervous and Mental Disease, 192, 585-594. 
Patton, G. C., Olsson, C., Bond, L., Toumbourou, J. W., Carlin, J. B., Hemphill, S. A., \& Catalano, R. F. (2008). Predicting female depression across puberty: a two-nation longitudinal study. Journal of the American Academy of Child \& Adolescent Psychiatry, 47, 1424-1432.

Peters, M. L., Godaert, G. L., Ballieux, R. E., van Vliet, M., Willemsen, J. J., Sweep, F. C., \& Heijnen, C. J. (1998). Cardiovascular and endocrine responses to experimental stress: effects of mental effort and controllability. Psychoneuroendocrinology, 23, 1-17.

Pruessner, J. C., Wolf, O. T., Hellhammer, D. H., Buske-Kirschbaum, A., Von Auer, K., Jobst, S., ... \& Kirschbaum, C. (1997). Free cortisol levels after awakening: a reliable biological marker for the assessment of adrenocortical activity. Life Sciences, 61, 2539-2549.

Rainey, W. E., Carr, B. R., Sasano, H., Suzuki, T., \& Mason, J. I. (2002). Dissecting human adrenal androgen production. Trends in Endocrinology and Metabolism, 13, 234-239.

Raudenbush, S. W., Brennan, R. T., \& Barnett, R. C. (1995). A multivariate hierarchical model for studying psychological change within married couples. Journal of Family Psychology, 9, 161.

Raudenbush, S. W., \& Bryk, A. S. (2002). Hierarchical linear models: Applications and data analysis methods (Vol. 1). Sage.

Raudenbush, S. W., Bryk, A. S., Cheong, Y. F., Congdon, R., \& Du Toit, M. (2011). Hierarchical linear and nonlinear modeling (HLM7). Lincolnwood, IL: Scientific Software International.

Reynolds, R. M. (2013). Glucocorticoid excess and the developmental origins of disease: two decades of testing the hypothesis-2012 Curt Richter Award Winner. Psychoneuroendocrinology, 38, 111.

Reeves, J. W., Fisher, A. J., Newman, M. G., \& Granger, D. A. (2016). Sympathetic and hypothalamicpituitary-adrenal asymmetry in generalized anxiety disorder. Psychophysiology, 53, 951-957. 
Rohleder, N., Nater, U. M., Wolf, J. M., Ehlert, U., \& Kirschbaum, C. (2004). Psychosocial stressinduced activation of salivary alpha-amylase: an indicator of sympathetic activity?. Annals of the New York Academy of Sciences, 1032, 258-263.

Ruttle, P. L., Javaras, K. N., Klein, M. H., Armstrong, J. M., Burk, L. R., \& Essex, M. J. (2013). Concurrent and longitudinal associations between diurnal cortisol and body mass index across adolescence. Journal of Adolescent Health, 52, 731-737.

Sameroff, A. J., \& Haith, M. M. (Eds.). (1996). The five to seven year shift: The age of reason and responsibility. Chicago: University of Chicago Press.

Sapolsky, R. M. (2000). Glucocorticoids and hippocampal atrophy in neuropsychiatric disorders. Archives of General Psychiatry, 57, 925-935.

Sapolsky, R. M., Romero, L. M., \& Munck, A. U. (2000). How do glucocorticoids influence stress responses? Integrating permissive, suppressive, stimulatory, and preparative actions 1. Endocrine Reviews, 21, 55-89.

Schumacher, S., Kirschbaum, C., Fydrich, T., \& Ströhle, A. (2013). Is salivary alpha-amylase an indicator of autonomic nervous system dysregulations in mental disorders?-A review of preliminary findings and the interactions with cortisol. Psychoneuroendocrinology, 38, 729743.

Seltzer, L. J., Ziegler, T. E., \& Pollak, S. D. (2010). Social vocali- zations can release oxytocin in humans. Proceedings of the Royal Society B: Biological Sciences, 277, 2661-2666.

Singer, J. D., \& Willet, J. B. (2003). A framework for investigating change over time. Applied longitudinal data analysis: Modeling change and event occurrence, 3-15. 
Skoluda, N., Strahler, J., Schlotz, W., Niederberger, L., Marques, S., Fischer, S., ... \& Nater, U. M. (2015). Intra-individual psychological and physiological responses to acute laboratory stressors of different intensity. Psychoneuroendocrinology, 51, 227-236.

Snijders, T. B. (2005). Power and Sample Size in Multilevel Linear Models. In B.S. Everitt and D.C. Howell (Eds.), Encyclopedia of Statistics in Behavioral Science. Volume 3, 1570-1573. Wiley.

Spies, L. A., Margolin, G., Susman, E. J., \& Gordis, E. B. (2011). Adolescents' cortisol reactivity and subjective distress in response to family conflict: The moderating role of internalizing symptoms. Journal of Adolescent Health, 49, 386-392.

Spinrad, T. L., Eisenberg, N., Granger, D. A., Eggum, N. D., Sallquist, J., Haugen, R. G., ... \& Hofer, C. (2009). Individual differences in preschoolers' salivary cortisol and alpha-amylase reactivity: Relations to temperament and maladjustment. Hormones and Behavior, 56, 133-139.

Stalder, T., \& Kirschbaum, C. (2012). Analysis of cortisol in hair-State of the art and future directions. Brain, Behavior, and Immunity, 26, 1019-1029.

Steeger, C. M., Cook, E. C., \& Connell, C. M. (2016). The interactive effects of stressful family life events and cortisol reactivity on adolescent externalizing and internalizing behaviors. Child Psychiatry \& Human Development, 1-10.

Sterling, P., \& Eyer, J. (1988). Allostasis: a new paradigm to explain arousal pathology. In: Fisher SRJ, editor. Handbook of life stress, cognition and health. New York: John Wiley.

Stratakis, C. A., \& Chrousos, G. P. (1995). Neuroendocrinology and pathophysiology of the stress system. Annals of the New York Academy of Sciences, 771, 1-18.

Stroop, J. R. (1935). Studies of interference in serial verbal reactions. Journal of Experimental Psychology, 18, 643.

Stroud, L. R., Foster, E., Papandonatos, G. D., Handwerger, K., Granger, D. A., Kivlighan, K. T., \& 
Niaura, R. (2009). Stress response and the adolescent transition: Performance versus peer rejection stressors. Development and Psychopathology, 21, 47-68.

Stroud, L. R., Papandonatos, G. D., Williamson, D. E., \& Dahl, R. E. (2011). Sex differences in cortisol response to corticotropin releasing hormone challenge over puberty: Pittsburgh Pediatric Neurobehavioral Studies. Psychoneuroendocrinology, 36, 1226-1238.

Suzuki, H., Belden, A. C., Spitznagel, E., Dietrich, R., \& Luby, J. L. (2013). Blunted stress cortisol reactivity and failure to acclimate to familiar stress in depressed and sub-syndromal children. Psychiatry research, 210, 575-583.

Tarullo, A. R., \& Gunnar, M. R. (2006). Child maltreatment and the developing HPA axis. Hormones and behavior, 50, 632-639.

Tilbrook, A. J., Turner, A. I., \& Clarke, I. J. (2002). Stress and reproduction: Central mechanisms and sex differences in non-rodent species. Stress, 5, 83-100.

Tops, M., Riese, H., Oldehinkel, A. J., Rijsdijk, F. V., \& Ormel, J. (2008). Rejection sensitivity relates to hypocortisolism and depressed mood state in young women. Psychoneuroendocrinology, 33, $551-559$.

Twenge, J. M., \& Nolen-Hoeksema, S. (2002). Age, gender, race, socioeconomic status, and birth cohort difference on the children's depression inventory: A meta-analysis. Journal of Abnormal Psychology, 111, 578-588.

Turner-Cobb, J. (2005). Psychological and stress hormone correlates in early life: A key to HPA-axis dysregulation and normalisation. Stress: The International Journal on the Biology of Stress, 8 , 47-57.

Ulrich-Lai, Y. M., \& Herman, J. P. (2009). Neural regulation of endocrine and autonomic stress responses. Nature Reviews Neuroscience, 10, 397-409. 
Ulrike, S., Reinhold, L., \& Dirk, H. (2013). Major depression in young girls is related to altered cortisol awakening response. European Child \& Adolescent Psychiatry, 22, 379.

Valentino et al., 1998

Van den Bergh, B. R., \& Van Calster, B. (2009). Diurnal cortisol profiles and evening cortisol in postpubertal adolescents scoring high on the Children's Depression Inventory.

Psychoneuroendocrinology, 34, 791-794.

van Stegeren, A. H., Wolf, O. T., \& Kindt, M. (2008). Salivary alpha amylase and cortisol responses to different stress tasks: impact of sex. International Journal of Psychophysiology, 69, 33-40.

van der Voorn, B., Hollanders, J. J., Ket, J. C., Rotteveel, J., \& Finken, M. J. (2017). Gender-specific differences in hypothalamus-pituitary-adrenal axis activity during childhood: a systematic review and meta-analysis. Biology of Sex Differences, 8, 3.

Viau, V. (2002). Functional cross-talk between the hypothalamic-pituitary-gonadal-adrenal axes. Journal of Neuroendocrinology, 14, 506-513.

Viau, V., Bingham, B., Davis, J., Lee, P., \& Wong, M. (2005). Gender and puberty interact on the stress-induced activation of parvocellular neurosecretory neurons and corticotropin-releasing hormone messenger ribonucleic acid expression in the rat. Endocrinology, 146, 137-146.

Vigil, J. M., Geary, D. C., Granger, D. A., \& Flinn, M. V. (2010). Sex differences in salivary cortisol, alpha-amylase, and psychological functioning following Hurricane Katrina. Child Development, $81,1228-1240$.

Willett, J. B., \& Sayer, A. G. (1994). Using covariance structure analysis to detect correlates and predictors of individual change over time. Psychological Bulletin, 116, 363.

Williamson, D. E., Birmaher, B., Dahl, R. E., \& Ryan, N. D. (2005). Stressful life events in anxious and depressed children. Journal of Child \& Adolescent Psychopharmacology, 15, 571-580. 
Wolff, C.T., Hofer, M., \& Mason, J.W. (1964). Relationship between psychological defenses and mean urinary 17-hydroxicorticosteroid excretion rates. Methodologic and theoretical considerations. Psychosomatic Medicine, 26, 592-609.

Yim, I. S., Quas, J. A., Cahill, L., \& Hayakawa, C. M. (2010). Children's and adults' salivary cortisol responses to an identical psychosocial laboratory stressor. Psychoneuroendocrinology, 35, 241248.

Yorbik, O., Birmaher, B., Axelson, D., Williamson, D. E., \& Ryan, N. D. (2004). Clinical characteristics of depressive symptoms in children and adolescents with major depressive disorder. Journal of Clinical Psychiatry, 65, 1654-1659.

Young, E. A., Lopez, J. F., Murphy-Weinberg, V., Watson, S. J., \& Akil, H. (2000). Hormonal evidence for altered responsiveness to social stress in major depression. Neuropsychopharmacology, 23, 411-418.

Zahn-Waxler, C., Klimes-Dougan, B., \& Slattery, M. J. (2000). Internalizing problems of childhood and adolescence: Prospects, pitfalls, and progress in understanding the development of anxiety and depression. Development and Psychopathology, 12, 443-466.

Zilioli, S., \& Watson, N. V. (2012). The hidden dimensions of the competition effect: Basal cortisol and basal testosterone jointly predict changes in salivary testosterone after social victory in men. Psychoneuroendocrinology, 37, 1855-1865. 